\title{
RESULTS OF TOXICITY TESTS ON SRS NPDES OUTFALLS A-01, A-11 AND G-10, JANUARY - SEPTEMBER 2002
}

\author{
by \\ WINONA L. SPECHT \\ SAVANNAH RIVER TECHNOLOGY CENTER
}

Approved by:

John B. Gladden, Section Manager

Environmental Analysis Section

Savannah River Technology Center

Publication Date: $\quad$ October, 2002

WESTINGHOUSE SAVANNAH RIVER COMPANY

SAVANNAH RIVER SITE

AIKEN, SC 29808

THIS DOCUMENT WAS PREPARED IN CONNECTION WITH WORK UNDER U.S. DEPARTMENT OF ENERGY CONTRACT DE-AC09-96SR18500. By acceptance of this paper, the publisher and/or recipient acknowledges the U.S. Government's right to retain a nonexclusive, royalty-free license in and to any copyright covering this paper, along with the right to reproduce and to authorize others to reproduce all or any part of the copyrighted paper. 
This document was prepared in conjunction with work accomplished under Contract No. DE-AC09-96SR18500 with the U. S. Department of Energy.

\section{DISCLAIMER}

This report was prepared as an account of work sponsored by an agency of the United States Government. Neither the United States Government nor any agency thereof, nor any of their employees, makes any warranty, express or implied, or assumes any legal liability or responsibility for the accuracy, completeness, or usefulness of any information, apparatus, product or process disclosed, or represents that its use would not infringe privately owned rights. Reference herein to any specific commercial product, process or service by trade name, trademark, manufacturer, or otherwise does not necessarily constitute or imply its endorsement, recommendation, or favoring by the United States Government or any agency thereof. The views and opinions of authors expressed herein do not necessarily state or reflect those of the United States Government or any agency thereof.

This report has been reproduced directly from the best available copy.

Available for sale to the public, in paper, from: U.S. Department of Commerce, National Technical Information Service, 5285 Port Royal Road, Springfield, VA 22161, phone: (800) 553-6847, fax: (703) 605-6900

email: orders@ntis.fedworld.gov

online ordering: http://www.ntis.gov/help/index.asp

Available electronically at http://www.osti.gov/bridge

Available for a processing fee to U.S. Department of Energy and its contractors, in paper, from: U.S. Department of Energy, Office of Scientific and Technical Information, P.O. Box 62, Oak Ridge, TN 37831-0062,

phone: (865)576-8401,

fax: (865)576-5728

email: $\underline{\text { reports@ adonis.osti.gov }}$ 


\section{TABLE OF CONTENTS}

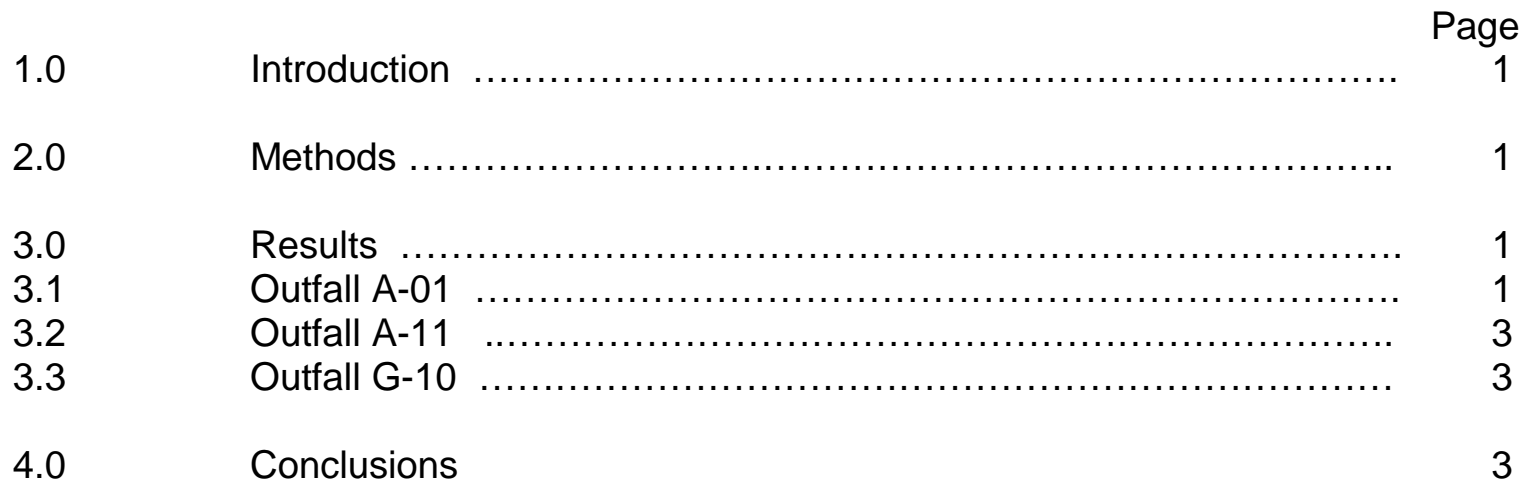

\section{List of Tables}

Table 1 Toxicity Results for SRS NPDES Outfall A-01

2

Table 2 Toxicity Results for SRS NPDES Outfall A-11

4

Table 3 


\subsection{Introduction}

Based on information received in SRS's partial draft NPDES permit (May 2002), monthly chronic definitive toxicity tests will be required at outfalls A-01, A-11 and G-10. Toxicity limits for A-01 and A-11 will be based on 100\% effluent. The toxicity limit for outfall G-10 will be based on a effluent concentration of $19 \%$ for two years, and on an effluent concentration of $100 \%$ for the remainder of the permit.

In October 2001, the Savannah River Site (SRS) received approval from SCDHEC for the use of Daphnia ambigua for routine toxicity testing at SRS. In January 2002, definitive Daphnia ambigua toxicity tests were initiated at outfalls A-01, A-11 and G-10, using SCDHEC's 25/40 method of data analysis (3-parameter logistic regression model). These tests were initiated as a demonstration of "No Reasonable Potential" for toxicity at these outfalls. Communication with SCDHEC indicated that 10 sets of toxicity data for each outfall would be adequate to determine if a reasonable potential still exists for toxicity failures at these outfalls. This report contains the results of these tests.

\subsection{Methods}

The methods used for toxicity testing and data analyses are identical to those specified in the draft NPDES permit. Chronic definitive toxicity tests were conducted using Daphnia ambigua exposed to a laboratory control and the following effluent concentrations: $6.25 \%, 12.5 \%, 25 \%$, $50 \%$ and $100 \%$. The data were analyzed using the 3-parameter logistic regression (3PLR) model assuming a Poisson distribution as recommended in the SCDHEC Bureau of Water document entitled "Options for Data Analysis of Whole Effluent Toxicity Testing Required by NPDES Permits" for calculating biological effect (percent inhibition) at the applicable CTC. Using the 3PLR, toxicity tests are considered to pass if the percent reduction in reproduction averages less than $25 \%$ in any quarter of a calendar year, and no single test in a quarter has a percent reduction that exceeds $40 \%$.

All of the toxicity tests and data analyses were performed by ETT Environmental, Greenville, SC (SCDHEC Certification \# 23104). ETT Environmental was certified by SCDHEC for toxicity testing with Daphnia ambigua in October 2001.

\subsection{Results}

A summary of the results of the 10 sets of toxicity testing is presented in Tables 1 through 3 .

\subsection{Outfall A-01}

The mean reproduction of Daphnia ambigua in 100\% A-01 effluent equaled or exceeded the mean reproduction of the controls in 9 of the 10 tests (Table 1). The data for the week of February 4 indicated that the reproduction in 100\% A-01 effluent was $12.6 \%$ lower than the control, but reproduction rates in the test dilutions were somewhat sporadic (i.e. the reproduction rate in $12.5 \%$ effluent was slightly lower than in $100 \%$ effluent). This variation indicates a weak dose-response relationship, and suggests that the slight impairment in 100\% effluent may not have been due to toxicity. In all 10 data sets; the $25 \%$ Inhibition Concentration (IC25) was $>100 \%$ effluent and the No Observed Effect Concentration (NOEC) was $100 \%$ effluent, which indicates that all 10 tests conducted on A-01 effluent passed. 
Table 1. Toxicity Results for SRS NPDES Outfall A-01

\begin{tabular}{|c|c|c|c|c|c|c|c|c|c|c|c|}
\hline Week of & & Control & $6.25 \%$ & $12.50 \%$ & $25.00 \%$ & $50.00 \%$ & $100 \%$ & Pass/Fail & IC25 & \%Reduction* & NOEC \\
\hline \multirow[t]{2}{*}{$1 / 14 / 2002$} & Reproduction & 21.0 & 21.9 & 18.8 & 22.2 & 18.8 & 21.7 & & & & $100 \%$ \\
\hline & Predicted Values & 20.7 & 20.7 & 20.7 & 20.7 & 20.7 & 20.7 & Pass & $>100 \%$ & $0.0 \%$ & \\
\hline \multirow[t]{2}{*}{$1 / 21 / 2002$} & Reproduction & 25.3 & 21.0 & 25.3 & 26.6 & 25.5 & 26.5 & & & & $100 \%$ \\
\hline & Predicted Values & 24.3 & 24.3 & 24.3 & 24.3 & 24.3 & 24.3 & Pass & $>100 \%$ & $0.0 \%$ & \\
\hline \multirow[t]{2}{*}{ 2/4/2002 } & Reproduction & 30.5 & 29.0 & 25.3 & 26.8 & 28.2 & 26.3 & & & & $100 \%$ \\
\hline & Predicted Values & 30.5 & 27.6 & 27.4 & 27.1 & 26.9 & 26.6 & Pass & $>100 \%$ & $12.6 \%$ & \\
\hline \multirow[t]{2}{*}{$2 / 25 / 2002$} & Reproduction & 26.3 & 25.7 & 24.8 & 26.7 & 27.2 & 26.8 & & & & $100 \%$ \\
\hline & Predicted Values & 26.0 & 26.0 & 26.0 & 26.0 & 26.0 & 26.0 & Pass & $>100 \%$ & $0.0 \%$ & \\
\hline \multirow[t]{2}{*}{$4 / 15 / 2002$} & Reproduction & 25.3 & 25.1 & 24.7 & 26.4 & 27.5 & 27.1 & & & & $100 \%$ \\
\hline & Predicted Values & 22.2 & 22.2 & 22.2 & 22.2 & 22.2 & 22.2 & Pass & $>100 \%$ & $0.0 \%$ & \\
\hline \multirow[t]{2}{*}{$5 / 6 / 2002$} & Reproduction & 25.5 & 28.2 & 29.9 & 29.2 & 27.2 & 27.9 & & & & $100 \%$ \\
\hline & Predicted Values & 28.0 & 28.0 & 28.0 & 28.0 & 28.0 & 28.0 & Pass & $>100 \%$ & $0.0 \%$ & \\
\hline \multirow[t]{2}{*}{$6 / 3 / 2002$} & Reproduction & 20.5 & 20.9 & 19.9 & 27.0 & 23.3 & 24.8 & & & & $100 \%$ \\
\hline & Predicted Values & 22.7 & 22.7 & 22.7 & 22.7 & 22.7 & 22.7 & Pass & $>100 \%$ & $0.0 \%$ & \\
\hline \multirow[t]{2}{*}{$7 / 15 / 02$} & Reproduction & 24.7 & 27.3 & 25.7 & 27.8 & 29.2 & 31 & & & & $100 \%$ \\
\hline & Predicted Values & 27.7 & 27.7 & 27.7 & 27.7 & 27.7 & 27.7 & Pass & $>100 \%$ & $0.0 \%$ & \\
\hline \multirow[t]{2}{*}{$8 / 5 / 2002$} & Reproduction & 24.9 & 25.5 & 27.2 & 29.0 & 24.6 & 25.5 & & & & $100 \%$ \\
\hline & Predicted Values & 26.2 & 26.2 & 26.2 & 26.2 & 26.2 & 26.2 & Pass & $>100 \%$ & $0.0 \%$ & \\
\hline \multirow[t]{2}{*}{ 9/9/2002 } & Reproduction & 22.2 & 21.4 & 23.2 & 20.9 & 22.5 & 22.5 & & & & $100 \%$ \\
\hline & Predicted Values & 22.1 & 22.1 & 22.1 & 22.1 & 22.1 & 22.1 & Pass & $>100 \%$ & $0.0 \%$ & \\
\hline
\end{tabular}




\subsection{Outfall A-11}

The mean reproduction of Daphnia ambigua in $100 \%$ A-11 effluent equaled or exceeded the mean reproduction of the controls in 6 of the 10 tests (Table 2). For the four tests in which reproduction was reduced, reductions ranged from 9.3 to $19.4 \%$. All three tests with reduced reproduction show a weak dose-response relationship (i.e. in the test conducted the week of January 14, control reproduction was slightly lower than reproduction in 100 effluent, but reproduction in intermediate concentrations of effluent were higher; in the test conducted the week of February 4, reproduction rates in $6.25 \%$ effluent and $100 \%$ effluent were almost identical; in September, reproduction was higher in 100\% effluent than in $12.5 \%$ effluent). In all 10 data sets the IC25 was $>100 \%$ effluent and the NOEC was $100 \%$ effluent, which indicates that all 10 tests conducted on A-11 effluent passed.

\subsection{Outfall G-10}

The mean reproduction of Daphnia ambigua in 100\% G-10 effluent equaled or exceeded the mean reproduction of the controls in 5 of the 10 tests (Table 3). Percent reductions in the remaining tests ranged from $6.4 \%$ to $25.2 \%$. With the exception of the test conducted in August, all of the tests conducted on G-10 effluent had NOECs of $100 \%$ effluent and IC25s of $>100 \%$ effluent. In August, the IC25 was $98.8 \%$ effluent, the percent reduction in reproduction in $100 \%$ effluent was $25.2 \%$, and the NOEC was $50 \%$ effluent, although the actual NOEC would have been greater than $50 \%$ if more intermediate dilutions (between $50 \%$ and $100 \%$ ) had been tested. These results indicate that 9 of the 10 tests had a reduction in reproduction that was less than $25 \%$ and that the remaining test was just over $25 \%$. The requirements specified in the draft NPDES permit state that the mean reduction in reproduction for any quarter must be less than $25 \%$, and that reproduction may not be reduced by more than $40 \%$ in any single test. Based on these requirements, the test results for $\mathrm{G}-10$ do not indicate an unacceptable level of toxicity.

\subsection{Conclusions}

The results of the 10 rounds of toxicity tests that were performed on SRS NPDES outfalls A-01, A-11 and G-10 indicate that there would have been no toxicity failures if these tests had been compliance tests. Except for one set of test results for G-10, all of the tests resulted in NOECs of $100 \%$ effluent. All of the tests met the criteria of less than a $25 \%$ mean reduction in reproduction for any quarter and less than a $40 \%$ reduction in any single test. Therefore, there is no reasonable potential to discharge toxic effluents in toxic amounts. 
Table 2. Toxicity Results for SRS NPDES Outfall A-11

\begin{tabular}{|c|c|c|c|c|c|c|c|c|c|c|c|}
\hline Week of & & Control & $6.25 \%$ & $12.50 \%$ & $25.00 \%$ & $50.00 \%$ & $100 \%$ & Pass/Fail & IC25 & \%Reduction* & NOEC \\
\hline \multirow[t]{2}{*}{$1 / 14 / 2002$} & Reproduction & 21.0 & 25.6 & 22.0 & 24.4 & 26.9 & 21.7 & & & & $100 \%$ \\
\hline & Predicted Values & 24.5 & 24.5 & 24.5 & 24.5 & 24.5 & 21.7 & Pass & $>100 \%$ & $9.3 \%$ & \\
\hline \multirow[t]{2}{*}{$1 / 21 / 2002$} & Reproduction & 25.3 & 27.4 & 22.9 & 23.7 & 20.6 & 22.3 & & & & $100 \%$ \\
\hline & Predicted Values & 22.8 & 22.8 & 22.8 & 22.8 & 22.8 & 22.8 & Pass & $>100 \%$ & $0.0 \%$ & \\
\hline \multirow[t]{2}{*}{ 2/4/2002 } & Reproduction & 30.5 & 27.4 & 24.7 & 25.8 & 21.2 & 27.2 & & & & $100 \%$ \\
\hline & Predicted Values & 30.5 & 25.9 & 25.6 & 25.3 & 24.9 & 24.6 & Pass & $>100 \%$ & $19.4 \%$ & \\
\hline \multirow[t]{2}{*}{$2 / 25 / 2002$} & Reproduction & 26.3 & 26.0 & 26.0 & 29.1 & 26.0 & 27.7 & & & & $100 \%$ \\
\hline & Predicted Values & 26.6 & 26.6 & 26.6 & 26.6 & 26.6 & 26.6 & Pass & $>100 \%$ & $0.0 \%$ & \\
\hline \multirow[t]{2}{*}{$4 / 15 / 2002$} & Reproduction & 25.3 & 26.1 & 26.6 & 26.8 & 27.7 & 24.8 & & & & $100 \%$ \\
\hline & Predicted Values & 26.5 & 26.5 & 26.5 & 26.5 & 26.5 & 26.5 & Pass & $>100 \%$ & $0.0 \%$ & \\
\hline \multirow[t]{2}{*}{$5 / 6 / 2002$} & Reproduction & 25.5 & 25.2 & 23.8 & 26.1 & 28.4 & 20.7 & & & & $100 \%$ \\
\hline & Predicted Values & 26.0 & 26.0 & 26.0 & 26.0 & 26.0 & 20.7 & Pass & $>100 \%$ & $15.6 \%$ & \\
\hline \multirow[t]{2}{*}{$6 / 3 / 2002$} & Reproduction & 20.5 & 22.4 & 23.8 & 25.8 & 24.4 & 22.5 & & & & $100 \%$ \\
\hline & Predicted Values & 23.2 & 23.2 & 23.2 & 23.2 & 23.2 & 23.2 & Pass & $>100 \%$ & $0.0 \%$ & \\
\hline \multirow[t]{2}{*}{$7 / 22 / 02$} & Reproduction & 19.3 & 20.7 & 21.3 & 14.1 & 20.0 & 18.6 & & & & $100 \%$ \\
\hline & Predicted Values & 19.0 & 19.0 & 19.0 & 19.0 & 19.0 & 19.0 & Pass & $>100 \%$ & $0.0 \%$ & \\
\hline \multirow[t]{2}{*}{$8 / 5 / 2002$} & Reproduction & 24.9 & 27.1 & 24.8 & 27.5 & 29.3 & 25.9 & & & & $100 \%$ \\
\hline & Predicted Values & 26.6 & 26.6 & 26.6 & 26.6 & 26.6 & 26.6 & Pass & $>100 \%$ & $0.0 \%$ & \\
\hline \multirow[t]{2}{*}{ 9/9/2002 } & Reproduction & 22.2 & 22.7 & 19.2 & 22.8 & 20 & 20.1 & & & & $100 \%$ \\
\hline & Predicted Values & 22.2 & 21.6 & 21.4 & 21.1 & 20.6 & 20 & Pass & $>100 \%$ & $10.0 \%$ & \\
\hline
\end{tabular}


Table 3. Toxicity Results for SRS NPDES Outfall G-10

\begin{tabular}{|c|c|c|c|c|c|c|c|c|c|c|c|}
\hline Week of & & Control & $6.25 \%$ & $12.50 \%$ & $25.00 \%$ & $50.00 \%$ & $100 \%$ & Pass/Fail & IC25 & \%Reduction ${ }^{*}$ & NOEC \\
\hline $1 / 14 / 2002$ & Reproduction & 21.0 & 25.6 & 23.0 & 22.2 & 21.7 & 24.6 & & & & $100 \%$ \\
\hline & Predicted Values & 22.4 & 22.4 & 22.4 & 22.4 & 22.4 & 22.4 & Pass & $>100 \%$ & $0.0 \%$ & \\
\hline $1 / 21 / 2002$ & Reproduction & 25.3 & 23.2 & 27.7 & 25.7 & 24.2 & 25.9 & & & & $100 \%$ \\
\hline & Predicted Values & 24.9 & 24.9 & 24.9 & 24.9 & 24.9 & 24.9 & Pass & $>100 \%$ & $0.0 \%$ & \\
\hline 2/4/2002 & Reproduction & 23.0 & 22.7 & 23.7 & 24.0 & 26.7 & 25.5 & & & & $100 \%$ \\
\hline & Predicted Values & 24.3 & 24.3 & 24.3 & 24.3 & 24.3 & 24.3 & Pass & $>100 \%$ & $0.0 \%$ & \\
\hline $2 / 25 / 2002$ & Reproduction & 26.3 & 25.9 & 21.2 & 23.3 & 28.4 & 28.4 & & & & $100 \%$ \\
\hline & Predicted Values & 25.7 & 25.7 & 25.7 & 25.7 & 25.7 & 25.7 & Pass & $>100 \%$ & $0.0 \%$ & \\
\hline $4 / 22 / 2002$ & Reproduction & 24.4 & 24.8 & 27.2 & 22.3 & 21.9 & 20.5 & & & & $100 \%$ \\
\hline & Predicted Values & 25.3 & 24.9 & 24.5 & 23.8 & 22.5 & 20.1 & Pass & $>100 \%$ & $20.4 \%$ & \\
\hline $5 / 13 / 2002$ & Reproduction & 25.0 & 24.8 & 24.0 & 22.1 & 20.3 & 22.2 & & & & $100 \%$ \\
\hline & Predicted Values & 25.3 & 23.9 & 23.4 & 22.8 & 22.0 & 21 & Pass & $>100 \%$ & $16.9 \%$ & \\
\hline $6 / 3 / 2002$ & Reproduction & 20.5 & 25.2 & 24.4 & 24.3 & 23.4 & 18.6 & & & & $100 \%$ \\
\hline & Predicted Values & 23.6 & 23.6 & 23.6 & 23.6 & 23.4 & 18.6 & Pass & $>100 \%$ & $21.1 \%$ & \\
\hline $7 / 17 / 02$ & Reproduction & 27.0 & 25.5 & 24.7 & 24.5 & 26.9 & 21.5 & & & & $100 \%$ \\
\hline & Predicted Values & 24.2 & 24.2 & 24.2 & 24.2 & 24.2 & 24.2 & Pass & $>100 \%$ & $0.0 \%$ & \\
\hline $8 / 5 / 2002$ & Reproduction & 24.9 & 27.3 & 26.1 & 25.4 & 22.2 & 19.9 & & & & $50 \%$ \\
\hline & Predicted Values & 26.2 & 26.0 & 25.7 & 24.9 & 23.2 & 19.6 & Pass & $98.80 \%$ & $25.2 \%$ & \\
\hline 9/9/2002 & Reproduction & 22.0 & 20.8 & 24.7 & 22.1 & 23.7 & 20.4 & & & & $100 \%$ \\
\hline & Predicted Values & 22.1 & 22.1 & 22.1 & 22.1 & 22.1 & 20.7 & Pass & $>100 \%$ & $6.4 \%$ & \\
\hline
\end{tabular}


APPENDIX 1 


\section{Day Chronic Definitive Survival and Reproduction Bioassay \\ Method: EPA/600/4-91/002}

Test Organism:

Ceriodaphnia dubia

Facility: WSRC

Sample ID: A-01

Aiken County

ETT\#: 19077

Sample ID: January 15, 2002 


\section{CHRONIC DEFINITIVE SURVIVAL AND REPRODUCTION TEST \\ Survival and Reproduction Results}

Client: WSRC

Sample Identification: A-01

Test Date: $\quad$ January 15, 2002

\begin{tabular}{|c|c|c|c|c|c|c|}
\hline \multicolumn{7}{|c|}{ Reproduction at } \\
\hline Rep. & Day 3/4 & Day 5 & Day 6 & Day 7 & Day 8 & Total \\
\hline 1 & 4 & 0 & 11 & 0 & 12 & 27 \\
\hline 2 & 4 & 0 & 8 & 13 & 0 & 25 \\
\hline 3 & 4 & 9 & 0 & 14 & 0 & 27 \\
\hline 4 & 6 & 8 & 13 & 0 & 0 & 27 \\
\hline 5 & 4 & 0 & 4 & $X$ & $\times$ & 8 \\
\hline 6 & 3 & 0 & 2 & 0 & 0 & 5 \\
\hline 7 & 4 & 0 & 10 & 0 & 11 & 25 \\
\hline 8 & 2 & 6 & 0 & 11 & 0 & 19 \\
\hline 9 & 3 & 0 & 7 & 13 & 0 & 23 \\
\hline 10 & 4 & 0 & 8 & 12 & 0 & 24 \\
\hline
\end{tabular}

\begin{tabular}{|c|c|c|c|c|c|c|}
\hline Reproduction at & \multicolumn{4}{c|}{$12.5 \%$} & \multicolumn{3}{c|}{ Concentration } \\
\hline Rep. & Day 3/4 & Day 5 & Day 6 & Day 7 & Day 8 & Total \\
\hline 1 & 3 & 9 & 12 & 0 & 0 & 24 \\
\hline 2 & 3 & 0 & 7 & 13 & 0 & 23 \\
\hline 3 & 5 & 8 & 12 & 0 & 0 & 25 \\
\hline 4 & 3 & 7 & 1 & $X$ & $X$ & 11 \\
\hline 5 & 0 & 0 & $X$ & $X$ & $X$ & 0 \\
\hline 6 & 5 & 9 & 7 & 0 & 0 & 21 \\
\hline 7 & 0 & 4 & 7 & 0 & 11 & 22 \\
\hline 8 & 0 & 9 & $X$ & $X$ & $X$ & 9 \\
\hline 9 & 0 & 8 & 1 & 13 & 12 & 34 \\
\hline 10 & 3 & 3 & 0 & 13 & 0 & 19 \\
\hline
\end{tabular}

\begin{tabular}{|c|c|c|c|c|c|c|}
\hline Reproduction at & \multicolumn{4}{c|}{$50 \%$} & \multicolumn{4}{c|}{ Concentration } \\
\hline Rep. & Day 344 & Day 5 & Day 6 & Day 7 & Day 8 & Tocal \\
\hline 1 & 4 & 9 & 0 & 12 & 0 & 25 \\
\hline 2 & 4 & 7 & 0 & 13 & 0 & 24 \\
\hline 3 & 2 & 9 & 10 & 0 & 0 & 21 \\
\hline 4 & 2 & 0 & 9 & $X$ & $X$ & 11 \\
\hline 5 & 3 & 6 & 0 & 13 & 0 & 22 \\
\hline 6 & 5 & 4 & 12 & 0 & 0 & 21 \\
\hline 7 & 0 & 4 & 9 & 0 & 11 & 24 \\
\hline 8 & 0 & $X$ & $X$ & $X$ & $X$ & 0 \\
\hline 9 & 3 & 5 & 0 & 12 & 0 & 20 \\
\hline 10 & 3 & 4 & 0 & 13 & 0 & 20 \\
\hline
\end{tabular}

County:

NPDES\#:

ETT \#:
Aiken

SC

19077

\begin{tabular}{|c|c|c|c|c|c|c|}
\hline \multicolumn{2}{|c|}{ Reproduction at } & \multicolumn{2}{c|}{$6.25 \%$} & \multicolumn{2}{c|}{ Concentration } \\
\hline Rep. & Day 3/4 & Day 5 & Day 6 & Day 7 & Day 8 & Total \\
\hline 1 & 4 & 7 & 0 & 13 & 0 & 24 \\
\hline 2 & 3 & 0 & 8 & 15 & 0 & 26 \\
\hline 3 & 5 & 5 & 11 & $X$ & $X$ & 21 \\
\hline 4 & 4 & 6 & 0 & 12 & 0 & 22 \\
\hline 5 & 3 & 8 & 0 & 12 & 0 & 23 \\
\hline 6 & 4 & 3 & $X$ & $X$ & $X$ & 7 \\
\hline 7 & 0 & 5 & 10 & 0 & 11 & 26 \\
\hline 8 & 0 & 7 & 11 & $X$ & $X$ & 18 \\
\hline 9 & 3 & 9 & 1 & 12 & 0 & 25 \\
\hline 10 & 4 & 0 & 11 & 0 & 12 & 27 \\
\hline
\end{tabular}

\begin{tabular}{|c|c|c|c|c|c|c|}
\hline \multicolumn{2}{|c|}{ Reproduction at } & \multicolumn{3}{c|}{$25 \%$} & \multicolumn{3}{c|}{ Concentration } \\
\hline Rep. & Day 3/4 & Day 5 & Day 6 & Day 7 & Day 8 & Total \\
\hline 1 & 4 & 9 & 0 & 14 & 0 & 27 \\
\hline 2 & 0 & 0 & 11 & 13 & 0 & 24 \\
\hline 3 & 2 & 8 & 0 & 13 & 0 & 23 \\
\hline 4 & 4 & 7 & $X$ & $X$ & $X$ & 11 \\
\hline 5 & 0 & 6 & 3 & 8 & $X$ & 17 \\
\hline 6 & 5 & 7 & 0 & 15 & 0 & 27 \\
\hline 7 & 0 & 2 & 11 & 0 & 11 & 24 \\
\hline 8 & 0 & 0 & 13 & 0 & 14 & 27 \\
\hline 9 & 2 & 3 & 1 & 14 & 0 & 20 \\
\hline 10 & 0 & 3 & $\mathrm{~L}$ & $\mathrm{~L}$ & $\mathrm{~L}$ & $\mathrm{~L}$ \\
\hline
\end{tabular}

\begin{tabular}{|c|c|c|c|c|c|c}
\hline \multicolumn{2}{|c|}{ Reproduction at } & \multicolumn{2}{c}{$100 \%$} & \multicolumn{3}{c}{ Concentration } \\
\hline Rep. & Day 3/4 & Day 5 & Day 6 & Day 7 & Day 8 & Total \\
\hline 1 & 3 & 0 & $X$ & $X$ & $X$ & 3 \\
\hline 2 & 5 & 0 & 0 & 14 & 13 & 32 \\
\hline 3 & 3 & 6 & 0 & 7 & 0 & 16 \\
\hline 4 & 2 & 5 & 0 & 16 & 0 & 23 \\
\hline 5 & 3 & 7 & 1 & 16 & 0 & 27 \\
\hline 6 & 4 & 8 & 0 & 12 & 0 & 24 \\
\hline 7 & 0 & 8 & 5 & 0 & 5 & 18 \\
\hline 8 & 0 & 4 & 9 & 0 & 13 & 26 \\
\hline 9 & 2 & 7 & 0 & 14 & 0 & 23 \\
\hline 10 & 0 & 9 & 0 & 14 & 2 & 25 \\
\hline
\end{tabular}




\section{CHRONIC DEFINITIVE SURVIVAL AND REPRODUCTION TEST \\ Statistical Analyses}

Client:

WSRC

Sample Identification:

Test Date:

A-01

January 15, 2002

\section{Test for Normality}

Test Used: Shapiro-Wilks

$\begin{array}{ll}W= & N A \\ \text { critical } & N A\end{array}$

NA
Test for Homogeneity of Variance

Test Used: Bartlett's Test

$B=N A$

critical NA

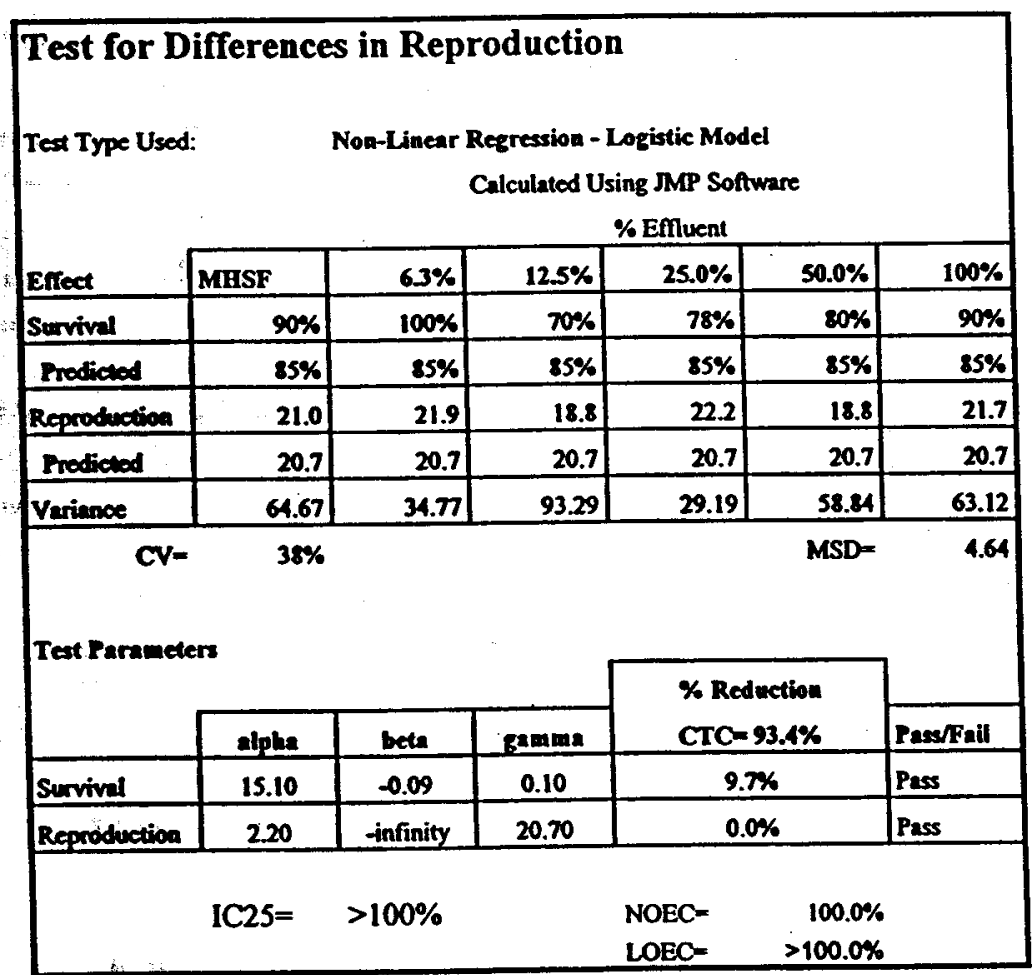

\section{Reproduction vs. Effluent Conc.}

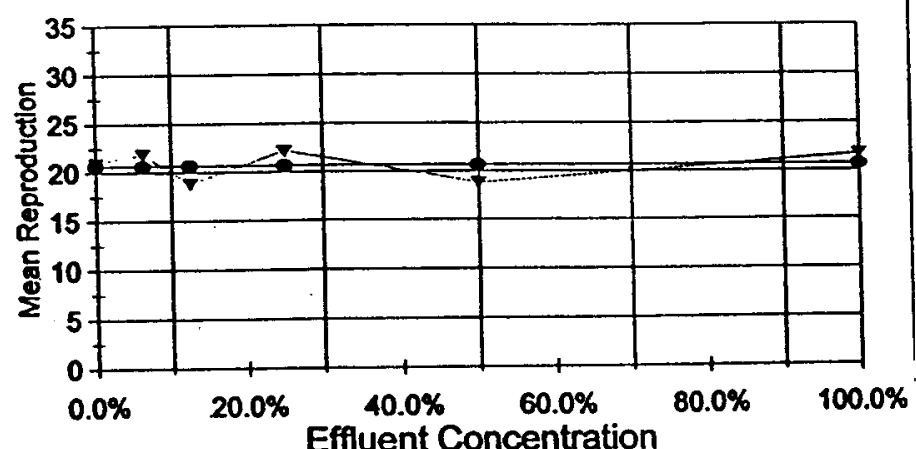

$\rightarrow$ Actual Reproduction $\rightarrow$ Predicted Reproduction

\section{Summary}

The effluent did not reduce reproduction at any test concentration. 


\section{CHRONIC DEFINITIVE SURVIVAL AND REPRODUCTION TEST Water Chemistry}

Client: WSRC

Sample Identification: A-01

Test Date: January 15, 2002

\section{pH (After Renewal)}

\begin{tabular}{|l|r|r|r|r|r|r|}
\hline & \multicolumn{7}{|c|}{ Test Concentration } \\
\hline & Control & $6.25 \%$ & $12.5 \%$ & $25 \%$ & $50 \%$ & $100 \%$ \\
\hline Initial & 7.45 & 7.47 & 7.32 & 7.27 & 7.13 & 7.12 \\
\hline Day.1 & & 7.86 & 7.82 & 7.74 & 7.72 & 7.68 \\
\hline Day 2 & 7.59 & 7.24 & 7.10 & 7.05 & 6.97 & 7.04 \\
\hline Day 3 & 7.65 & 7.51 & 7.44 & 7.38 & 7.31 & 7.29 \\
\hline Day 4 & 6.90 & 6.90 & 6.95 & 7.10 & 7.06 & 7.10 \\
\hline Day 5 & 8.31 & 8.47 & 8.35 & 8.23 & 8.12 & 7.89 \\
\hline Day 6 & 7.52 & 7.43 & 7.40 & 7.39 & 7.36 & 7.40 \\
\hline
\end{tabular}

Dissolved Oxygen (After Renewal)

\begin{tabular}{|l|r|r|r|r|r|r|}
\hline & Control & $6.25 \%$ & $12.5 \%$ & $25 \%$ & $50 \%$ & $100 \%$ \\
\hline & & 8.6 & 8.5 & 8.5 & 8.6 & 8.6 \\
\hline Lnitial & & 8.6 & 8.6 & 8.6 & 8.7 & 8.7 \\
\hline Day 1 & 8.9 & 8.7 & 8.7 & 8.7 & 8.8 & 8.9 \\
\hline Day 2 & 8.9 & 8.6 & 8.9 & 8.8 & 8.9 & 9.0 \\
\hline Day 3 & 8.9 & 8.8 & 8.9 & 8.9 & 9.0 & 9.1 \\
\hline Day 4 & 8.8 & 8.8 & 8.8 & 8.8 & 8.8 & 8.9 \\
\hline Day 5 & & & & & & \\
\hline Day 6 & & & & & \\
\hline
\end{tabular}

Test Results Reviewed and Approved By:

pH (Before Renewal)
\begin{tabular}{|l|r|r|r|r|r|r|}
\hline \multicolumn{7}{|c|}{ Test Concentration } \\
\hline & Control & $6.25 \%$ & $12.5 \%$ & $25 \%$ & $50 \%$ & $100 \%$ \\
\hline Day 1 & & 7.11 & 7.17 & 7.20 & 7.31 & 7.36 \\
\hline Day 2 & 7.68 & 7.53 & 7.48 & 7.42 & 7.43 & 7.45 \\
\hline Day 3 & 8.00 & 7.87 & 7.72 & 7.64 & 7.61 & 7.62 \\
\hline Day 4 & 6.25 & 7.16 & 7.19 & 7.12 & 7.26 & 7.33 \\
\hline Day 5 & 8.41 & 8.61 & 8.50 & 8.34 & 8.27 & 8.10 \\
\hline Day 6 & 7.82 & 7.78 & 7.74 & 7.72 & 7.72 & 7.82 \\
\hline Final & 7.77 & 7.39 & 7.30 & 7.28 & 7.32 & 7.42 \\
\hline
\end{tabular}

\section{Dissolved Oxygen (Before Renewal)}

\begin{tabular}{|l|r|r|r|r|r|r|}
\hline & Control & $6.25 \%$ & $12.5 \%$ & $25 \%$ & $50 \%$ & $100 \%$ \\
\hline Day 1 & & 8.4 & 8.4 & 8.3 & 8.3 & 8.3 \\
\hline Day 2 & 8.5 & 8.6 & 8.6 & 8.5 & 8.6 & 8.6 \\
\hline Day 3 & 8.9 & 8.8 & 8.9 & 9.1 & 9.0 & 9.1 \\
\hline Day 4 & 8.8 & 8.7 & 8.9 & 9.0 & 9.0 & 9.0 \\
\hline Day 5 & 8.8 & 8.8 & 8.8 & 8.8 & 8.8 & 8.8 \\
\hline Day 6 & 8.8 & 8.8 & 8.8 & 8.8 & 8.8 & 8.8 \\
\hline Final & 8.6 & 8.6 & 8.6 & 8.8 & 8.9 & 9.0 \\
\hline
\end{tabular}

Temperature
\begin{tabular}{|l|r|}
\hline \multicolumn{2}{|c|}{ Incubator ${ }^{\circ} \mathrm{C}$} \\
\hline Initial & 25.0 \\
\hline Day 1 & 24.2 \\
\hline Day 2 & 24.9 \\
\hline Day 3 & 25.3 \\
\hline Day 4 & 24.5 \\
\hline Day 5 & 24.0 \\
\hline Day 6 & 24.0 \\
\hline Final & 24.5 \\
\hline
\end{tabular}




\section{Day Chronic Definitive Survival and Reproduction Bioassay \\ Method: EPA/600/4-91/002}

Test Organism:

Ceriodaphnia dubia

Facility: WSRC

Sample ID: A-11

Aiken County

ETTH: 19078

Sample ID: January 15, 2002 


\section{CHRONIC DEFINITIVE SURVIVAL AND REPRODUCTION TEST}

\section{Survival and Reproduction Results}

Client:

WSRC

Sample Identification:

Test Date:

A-11

January 15, 2002
County:

NPDESH:

ETT \#:
Aiken

SC

19078

\begin{tabular}{|c|c|c|c|c|c|c|}
\hline \multicolumn{2}{|c|}{ Reproduction at } & \multicolumn{7}{c|}{ MHSF Control } \\
\hline Rep. & Day 3/4 & Day 5 & Day6 & Day 7 & Day 8 & Total \\
\hline 1 & 4 & 0 & 11 & 0 & 12 & 27 \\
\hline 2 & 4 & 0 & 8 & 13 & 0 & 25 \\
\hline 3 & 4 & 9 & 0 & 14 & 0 & 27 \\
\hline 4 & 6 & 8 & 13 & 0 & 0 & 27 \\
\hline 5 & 4 & 0 & 4 & $X$ & $X$ & 8 \\
\hline 6 & 3 & 0 & 2 & 0 & 0 & 5 \\
\hline 7 & 4 & 0 & 10 & 0 & 11 & 25 \\
\hline 8 & 2 & 6 & 0 & 11 & 0 & 19 \\
\hline 9 & 3 & 0 & 7 & 13 & 0 & 23 \\
\hline 10 & 4 & 0 & 8 & 12 & 0 & 24 \\
\hline
\end{tabular}

\begin{tabular}{|c|c|c|c|c|c|c|}
\hline \multicolumn{3}{|c|}{ Reproduction at } & \multicolumn{9}{|c|}{$6.25 \%$} & \multicolumn{3}{c|}{ Concentration } \\
\hline Rep. & Day 3/4 & Day 5 & Day 6 & Day 7 & Day 8 & Total \\
\hline 1 & 3 & 10 & 0 & 16 & 0 & 29 \\
\hline 2 & 4 & 6 & 0 & 13 & 0 & 23 \\
\hline 3 & 5 & 9 & 0 & 13 & 0 & 27 \\
\hline 4 & 3 & 8 & 0 & 15 & 0 & 26 \\
\hline 5 & 3 & 10 & 0 & 12 & 0 & 25 \\
\hline 6 & 6 & 0 & 9 & 0 & 18 & 33 \\
\hline 7 & 6 & 0 & 11 & 0 & 18 & 35 \\
\hline 8 & 0 & 7 & 0 & $X$ & $X$ & 7 \\
\hline 9 & 2 & 6 & 0 & 13 & 0 & 21 \\
\hline 10 & 0 & 7 & 0 & 10 & 13 & 30 \\
\hline
\end{tabular}

\begin{tabular}{|c|c|c|c|c|c|c|}
\hline \multicolumn{2}{|c|}{ Reproduction at } & \multicolumn{3}{c|}{$12.5 \%$} & \multicolumn{2}{c|}{ Concentration } \\
\hline Rep. & Day 3/4 & Day 5 & Day 6 & Day 7 & Day 8 & Total \\
\hline 1 & 10 & 0 & 10 & 0 & 0 & 20 \\
\hline 2 & 5 & 8 & 0 & 12 & 0 & 25 \\
\hline 3 & 7 & 9 & 0 & 12 & 0 & 28 \\
\hline 4 & 4 & 7 & 0 & 11 & 0 & 22 \\
\hline 5 & 3 & 7 & 0 & 11 & 0 & 21 \\
\hline 6 & 6 & 7 & 0 & 12 & 0 & 25 \\
\hline 7 & 4 & 9 & 0 & 14 & 0 & 27 \\
\hline 8 & $X$ & $X$ & $X$ & $X$ & $X$ & 0 \\
\hline 9 & 2 & 10 & 0 & 13 & 0 & 25 \\
\hline 10 & 3 & 9 & 0 & 15 & 0 & 27 \\
\hline
\end{tabular}

\begin{tabular}{|c|c|c|c|c|c|c|}
\hline \multicolumn{3}{|c|}{ Reproduction at } & \multicolumn{3}{c|}{$25 \%$} & \multicolumn{3}{c|}{ Concentration } \\
\hline Rep. & Day 3/4 & Day 5 & Day 6 & Day 7 & Day 8 & Tocal \\
\hline 1 & 8 & 1 & 8 & 0 & 10 & 27 \\
\hline 2 & 3 & 10 & 0 & 13 & 0 & 26 \\
\hline 3 & 6 & 10 & 0 & 12 & 0 & 28 \\
\hline 4 & 3 & 10 & 0 & 12 & 0 & 25 \\
\hline 5 & 3 & 7 & 0 & 13 & 0 & 23 \\
\hline 6 & 4 & 6 & 0 & 7 & 0 & 17 \\
\hline 7 & 0 & 6 & 9 & 0 & 0 & 15 \\
\hline 8 & 7 & 1 & 10 & 0 & 18 & 36 \\
\hline 9 & 2 & 8 & 0 & 14 & 0 & 24 \\
\hline 10 & 2 & 8 & 0 & 13 & 0 & 23 \\
\hline
\end{tabular}

\begin{tabular}{|c|c|c|c|c|c|c|}
\hline \multicolumn{2}{|c|}{ Reproduction at } & \multicolumn{3}{|c|}{$50 \%$} & \multicolumn{3}{c|}{ Concentration } \\
\hline Rep. & Day 3/4 & Day 5 & Day 6 & Day 7 & Day 8 & Tolal \\
\hline 1 & 7 & 0 & 12 & 0 & 18 & 37 \\
\hline 2 & 5 & 10 & 0 & 13 & 0 & 28 \\
\hline 3 & 2 & 10 & 0 & 13 & 0 & 25 \\
\hline 4 & 5 & 10 & 0 & 14 & 0 & 29 \\
\hline 5 & 5 & 0 & 8 & $X$ & $X$ & 13 \\
\hline 6 & 3 & 8 & 0 & 12 & 0 & 23 \\
\hline 7 & 7 & 0 & 12 & 0 & 16 & 35 \\
\hline 8 & 6 & 0 & 11 & 0 & 10 & 27 \\
\hline 9 & 2 & 8 & 0 & 14 & 0 & 24 \\
\hline 10 & 4 & 10 & 0 & 14 & 0 & 28 \\
\hline
\end{tabular}

\begin{tabular}{|c|c|c|c|c|c|c}
\hline \multicolumn{3}{|c|}{ Reproduction at } & \multicolumn{3}{c}{$100 \%$} & \multicolumn{2}{c}{ Concentration } \\
\hline Rep. & Day 3/4 & Day 5 & Day 6 & Day 7 & Day 8 & Total \\
\hline 1 & 3 & 9 & 0 & 13 & 0 & 25 \\
\hline 2 & 2 & 10 & 0 & 15 & 0 & 27 \\
\hline 3 & 4 & 8 & 0 & 13 & 0 & 25 \\
\hline 4 & 3 & 8 & 0 & 12 & 0 & 23 \\
\hline 5 & 6 & 2 & 10 & 0 & 0 & 18 \\
\hline 6 & 3 & 4 & 10 & 0 & 0 & 17 \\
\hline 7 & 9 & 0 & 9 & 0 & 0 & 18 \\
\hline 8 & 7 & 2 & 11 & 0 & 0 & 20 \\
\hline 9 & 0 & 9 & 0 & 14 & $X$ & 23 \\
\hline 10 & 9 & 0 & 12 & 0 & 0 & 21 \\
\hline
\end{tabular}




\section{CHRONIC DEFINITIVE SURVIVAL AND REPRODUCTION TEST \\ Statistical Analyses}

Client:

Sample Identification:

Test Date:
WSRC

A-11

January 15,2002

\begin{tabular}{|cll|}
\hline Test for Normality & & \\
Test Used: Kolmogorov D & $\begin{array}{ll} \\
\text { critical }\end{array}$ & NA \\
NA & & \\
& & \\
\hline
\end{tabular}

Test for Homogeneity of Variance

Test Used: Bartlett's Test

$B=N A$

critical NA

NA

\begin{tabular}{|c|c|c|c|c|c|c|}
\hline \multicolumn{7}{|c|}{ Test for Differences in Reproduction } \\
\hline \multicolumn{2}{|c|}{ Test Type Used: } & \multicolumn{5}{|c|}{$\begin{array}{c}\text { Non-Linear Regression - Logistic Model } \\
\text { Calculated Using JMP Software } \\
\text { \% Effluent }\end{array}$} \\
\hline Effect & MHSF & $6.3 \%$ & $12.5 \%$ & $25.0 \%$ & $50.0 \%$ & $100 \%$ \\
\hline Survival & $90 \%$ & $100 \%$ & $90 \%$ & $100 \%$ & $90 \%$ & $90 \%$ \\
\hline Prodictod & $100 \%$ & $100 \%$ & $100 \%$ & $100 \%$ & $100 \%$ & $100 \%$ \\
\hline Reproduction & 21.0 & 25.6 & 22.0 & 24.4 & 26.9 & 21.7 \\
\hline Prodicted & 24.5 & 24.5 & 24.5 & 24.5 & 24.5 & 21.7 \\
\hline Variance & 64.67 & 61.16 & 66.89 & 33.82 & 43.88 & 11.79 \\
\hline Test Parames & $38 \%$ & & & & MSD= & 7.08 \\
\hline & alpha & beta & gamma & \multicolumn{2}{|c|}{$\begin{array}{l}\text { X Reduction } \\
\text { CTC= } 98.1 \%\end{array}$} & Pessoffail \\
\hline Survivel & 3.00 & -200.00 & 0.00 & \multicolumn{2}{|c|}{$0.0 \%$} & Pass \\
\hline Reproduction & 2.07 & -28.80 & 24.50 & \multicolumn{2}{|c|}{ 9.3\% } & Pass \\
\hline \multicolumn{4}{|c|}{ IC25 $=$} & $\begin{array}{l}\text { NOEC }= \\
\text { LOEC }=\end{array}$ & $\begin{array}{r}100.00 \% \\
100 \% \\
\end{array}$ & \\
\hline
\end{tabular}

Reproduction vs. Effluent Conc.

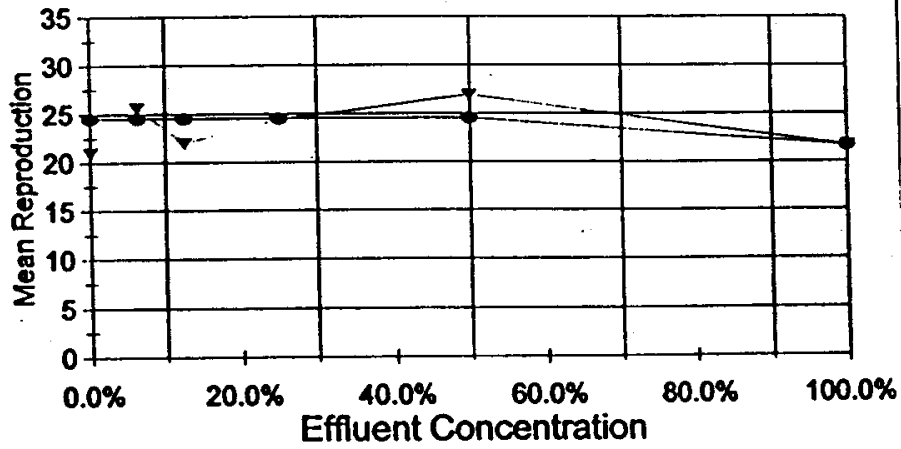

$\rightarrow$ Actual Reproduction $\rightarrow$ Predicted Reproduction

Summary

There was a small reduction in reproduction at the CTC of $98.1 \%$, but less than the average limit of $25 \%$. 


\section{CHRONIC DEFINITIVE SURVIVAL AND REPRODUCTION TEST Water Chemistry}

Client: $\quad$ WSRC

Sample Identification: A-11

Test Date: $\quad$ January 15, 2002

pH (After Renewal)

\begin{tabular}{|l|r|r|r|r|r|r|}
\hline \multicolumn{7}{|c|}{ Test Concentration } \\
\hline & Control & $6.25 \%$ & $12.5 \%$ & $25 \%$ & $50 \%$ & $100 \%$ \\
\hline Initial & 7.45 & 8.22 & 8.30 & 7.87 & 7.69 & 7.60 \\
\hline Day 1 & & 8.63 & 8.84 & 8.50 & 8.33 & 8.08 \\
\hline Day 2 & 7.59 & 7.05 & 6.89 & 6.88 & 6.83 & 6.91 \\
\hline Day 3 & & 8.71 & 8.16 & 8.01 & 8.03 & 8.02 \\
\hline Day 4 & 6.90 & 7.23 & 7.28 & 7.39 & 7.49 & 7.52 \\
\hline Day 5 & & 7.47 & 7.86 & 7.74 & 7.70 & 7.64 \\
\hline Day 6 & & 7.62 & 7.50 & 7.44 & 7.37 & 7.31 \\
\hline
\end{tabular}

Dissolved Oxygen (After Renewal)

\begin{tabular}{|l|r|r|r|r|r|r|}
\hline \multicolumn{7}{|c|}{ Test Concentration } \\
\hline & Control & $6.25 \%$ & $12.5 \%$ & $25 \%$ & $50 \%$ & $100 \%$ \\
\hline Initial & & 8.5 & 8.5 & 8.5 & 8.5 & 8.6 \\
\hline Day 1 & & 8.7 & 8.7 & 8.6 & 8.6 & 8.5 \\
\hline Day 2 & 8.9 & 8.8 & 8.8 & 8.7 & 8.7 & 8.5 \\
\hline Day 3 & & 9.0 & 8.1 & 9.0 & 9.0 & 8.9 \\
\hline Day 4 & 8.9 & 9.0 & 9.0 & 8.9 & 8.9 & 9.1 \\
\hline Day 5 & & 8.8 & 8.8 & 8.6 & 8.7 & 8.7 \\
\hline Day 6 & & 8.8 & 8.8 & 8.7 & 8.9 & 9.0 \\
\hline
\end{tabular}

Test Results Reviewed and Approved By:

\section{Dissolved Oxygen (Before Renewal)}

\begin{tabular}{|l|r|r|r|r|r|r|}
\hline \multicolumn{7}{|c|}{ Test Concentration } \\
\hline & Control & $6.25 \%$ & $12.5 \%$ & $25 \%$ & $50 \%$ & $100 \%$ \\
\hline Day 1 & & 8.2 & 8.2 & 8.1 & 8.1 & 8.0 \\
\hline Day 2 & 8.5 & 8.4 & 8.2 & 8.5 & 8.5 & 8.5 \\
\hline Day 3 & & 8.6 & 8.6 & 8.6 & 8.6 & 8.6 \\
\hline Day 4 & 8.9 & 8.8 & 8.9 & 9.0 & 9.1 & 9.1 \\
\hline Day 5 & & 8.7 & 8.7 & 8.8 & 8.8 & 8.7 \\
\hline Day 6 & & 8.8 & 8.9 & 8.9 & 8.9 & 8.9 \\
\hline Final & 8.6 & 8.4 & 8.5 & 8.7 & 8.7 & 8.9 \\
\hline
\end{tabular}

pH (Before Renewal)

\begin{tabular}{|l|r|r|r|r|r|r|}
\hline \multicolumn{7}{|c|}{ Test Concentration } \\
\hline & Control & $6.25 \%$ & $12.5 \%$ & $25 \%$ & $50 \%$ & $100 \%$ \\
\hline Day 1 & & 7.41 & 7.26 & 7.20 & 7.14 & 7.17 \\
\hline Day 2 & 7.68 & 7.42 & & 7.38 & 7.35 & 7.37 \\
\hline Day 3 & & 8.72 & 8.72 & 8.25 & 8.27 & 8.19 \\
\hline Day 4 & 6.25 & 6.88 & 6.99 & 7.14 & 7.33 & 7.40 \\
\hline Day 5 & & 8.07 & 8.01 & 7.90 & 7.88 & 7.88 \\
\hline Day 6 & & 7.82 & 7.55 & 7.66 & 7.57 & 7.60 \\
\hline Final & 7.77 & 7.53 & 7.63 & 7.44 & 7.36 & 7.39 \\
\hline
\end{tabular}

Temperature

\begin{tabular}{|l|r|}
\hline \multicolumn{1}{|c|}{ Incubator ${ }^{\circ} \mathrm{C}$} \\
\hline Initial & 25.0 \\
\hline Day 1 & 24.2 \\
\hline Day 2 & 24.9 \\
\hline Day 3 & 25.3 \\
\hline Day 4 & 24.5 \\
\hline Day 5 & 24.0 \\
\hline Day 6 & 24.0 \\
\hline Final & 24.5 \\
\hline
\end{tabular}




\section{Day Chronic Definitive Survival and Reproduction Bioassay Method: EPA/600/4-91/002}

Test Organism:

Ceriodaphnia dubia

Facility: WSRC

Sample ID: G-10

Aiken County

ETT\#: 19079 


\section{CHRONIC DEFINITIVE SURVIVAL AND REPRODUCTION TEST \\ Survival and Reproduction Results}

Client: WSRC

Sample Identification:

Test Date:

G-10

January 15, 2002
County:

NPDES\#:

ETT \#:
Aiken

SC

19079

\begin{tabular}{|c|c|c|c|c|c|c|}
\hline \multicolumn{7}{|c|}{ Reproduction at } \\
\hline Rep. & Day 3/4 & Day 5 & Day 6 & Day 7 & Day 8 & Toual \\
\hline 1 & 4 & 0 & 11 & 0 & 12 & 27 \\
\hline 2 & 4 & 0 & 8 & 13 & 0 & 25 \\
\hline 3 & 4 & 9 & 0 & 14 & 0 & 27 \\
\hline 4 & 6 & 8 & 13 & 0 & 0 & 27 \\
\hline 5 & 4 & 0 & 4 & $X$ & $X$ & 8 \\
\hline 6 & 3 & 0 & 2 & 0 & 0 & 5 \\
\hline 7 & 4 & 0 & 10 & 0 & 11 & 25 \\
\hline 8 & 2 & 6 & 0 & 11 & 0 & 19 \\
\hline 9 & 3 & 0 & 7 & 13 & 0 & 23 \\
\hline 10 & 4 & 0 & 8 & 12 & 0 & 24 \\
\hline
\end{tabular}

\begin{tabular}{|c|c|c|c|c|c|c|}
\hline \multicolumn{2}{|c|}{ Reproduction at } & \multicolumn{2}{c|}{$6.25 \%$} & \multicolumn{3}{c|}{ Concentration } \\
\hline Rep. & Day 3/4 & Day 5 & Day 6 & Day 7 & Day 8 & Total \\
\hline 1 & 6 & 0 & 11 & 10 & 0 & 27 \\
\hline 2 & 4 & 9 & 0 & 12 & 0 & 25 \\
\hline 3 & 3 & 11 & 0 & 13 & 0 & 27 \\
\hline 4 & 0 & 7 & 0 & 11 & 0 & 18 \\
\hline 5 & 3 & $X$ & $X$ & $X$ & $X$ & 3 \\
\hline 6 & 3 & 7 & 0 & 12 & 0 & 22 \\
\hline 7 & 0 & 0 & 8 & 0 & 10 & 18 \\
\hline 8 & 3 & 6 & 0 & 13 & 0 & 22 \\
\hline 9 & 4 & 12 & 0 & 14 & 0 & 30 \\
\hline 10 & 8 & 0 & 12 & 0 & 18 & 38 \\
\hline
\end{tabular}

\begin{tabular}{|c|c|c|c|c|c|c|}
\hline Reproduction at & \multicolumn{4}{c|}{$12.5 \%$} & \multicolumn{3}{c|}{ Concentration } \\
\hline Rep. & Day 3/4 & Day 5 & Day 6 & Day 7 & Day & Total \\
\hline 1 & 4 & 0 & 11 & 12 & 0 & 27 \\
\hline 2 & 4 & 10 & 0 & 16 & 0 & 30 \\
\hline 3 & 4 & 8 & 0 & 10 & 0 & 22 \\
\hline 4 & 0 & 0 & 0 & 0 & 0 & 0 \\
\hline 5 & 4 & 0 & $X$ & $X$ & $X$ & 4 \\
\hline 6 & 5 & 0 & 12 & 0 & 15 & 32 \\
\hline 7 & 4 & 10 & 0 & 14 & 0 & 28 \\
\hline 8 & 3 & 9 & 0 & 14 & 0 & 26 \\
\hline 9 & 6 & 10 & 0 & 13 & 0 & 29 \\
\hline 10 & 5 & 0 & 11 & 0 & 16 & 32 \\
\hline
\end{tabular}

\begin{tabular}{|c|c|c|c|c|c|c|}
\hline \multicolumn{2}{|c|}{ Reproduction at } & \multicolumn{2}{c|}{$25 \%$} & \multicolumn{2}{c|}{ Concentration } \\
\hline Rep. & Day 3/4 & Day 5 & Day 6 & Day 7 & Day 8 & Toual \\
\hline 1 & 5 & 0 & 9 & 16 & 0 & 30 \\
\hline 2 & 6 & 0 & 9 & 0 & 14 & 29 \\
\hline 3 & $X$ & $X$ & $X$ & $X$ & $X$ & 0 \\
\hline 4 & 4 & 11 & 12 & 0 & 0 & 27 \\
\hline 5 & 6 & 0 & 13 & 0 & 15 & 34 \\
\hline 6 & 0 & 9 & 0 & 13 & 12 & 34 \\
\hline 7 & 4 & 10 & 0 & 15 & 0 & 29 \\
\hline 8 & 1 & 0 & 3 & 10 & 0 & 14 \\
\hline 9 & $X$ & $X$ & $X$ & $X$ & $X$ & 0 \\
\hline 10 & 3 & 10 & 0 & 12 & 0 & 25 \\
\hline
\end{tabular}

\begin{tabular}{|c|c|c|c|c|c|c|}
\hline Reproduction at & \multicolumn{4}{c|}{$50 \%$} & \multicolumn{3}{c|}{ Concentration } \\
\hline Rep. & Day 3/4 & Day 5 & Day 6 & Day 7 & Day 8 & Toul \\
\hline 1 & 3 & 0 & 9 & 10 & 0 & 22 \\
\hline 2 & 3 & 9 & 0 & 12 & 0 & 24 \\
\hline 3 & 4 & 10 & 0 & 13 & 0 & 27 \\
\hline 4 & 3 & 8 & 0 & 13 & 0 & 24 \\
\hline 5 & 4 & 6 & 0 & $X$ & $X$ & 10 \\
\hline 6 & 9 & 0 & 13 & 0 & 0 & 22 \\
\hline 7 & 1 & 10 & 0 & 10 & 0 & 21 \\
\hline 8 & 1 & 10 & 0 & 13 & 0 & 24 \\
\hline 9 & 3 & 6 & 0 & 10 & 0 & 19 \\
\hline 10 & 4 & 8 & 0 & 12 & 0 & 24 \\
\hline
\end{tabular}

\begin{tabular}{|c|c|c|c|c|c|c}
\hline Reproduction at & \multicolumn{2}{c}{$100 \%$} & \multicolumn{3}{c}{ Concentration } \\
\hline Rep. & Day 3/4 & Day 5 & Day 6 & Day 7 & Day 8 & Total \\
\hline 1 & 3 & 0 & 10 & 13 & 0 & 26 \\
\hline 2 & 4 & 6 & 0 & 12 & 0 & 22 \\
\hline 3 & 6 & 0 & 9 & 0 & 13 & 28 \\
\hline 4 & 3 & 8 & 0 & 13 & 0 & 24 \\
\hline 5 & 4 & 9 & 0 & 14 & 0 & 27 \\
\hline 6 & 0 & 6 & 0 & 10 & 0 & 16 \\
\hline 7 & 0 & 8 & 0 & 8 & 0 & 16 \\
\hline 8 & 0 & 7 & 6 & 2 & 12 & 27 \\
\hline 9 & 0 & 10 & 0 & 13 & 14 & 37 \\
\hline 10 & 4 & 7 & 0 & 12 & 0 & 23 \\
\hline
\end{tabular}




\section{CHRONIC DEFINITIVE SURVIVAL AND REPRODUCTION TEST \\ Statistical Analyses}

Client: WSRC

Sample Identification: $\quad$ G-10

Test Date:

January 15, 2002

\section{Test for Normality}

Test Used: Kolmogorov D

$\begin{array}{ll}D= & N A \\ \text { critical } & \text { NA }\end{array}$

NA

\section{Test for Differences in Reproduction}

\begin{tabular}{|c|c|c|c|c|c|c|}
\hline \multicolumn{2}{|c|}{ Tex Type Used: } & \multicolumn{5}{|c|}{$\begin{array}{l}\text { Non-Linear Regression - Logistic Model } \\
\text { Calculated Using MMP Software } \\
\text { \% Effluent }\end{array}$} \\
\hline Enteet & MHSF & $6.3 \%$ & $12.5 \%$ & $25.0 \%$ & $50.0 \%$ & $100 \%$ \\
\hline Survivel. & $90 \%$ & $90 \%$ & $90 \%$ & $80 \%$ & $90 \%$ & $100 \%$ \\
\hline Procicend & $90 \%$ & $90 \%$ & $90 \%$ & $90 \%$ & $90 \%$ & $90 \%$ \\
\hline Reproduction & 21.0 & 25.6 & 23.0 & 22.2 & 21.7 & 24.6 \\
\hline Prodicted & 22.4 & 22.4 & 22.4 & 22.4 & 22.4 & 22.4 \\
\hline Variemoce & 64.67 & 21.78 & 132.00 & 168.40 & 21.57 & 37.38 \\
\hline CV= & $36 \%$ & & & & MSD $=$ & 8.96 \\
\hline
\end{tabular}

Tex Farametera

\begin{tabular}{|c|c|c|c|c|c|c|}
\hline \multicolumn{4}{|c|}{ 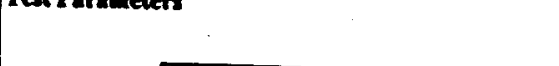 } & \multirow{2}{*}{\multicolumn{2}{|c|}{$\begin{array}{c}\text { \% Roduction } \\
\text { CTC- } 19 \% \text { CTC-100\% }\end{array}$}} & \multirow[b]{2}{*}{ PaserFail } \\
\hline & alpha & beta & gamma & & & \\
\hline Survival & 3.00 & -200.00 & 0.10 & $0.0 \%$ & $0.0 \%$ & Pass \\
\hline \multirow[t]{2}{*}{ Reproduction } & 3.28 & -1.57 & 22.40 & $0.0 \%$ & $0.0 \%$ & Pass \\
\hline & IC2S $=$ & $>100 \%$ & & $\begin{array}{l}\text { NOEC= } \\
\text { LOEC= }\end{array}$ & $\begin{array}{r}100.0 \% \\
>100 \% \\
\end{array}$ & \\
\hline
\end{tabular}

Test for Homogeneity of Variance

Test Used: Bartlett's Test

$$
B=N A
$$

critical NA

NA
Reproduction vs. Effluent Conc.

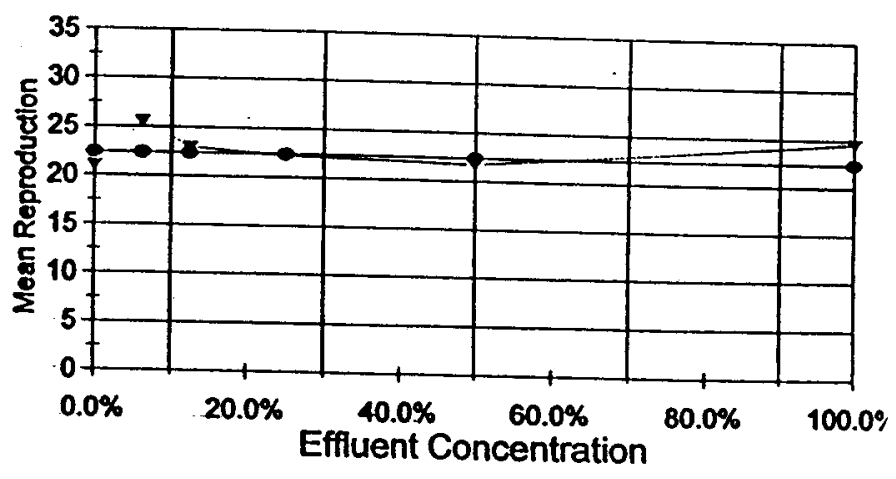

- - Actual Reproduction $\rightarrow$ Predicted Reproduction

Summary

The effluent did not reduce reproduction or survival at any test concentration. 


\section{CHRONIC DEFINITIVE SURVIVAL AND REPRODUCTION TEST \\ Water Chemistry}

Client: $\quad$ WSRC

Sample Identification: $\quad$ G-10

Test Date: January 15, 2002

pH (After Renewal)

\begin{tabular}{|l|r|r|r|r|r|r|}
\hline \multicolumn{7}{|c|}{ Test Concentration } \\
\hline & Control & $6.25 \%$ & $12.5 \%$ & $25 \%$ & $50 \%$ & $100 \%$ \\
\hline Initial & 7.45 & 8.53 & 8.30 & 8.14 & 7.96 & 7.87 \\
\hline Day 1 & & 7.66 & 7.54 & 7.51 & 7.69 & 7.96 \\
\hline Day 2 & 7.59 & 7.09 & 7.05 & 7.12 & 7.33 & 7.79 \\
\hline Day 3 & & 7.64 & 7.57 & 7.61 & 7.67 & 7.93 \\
\hline Day 4 & 6.90 & 7.57 & 7.59 & 7.65 & 7.74 & 7.85 \\
\hline Day 5 & & 7.49 & 7.51 & 7.54 & 8.05 & 8.19 \\
\hline Day 6 & & 7.67 & 7.62 & 7.63 & 7.78 & 7.97 \\
\hline
\end{tabular}

Dissolved Oxygen (After Renewal)

\begin{tabular}{|l|r|r|r|r|r|r|}
\hline & \multicolumn{7}{|c|}{ Test Concentration } \\
\hline & & $6.25 \%$ & $12.5 \%$ & $25 \%$ & $50 \%$ & $100 \%$ \\
\hline Initial & 8.6 & 8.6 & 8.6 & 8.6 & 8.6 \\
\hline Day 1 & & 8.7 & 8.7 & 8.7 & 8.8 & 8.8 \\
\hline Day 2 & 8.9 & 8.5 & 8.6 & 8.7 & 8.8 & 8.8 \\
\hline Day 3 & & 8.6 & 8.6 & 8.6 & 8.6 & 8.6 \\
\hline Day 4 & 8.9 & 8.4 & 8.4 & 8.6 & 8.6 & 8.6 \\
\hline Day 5 & & 8.5 & 8.5 & 8.5 & 8.6 & 8.6 \\
\hline Day 6 & & 8.6 & 8.6 & 8.7 & 8.8 & 8.8 \\
\hline
\end{tabular}

pH (Before Renewal)

\begin{tabular}{|l|r|r|r|r|r|r|}
\hline \multicolumn{7}{|c|}{ Test Concentration } \\
\hline & Control & $6.25 \%$ & $12.5 \%$ & $25 \%$ & $50 \%$ & $100 \%$ \\
\hline Day 1 & & 7.74 & 7.50 & 7.74 & 7.78 & 7.96 \\
\hline Day 2 & 7.68 & 7.41 & 7.38 & 7.37 & 7.46 & 7.95 \\
\hline Day 3 & & 7.79 & 7.70 & 7.69 & 7.70 & 7.85 \\
\hline Day 4 & 6.25 & 7.75 & 7.72 & 7.83 & 7.86 & 7.99 \\
\hline Day 5 & & 7.70 & 7.79 & 7.72 & 7.94 & 8.01 \\
\hline Day 6 & & 8.17 & 7.98 & 7.83 & 7.82 & 8.01 \\
\hline Final & 7.77 & 7.76 & 7.59 & 7.55 & 7.66 & 7.95 \\
\hline
\end{tabular}

Dissolved Oxygen (Before Renewal)

\begin{tabular}{|l|r|r|r|r|r|r|}
\hline \multicolumn{7}{|c|}{ Test Concentration } \\
\hline & Control & $6.25 \%$ & $12.5 \%$ & $25 \%$ & $50 \%$ & $100 \%$ \\
\hline Day 1 & & 8.4 & 8.4 & 8.3 & 8.2 & 8.2 \\
\hline Day 2 & 8.5 & 8.5 & 8.5 & 8.5 & 8.3 & 8.5 \\
\hline Day 3 & & 8.6 & 8.6 & 8.6 & 8.6 & 8.6 \\
\hline Day 4 & 8.9 & 8.4 & 8.6 & 8.6 & 8.7 & 8.8 \\
\hline Day 5 & & 8.6 & 8.5 & 8.5 & 8.6 & 8.6 \\
\hline Day 6 & & 8.4 & 8.4 & 8.6 & 8.6 & 8.9 \\
\hline Final & 8.6 & 8.4 & 8.4 & 8.5 & 8.5 & 8.5 \\
\hline
\end{tabular}

Test Results Reviewed and Approved By:

Temperature

\begin{tabular}{|l|r|}
\hline \multicolumn{2}{|c|}{ Incubator ${ }^{\circ} \mathrm{C}$} \\
\hline Initial & 25.0 \\
\hline Day 1 & 24.2 \\
\hline Day 2 & 24.9 \\
\hline Day 3 & 25.3 \\
\hline Day 4 & 24.5 \\
\hline Day 5 & 24.0 \\
\hline Day 6 & 24.0 \\
\hline Final & 24.5 \\
\hline
\end{tabular}




\title{
7 Day Chronic Definitive Survival and Reproduction Bioassay Method: EPA/600/4-91/002
}

\author{
Test Organism: $\quad$ Ceriodaphnia dubia
}

Facility: WSRC/ A-01

Sample ID: A-01 W/ Daphnia ambigua

Aiken County

ETT\#: 19113

Sample ID: January 22, 2002 


\section{CHRONIC DEFINITIVE SURVIVAL AND REPRODUCTION TEST Survival and Reproduction Results}

Client: $\quad$ WSRCI A-0I

Sample Identification:

Test Date:

A-01 W/Daphnia ambigua

January 22, 2002

\begin{tabular}{|c|c|c|c|c|c|c|}
\hline \multicolumn{7}{|c|}{ Reproduction at } \\
\hline Rep. & Day 3/4 & Day 5 & Day 6 & Day 7 & Day 8 & Total \\
\hline 1 & 4 & 8 & 0 & 15 & 0 & 27 \\
\hline 2 & 5 & 8 & 0 & 15 & 0 & 28 \\
\hline 3 & 0 & 2 & 6 & 0 & 7 & 15 \\
\hline 4 & 6 & 0 & 10 & 15 & 0 & 31 \\
\hline 5 & 5 & 7 & 0 & 13 & 0 & 25 \\
\hline 6 & 6 & 7 & 0 & 13 & 0 & 26 \\
\hline 7 & male & $\mathrm{L}$ & $\mathrm{L}$ & $\mathrm{L}$ & $\mathrm{L}$ & $\mathrm{L}$ \\
\hline 8 & 4 & 0 & 9 & 12 & 0 & 25 \\
\hline 9 & 6 & 6 & 0 & 13 & 0 & 25 \\
\hline I0 & 5 & 0 & 8 & 0 & 13 & 26 \\
\hline
\end{tabular}

\begin{tabular}{|c|c|c|c|c|c|c|}
\hline \multicolumn{1}{|c|}{ Reproduction at } & \multicolumn{3}{|c|}{$12.5 \%$} & \multicolumn{3}{c|}{ Concentration } \\
\hline Rep. & Day 3/4 & Day 5 & Day 6 & Day 7 & Day 8 & Total \\
\hline 1 & 5 & 0 & 10 & 0 & 13 & 28 \\
\hline 2 & 6 & 0 & 10 & 0 & 13 & 29 \\
\hline 3 & 4 & 0 & 8 & 11 & 0 & 23 \\
\hline 4 & 6 & 0 & 10 & 12 & 0 & 28 \\
\hline 5 & 0 & 0 & 7 & 12 & 0 & 19 \\
\hline 6 & 0 & 0 & 10 & 12 & 0 & 22 \\
\hline 7 & 4 & 0 & 6 & 0 & 11 & 21 \\
\hline 8 & 5 & 0 & 9 & 13 & 0 & 27 \\
\hline 9 & 5 & 0 & 8 & 0 & 10 & 23 \\
\hline 10 & 6 & 0 & 12 & 15 & 0 & 33 \\
\hline
\end{tabular}

\begin{tabular}{|c|c|c|c|c|c|c|}
\hline \multicolumn{1}{|c|}{ Reproduction at } & \multicolumn{1}{|c|}{$50 \%$} & \multicolumn{3}{c|}{ Concentration } \\
\hline Rep. & Day 3/4 & Day 5 & Day 6 & Day 7 & Day 8 & Total \\
\hline 1 & 4 & 0 & 9 & 0 & 10 & 23 \\
\hline 2 & 4 & 0 & 9 & 10 & 0 & 23 \\
\hline 3 & 4 & 0 & 10 & 12 & 0 & 26 \\
\hline 4 & 6 & 0 & 10 & 12 & 0 & 28 \\
\hline 5 & 6 & 0 & 8 & 14 & 0 & 28 \\
\hline 6 & 5 & 0 & 12 & 14 & 0 & 31 \\
\hline 7 & 5 & 0 & 8 & 13 & 0 & 26 \\
\hline 8 & 7 & 0 & 10 & 12 & 0 & 29 \\
\hline 9 & 5 & 0 & 9 & 0 & 12 & 26 \\
\hline 10 & 4 & 0 & 11 & $X$ & $X$ & 15 \\
\hline
\end{tabular}

County: NPDES\#:

ETT \#:

\begin{tabular}{|c|c|c|c|c|c|c|}
\hline \multicolumn{2}{|c|}{ Reproduction at } & \multicolumn{5}{c|}{$6.25 \%$} \\
\hline Rep. & Day 3/4 & Day 5 & Day 6 & Day 7 & Day 8 & Total \\
\hline 1 & 3 & 0 & 9 & 0 & 14 & 26 \\
\hline 2 & 4 & 0 & 0 & 0 & 11 & 15 \\
\hline 3 & 4 & 0 & 9 & 10 & 0 & 23 \\
\hline 4 & 2 & 0 & 12 & 10 & 0 & 24 \\
\hline 5 & 6 & 0 & 10 & 11 & 0 & 27 \\
\hline 6 & $X$ & $X$ & $X$ & $X$ & $X$ & 0 \\
\hline 7 & $X$ & $X$ & $X$ & $X$ & $X$ & 0 \\
\hline 8 & 4 & 0 & 8 & 6 & 0 & 18 \\
\hline 9 & 3 & 0 & 0 & 0 & 8 & 11 \\
\hline 10 & 6 & 0 & 6 & 12 & 0 & 24 \\
\hline
\end{tabular}

\begin{tabular}{|c|c|c|c|c|c|c|}
\hline \multicolumn{2}{|c|}{ Reproduction at } & \multicolumn{4}{|c|}{ Concentration } \\
\hline Rep. & Day 3/4 & Day 5 & Day 6 & Day 7 & Day 8 & Total \\
\hline 1 & 6 & 0 & 7 & 0 & 12 & 25 \\
\hline 2 & 4 & 0 & 10 & 0 & 11 & 25 \\
\hline 3 & 0 & 0 & 10 & 10 & 0 & 20 \\
\hline 4 & 5 & 0 & 10 & 12 & 0 & 27 \\
\hline 5 & 0 & 0 & 11 & 12 & $X$ & 23 \\
\hline 6 & 5 & 0 & 12 & 13 & 0 & 30 \\
\hline 7 & 6 & 0 & 12 & 13 & 0 & 31 \\
\hline 8 & 5 & 0 & 12 & 13 & 0 & 30 \\
\hline 9 & 6 & 0 & 10 & 0 & 11 & 27 \\
\hline 10 & 5 & 0 & 13 & 10 & 0 & 28 \\
\hline
\end{tabular}

\begin{tabular}{|c|c|c|c|c|c|c|}
\hline \multicolumn{2}{|c|}{ Reproduction at } & \multicolumn{6}{c|}{ 100\% } \\
\hline Rep. & Day 3/4 & Day 5 & Day 6 & Day 7 & Day 8 & Tocal \\
\hline 1 & 6 & 0 & 6 & 8 & 0 & 20 \\
\hline 2 & 6 & 0 & 8 & 0 & 12 & 26 \\
\hline 3 & 4 & 0 & 7 & 9 & 0 & 20 \\
\hline 4 & 5 & 0 & 9 & 12 & 0 & 26 \\
\hline 5 & 6 & 0 & 10 & 14 & 0 & 30 \\
\hline 6 & 5 & 0 & 12 & 14 & 0 & 31 \\
\hline 7 & 7 & 0 & 10 & 15 & 0 & 32 \\
\hline 8 & 6 & 0 & 9 & 12 & 0 & 27 \\
\hline 9 & 5 & 0 & 9 & 0 & 10 & 24 \\
\hline 10 & 6 & 0 & 10 & 13 & 0 & 29 \\
\hline
\end{tabular}




\section{CHRONIC DEFINITIVE SURVIVAL AND REPRODUCTION TEST \\ Statistical Analyses}

Client:

Sample Identification:

Test Date:

\section{WSRCI A-01}

A-01 W/ Daphnia ambigua

January 22, 2002

\section{Test for Normality}

Test Used: Kolmogorov D

$$
\begin{array}{ll}
D= & N A \\
\text { critical } & \text { NA }
\end{array}
$$

NA
Test for Homogeneity of Variance

Test Used: Bartlett's Test

$B=N A$
critical $N A$

\begin{tabular}{|c|c|c|c|c|c|c|}
\hline \multicolumn{2}{|l|}{ Text Type Used: } & \multicolumn{5}{|c|}{$\begin{array}{l}\text { Non-Linear Regression - Logistic Model } \\
\text { Calculated Using MMP Software } \\
\text { \% Effluent }\end{array}$} \\
\hline Errect & MHSF & $6.3 \%$ & $125 \%$ & $25.0 \%$ & $50.0 \%$ & $100 \%$ \\
\hline Survival & $100 x$ & $100 \%$ & $100 \%$ & $90 \%$ & $90 \%$ & $100 \%$ \\
\hline Prodicted & $100 \%$ & $100 \%$ & $100 \%$ & $100 \%$ & $100 \%$ & $100 \%$ \\
\hline Reproduction & 25.3 & 21.0 & 25.3 & 26.6 & 25.5 & 26.5 \\
\hline Prodictod & 243 & 24.3 & 24.3 & 24.3 & 24.3 & 24.3 \\
\hline Verianoe & 18.75 & 32.57 & 18.90 & 11.82 & 19.83 & 17.83 \\
\hline Teat Parameler & $17 \%$ & & & & MSD = & 4.68 \\
\hline & alpha & beta & gamina & \multicolumn{2}{|c|}{$\begin{array}{l}\text { \% Reduction } \\
\text { CTC- } 93.4 \%\end{array}$} & Pasa/rall \\
\hline Survival & 3.00 & -200.00 & 0.00 & $0.0 \%$ & & Pass \\
\hline \multirow[t]{2}{*}{ Reproduction } & 26.00 & -196.00 & 24.30 & $0.0 \%$ & & Pass \\
\hline & $\begin{array}{l}1 C 25= \\
\text { NOEC }=\end{array}$ & $\begin{array}{l}>100 \% \\
>100 \%\end{array}$ & & & & \\
\hline
\end{tabular}

NA

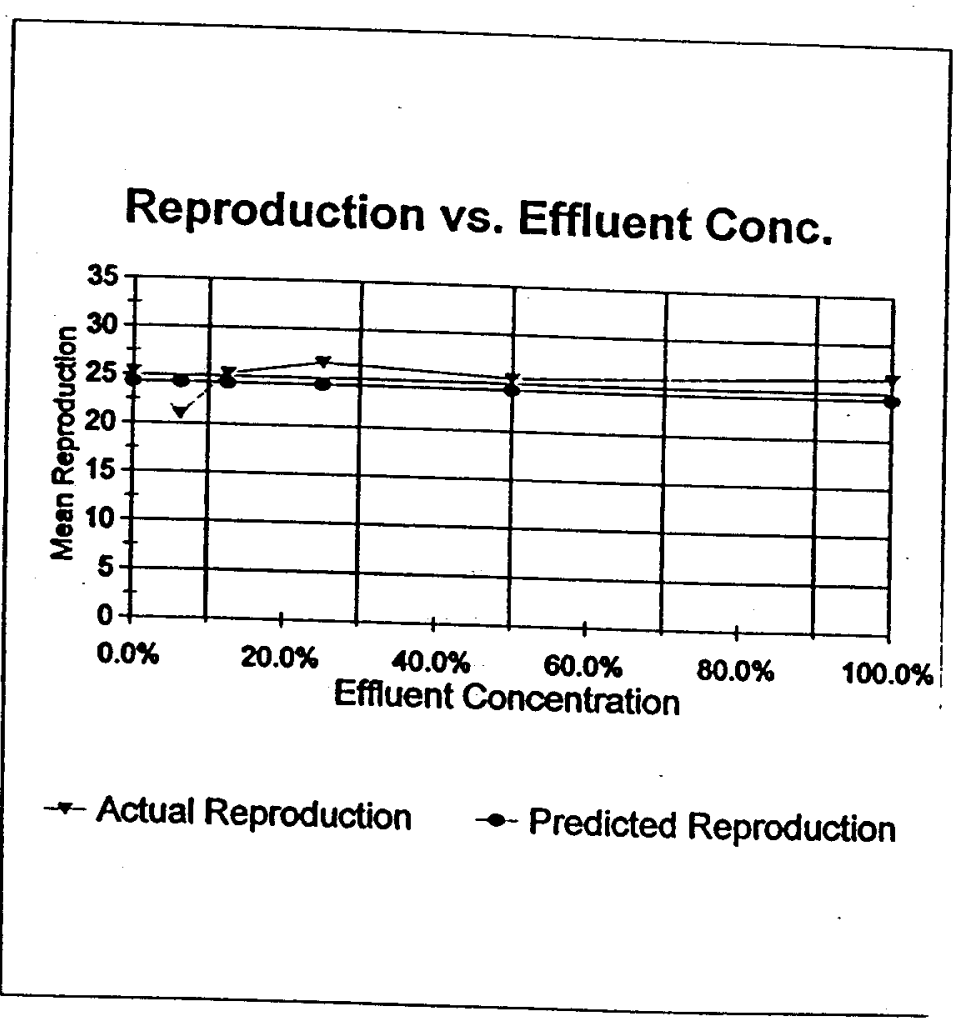

Summary

The reductions in survival and reproduction at the CTC are $<25 \%$ - Pass 


\section{CHRONIC DEFINITIVE SURVIVAL AND REPRODUCTION TEST \\ Water Chemistry}

Client:

WSRCI A-01

Sample Identification:

Test Date:

A-01 W/ Daphnia ambigua

January 22, 2002

pH (After Renewal)

\begin{tabular}{|l|r|r|r|r|r|r|}
\hline \multicolumn{7}{|c|}{ Test Concentration } \\
\hline & Control & $6.25 \%$ & $12.5 \%$ & $25.0 \%$ & $50.0 \%$ & $100 \%$ \\
\hline Initial & 7.64 & 7.72 & 7.77 & 7.47 & 7.21 & 7.89 \\
\hline Day 1 & 7.10 & 7.37 & 7.36 & 7.71 & 7.20 & \\
\hline Day 2 & 7.06 & 6.97 & 7.08 & 7.08 & 7.09 & 7.08 \\
\hline Day 3 & 7.08 & 7.51 & 7.80 & 7.44 & 7.47 & 7.46 \\
\hline Day 4 & 7.57 & 7.61 & 7.57 & 7.53 & 7.55 & 7.54 \\
\hline Day 5 & 7.26 & 8.29 & 8.16 & 7.98 & 7.85 & 7.61 \\
\hline Day 6 & 7.25 & 7.48 & 7.11 & 7.02 & 6.93 & 7.01 \\
\hline
\end{tabular}

Dissolved Oxygen (After Renewal)

\begin{tabular}{|l|r|r|r|r|r|r|}
\hline & \multicolumn{7}{|c|}{ Test Concentration } \\
\hline Initial & 8.4 & $6.25 \%$ & $12.5 \%$ & $25.0 \%$ & $50.0 \%$ & $100 \%$ \\
\hline Day 1 & 8.0 & 8.2 & 8.3 & 8.1 & 8.4 & 8.4 \\
\hline Day 2 & 8.2 & 8.3 & 8.3 & 8.2 & 8.2 & 8.2 \\
\hline Day 3 & 8.2 & 8.3 & 8.4 & 8.4 & 8.3 & 8.3 \\
\hline Day 4 & 8.0 & 8.3 & 8.3 & 8.4 & 8.4 & 8.4 \\
\hline Day 5 & & 8.3 & 8.3 & 8.4 & 8.4 & 8.4 \\
\hline Day 6 & 8.3 & 8.3 & 8.3 & 8.3 & 8.3 & 8.5 \\
\hline
\end{tabular}

Test Results Reviewed and Approved By:

pH (Before Renewal)
\begin{tabular}{|l|r|r|r|r|r|r|}
\hline \multicolumn{7}{|c|}{ Test Concentration } \\
\hline & Control & $6.25 \%$ & $12.5 \%$ & $25.0 \%$ & $50.0 \%$ & $100 \%$ \\
\hline Day 1 & 7.31 & 7.75 & 7.85 & 8.24 & 7.65 & \\
\hline Day 2 & 7.40 & 7.17 & 7.20 & 7.24 & 7.29 & 7.42 \\
\hline Day 3 & 7.17 & 7.40 & 7.40 & 7.41 & 7.41 & 7.42 \\
\hline Day 4 & 7.74 & 7.48 & 7.47 & 7.48 & 7.49 & 7.50 \\
\hline Day 5 & 7.74 & 8.86 & 8.64 & 8.46 & 8.31 & 8.11 \\
\hline Day 6 & 7.50 & 7.63 & 7.49 & 7.44 & 7.44 & 7.43 \\
\hline Final & 7.33 & 7.25 & 7.67 & 7.59 & 7.43 & 7.43 \\
\hline
\end{tabular}

Dissolved Oxygen (Before Renewal)

\begin{tabular}{|l|r|r|r|r|r|r|}
\hline \multicolumn{7}{|c|}{ Test Concentration } \\
\hline & \multicolumn{1}{|c|}{ Control } & $6.25 \%$ & $12.5 \%$ & $25.0 \%$ & $50.0 \%$ & $100 \%$ \\
\hline Day 1 & 8.0 & 8.2 & 8.3 & 8.3 & 8.3 & 8.3 \\
\hline Day 2 & 8.2 & 8.3 & 8.3 & 8.3 & 8.3 & 8.2 \\
\hline Day 3 & 8.1 & 8.2 & 8.2 & 8.2 & 8.1 & 8.1 \\
\hline Day 4 & 8.3 & 8.2 & 8.2 & 8.2 & 8.2 & 8.2 \\
\hline Day 5 & & 8.4 & 8.4 & 8.4 & 8.4 & 8.5 \\
\hline Day 6 & 8.3 & 8.3 & 8.3 & 8.3 & 8.2 & 8.2 \\
\hline Final & 8.0 & 8.4 & 8.3 & 8.4 & 8.4 & 8.4 \\
\hline
\end{tabular}

Temperature

\begin{tabular}{|l|r|}
\hline \multicolumn{1}{|c|}{ Incubator ${ }^{\circ} \mathrm{C}$} \\
\hline Initial & 25.2 \\
\hline Day 1 & 25.2 \\
\hline Day 2 & 25.1 \\
\hline Day 3 & 25.7 \\
\hline Day 4 & 24.7 \\
\hline Day 5 & 25.4 \\
\hline Day 6 & 25.2 \\
\hline Final & 25.6 \\
\hline
\end{tabular}




\title{
7 Day Chronic Definitive Survival and Reproduction Bioassay \\ Method: EPA/600/4-91/002
}

\author{
Test Organism: \\ Ceriodaphnia dubia
}

Facility: WSRC/A-11

Sample ID: A-11 W/ Daphnia ambigua

Aiken County

ETT\#: 19114

Sample ID: January 22, 2002 


\section{CHRONIC DEFINITIVE SURVIVAL AND REPRODUCTION TEST}

Survival and Reproduction Results

Client:

Sample Identification:

Test Date:

WSRC/A-1I

January 22, 2002

\begin{tabular}{|c|c|c|c|c|c|c|}
\hline \multicolumn{7}{|c|}{ Reproduction at } \\
\hline Rep. & Day 3/4 & Day 5 & Day 6 & Day 7 & Day 8 & Tocal \\
\hline 1 & 4 & 8 & 0 & 15 & 0 & 27 \\
\hline 2 & 5 & 8 & 0 & 15 & 0 & 28 \\
\hline 3 & 0 & 2 & 6 & 0 & 7 & 15 \\
\hline 4 & 6 & 0 & 10 & 15 & 0 & 31 \\
\hline 5 & 5 & 7 & 0 & 13 & 0 & 25 \\
\hline 6 & 6 & 7 & 0 & 13 & 0 & 26 \\
\hline 7 & male & L & L & L & L & L \\
\hline 8 & 4 & 0 & 9 & 12 & 0 & 25 \\
\hline 9 & 6 & 6 & 0 & 13 & 0 & 25 \\
\hline 10 & 5 & 0 & 8 & 0 & 13 & 26 \\
\hline
\end{tabular}

Reproduction at

\begin{tabular}{|c|c|c|c|c|c|c|}
\hline Reproduction at & \multicolumn{2}{|c|}{$12.5 \%$} & \multicolumn{3}{c|}{ Concentration } \\
\hline Rep. & Day 3/4 & Day 5 & Day 6 & Day 7 & Day 8 & Total \\
\hline 1 & 0 & 5 & 9 & 0 & 13 & 27 \\
\hline 2 & 0 & 4 & 3 & 0 & 13 & 20 \\
\hline 3 & $X$ & $X$ & $X$ & $X$ & $X$ & 0 \\
\hline 4 & 4 & 0 & 10 & 13 & 0 & 27 \\
\hline 5 & 3 & 0 & 10 & 13 & 0 & 26 \\
\hline 6 & 4 & 0 & 4 & 10 & 0 & 18 \\
\hline 7 & 3 & 0 & 9 & 13 & 0 & 25 \\
\hline 8 & 4 & 0 & 12 & 15 & 0 & 31 \\
\hline 9 & 0 & 4 & 9 & 0 & 12 & 25 \\
\hline 10 & 4 & 0 & 11 & 15 & 0 & 30 \\
\hline
\end{tabular}

\begin{tabular}{|c|c|c|c|c|c|c|}
\hline \multicolumn{3}{|c|}{ Reproduction at } & \multicolumn{2}{|c|}{$50 \%$} & \multicolumn{2}{|c|}{ Concentration } \\
\hline Rep. & Day 3/4 & Day 5 & Day 6 & Day 7 & Day : & Total \\
\hline 1 & 0 & 5 & 2 & 0 & 5 & 12 \\
\hline 2 & male & L & $L$ & $L$ & $L$ & $\mathbf{L}$ \\
\hline 3 & 4 & 0 & 10 & 13 & 0 & 27 \\
\hline 4 & 4 & 0 & 11 & 12 & 0 & 27 \\
\hline 5 & 0 & 0 & 3 & 0 & 0 & 3 \\
\hline 6 & 4 & 0 & 11 & 14 & 0 & 29 \\
\hline 7 & 3 & 2 & 10 & 0 & 0 & 15 \\
\hline 8 & 3 & 0 & 12 & 14 & 0 & 29 \\
\hline 9 & 0 & 5 & 9 & 0 & 15 & 29 \\
\hline 10 & 0 & 4 & 10 & $x$ & $x$ & 14 \\
\hline
\end{tabular}

County:

NPDES\#:

ETT \#:
Aiken

SC

19114

\begin{tabular}{|c|c|c|c|c|c|c|}
\hline \multicolumn{2}{|c|}{ Reproduction at } & \multicolumn{2}{c|}{$6.25 \%$} & \multicolumn{2}{c|}{ Concentration } \\
\hline Rep. & Day 3/4 & Day 5 & Day 6 & Day 7 & Day 8 & Total \\
\hline 1 & 0 & 5 & 9 & 0 & 13 & 27 \\
\hline 2 & 0 & 5 & 10 & 0 & 15 & 30 \\
\hline 3 & 4 & 0 & 7 & 12 & 0 & 23 \\
\hline 4 & 5 & 0 & 10 & 10 & 0 & 25 \\
\hline 5 & 2 & 0 & 8 & 10 & 0 & 20 \\
\hline 6 & 0 & 0 & 8 & 13 & 0 & 21 \\
\hline 7 & 0 & $X$ & $X$ & $X$ & $X$ & 0 \\
\hline 8 & 5 & 0 & 10 & 13 & 0 & 28 \\
\hline 9 & 0 & 5 & 9 & $X$ & $X$ & 14 \\
\hline 10 & 4 & 0 & 12 & 15 & 0 & 31 \\
\hline
\end{tabular}

\begin{tabular}{|c|c|c|c|c|c|c|}
\hline \multicolumn{1}{|c|}{ Reproduction at } & \multicolumn{3}{|c|}{$25 \%$} & Concentration \\
\hline Rep. & Day 3/4 & Day 5 & Day 6 & Day 7 & Day 8 & Total \\
\hline 1 & 0 & 5 & 10 & 0 & 9 & 24 \\
\hline 2 & 0 & 0 & 0 & 0 & 0 & 0 \\
\hline 3 & 0 & 5 & 9 & 0 & 10 & 24 \\
\hline 4 & 5 & 0 & 8 & $X$ & $X$ & 13 \\
\hline 5 & 0 & 4 & 11 & 0 & 13 & 28 \\
\hline 6 & 4 & 0 & 10 & 15 & 0 & 29 \\
\hline 7 & 4 & 0 & 12 & 14 & 0 & 30 \\
\hline 8 & 3 & 0 & 13 & 15 & 0 & 31 \\
\hline 9 & 0 & 5 & 11 & 0 & 12 & 28 \\
\hline 10 & 5 & 0 & 10 & 15 & 0 & 30 \\
\hline
\end{tabular}

\begin{tabular}{|c|c|c|c|c|c|c|}
\hline \multicolumn{2}{|c|}{ Reproduction at } & \multicolumn{1}{c|}{ 100\% } & \multicolumn{3}{c|}{ Concentration } \\
\hline Rep. & Day 3/4 & Day 5 & Day 6 & Day 7 & Day 8 & Tocal \\
\hline 1 & 0 & 2 & 0 & 9 & 0 & 11 \\
\hline 2 & 4 & 0 & 11 & 13 & 0 & 28 \\
\hline 3 & 4 & 0 & 5 & 11 & 0 & 20 \\
\hline 4 & 4 & 0 & 8 & 0 & 0 & 12 \\
\hline 5 & 4 & 5 & 4 & 12 & 0 & 25 \\
\hline 6 & 0 & 0 & 10 & 13 & 2 & 25 \\
\hline 7 & 5 & 0 & 7 & 12 & 0 & 24 \\
\hline 8 & 4 & 0 & 8 & 13 & 0 & 25 \\
\hline 9 & 4 & 0 & 10 & 13 & 0 & 27 \\
\hline 10 & 3 & 0 & 9 & 14 & 0 & 26 \\
\hline
\end{tabular}




\section{CHRONIC DEFINITIVE SURVIVAL AND REPRODUCTION TEST \\ Statistical Analyses}

Client:

Sample Identification:

Test Date:

\section{WSRC/A-11}

A-1I W/ Daphnia ambigua

January 22, 2002

\begin{tabular}{|cll|}
\hline Test for Normality & & \\
Test Used: Kolmogorov D & D = & NA \\
& critical & NA \\
NA & & \\
& & \\
& \\
\hline
\end{tabular}

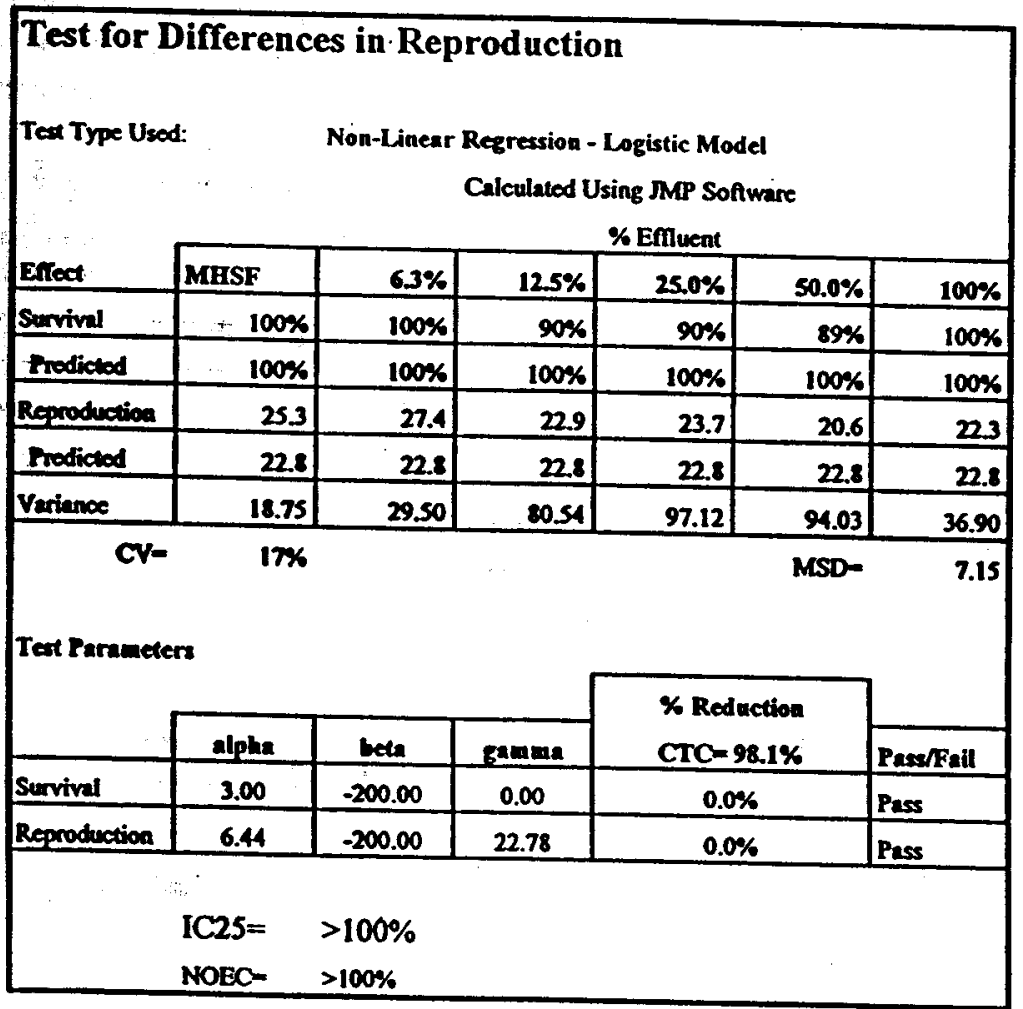

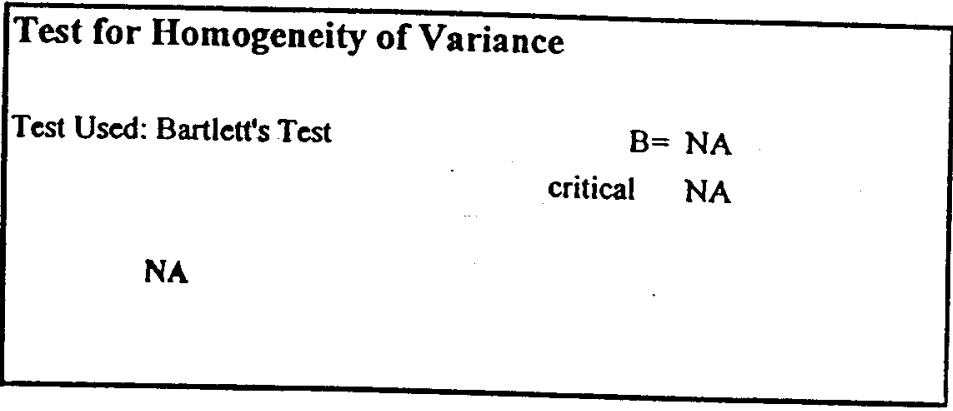

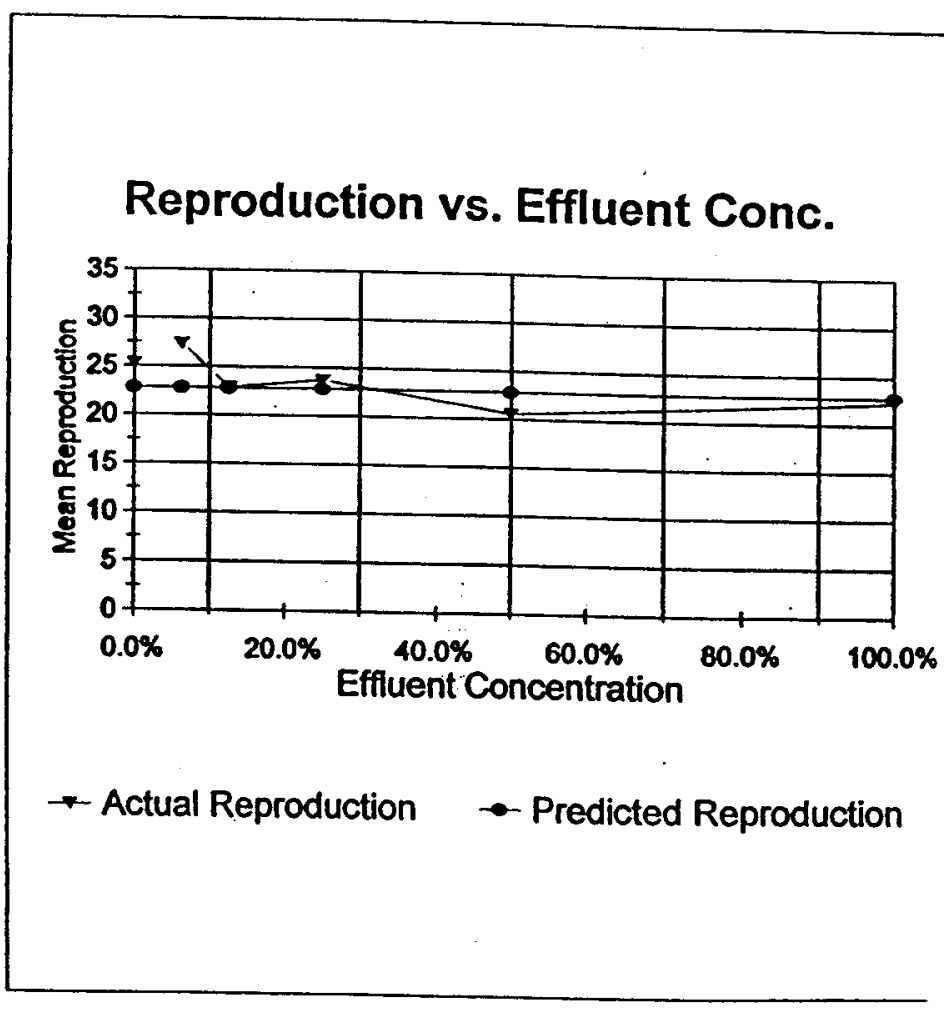

\section{Summary}

The reductions in survival and reproduction at the CTC are $<25 \%$ - Pass 


\section{CHRONIC DEFINITIVE SURVIVAL AND REPRODUCTION TEST Water Chemistry}

Client:

\section{WSRCIA-11}

Sample Identification:

Test Date:

A-11 W/ Daphnia ambigua

January 22, 2002

pH (After Renewal)

\begin{tabular}{|l|r|r|r|r|r|r|}
\hline \multicolumn{7}{|c|}{ Test Concentration } \\
\hline & Control & $6.25 \%$ & $12.5 \%$ & $25.0 \%$ & $50.0 \%$ & $100 \%$ \\
\hline Initial & 7.64 & 7.94 & 8.20 & 7.97 & 7.86 & 7.80 \\
\hline Day 1 & 7.10 & 7.08 & 7.11 & 7.13 & 7.17 & 7.21 \\
\hline Day 2 & 7.06 & 8.42 & 7.71 & 7.56 & 7.48 & 7.45 \\
\hline Day 3 & 7.08 & 7.36 & 3.34 & 3.32 & 7.30 & 7.31 \\
\hline Day 4 & 7.57 & 7.82 & 7.72 & 7.58 & 7.51 & 7.45 \\
\hline Day 5 & 7.26 & 7.66 & 7.59 & 7.53 & 7.45 & 7.43 \\
\hline Day 6 & 7.25 & 7.00 & 6.95 & 6.89 & 6.84 & 6.92 \\
\hline
\end{tabular}

Dissolved Oxygen (After Renewal)

\begin{tabular}{|l|r|r|r|r|r|r|}
\hline & \multicolumn{7}{|c|}{ Test Concentration } \\
\hline Initial & 8.4 & 8.3 & 8.4 & 8.4 & 8.3 & 8.4 \\
\hline Day 1 & 8.0 & 8.4 & 8.4 & 8.4 & 8.4 & 8.4 \\
\hline Day 2 & 8.2 & 8.4 & 8.4 & 8.4 & 8.4 & 8.4 \\
\hline Day 3 & 8.2 & & & & & \\
\hline Day 4 & 8.0 & 8.4 & 8.4 & 8.4 & 8.5 & 8.5 \\
\hline Day 5 & & 8.3 & 8.3 & 8.4 & 8.5 & 8.6 \\
\hline Day 6 & 8.3 & 8.4 & 8.4 & 8.4 & 8.4 & 8.4 \\
\hline
\end{tabular}

pH (Before Renewal)

\begin{tabular}{|l|r|r|r|r|r|r|}
\hline & Control & $6.25 \%$ & $12.5 \%$ & $25.0 \%$ & $50.0 \%$ & $100 \%$ \\
\hline Day 1 & 7.31 & 7.03 & 7.08 & 7.11 & 7.17 & 7.26 \\
\hline Day 2 & 7.40 & 8.47 & 8.00 & 7.90 & 7.81 & 7.70 \\
\hline Day 3 & 7.17 & 7.36 & 7.35 & 7.37 & 7.38 & 7.38 \\
\hline Day 4 & 7.74 & 8.10 & 7.99 & 7.93 & 7.81 & 7.78 \\
\hline Day 5 & 7.74 & 7.82 & 7.80 & 7.80 & 7.80 & 7.60 \\
\hline Day 6 & 7.50 & 7.41 & 7.37 & 7.34 & 7.31 & 7.33 \\
\hline Final & 7.33 & 7.82 & 7.75 & 7.63 & 7.54 & \\
\hline
\end{tabular}

Dissolved Oxygen (Before Renewal)

\begin{tabular}{|l|r|r|r|r|r|r|}
\hline & Control & $6.25 \%$ & $12.5 \%$ & $25.0 \%$ & $50.0 \%$ & $100 \%$ \\
\hline Day 1 & 8.0 & & & & & 8.3 \\
\hline Day 2 & 8.2 & 8.5 & 8.5 & 8.5 & 8.4 & 8.5 \\
\hline Day 3 & 8.1 & 8.4 & 8.4 & 8.4 & 8.3 & 8.3 \\
\hline Day 4 & 8.3 & 8.4 & 8.4 & 8.3 & 8.5 & 8.5 \\
\hline Day 5 & & 3.2 & 8.3 & 8.4 & 8.4 & 8.4 \\
\hline Day 6 & 8.3 & 8.3 & 8.3 & 8.3 & 8.3 & 8.3 \\
\hline Final & 8.0 & 8.4 & 8.4 & 8.5 & 8.3 & 8.5 \\
\hline
\end{tabular}

Test Results Reviewed and Approved By:

Temperature
\begin{tabular}{|l|r|}
\hline \multicolumn{1}{|c|}{ Incubator ${ }^{\circ} \mathrm{C}$} \\
\hline Initial & 25.2 \\
\hline Day 1 & 25.2 \\
\hline Day 2 & 25.1 \\
\hline Day 3 & 25.7 \\
\hline Day 4 & 24.7 \\
\hline Day 5 & 25.4 \\
\hline Day 6 & 25.2 \\
\hline Final & 25.6 \\
\hline
\end{tabular}




\section{Day Chronic Definitive Survival and Reproduction Bioassay \\ Method: EPA/600/4-91/002}

Test Organism: $\quad$ Ceriodaphnia dubia

Facility: WSRC/G-10

Sample ID: G-10 W/ Daphnia ambigua

Aiken County

ETT\#: 19115

Sample ID: January 22, 2002 


\section{CHRONIC DEFINITIVE SURVIVAL AND REPRODUCTION TEST \\ Survival and Reproduction Results}

Client:

Sample Identification:

Test Date:

WSRC/G-10

January 22, 2002

\begin{tabular}{|c|c|c|c|c|c|c|}
\hline \multicolumn{2}{|c|}{ Reproduction at } & \multicolumn{7}{c|}{ MHSF } & Control \\
\hline Rep. & Day 3/4 & Day 5 & Day 6 & Day 7 & Day 8 & Total \\
\hline 1 & 4 & 8 & 0 & 15 & 0 & 27 \\
\hline 2 & 5 & 8 & 0 & 15 & 0 & 28 \\
\hline 3 & 0 & 2 & 6 & 0 & 7 & 15 \\
\hline 4 & 6 & 0 & 10 & 15 & 0 & 31 \\
\hline 5 & 5 & 7 & 0 & 13 & 0 & 25 \\
\hline 6 & 6 & 7 & 0 & 13 & 0 & 26 \\
\hline 7 & male & L & L & L & L & L \\
\hline 8 & 4 & 0 & 9 & 12 & 0 & 25 \\
\hline 9 & 6 & 6 & 0 & 13 & 0 & 25 \\
\hline I0 & 5 & 0 & 8 & 0 & 13 & 26 \\
\hline
\end{tabular}

\begin{tabular}{|c|c|c|c|c|c|c|}
\hline Reproduction at & \multicolumn{6}{|c|}{$12.5 \%$} \\
\hline Rep. & Day 3/4 & Day 5 & Day 6 & Day 7 & Day 8 & Toual \\
\hline 1 & 7 & 6 & 0 & 13 & 0 & 26 \\
\hline 2 & 5 & 0 & 8 & 12 & 0 & 25 \\
\hline 3 & 4 & 1 & 8 & 10 & 0 & 23 \\
\hline 4 & 6 & 0 & 14 & 15 & 0 & 35 \\
\hline 5 & 4 & 0 & 10 & 13 & 0 & 27 \\
\hline 6 & 5 & 0 & 10 & 13 & 0 & 28 \\
\hline 7 & 6 & 0 & 10 & 12 & 0 & 28 \\
\hline 8 & 6 & 0 & 13 & 13 & 0 & 32 \\
\hline 9 & 5 & 0 & 10 & 12 & 0 & 27 \\
\hline 10 & 6 & 0 & 8 & 12 & 0 & 26 \\
\hline
\end{tabular}

\begin{tabular}{|c|c|c|c|c|c|c|}
\hline Reproduction at & \multicolumn{6}{|c|}{ Concentration } \\
\hline Rep. & Day 3/4 & Day 5 & Day 6 & Day 7 & Day 8 & Toul \\
\hline 1 & 8 & 7 & $X$ & $X$ & $X$ & 15 \\
\hline 2 & 4 & 0 & 7 & 10 & 0 & 21 \\
\hline 3 & 4 & 0 & 9 & 12 & 0 & 25 \\
\hline 4 & 6 & 0 & 7 & 13 & 0 & 26 \\
\hline 5 & 5 & 0 & 8 & 13 & 0 & 26 \\
\hline 6 & 7 & 0 & 10 & 12 & 0 & 29 \\
\hline 7 & 6 & 1 & 12 & 13 & 0 & 32 \\
\hline 8 & 4 & 0 & 9 & 0 & 0 & 13 \\
\hline 9 & 5 & 0 & 13 & 13 & 0 & 31 \\
\hline 10 & 3 & 0 & 7 & 14 & 0 & 24 \\
\hline
\end{tabular}

$\begin{array}{ll}\text { County: } & \text { Aiken } \\ \text { NPDES\#: } & \text { SC } \\ \text { ETT \#: } & 19115\end{array}$

\begin{tabular}{|c|c|c|c|c|c|c|}
\hline Reproduction at & \multicolumn{6}{|c|}{$6.25 \%$} \\
\hline Rep. & Day 3/4 & Day 5 & Day 6 & Day 7 & Day 8 & Total \\
\hline 1 & 4 & 8 & 0 & 13 & 0 & 25 \\
\hline 2 & 5 & 0 & 6 & 0 & 13 & 24 \\
\hline 3 & 6 & 0 & 8 & 0 & 0 & 14 \\
\hline 4 & 4 & 0 & 9 & 10 & 0 & 23 \\
\hline 5 & 3 & 0 & 9 & 11 & 0 & 23 \\
\hline 6 & 5 & 0 & 13 & 13 & 0 & 31 \\
\hline 7 & 6 & 0 & 9 & 12 & 0 & 27 \\
\hline 8 & 5 & 0 & 10 & 13 & 0 & 28 \\
\hline 9 & 0 & 0 & 0 & $X$ & $X$ & 0 \\
\hline 10 & 4 & 10 & 0 & 0 & 0 & 14 \\
\hline
\end{tabular}

\begin{tabular}{|c|c|c|c|c|c|c|}
\hline \multicolumn{2}{|c|}{ Reproduction at } & \multicolumn{3}{c|}{ Concentration } \\
\hline Rep. & Day 3/4 & Day 5 & Day 6 & Day 7 & Day 8 & Total \\
\hline 1 & 3 & 6 & 0 & 14 & 0 & 23 \\
\hline 2 & 4 & 0 & 12 & 12 & 0 & 28 \\
\hline 3 & 6 & 0 & 7 & 11 & 0 & 24 \\
\hline 4 & 5 & 0 & 11 & 12 & 0 & 28 \\
\hline 5 & 5 & 0 & 11 & 13 & 0 & 29 \\
\hline 6 & 5 & 0 & 12 & 9 & 0 & 26 \\
\hline 7 & 6 & 0 & 16 & 12 & 0 & 34 \\
\hline 8 & 5 & 0 & $X$ & $X$ & $X$ & 5 \\
\hline 9 & 5 & 0 & 17 & 13 & 0 & 35 \\
\hline 10 & 4 & 0 & 10 & 11 & 0 & 25 \\
\hline
\end{tabular}

\begin{tabular}{|c|c|c|c|c|c|c|}
\hline Reproduction at & \multicolumn{5}{c|}{$100 \%$} & Concentration \\
\hline Rep. & Day 3/4 & Day 5 & Day 6 & Day 7 & Day 8 & Toial \\
\hline 1 & 5 & 0 & 4 & 11 & 0 & 20 \\
\hline 2 & 3 & 5 & 0 & 12 & 0 & 20 \\
\hline 3 & 6 & 0 & 8 & 11 & 0 & 25 \\
\hline 4 & 7 & 0 & 7 & 12 & 0 & 26 \\
\hline 5 & 4 & 0 & 10 & 12 & 0 & 26 \\
\hline 6 & 4 & 1 & 8 & 12 & 0 & 25 \\
\hline 7 & 6 & 0 & 10 & 12 & 0 & 28 \\
\hline 8 & 5 & 0 & 12 & 11 & 0 & 28 \\
\hline 9 & 4 & 0 & 11 & 16 & 0 & 31 \\
\hline 10 & 6 & 0 & 11 & 13 & 0 & 30 \\
\hline
\end{tabular}




\section{CHRONIC DEFINITIVE SURVIVAL AND REPRODUCTION TEST \\ Statistical Analyses}

Client: $\quad$ WSRC/G-10

Sample Identification: $\quad$ G-10 W/ Daphnia ambigua

Test Date: January 22, 2002

\section{Test for Normality}

Test Used: Kolmogorov D

$\begin{array}{ll}D= & \text { NA } \\ \text { critical } & \text { NA }\end{array}$

NA
Test for Homogeneity of Variance

Test Used: Bartlett's Test

$B=N A$

critical NA

NA

\begin{tabular}{|c|c|c|c|c|c|c|}
\hline \multicolumn{7}{|c|}{ Test for Differences in Reproduction } \\
\hline \multicolumn{7}{|c|}{ Non-Linear Regression - Logistic Modd } \\
\hline Eriod & MHSF & $6.3 \%$ & $12.5 \%$ & $25.0 \%$ & $50.0 \%$ & $100 \%$ \\
\hline sumived & $100 \%$ & $100 \%$ & $100 \%$ & $90 \%$ & $90 \%$ & $100 \%$ \\
\hline Prodictod & $.100 x$ & $100 \%$ & $100 \%$ & $100 \%$ & $100 \%$ & $100 \%$ \\
\hline Reproduction & 25.3 & 23.2 & 27.7 & 25.7 & 24.2 & 25.9 \\
\hline Prodicald & 24.9 & 24.9 & 24.9 & 24.9 & 24.9 & 24.9 \\
\hline Varianoce & 18.75 & 33.94 & 12.01 & 68.46 & 39.73 & 13.66 \\
\hline $\begin{array}{l}\text { CV- } \\
\text { Test Paramet }\end{array}$ & $17 \%$ & & & & MSD $=$ & 5.93 \\
\hline & alpha & beta & gamma & \multicolumn{2}{|c|}{$\begin{array}{l}\text { \% Roduction } \\
\text { CTC-19\% CTC-100\% }\end{array}$} & \begin{tabular}{|l} 
PeserFail \\
\end{tabular} \\
\hline Survival & 3.00 & -200.00 & 0.00 & $0.0 \%$ & $0.0 \%$ & Pass \\
\hline Reproduction & 4.78 & -200.00 & 24.93 & $0.0 \%$ & $0.0 \%$ & Pess \\
\hline & $\begin{array}{l}1 C 25= \\
\text { NOEC }=\end{array}$ & $\begin{array}{l}>100 \% \\
>100 \%\end{array}$ & & & & \\
\hline
\end{tabular}

Reproduction vs. Effluent Conc.

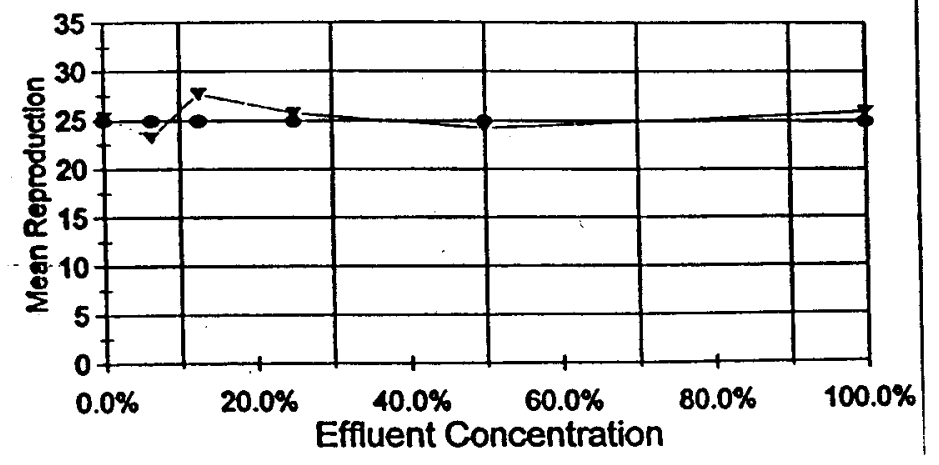

$\rightarrow$ Actual Reproduction $\rightarrow$ - Predicted Reproduction

Summary

The reductions in survival and reproduction at the CTC are $<25 \%-$ Pass 


\section{CHRONIC DEFINITIVE SURVIVAL AND REPRODUCTION TEST \\ Water Chemistry}

Client: WSRC/G-10

Sample Identification: G-10 W/ Daphnia ambigua

Test Date:

January 22, 2002

pH (After Renewal)
\begin{tabular}{|l|r|r|r|r|r|r|}
\hline \multicolumn{7}{|c|}{ Test Concentration } \\
\hline & Control & $6.25 \%$ & $12.5 \%$ & $25.0 \%$ & $50.0 \%$ & $100 \%$ \\
\hline Initial & 7.64 & 7.53 & 7.52 & 7.58 & 7.78 & 7.94 \\
\hline Day 1 & 7.10 & 7.16 & 7.29 & 7.53 & 7.55 & 7.80 \\
\hline Day 2 & 7.06 & 7.10 & 7.14 & 7.30 & 7.68 & 7.81 \\
\hline Day 3 & 7.08 & 7.15 & 7.23 & 7.36 & 7.55 & 7.78 \\
\hline Day 4 & 7.57 & 7.63 & 7.45 & 7.63 & 7.82 & 8.14 \\
\hline Day 5 & 7.26 & 7.29 & 7.39 & 7.52 & 7.56 & \\
\hline Day 6 & 7.25 & 7.24 & 7.22 & 7.29 & 7.61 & 7.89 \\
\hline
\end{tabular}

pH (Before Renewal)

\begin{tabular}{|l|r|r|r|r|r|r|}
\hline \multicolumn{7}{|c|}{ Test Concentration } \\
\hline & Control & $6.25 \%$ & $12.5 \%$ & $25.0 \%$ & $50.0 \%$ & $100 \%$ \\
\hline Day 1 & 7.31 & 7.29 & 7.39 & 7.51 & 7.71 & 7.91 \\
\hline Day 2 & 7.40 & 7.32 & 7.30 & 7.36 & 7.67 & 7.78 \\
\hline Day 3 & 7.17 & 7.31 & 7.45 & 7.59 & 7.72 & 7.99 \\
\hline Day 4 & 7.74 & 7.88 & 7.55 & 7.62 & 7.75 & 8.05 \\
\hline Day 5 & 7.74 & 7.66 & 7.57 & 7.73 & 7.82 & \\
\hline Day 6 & 7.50 & 7.43 & 7.37 & 7.38 & 7.55 & 7.91 \\
\hline Final & 7.33 & 7.72 & 7.15 & 7.19 & 7.34 & 7.68 \\
\hline
\end{tabular}

Dissolved Oxygen (After Renewal)

\begin{tabular}{|l|r|r|r|r|r|r|}
\hline & Control & $6.25 \%$ & $12.5 \%$ & $25.0 \%$ & $50.0 \%$ & $100 \%$ \\
\hline Lnitial & 8.4 & 8.3 & 8.2 & 8.2 & 8.2 & 8.2 \\
\hline Day 1 & 8.0 & 8.0 & 8.0 & 8.1 & 8.1 & 8.2 \\
\hline Day 2 & 8.2 & 8.2 & 8.2 & 8.2 & 8.1 & 8.1 \\
\hline Day 3 & 8.2 & 8.2 & 8.1 & 8.1 & 8.1 & 8.1 \\
\hline Day 4 & 8.0 & 8.3 & 8.2 & 8.2 & 8.2 & 8.2 \\
\hline Day 5 & & 8.3 & 8.4 & 8.3 & 8.3 & 8.3 \\
\hline Day 6 & 8.3 & 8.3 & 8.3 & 8.3 & 8.2 & 8.1 \\
\hline
\end{tabular}

Dissolved Oxygen (Before Renewal)

\begin{tabular}{|l|r|r|r|r|r|r|}
\hline & \multicolumn{7}{|c|}{ Test Concentration } \\
\hline Day 1 & 8.0 & 8.0 & 8.0 & 7.9 & 7.9 & 7.7 \\
\hline Day 2 & 8.2 & 8.2 & 8.2 & 8.2 & 8.1 & 8.1 \\
\hline Day 3 & 8.1 & 8.1 & 8.1 & 8.0 & 8.0 & 8.0 \\
\hline Day 4 & 8.3 & 8.1 & 8.1 & 8.1 & 8.1 & 8.1 \\
\hline Day 5 & & 8.0 & 8.2 & 8.2 & 8.2 & 8.2 \\
\hline Day 6 & 8.3 & 8.3 & 8.2 & 8.2 & 8.1 & 8.0 \\
\hline Final & 8.0 & 8.1 & 8.2 & 8.3 & 8.3 & 8.3 \\
\hline
\end{tabular}

Temperature

\begin{tabular}{|l|r|}
\hline \multicolumn{2}{|c|}{ Incubator ${ }^{\circ} \mathrm{C}$} \\
\hline Initial & 25.2 \\
\hline Day 1 & 25.2 \\
\hline Day 2 & 25.1 \\
\hline Day 3 & 25.7 \\
\hline Day 4 & 24.7 \\
\hline Day 5 & 25.4 \\
\hline Day 6 & 25.2 \\
\hline Final & 25.6 \\
\hline
\end{tabular}

Test Results Reviewed and Approved By:

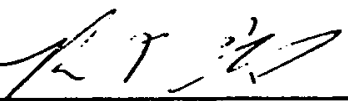




\section{Day Chronic Definitive Survival and Reproduction Bioassay \\ Method: EPA/600/4-91/002}

Test Organism:

Daphnia ambigua

Facility: WSRC

Sample ID: A-01

Aiken County

ETT\#: 19175

Sample ID: February 5, 2002 


\section{CHRONIC DEFINITIVE SURVIVAL AND REPRODUCTION TEST Survival and Reproduction Results}

Client:

WSRC

Sample Identification:

Test Date:

A-01

February 5, 2002

County:

NPDES\# :

ETT \#:

SC

19175

\begin{tabular}{|c|c|c|c|c|c|c|}
\hline \multicolumn{7}{|c|}{ Reproduction at } \\
\hline Rep. & Day 3/4 & Day 5 & Day 6 & Day 7 & Day 8 & Tolal \\
\hline 1 & 6 & 0 & 14 & 13 & 0 & 33 \\
\hline 2 & 4 & 9 & 16 & 0 & 0 & 29 \\
\hline 3 & 5 & 9 & 0 & 14 & 0 & 28 \\
\hline 4 & 4 & 11 & 10 & 0 & 0 & 25 \\
\hline 5 & 6 & 0 & 12 & 14 & 0 & 32 \\
\hline 6 & 6 & 0 & 13 & 14 & 0 & 33 \\
\hline 7 & 5 & 0 & 12 & 13 & 0 & 30 \\
\hline 8 & 6 & 0 & 11 & 15 & 0 & 32 \\
\hline 9 & 6 & 0 & 13 & 16 & 0 & 35 \\
\hline 10 & 4 & 0 & 11 & 13 & 0 & 28 \\
\hline
\end{tabular}

\begin{tabular}{|c|c|c|c|c|c|c|}
\hline Reproduction at & \multicolumn{6}{|c|}{$12.5 \%$} \\
\hline Rep. & Day 3/4 & Day 5 & Day 6 & Day 7 & Day 8 & Total \\
\hline 1 & 4 & $X$ & $X$ & $X$ & $X$ & 4 \\
\hline 2 & 5 & 11 & 15 & 0 & 0 & 31 \\
\hline 3 & 6 & 12 & 0 & 15 & 0 & 33 \\
\hline 4 & 5 & 12 & 15 & 0 & 0 & 32 \\
\hline 5 & $X$ & $X$ & $X$ & $X$ & $X$ & 0 \\
\hline 6 & 4 & 0 & 13 & 14 & 0 & 31 \\
\hline 7 & 6 & 0 & 10 & 15 & 0 & 31 \\
\hline 8 & 5 & 0 & 13 & 14 & 0 & 32 \\
\hline 9 & 5 & 0 & 10 & 12 & 0 & 27 \\
\hline 10 & 6 & 0 & 12 & 14 & 0 & 32 \\
\hline
\end{tabular}

\begin{tabular}{|c|c|c|c|c|c|c|}
\hline \multicolumn{2}{|c|}{ Reproduction at } \\
\hline Rep. & Day 3/4 & Day 5 & Day 6 & Day 7 & Day 8 & Toual \\
\hline 1 & 6 & 9 & 0 & 15 & 0 & 30 \\
\hline 2 & 1 & 12 & 13 & 0 & 0 & 26 \\
\hline 3 & 6 & 12 & 0 & 13 & 0 & 31 \\
\hline 4 & 4 & 12 & 10 & 0 & 0 & 26 \\
\hline 5 & 4 & 0 & 10 & $X$ & $X$ & 14 \\
\hline 6 & 6 & 0 & 13 & 14 & 0 & 33 \\
\hline 7 & 4 & 0 & 10 & 14 & 0 & 28 \\
\hline 8 & 5 & 1 & 12 & 16 & 0 & 34 \\
\hline 9 & 6 & 0 & 13 & 15 & 0 & 34 \\
\hline 10 & 6 & 0 & 14 & 14 & 0 & 34 \\
\hline
\end{tabular}

\begin{tabular}{|c|c|c|c|c|c|c|}
\hline \multicolumn{1}{|c|}{ Reproduction at } & \multicolumn{2}{|c|}{$25 \%$} & \multicolumn{3}{c|}{ Concentration } \\
\hline Rep. & Day 3/4 & Day 5 & Day 6 & Day 7 & Day 8 & Total \\
\hline 1 & 3 & 0 & 8 & 11 & 0 & 22 \\
\hline 2 & 6 & 9 & 12 & 0 & 0 & 27 \\
\hline 3 & 6 & 11 & $\times$ & $X$ & $\times$ & 17 \\
\hline 4 & 5 & 11 & 13 & 0 & 0 & 29 \\
\hline 5 & 5 & 0 & 11 & 10 & 0 & 26 \\
\hline 6 & 6 & 0 & 5 & 14 & 0 & 25 \\
\hline 7 & 6 & 0 & 11 & 13 & 0 & 30 \\
\hline 8 & 5 & 0 & 14 & 14 & 0 & 33 \\
\hline 9 & 5 & 0 & 12 & 15 & 0 & 32 \\
\hline 10 & 5 & 10 & 0 & 12 & 0 & 27 \\
\hline
\end{tabular}

\begin{tabular}{|c|c|c|c|c|c|c|}
\hline Reproduction at & \multicolumn{6}{|c|}{ Concentration } \\
\hline Rep. & Day 3/4 & Day 5 & Day 6 & Day 7 & Day 8 & Total \\
\hline 1 & 0 & 12 & 0 & 0 & 0 & 12 \\
\hline 2 & 3 & 11 & 11 & 0 & 0 & 25 \\
\hline 3 & 4 & 11 & 0 & 14 & 0 & 29 \\
\hline 4 & 5 & 13 & 15 & 0 & 0 & 33 \\
\hline 5 & 5 & 0 & 9 & 12 & 0 & 26 \\
\hline 6 & 5 & 0 & 13 & 17 & 0 & 35 \\
\hline 7 & 6 & 11 & 0 & 13 & 0 & 30 \\
\hline 8 & 6 & 1 & 12 & 11 & 0 & 30 \\
\hline 9 & 4 & 0 & 13 & 14 & 0 & 31 \\
\hline 10 & 5 & 0 & 11 & 15 & 0 & 31 \\
\hline
\end{tabular}

\begin{tabular}{|c|c|c|c|c|c|c|}
\hline \multicolumn{2}{|c|}{ Reproduction at } & \multicolumn{1}{c|}{$100 \%$} & \multicolumn{3}{c}{ Concentration } \\
\hline Rep. & Day 3/4 & Day 5 & Day 6 & Day 7 & Day 8 & Toul \\
\hline 1 & 5 & 11 & 0 & 15 & 0 & 31 \\
\hline 2 & 4 & 12 & 15 & 0 & 0 & 31 \\
\hline 3 & 3 & 12 & 0 & 14 & 0 & 29 \\
\hline 4 & 0 & 0 & 10 & 13 & 0 & 23 \\
\hline 5 & 6 & 9 & 0 & 14 & 0 & 29 \\
\hline 6 & 5 & 8 & 0 & 12 & 0 & 25 \\
\hline 7 & 2 & $X$ & $X$ & $X$ & $X$ & 2 \\
\hline 8 & 5 & 0 & 12 & 15 & 0 & 32 \\
\hline 9 & 4 & 11 & 0 & 17 & 0 & 32 \\
\hline 10 & 6 & 0 & 11 & 12 & 0 & 29 \\
\hline
\end{tabular}




\section{CHRONIC DEFINITIVE SURVIVAL AND REPRODUCTION TEST \\ Statistical Analyses}

Client:

WSRC

Sample Identification:

A-01

Test Date:

February 5, 2002

\section{Test for Normality}

Test Used: Kolmogorov D

$\begin{array}{ll}D= & \text { NA } \\ \text { critical } & \text { NA }\end{array}$

NA
Test for Homogeneity of Variance

Test Used: Bartlett's Test

$B=N A$

critical NA

NA

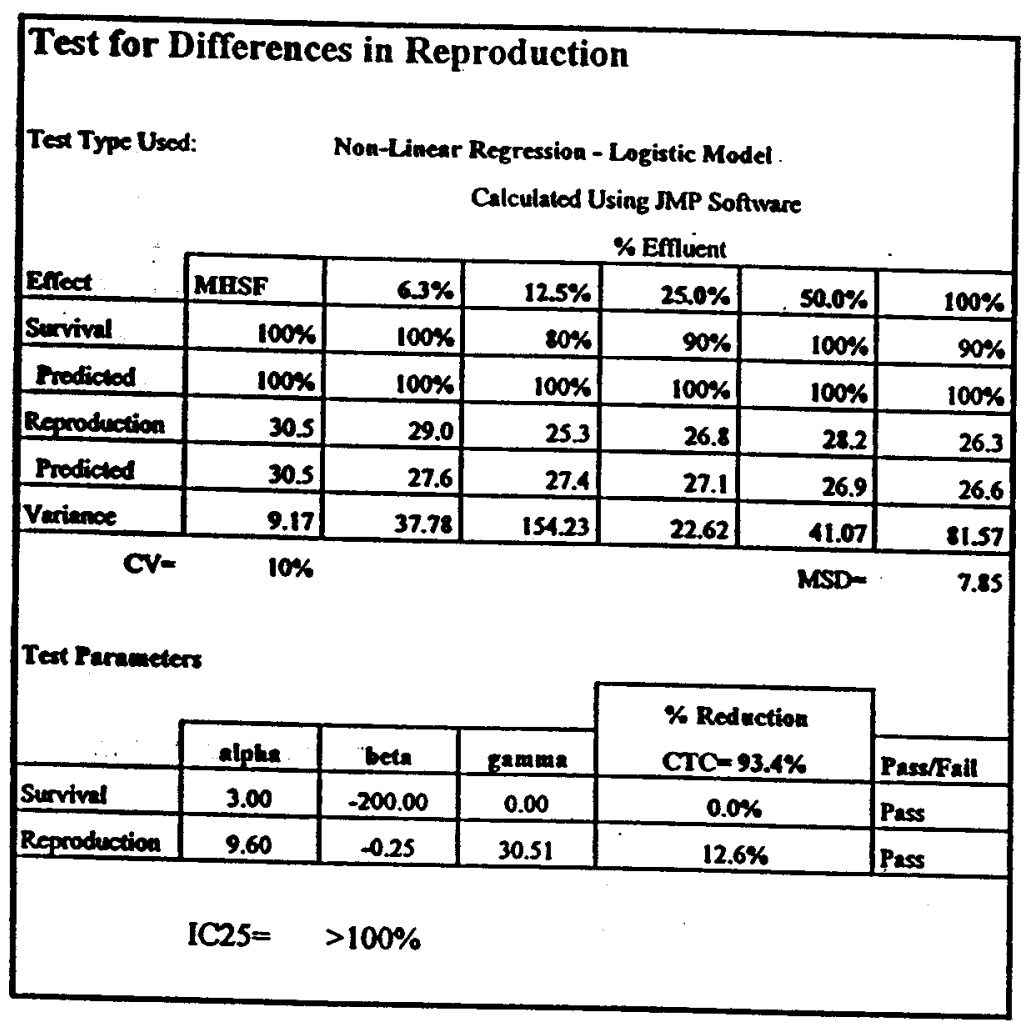

Reproduction vs. Effluent Conc.

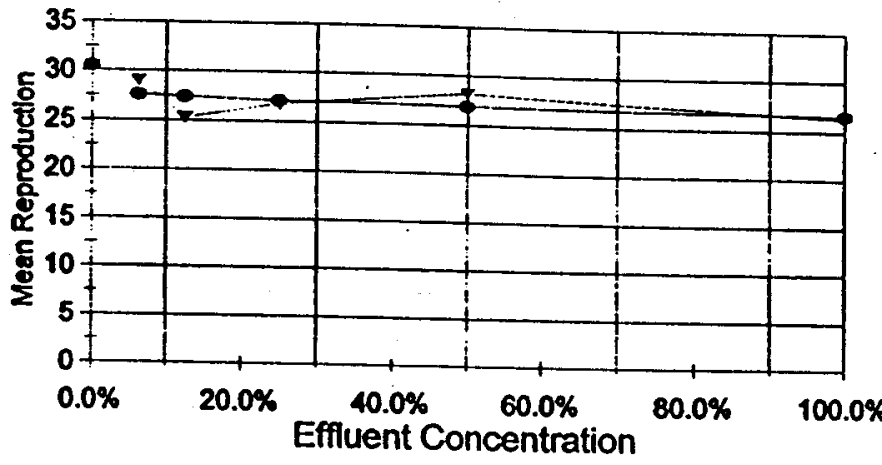

$\rightarrow$ Actual Reproduction $\rightarrow$ Predicted Reproduction

\section{Summary}

The reductions in survival and reproduction at the CTC are $<25 \%$ - Pass 


\section{CHRONIC DEFINITIVE SURVIVAL AND REPRODUCTION TEST \\ Water Chemistry}

Client: WSRC

Sample Identification: A-01

Test Date: $\quad$ February 5, 2002

pH (After Renewal)

\begin{tabular}{|l|r|r|r|r|r|r|}
\hline \multicolumn{7}{|c|}{ Test Concentration } \\
\hline & Control & $6.25 \%$ & $12.5 \%$ & $25 \%$ & $50 \%$ & $100 \%$ \\
\hline Initial & 7.33 & 7.24 & 7.14 & 7.07 & 7.03 & 7.03 \\
\hline Day 1 & 8.27 & 8.01 & 7.89 & 7.69 & 7.66 & 7.63 \\
\hline Day 2 & 7.61 & 7.34 & 7.25 & 7.26 & 7.34 & 7.39 \\
\hline Day 3 & 6.91 & 6.82 & 7.03 & 7.12 & 7.20 & 7.29 \\
\hline Day 4 & 7.63 & 7.40 & 7.33 & 7.30 & 7.31 & 7.34 \\
\hline Day 5 & 7.69 & 7.61 & 7.50 & 7.47 & 7.39 & 7.32 \\
\hline Day 6 & 7.12 & 7.06 & 7.02 & 6.97 & 7.02 & 7.04 \\
\hline
\end{tabular}

Dissolved Oxygen (After Renewal)

\begin{tabular}{|l|r|r|r|r|r|r|}
\hline & \multicolumn{7}{|c|}{ Test Concentration } \\
\hline Lnitial & 8.4 & 8.4 & 8.4 & 8.4 & 8.4 & 8.3 \\
\hline Day 1 & 8.4 & 8.4 & 8.4 & 8.4 & 8.4 & 8.4 \\
\hline Day 2 & 8.4 & 8.4 & 8.4 & 8.4 & 8.4 & 8.3 \\
\hline Day 3 & 8.4 & 8.3 & 8.4 & 8.2 & 8.2 & 8.2 \\
\hline Day 4 & 8.3 & 8.3 & 8.3 & 8.2 & 8.2 & 8.2 \\
\hline Day 5 & 8.4 & 8.3 & 8.3 & 8.3 & 8.3 & 8.3 \\
\hline Day 6 & 8.3 & 8.4 & 8.4 & 8.4 & 8.3 & 8.3 \\
\hline
\end{tabular}

pH (Before Renewal)
\begin{tabular}{|l|r|r|r|r|r|r|}
\hline \multicolumn{7}{|c|}{ Test Concentration } \\
\hline & Control & $6.25 \%$ & $12.5 \%$ & $25 \%$ & $50 \%$ & $100 \%$ \\
\hline Day 1 & 8.22 & 8.09 & 8.02 & 7.93 & 7.87 & 7.82 \\
\hline Day 2 & 7.72 & 7.52 & 7.47 & 7.43 & 7.42 & 7.45 \\
\hline Day 3 & 7.00 & 6.61 & 6.91 & 7.00 & 7.10 & 7.30 \\
\hline Day 4 & 7.79 & 7.50 & 7.48 & 7.39 & 7.38 & 7.41 \\
\hline Day 5 & 7.80 & 7.77 & 7.64 & 7.66 & 7.61 & 7.61 \\
\hline Day 6 & 7.50 & 7.46 & 7.35 & 7.24 & 7.33 & 7.35 \\
\hline Final & 7.93 & 8.08 & 7.83 & 7.61 & 7.53 & 7.48 \\
\hline
\end{tabular}

Dissolved Oxygen (Before Renewal)

\begin{tabular}{|l|r|r|r|r|r|r|}
\hline & \multicolumn{7}{|c|}{ Test Concentration } \\
\hline Day 1 & 8.4 & 8.3 & 8.3 & 8.3 & 8.4 & 8.1 \\
\hline Day 2 & 8.4 & 8.1 & 8.2 & 8.2 & 8.1 & 8.1 \\
\hline Day 3 & 8.5 & 8.4 & 8.2 & 8.2 & 8.1 & 8.0 \\
\hline Day 4 & 8.4 & 8.4 & 8.3 & 8.2 & 8.3 & 8.1 \\
\hline Day 5 & 8.3 & 8.4 & 8.3 & 8.3 & 8.3 & 8.2 \\
\hline Day 6 & 8.3 & 8.3 & 8.3 & 8.3 & 8.3 & 8.2 \\
\hline Final & 8.9 & 8.3 & 8.3 & 8.3 & 8.3 & 8.1 \\
\hline
\end{tabular}

Test Results Reviewed and Approved By:

Temperature
\begin{tabular}{|l|r|}
\hline Incubator ${ }^{\circ} \mathrm{C}$ \\
\hline Initia & 24.8 \\
\hline Day 1 & 25.0 \\
\hline Day 2 & 24.6 \\
\hline Day 3 & 25.0 \\
\hline Day 4 & 25.7 \\
\hline Day 5 & 25.3 \\
\hline Day 6 & 25.7 \\
\hline Final & 25.3 \\
\hline
\end{tabular}




\section{Day Chronic Definitive Survival and Reproduction Bioassay}

Method: EPA/600/4-91/002

Test Organism:

Daphnia ambigua

Facility: WSRC

Sample ID: A-11

Aiken County

ETTH: 19176

Sample ID: February 5, 2002 


\section{CHRONIC DEFINITIVE SURVIVAL AND REPRODUCTION TEST Survival and Reproduction Results}

Client:

Sample Identification:

Test Date:

A-11

February 5, 2002
County:

NPDESH:

ETT \#:
Aiken

SC

19176

\begin{tabular}{|c|c|c|c|c|c|c|}
\hline \multicolumn{7}{|c|}{ Reproduction at } \\
\hline Rep. & Day 3/4 & Day 5 & Day 6 & Day 7 & Day 8 & Total \\
\hline 1 & 6 & 0 & 14 & 13 & 0 & 33 \\
\hline 2 & 4 & 9 & 16 & 0 & 0 & 29 \\
\hline 3 & 5 & 9 & 0 & 14 & 0 & 28 \\
\hline 4 & 4 & 11 & 10 & 0 & 0 & 25 \\
\hline 5 & 6 & 0 & 12 & 14 & 0 & 32 \\
\hline 6 & 6 & 0 & 13 & 14 & 0 & 33 \\
\hline 7 & 5 & 0 & 12 & 13 & 0 & 30 \\
\hline 8 & 6 & 0 & 11 & 15 & 0 & 32 \\
\hline 9 & 6 & 0 & 13 & 16 & 0 & 35 \\
\hline 10 & 4 & 0 & 11 & 13 & 0 & 28 \\
\hline
\end{tabular}

\begin{tabular}{|c|c|c|c|c|c|c|}
\hline Reproduction at & \multicolumn{6}{|c|}{$12.5 \%$} \\
\hline Rep. & Day 3/4 & Day 5 & Day 6 & Day 7 & Day 8 & Total \\
\hline 1 & 5 & 0 & 10 & 19 & 0 & 34 \\
\hline 2 & 0 & 4 & 12 & 0 & 0 & 16 \\
\hline 3 & 6 & 0 & 12 & 14 & 0 & 32 \\
\hline 4 & 6 & 9 & 0 & 12 & 0 & 27 \\
\hline 5 & 6 & 1 & 10 & 0 & 0 & 17 \\
\hline 6 & 4 & 2 & 11 & 16 & 0 & 33 \\
\hline 7 & 6 & 0 & 10 & 15 & 0 & 31 \\
\hline 8 & 6 & 0 & 13 & 0 & 0 & 19 \\
\hline 9 & 6 & 0 & 11 & 0 & 0 & 17 \\
\hline 10 & 5 & 0 & 16 & 0 & 0 & 21 \\
\hline
\end{tabular}

\begin{tabular}{|c|c|c|c|c|c|c|}
\hline \multicolumn{2}{|c|}{ Reproduction at } \\
\hline Rep. & Day 3/4 & Day 5 & Day 6 & Day 7 & Day 8 & Toual \\
\hline 1 & 6 & 0 & 10 & 13 & 0 & 29 \\
\hline 2 & 4 & 10 & 16 & 0 & 0 & 30 \\
\hline 3 & 6 & 12 & 0 & 14 & 0 & 32 \\
\hline 4 & 6 & 12 & 17 & 0 & 0 & 35 \\
\hline 5 & 5 & 0 & 11 & 8 & 0 & 24 \\
\hline 6 & 5 & 0 & 12 & 13 & 0 & 30 \\
\hline 7 & 0 & 5 & 10 & 0 & 0 & 15 \\
\hline 8 & 7 & $\mathrm{~L}$ & $\mathrm{~L}$ & $\mathrm{~L}$ & $\mathrm{~L}$ & $\mathrm{~L}$ \\
\hline 9 & 6 & 0 & 12 & 16 & 0 & 34 \\
\hline 10 & 1 & 3 & 14 & 0 & 0 & 18 \\
\hline
\end{tabular}

\begin{tabular}{|c|c|c|c|c|c|c|}
\hline \multicolumn{1}{|c|}{ Reproduction at } & \multicolumn{3}{|c|}{$25 \%$} & \multicolumn{1}{c|}{ Concentration } \\
\hline Rep. & Day 3/4 & Day 5 & Day 6 & Day 7 & Day 8 & Total \\
\hline 1 & 7 & 0 & 12 & 17 & 0 & 36 \\
\hline 2 & 5 & 11 & 15 & 0 & 0 & 31 \\
\hline 3 & 5 & 0 & 12 & 18 & 0 & 35 \\
\hline 4 & 4 & 12 & 16 & 0 & 0 & 32 \\
\hline 5 & 6 & $X$ & $X$ & $X$ & $X$ & 6 \\
\hline 6 & 4 & 0 & 11 & 15 & 0 & 30 \\
\hline 7 & 6 & 0 & 10 & 9 & 0 & 25 \\
\hline 8 & 6 & 0 & 10 & 0 & 0 & 16 \\
\hline 9 & 4 & 0 & 10 & 14 & 0 & 28 \\
\hline 10 & 0 & 6 & 13 & 0 & 0 & 19 \\
\hline
\end{tabular}

\begin{tabular}{|c|c|c|c|c|c|c|}
\hline \multicolumn{1}{|c|}{ Reproduction at } & \multicolumn{3}{c|}{$50 \%$} & Concentration \\
\hline Rep. & Day 3/4 & Day 5 & Day 6 & Day 7 & Day & Tolal \\
\hline 1 & 7 & 0 & 12 & 15 & 0 & 34 \\
\hline 2 & 0 & $X$ & $X$ & $X$ & $X$ & 0 \\
\hline 3 & 5 & 0 & 11 & 14 & 0 & 30 \\
\hline 4 & 5 & 0 & 15 & 12 & 0 & 32 \\
\hline 5 & 5 & 0 & 12 & 14 & 0 & 31 \\
\hline 6 & 6 & 0 & 11 & 0 & 0 & 17 \\
\hline 7 & 4 & $L$ & $L$ & $L$ & $L$ & $L$ \\
\hline 8 & 6 & 0 & 9 & $X$ & $X$ & 15 \\
\hline 9 & 0 & 5 & 11 & 0 & 0 & 16 \\
\hline 10 & 0 & 5 & 11 & 0 & 0 & 16 \\
\hline
\end{tabular}

\begin{tabular}{|c|c|c|c|c|c|c}
\hline \multicolumn{1}{|c|}{ Reproduction at } & \multicolumn{1}{c}{ Concentration } \\
\hline Rep. & Day 3/4 & Day 5 & Day 6 & Day 7 & Day 8 & Total \\
\hline 1 & 5 & 0 & 12 & 14 & 0 & 31 \\
\hline 2 & 5 & 7 & 12 & 0 & 0 & 24 \\
\hline 3 & 6 & 0 & 12 & 10 & 0 & 28 \\
\hline 4 & 3 & 10 & 15 & 0 & 0 & 28 \\
\hline 5 & 7 & 0 & 11 & 10 & 0 & 28 \\
\hline 6 & 6 & 11 & 16 & 0 & 0 & 33 \\
\hline 7 & 5 & L & L & L & L & L \\
\hline 8 & 6 & 0 & 14 & 0 & 0 & 20 \\
\hline 9 & 7 & 0 & 12 & 13 & 0 & 32 \\
\hline 10 & 6 & 0 & 15 & 0 & 0 & 21 \\
\hline
\end{tabular}




\section{CHRONIC DEFINITIVE SURVIVAL AND REPRODUCTION TEST \\ Statistical Analyses}

Client: WSRC

Sample Identification:

Test Date:

February 5, 2002

\section{Test for Normality}

Test Used: Kolmogorov D

$\begin{array}{ll}D= & \text { NA } \\ \text { critical } & \text { NA }\end{array}$

NA
Test for Homogeneity of Variance

Test Used: Bartlett's Test

$B=N A$

critical NA

NA
Reproduction vs. Effluent Conc.

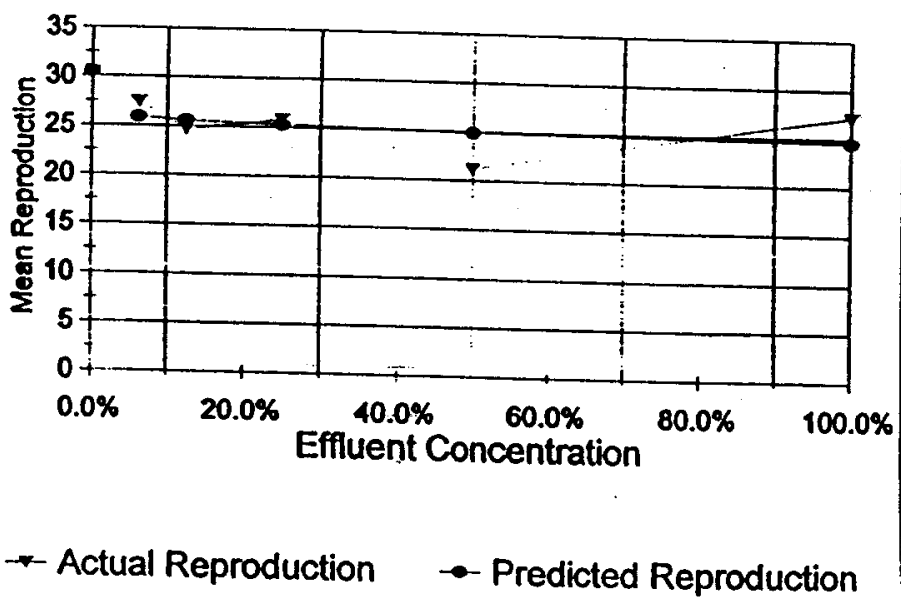

Test Parnecters

\begin{tabular}{|c|c|c|c|c|c|}
\hline & alpha & beta & Eamana & $\begin{array}{l}\text { X Reduction } \\
\text { CTC }=98.1 \%\end{array}$ & PaserFall \\
\hline Survival & 3.00 & -200.00 & 0.00 & $0.0 \%$ & Puss \\
\hline Reproduction & 7.75 & -0.25 & 30.52 & $19.4 \%$ & Pass \\
\hline
\end{tabular}

Summary

The reductions in survival and reproduction at the CTC are $<25 \%$ - Pass 


\section{CHRONIC DEFINITIVE SURVIVAL AND REPRODUCTION TEST \\ Water Chemistry}

Client: WSRC

Sample Identification:

Test Date:

A-11

February 5, 2002

pH (After Renewal)

\begin{tabular}{|l|r|r|r|r|r|r|}
\hline & Control & $6.25 \%$ & $12.5 \%$ & $25 \%$ & $50 \%$ & $100 \%$ \\
\hline Initial & 7.33 & 7.08 & 7.07 & 7.11 & 7.15 & 7.15 \\
\hline Day 1 & 8.27 & 8.13 & 7.02 & 7.27 & 7.15 & 6.98 \\
\hline Day 2 & 7.61 & 7.29 & 7.22 & 7.16 & 7.13 & 7.15 \\
\hline Day 3 & 6.91 & 7.22 & 8.48 & 7.61 & 7.30 & 7.57 \\
\hline Day 4 & 7.63 & 7.32 & 7.30 & 7.24 & 7.20 & 7.14 \\
\hline Day 5 & 7.69 & 8.83 & 8.85 & 8.86 & 8.66 & 8.50 \\
\hline Day 6 & 7.12 & 7.38 & 7.32 & 7.21 & 7.21 & 7.17 \\
\hline
\end{tabular}

Dissolved Oxygen (After Renewal)

\begin{tabular}{|l|r|r|r|r|r|r|}
\hline & \multicolumn{7}{|c|}{ Test Concentration } \\
\hline Initial & 8.4 & 8.4 & 8.3 & 8.3 & 8.3 & 8.3 \\
\hline Day 1 & 8.4 & 8.3 & 8.3 & 8.3 & 8.3 & 8.3 \\
\hline Day 2 & 8.4 & 8.4 & 8.3 & 8.3 & 8.3 & 8.1 \\
\hline Day 3 & 8.4 & 8.6 & 8.6 & 8.6 & 8.7 & 8.7 \\
\hline Day 4 & 8.3 & 8.5 & 8.5 & 8.5 & 8.5 & 8.5 \\
\hline Day 5 & 8.4 & 8.4 & 8.3 & 8.3 & 8.3 & 8.3 \\
\hline Day 6 & 8.3 & 8.4 & 8.3 & 8.3 & 8.3 & 8.3 \\
\hline
\end{tabular}

pH (Before Renewal)
\begin{tabular}{|l|r|r|r|r|r|r|}
\hline \multicolumn{7}{|c|}{ Test Concentration } \\
\hline & Control & $6.25 \%$ & $12.5 \%$ & $25 \%$ & $50 \%$ & $100 \%$ \\
\hline Day 1 & 8.22 & 7.56 & 7.57 & 7.46 & 7.39 & 7.40 \\
\hline Day 2 & 7.72 & 7.40 & 7.34 & 7.31 & 7.31 & 7.30 \\
\hline Day 3 & 7.00 & 7.38 & 7.18 & 7.18 & 7.08 & 7.28 \\
\hline Day 4 & 7.79 & 7.43 & 7.38 & 7.34 & 7.32 & 7.29 \\
\hline Day 5 & 7.80 & 8.89 & 9.00 & 9.00 & 8.81 & 8.68 \\
\hline Day 6 & 7.50 & 7.73 & 7.67 & 7.65 & 7.59 & 7.57 \\
\hline Final & 7.93 & 7.18 & 6.20 & 7.44 & 7.61 & 7.17 \\
\hline
\end{tabular}

Dissolved Oxygen (Before Renewal)
\begin{tabular}{|l|r|r|r|r|r|r|}
\hline \multicolumn{7}{|c|}{ Test Concentration } \\
\hline & Control & $6.25 \%$ & $12.5 \%$ & $25 \%$ & $50 \%$ & $100 \%$ \\
\hline Day 1 & 8.4 & 8.4 & 8.4 & 8.3 & 8.3 & 8.3 \\
\hline Day 2 & 8.4 & 8.3 & 8.2 & 8.2 & 8.2 & 8.2 \\
\hline Day 3 & 8.5 & 8.5 & 8.6 & 8.7 & 8.7 & 8.7 \\
\hline Day 4 & 8.4 & 8.5 & 8.5 & 8.5 & 8.5 & 8.6 \\
\hline Day 5 & 8.3 & 8.4 & 8.4 & 8.4 & 8.4 & 8.4 \\
\hline Day 6 & 8.3 & 8.3 & 8.6 & 8.3 & 8.3 & 8.2 \\
\hline Final & 8.9 & 8.4 & 8.3 & 8.3 & 8.2 & 8.2 \\
\hline
\end{tabular}

Test Results Reviewed and Approved By:

Temperature
\begin{tabular}{|l|r|}
\hline \multicolumn{2}{|c|}{ Incubator ${ }^{\circ} \mathrm{C}$} \\
\hline Initial & 24.8 \\
\hline Day 1 & 25.0 \\
\hline Day 2 & 24.6 \\
\hline Day 3 & 25.0 \\
\hline Day 4 & 25.7 \\
\hline Day 5 & 25.3 \\
\hline Day 6 & 25.7 \\
\hline Final & 25.3 \\
\hline
\end{tabular}




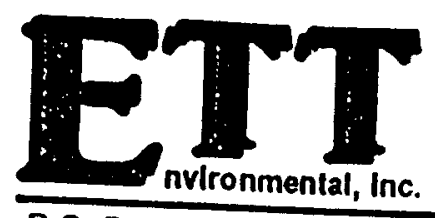

P.O. Box 16414, Greenville, SC 29606

(864) 877-6942 - FAX (864) 877-6938

4 Craftsman Court, Greer, SC 29650

\section{Day Chronic Definitive Survival and Reproduction Bioassay \\ Method: EPA/600/4-91/002}

Test Organism:

Daphnia ambigua

Facility: WSRC

Sample ID: G-10

Aiken County

ETTH: 19177

Sample ID: February 5, 2002 


\section{CHRONIC DEFINITIVE SURVIVAL AND REPRODUCTION TEST Survival and Reproduction Results}

Client:

WSRC

Sample Identification:

Test Date:

G-10

February 5, 2002

\begin{tabular}{|c|c|c|c|c|c|c|}
\hline \multicolumn{7}{|c|}{ Reproduction at } \\
\hline Rep. & Day 3/4 & Day 5 & Day 6 & Day 7 & Day 8 & Total \\
\hline 1 & 3 & 11 & 15 & 0 & 0 & 29 \\
\hline 2 & 5 & 1 & 14 & 13 & 0 & 33 \\
\hline 3 & 3 & 11 & 16 & 0 & 0 & 30 \\
\hline 4 & $X$ & $X$ & $X$ & $X$ & $X$ & 0 \\
\hline 5 & 6 & 10 & 0 & 14 & 0 & 30 \\
\hline 6 & 5 & 1 & 15 & 12 & 0 & 33 \\
\hline 7 & 11 & 0 & 12 & 0 & 0 & 23 \\
\hline 8 & 3 & 0 & 11 & 15 & 0 & 29 \\
\hline 9 & 3 & 8 & 12 & 0 & 0 & 23 \\
\hline 10 & $X$ & $X$ & $X$ & $X$ & $X$ & 0 \\
\hline
\end{tabular}

\begin{tabular}{|c|c|c|c|c|c|c|}
\hline Reproduction at & \multicolumn{1}{|c|}{$12.5 \%$} & \multicolumn{3}{c|}{ Concentration } \\
\hline Rep. & Day 3/4 & Day 5 & Day 6 & Day 7 & Day 8 & Total \\
\hline 1 & 5 & 1 & 11 & 15 & 0 & 32 \\
\hline 2 & 5 & 0 & 10 & 5 & 0 & 20 \\
\hline 3 & 5 & 1 & 11 & 0 & 0 & 17 \\
\hline 4 & 5 & 0 & 11 & 17 & 0 & 33 \\
\hline 5 & 6 & 0 & 12 & 16 & 0 & 34 \\
\hline 6 & 4 & 0 & 11 & 12 & 0 & 27 \\
\hline 7 & 0 & 5 & 12 & 0 & 0 & 17 \\
\hline 8 & 5 & 0 & 10 & 14 & 0 & 29 \\
\hline 9 & 0 & 4 & 11 & 0 & 0 & 15 \\
\hline 10 & 0 & 5 & 8 & 0 & 0 & 13 \\
\hline
\end{tabular}

\begin{tabular}{|c|c|c|c|c|c|c|}
\hline Reproduction at & \multicolumn{6}{|c|}{$50 \%$} \\
\hline Rep. & Day 3/4 & Day 5 & Day 6 & Day 7 & Duy & Toual \\
\hline 1 & 5 & 0 & 14 & $X$ & $X$ & 19 \\
\hline 2 & 5 & 0 & 16 & 14 & 0 & 35 \\
\hline 3 & 5 & 0 & 15 & 16 & 0 & 36 \\
\hline 4 & 4 & 0 & 16 & 15 & 0 & 35 \\
\hline 5 & 5 & 0 & 10 & 8 & 0 & 23 \\
\hline 6 & 0 & 5 & 11 & 0 & 0 & 16 \\
\hline 7 & 4 & 0 & 9 & 19 & 0 & 32 \\
\hline 8 & 4 & 0 & 14 & 15 & 0 & 33 \\
\hline 9 & 0 & 6 & 12 & 0 & 0 & 18 \\
\hline 10 & 0 & 5 & 15 & 0 & 0 & 20 \\
\hline
\end{tabular}

County:

NPDESH :

ETT \#:

Aiken

SC

19177

\begin{tabular}{|c|c|c|c|c|c|c|}
\hline \multicolumn{2}{|c|}{ Reproduction at } \\
\hline Rep. & Day 3/4 & Day 5 & Day 6 & Day 7 & Day 8 & Tocal \\
\hline 1 & 4 & 1 & 10 & 14 & 0 & 29 \\
\hline 2 & 5 & 0 & 10 & $X$ & $X$ & 15 \\
\hline 3 & 4 & 1 & 12 & 15 & 0 & 32 \\
\hline 4 & 5 & 1 & 12 & 0 & 0 & 18 \\
\hline 5 & 6 & 0 & 12 & 15 & 0 & 33 \\
\hline 6 & 3 & 1 & 11 & 5 & $X$ & 20 \\
\hline 7 & 0 & 4 & 11 & 0 & 0 & 15 \\
\hline 8 & 5 & 0 & 12 & 14 & 0 & 31 \\
\hline 9 & 0 & 3 & 11 & $X$ & $X$ & 14 \\
\hline 10 & 0 & 5 & 12 & 3 & 0 & 20 \\
\hline
\end{tabular}

\begin{tabular}{|c|c|c|c|c|c|c|}
\hline \multicolumn{1}{|c|}{ Reproduction at } \\
\hline Rep. & Day 3/4 & Day 5 & Day 6 & Day 7 & Day 8 & Total \\
\hline 1 & 5 & 0 & 12 & 14 & 0 & 31 \\
\hline 2 & 5 & 4 & 13 & 10 & 0 & 32 \\
\hline 3 & 6 & 0 & 9 & $X$ & $X$ & 15 \\
\hline 4 & 5 & 1 & 12 & 13 & 0 & 31 \\
\hline 5 & 6 & 0 & 13 & 15 & 0 & 34 \\
\hline 6 & 4 & 0 & 12 & 0 & 0 & 16 \\
\hline 7 & 3 & 0 & 13 & 0 & 0 & 16 \\
\hline 8 & 3 & 0 & 14 & 13 & 0 & 30 \\
\hline 9 & 0 & 6 & $X$ & $X$ & $X$ & 6 \\
\hline 10 & 0 & 4 & 13 & 12 & 0 & 29 \\
\hline
\end{tabular}

\begin{tabular}{|c|c|c|c|c|c|c|}
\hline \multicolumn{2}{|c|}{ Reproduction at } & \multicolumn{2}{c|}{$100 \%$} \\
\hline Rep. & Day 3/4 & Day 5 & Day 6 & Day 7 & Day 8 & Total \\
\hline 1 & 5 & 0 & 11 & 15 & 0 & 31 \\
\hline 2 & 4 & 0 & 10 & 14 & 0 & 28 \\
\hline 3 & 3 & 0 & 13 & 0 & 0 & 16 \\
\hline 4 & 3 & 0 & 14 & 0 & 0 & 17 \\
\hline 5 & 4 & 0 & 14 & 14 & 0 & 32 \\
\hline 6 & 5 & 0 & 12 & 15 & 0 & 32 \\
\hline 7 & 2 & 0 & 15 & 8 & 0 & 25 \\
\hline 8 & 5 & 0 & 17 & $X$ & $X$ & 22 \\
\hline 9 & 0 & 6 & 15 & 0 & 0 & 21 \\
\hline 10 & 4 & 0 & 13 & 14 & 0 & 31 \\
\hline
\end{tabular}




\section{CHRONIC DEFINITIVE SURVIVAL AND REPRODUCTION TEST \\ Statistical Analyses}

Client:

Sample Identification:

Test Date:
WSRC

G-10

February 5, 2002

\section{Test for Normality}

Test Used: Kolmogorov D

$\begin{array}{ll}D= & N A \\ \text { critical } & \text { NA }\end{array}$

NA
Test for Homogeneity of Variance

Test Used: Bartlett's Test

$B=N A$

critical NA

NA

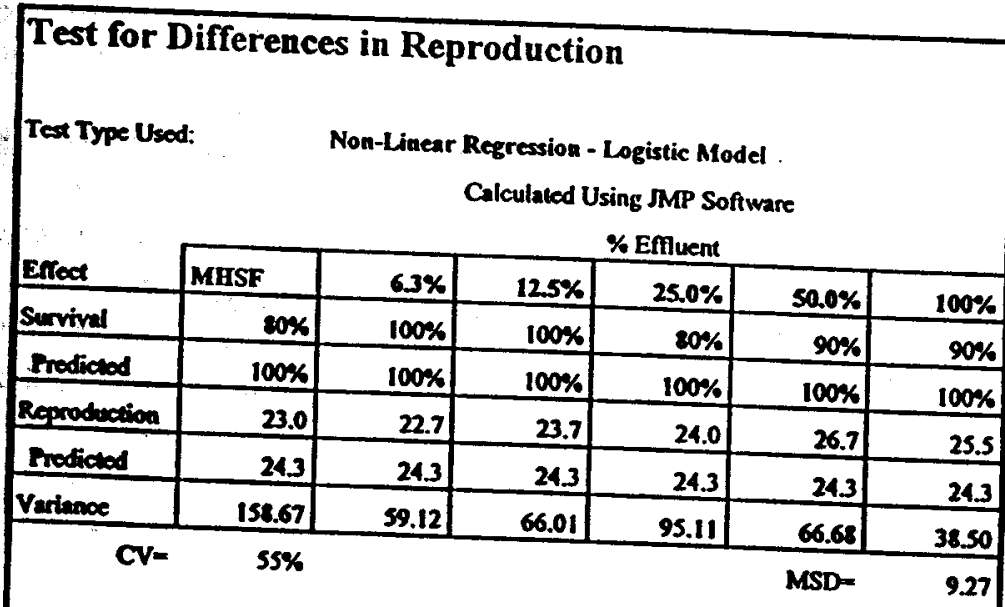

Test Parameters

\begin{tabular}{|c|c|c|c|c|c|}
\hline$\therefore \quad \therefore$ & alpha & beta & gamma & $\begin{array}{l}\% \text { Reduction } \\
\text { CTC }=100 \%\end{array}$ & Pass/Fail \\
\hline Survival & 3.00 & -200.00 & 0.00 & $0.0 \%$ & Pass \\
\hline Reproduction & 4.78 & -200.00 & 24.27 & $0.0 \%$ & Pass \\
\hline & IC25 $=$ & $>100 \%$ & & & \\
\hline
\end{tabular}

\section{Reproduction vs. Effluent Conc.}

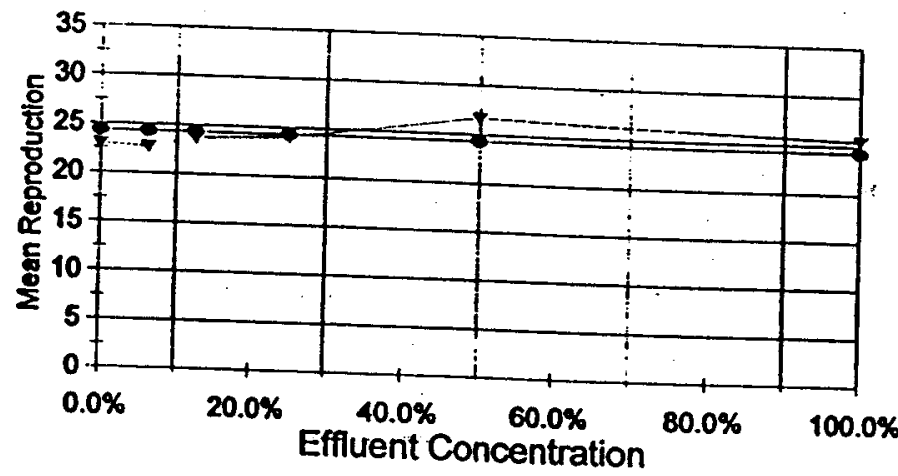

$\rightarrow$ Actual Reproduction $\rightarrow$ Predicted Reproduction 


\section{CHRONIC DEFINITIVE SURVIVAL AND REPRODUCTION TEST \\ Water Chemistry}

Client:

WSRC

Sample Identification:

G-10

Test Date:

February 5, 2002

PH (After Renewal)
\begin{tabular}{|l|r|r|r|r|r|r|}
\hline \multicolumn{7}{|c|}{ Test Concentration } \\
\hline & Control & $6.25 \%$ & $12.5 \%$ & $25 \%$ & $50 \%$ & $100 \%$ \\
\hline Initial & 7.33 & 7.92 & 7.79 & 7.68 & 7.63 & 7.65 \\
\hline Day 1 & 8.27 & 7.52 & 7.44 & 7.50 & 7.56 & 7.73 \\
\hline Day 2 & 7.61 & 7.75 & 7.64 & 7.52 & 7.46 & 7.64 \\
\hline Day 3 & 6.91 & 8.34 & 8.34 & 7.95 & 7.91 & 7.94 \\
\hline Day 4 & 7.63 & 7.93 & 7.85 & 7.64 & 7.60 & 7.61 \\
\hline Day 5 & 7.69 & 8.09 & 7.88 & 7.77 & 7.76 & 7.62 \\
\hline Day 6 & 7.12 & 7.44 & 7.38 & 7.48 & 7.73 & 7.81 \\
\hline
\end{tabular}

pH (Before Renewal)
\begin{tabular}{|l|r|r|r|r|r|r|}
\hline & Control & $6.25 \%$ & $12.5 \%$ & $25 \%$ & $50 \%$ & $100 \%$ \\
\hline Day 1 & 8.22 & 8.01 & 7.85 & 7.79 & 7.70 & 7.76 \\
\hline Day 2 & 7.72 & 7.91 & 7.79 & 7.70 & 7.62 & 7.61 \\
\hline Day 3 & 7.00 & 8.93 & 8.77 & 8.25 & 8.07 & 7.99 \\
\hline Day 4 & 7.79 & 8.00 & 7.91 & 7.63 & 7.61 & 7.61 \\
\hline Day 5 & 7.80 & 8.32 & 8.17 & 8.04 & 7.93 & 7.90 \\
\hline Day 6 & 7.50 & 7.59 & 7.45 & 7.51 & 7.79 & 7.92 \\
\hline Final & 7.93 & 7.43 & 7.35 & 7.43 & 7.60 & 7.93 \\
\hline
\end{tabular}

Dissolved Oxygen (After Renewal)

\begin{tabular}{|l|r|r|r|r|r|r|}
\hline & \multicolumn{7}{|c|}{ Test Concentration } & $6.25 \%$ & $12.5 \%$ & $25 \%$ & $50 \%$ & $100 \%$ \\
\hline Initial & 8.4 & 8.4 & 8.4 & 8.3 & 8.4 & 8.3 \\
\hline Day 1 & 8.4 & 8.4 & 8.4 & 8.4 & 8.4 & 8.4 \\
\hline Day 2 & 8.4 & 8.4 & 8.4 & 8.4 & 8.4 & 8.3 \\
\hline Day 3 & 8.4 & 8.5 & 8.4 & 8.4 & 8.4 & 8.4 \\
\hline Day 4 & 8.3 & 8.5 & 8.4 & 8.4 & 8.4 & 8.4 \\
\hline Day 5 & 8.4 & 8.4 & 8.4 & 8.4 & 8.4 & 8.3 \\
\hline Day 6 & 8.3 & 8.4 & 8.4 & 8.4 & 8.4 & 8.4 \\
\hline
\end{tabular}

Dissolved Oxygen (Before Renewal)
\begin{tabular}{|l|r|r|r|r|r|r|}
\hline \multicolumn{7}{|c|}{ Test Concentration } \\
\hline & Control & $6.25 \%$ & $12.5 \%$ & $25 \%$ & $50 \%$ & $100 \%$ \\
\hline Day 1 & 8.4 & 8.4 & 8.3 & 8.3 & 8.3 & 8.1 \\
\hline Day 2 & 8.4 & 8.3 & 8.3 & 8.4 & 8.3 & 8.2 \\
\hline Day 3 & 8.5 & 8.4 & 8.3 & 8.3 & 8.2 & 8.2 \\
\hline Day 4 & 8.4 & 8.3 & 8.2 & 8.2 & 8.2 & 8.1 \\
\hline Day 5 & 8.3 & 8.3 & 8.2 & 8.7 & 8.1 & 8.0 \\
\hline Day 6 & 8.3 & 8.3 & 8.3 & 8.2 & 8.2 & 8.1 \\
\hline Final & 8.9 & 8.4 & 8.3 & 8.2 & 8.2 & 8.2 \\
\hline
\end{tabular}

Test Results Reviewed and Approved By:

Temperature
\begin{tabular}{|l|r|}
\hline \multicolumn{2}{|c|}{ Incubator ${ }^{\circ} \mathrm{C}$} \\
\hline Initia & 24.8 \\
\hline Day 1 & 25.0 \\
\hline Day 2 & 24.6 \\
\hline Day 3 & 25.0 \\
\hline Day 4 & 25.7 \\
\hline Day 5 & 25.3 \\
\hline Day 6 & 25.7 \\
\hline Final & 25.3 \\
\hline
\end{tabular}




\section{Day Chronic Definitive Survival and Reproduction Bioassay Method: EPA/600/4-91/002}

Test Organism:

\author{
Daphnia ambigua
}

Facility: WSRC

Sample ID: A-01

Aiken County

ETT\#: T19343

Sample ID: February 26, 2002 


\section{CHRONIC DEFINITIVE SURVIVAL AND REPRODUCTION TEST \\ Survival and Reproduction Results}

Client:

Sample Identification:

Test Date: $\quad$ February 26, 2002

WSRC

\begin{tabular}{|c|c|c|c|c|c|c|}
\hline Reproduction at & \multicolumn{7}{|c|}{ Control } \\
\hline Rep. & Day 3/4 & Day 5 & Day 6 & Day 7 & Day 8 & Total \\
\hline 1 & 0 & 5 & 14 & $X$ & $X$ & 19 \\
\hline 2 & 7 & 0 & 10 & 11 & 0 & 28 \\
\hline 3 & 4 & 0 & 9 & 13 & 0 & 26 \\
\hline 4 & 0 & 5 & 10 & 0 & 14 & 29 \\
\hline 5 & 5 & 0 & 12 & $X$ & $X$ & 17 \\
\hline 6 & 5 & 0 & 14 & 15 & 0 & 34 \\
\hline 7 & 4 & 0 & 8 & 11 & 0 & 23 \\
\hline 8 & 6 & 0 & 13 & 12 & 0 & 31 \\
\hline 9 & 5 & 0 & 14 & 12 & 0 & 31 \\
\hline 10 & 4 & 0 & 10 & 11 & 0 & 25 \\
\hline
\end{tabular}

\begin{tabular}{|c|c|c|c|c|c|c|}
\hline \multicolumn{1}{|c|}{ Reproduction at } \\
\hline Rep. & Day 3/4 & Day 5 & Day 6 & Day 7 & Day 8 & Toual \\
\hline 1 & 0 & 6 & 7 & 0 & 8 & 21 \\
\hline 2 & $\mathrm{~L}$ & $\mathrm{~L}$ & $\mathrm{~L}$ & $\mathrm{~L}$ & $\mathrm{~L}$ & $\mathrm{~L}$ \\
\hline 3 & 5 & 0 & 10 & 12 & 0 & 27 \\
\hline 4 & 6 & 0 & 13 & $\mathrm{X}$ & $\mathrm{X}$ & 19 \\
\hline 5 & 6 & 0 & 11 & 13 & 0 & 30 \\
\hline 6 & $\mathrm{~L}$ & $\mathrm{~L}$ & $\mathrm{~L}$ & $\mathrm{~L}$ & $\mathrm{~L}$ & $\mathrm{~L}$ \\
\hline 7 & 5 & 0 & 10 & 13 & 0 & 28 \\
\hline 8 & $\mathrm{~L}$ & $\mathrm{~L}$ & $\mathrm{~L}$ & $\mathrm{~L}$ & $\mathrm{~L}$ & $\mathrm{~L}$ \\
\hline 9 & 5 & 0 & 11 & 12 & 0 & 28 \\
\hline 10 & 5 & 0 & 8 & 5 & 0 & 18 \\
\hline
\end{tabular}

\begin{tabular}{|c|c|c|c|c|c|c|}
\hline Reproduction at & \multicolumn{6}{|c|}{ Co\% } \\
\hline Rep. & Day 3/4 & Day 5 & Day 6 & Day 7 & Day & Tocal \\
\hline 1 & 5 & 0 & 9 & 13 & 0 & 27 \\
\hline 2 & 5 & 0 & 11 & 15 & 0 & 31 \\
\hline 3 & $\mathrm{~L}$ & $\mathrm{~L}$ & $\mathrm{~L}$ & $\mathrm{~L}$ & $\mathrm{~L}$ & $\mathrm{~L}$ \\
\hline 4 & 4 & 0 & 10 & 14 & 0 & 28 \\
\hline 5 & 6 & 0 & 11 & 0 & 11 & 28 \\
\hline 6 & 6 & 0 & 12 & 5 & 0 & 23 \\
\hline 7 & 5 & 0 & 14 & 11 & 0 & 30 \\
\hline 8 & 6 & 0 & 13 & 14 & 0 & 33 \\
\hline 9 & 5 & 0 & 12 & $X$ & $\mathrm{X}$ & 17 \\
\hline 10 & 5 & 0 & 10 & 13 & 0 & 28 \\
\hline
\end{tabular}

$\begin{array}{ll}\text { County: } & \text { Aiken } \\ \text { NPDES\#: } & \text { SC }\end{array}$

ETT \#:

T19343

\begin{tabular}{|c|c|c|c|c|c|c|}
\hline \multicolumn{2}{|c|}{ Reproduction at } & \multicolumn{6}{|c|}{$6.25 \%$} & \multicolumn{2}{c|}{ Concentration } \\
\hline Rep. & Day 3/4 & Day 5 & Day 6 & Day 7 & Day 8 & Total \\
\hline 1 & $\mathrm{~L}$ & $\mathrm{~L}$ & $\mathrm{~L}$ & $\mathrm{~L}$ & $\mathrm{~L}$ & $\mathrm{~L}$ \\
\hline 2 & $\mathrm{~L}$ & $\mathrm{~L}$ & $\mathrm{~L}$ & $\mathrm{~L}$ & $\mathrm{~L}$ & $\mathrm{~L}$ \\
\hline 3 & $\mathrm{~L}$ & $\mathrm{~L}$ & $\mathrm{~L}$ & $\mathrm{~L}$ & $\mathrm{~L}$ & $\mathrm{~L}$ \\
\hline 4 & 5 & 0 & 12 & 12 & 0 & 29 \\
\hline 5 & $\mathrm{~L}$ & $\mathrm{~L}$ & $\mathrm{~L}$ & $\mathrm{~L}$ & $\mathrm{~L}$ & $\mathrm{~L}$ \\
\hline 6 & 6 & 0 & 8 & 0 & 12 & 26 \\
\hline 7 & 5 & 0 & 10 & 13 & 0 & 28 \\
\hline 8 & 5 & 0 & 9 & 12 & 0 & 26 \\
\hline 9 & 6 & 0 & 10 & 11 & 0 & 27 \\
\hline 10 & 6 & 0 & 12 & $\mathrm{X}$ & $\mathrm{X}$ & 18 \\
\hline
\end{tabular}

\begin{tabular}{|c|c|c|c|c|c|c|}
\hline \multicolumn{2}{|c|}{ Reproduction at } \\
\hline Rep. & Day 3/4 & Day 5 & Day 6 & Day 7 & Day 8 & Total \\
\hline 1 & 0 & 0 & 7 & 0 & 13 & 20 \\
\hline 2 & 5 & 0 & 9 & $\mathrm{X}$ & $\mathrm{X}$ & $\mathrm{L}$ \\
\hline 3 & $\mathrm{~L}$ & $\mathrm{~L}$ & $\mathrm{~L}$ & $\mathrm{~L}$ & $\mathrm{~L}$ & 0 \\
\hline 4 & 6 & 0 & 10 & 12 & 0 & 28 \\
\hline 5 & $\mathrm{~L}$ & $\mathrm{~L}$ & $\mathrm{~L}$ & $\mathrm{~L}$ & $\mathrm{~L}$ & 0 \\
\hline 6 & 5 & 0 & 13 & 17 & 0 & $\mathrm{~L}$ \\
\hline 7 & 6 & 0 & 14 & 14 & 0 & 34 \\
\hline 8 & 5 & 0 & 12 & 10 & 0 & $\mathrm{~L}$ \\
\hline 9 & $\mathrm{~L}$ & $\mathrm{~L}$ & $\mathrm{~L}$ & $\mathrm{~L}$ & $\mathrm{~L}$ & 0 \\
\hline 10 & $\mathrm{~L}$ & $\mathrm{~L}$ & $\mathrm{~L}$ & $\mathrm{~L}$ & $\mathrm{~L}$ & 0 \\
\hline
\end{tabular}

\begin{tabular}{|c|c|c|c|c|c|c|}
\hline \multicolumn{2}{|c|}{ Reproduction at } & \multicolumn{3}{c}{ Concentration } \\
\hline Rep. & Day 3/4 & Day 5 & Day 6 & Day 7 & Day 8 & Total \\
\hline 1 & 0 & 0 & 0 & 0 & 0 & 0 \\
\hline 2 & 0 & 5 & 10 & 0 & 8 & 23 \\
\hline 3 & 1 & 0 & 11 & 0 & 15 & $\mathrm{~L}$ \\
\hline 4 & 5 & 0 & 13 & 14 & 0 & 32 \\
\hline 5 & 4 & 1 & 11 & 14 & 0 & 30 \\
\hline 6 & 6 & 0 & 13 & 0 & 14 & 33 \\
\hline 7 & 6 & 0 & 13 & 14 & 0 & 33 \\
\hline 8 & 5 & 0 & 12 & 14 & 0 & 31 \\
\hline 9 & 6 & 0 & 12 & 15 & 0 & 33 \\
\hline 10 & 6 & 0 & 10 & 10 & 0 & 26 \\
\hline
\end{tabular}




\section{CHRONIC DEFINITIVE SURVIVAL AND REPRODUCTION TEST \\ Statistical Analyses}

Client: WSRC

Sample Identification: A-01

Test Date: $\quad$ Fel, $\quad$ ruary 26, 2002

\section{Test for Normality}

Test Used: Shapiro-Wilks

$$
\begin{array}{ll}
W= & \text { NA } \\
\text { critical } & \text { NA }
\end{array}
$$

\begin{tabular}{|c|c|c|c|c|c|c|}
\hline \multicolumn{2}{|c|}{ Teat Type Uned: } & \multicolumn{5}{|c|}{$\begin{array}{l}\text { Non-Linear Regression - Logistic Model } \\
\text { Celculated Using JMP Software } \\
\text { \%EAlwent }\end{array}$} \\
\hline Enfeat: & MFSF & $6.3 \%$ & $12.5 \%$ & $250 \%$ & $50.0 \%$ & $100 \%$ \\
\hline survivel & $20 \%$ & $100 \%$ & $89 \%$ & $86 \%$ & $59 \%$ & $100 \%$ \\
\hline Prodicted & $100 x$ & $100 \%$ & $100 \%$ & $100 \%$ & $100 x$ & $100 \%$ \\
\hline Reproduction & 26.3 & 25.7 & 24.8 & 26.7 & 27.2 & 26.8 \\
\hline Prodictod & 26.0 & 26.0 & 26.0 & 26.0 & 26.0 & 26.0 \\
\hline Verience & 29.57 & 15.47 & 18.94 & 55.90 & 22.4 & 100.40 \\
\hline$C V=$ & $21 \%$ & & & & $\mathbf{M S D}=$ & 6.72 \\
\hline
\end{tabular}

NA

\begin{tabular}{|c|c|c|c|c|c|}
\hline & alpha & beta & gamma & $\begin{array}{l}\% \text { Reduction } \\
\text { CTC }=93.4 \%\end{array}$ & Pasarpall \\
\hline Survival & 3.00 & -200.00 & 0.00 & $0.0 \%$ & Pess \\
\hline Reproduction & 4.78 & -48000.00 & 26.00 & $0.0 \%$ & Pass: \\
\hline & IC25= & $>100 \%$ & & & \\
\hline
\end{tabular}

Text Paramelers

\section{Summary}

The reductions in survival and reproduction at the CTC are $८ 5 \%$ - Pass

Test for Homogeneity of Variance

Test Used: Bartlett's Test

$B=N A$

critical NA

NA

\section{Reproduction vs. Effluent Conc.}

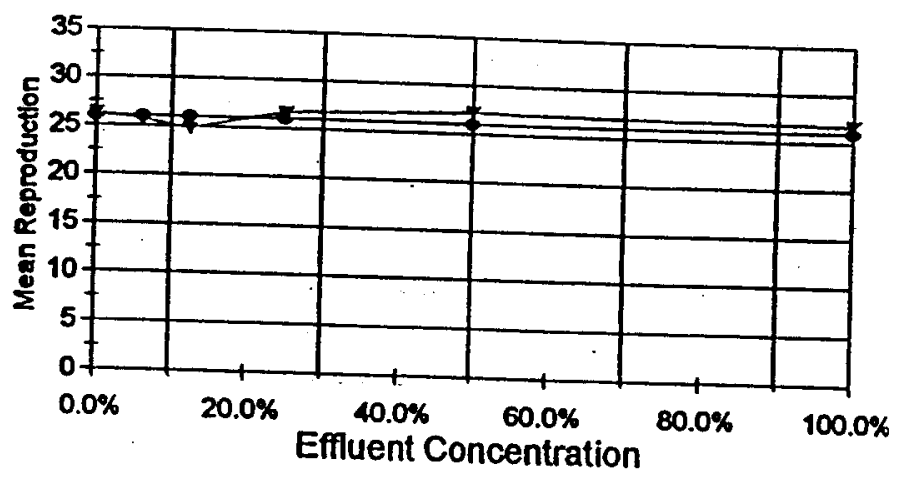

$\rightarrow$ Actual Reproduction $\rightarrow$ Predicted Reproduction 


\section{CHRONIC DEFINITIVE SURVIVAL AND REPRODUCTION TEST \\ Water Chemistry}

Client:

WSRC

Sample Identification:

Test Date:

A-01

February 26, 2002

pH (After Renewal)

\begin{tabular}{|l|r|r|r|r|r|r|}
\hline & \multicolumn{7}{|c|}{ Test Concentration } \\
\hline & control & $6.25 \%$ & $12.5 \%$ & $25 \%$ & $50 \%$ & $100 \%$ \\
\hline Initial & 7.75 & 7.46 & 7.12 & 7.07 & 7.04 & 7.00 \\
\hline Day 1 & 8.20 & 8.06 & 7.80 & 7.59 & 7.43 & 7.09 \\
\hline Day 2 & 7.11 & 6.93 & 6.85 & 7.93 & 7.07 & 7.24 \\
\hline Day 3 & 7.01 & 7.15 & 7.02 & 6.98 & 7.04 & 7.13 \\
\hline Day 4 & 7.02 & 7.23 & 7.19 & 7.11 & 7.05 & 6.98 \\
\hline Day 5 & 7.06 & 7.11 & 7.28 & 7.25 & 7.07 & 7.01 \\
\hline Day 6 & 7.04 & 6.95 & 6.85 & 6.94 & 6.96 & 7.11 \\
\hline
\end{tabular}

Dissolved Oxygen (After Renewal)

\begin{tabular}{|l|r|r|r|r|r|r|}
\hline & control & $6.25 \%$ & $12.5 \%$ & $25 \%$ & $50 \%$ & $100 \%$ \\
\hline Initial & 8.4 & 8.2 & 8.2 & 8.5 & 8.3 & 8.4 \\
\hline Day 1 & 8.4 & 8.2 & 8.2 & 8.3 & 8.3 & 8.3 \\
\hline Day 2 & 8.4 & 8.3 & 8.3 & 8.4 & 8.4 & 8.4 \\
\hline Day 3 & 8.4 & 8.2 & 8.2 & 8.3 & 8.4 & 8.4 \\
\hline Day 4 & 8.4 & 8.2 & 8.2 & 8.4 & 8.4 & 8.4 \\
\hline Day 5 & 8.3 & 8.4 & 8.3 & 8.3 & 8.4 & 8.4 \\
\hline Day 6 & 8.4 & 8.3 & 8.3 & 8.3 & 8.4 & 8.4 \\
\hline
\end{tabular}

Test Results Reviewed and Approved By:

pH (Before Renewal)
\begin{tabular}{|l|r|r|r|r|r|r|}
\hline \multicolumn{7}{|c|}{ Test Concentration } \\
\hline & control & $6.25 \%$ & $12.5 \%$ & $25 \%$ & $50 \%$ & $100 \%$ \\
\hline Day 1 & 8.25 & 8.09 & 7.92 & 7.83 & 7.86 & 7.66 \\
\hline Day 2 & 7.36 & 7.34 & 7.22 & 7.18 & 7.31 & 7.64 \\
\hline Day 3 & 7.34 & 7.26 & 7.23 & 7.24 & 7.30 & 7.43 \\
\hline Day 4 & 7.11 & 7.33 & 7.31 & 7.12 & 7.22 & 7.45 \\
\hline Day 5 & 7.09 & 7.18 & 7.26 & 7.24 & 7.36 & 7.38 \\
\hline Day 6 & 6.95 & 6.88 & 6.95 & 6.87 & 6.84 & 7.07 \\
\hline Final & 7.63 & 7.97 & & 7.38 & 7.43 & 7.47 \\
\hline
\end{tabular}

Dissolved Oxygen (Before Renewal)

\begin{tabular}{|l|r|r|r|r|r|r|}
\hline & \multicolumn{7}{|c|}{ Test Concentration } \\
\hline Day 1 & 8.3 & 8.3 & 8.3 & 8.4 & 8.4 & 8.4 \\
\hline Day 2 & 8.5 & 8.4 & 8.4 & 8.3 & 8.4 & 8.4 \\
\hline Day 3 & 8.5 & 8.4 & 8.4 & 8.4 & 8.5 & 8.5 \\
\hline Day 4 & 8.5 & 8.4 & 8.5 & 8.5 & 8.5 & 8.5 \\
\hline Day 5 & 8.4 & 8.4 & 8.4 & 8.3 & 8.3 & 8.3 \\
\hline Day 6 & 8.3 & 8.4 & 8.4 & 8.4 & 8.4 & 8.4 \\
\hline Final & 8.4 & 8.4 & & 8.3 & 8.4 & 8.4 \\
\hline
\end{tabular}

Temperature
\begin{tabular}{|l|r|}
\hline \multicolumn{2}{|c|}{ Incubator ${ }^{\circ} \mathrm{C}$} \\
\hline Initia & 25.5 \\
\hline Day 1 & 25.2 \\
\hline Day 2 & 25.2 \\
\hline Day 3 & 25.4 \\
\hline Day 4 & 25.4 \\
\hline Day 5 & 25.6 \\
\hline Day 6 & 25.4 \\
\hline Final & 25.0 \\
\hline
\end{tabular}




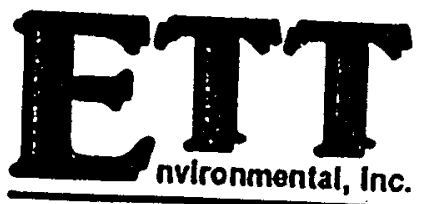

P.O. Box 16414, Greenville, SC 29606

(864) 877-6942 - FAX (864) 877-6938

4 Craftsman Court, Greer, SC 29650

\section{Day Chronic Definitive Survival and Reproduction Bioassay}

Method: EPA/600/4-91/002

Test Organism:

Daphnia ambigua

Facility: WSRC

Sample ID: A-11

Aiken County

ETT\#: T19344

Sample ID: February 26, 2002 


\section{CHRONIC DEFINITIVE SURVIVAL AND REPRODUCTION TEST Survival and Reproduction Results}

Client:

Sample Identification:

Test Date:

February 26, 2002

\begin{tabular}{|c|c|c|c|c|c|c|}
\hline \multicolumn{7}{|c|}{ Control } \\
\hline Reproduction at & Day 3/4 & Day 5 & Day 6 & Day 7 & Day 8 & Total \\
\hline 1 & 0 & 5 & 14 & $X$ & $X$ & 19 \\
\hline 2 & 7 & 0 & 10 & 11 & 0 & 28 \\
\hline 3 & 4 & 0 & 9 & 13 & 0 & 26 \\
\hline 4 & 0 & 5 & 10 & 0 & 14 & 29 \\
\hline 5 & 5 & 0 & 12 & $X$ & $X$ & 17 \\
\hline 6 & 5 & 0 & 14 & 15 & 0 & 34 \\
\hline 7 & 4 & 0 & 8 & 11 & 0 & 23 \\
\hline 8 & 6 & 0 & 13 & 12 & 0 & 31 \\
\hline 9 & 5 & 0 & 14 & 12 & 0 & 31 \\
\hline 10 & 4 & 0 & 10 & 11 & 0 & 25 \\
\hline
\end{tabular}

\section{Reproduction at}

$12.5 \%$

\begin{tabular}{|c|c|c|c|c|c|c|}
\hline Rep. & Day 3/4 & Day 5 & Day 6 & Day 7 & Day 8 & Total \\
\hline 1 & 7 & 0 & 13 & 0 & 14 & 34 \\
\hline 2 & 6 & 0 & 13 & 14 & 0 & 33 \\
\hline 3 & 0 & 5 & 13 & $X$ & $X$ & 18 \\
\hline 4 & 4 & 0 & 12 & $X$ & $X$ & 16 \\
\hline 5 & 0 & 6 & 13 & 0 & 11 & 30 \\
\hline 6 & $X$ & $X$ & $X$ & $X$ & $X$ & 0 \\
\hline 7 & 6 & 0 & 14 & 14 & 0 & 34 \\
\hline 8 & 6 & 0 & 12 & 13 & 0 & 31 \\
\hline 9 & 6 & 0 & 11 & 15 & 0 & 32 \\
\hline 10 & 0 & 6 & 14 & 0 & 12 & 32 \\
\hline
\end{tabular}

\begin{tabular}{|c|c|c|c|c|c|c|}
\hline Reproduction at & \multicolumn{5}{|c|}{$50 \%$} \\
\hline Rep. & Day 3/4 & Day 5 & Day 6 & Day 7 & Day & Total \\
\hline 1 & 0 & 6 & 12 & $X$ & $X$ & 18 \\
\hline 2 & 5 & 0 & 10 & 9 & 0 & 24 \\
\hline 3 & 0 & 6 & 14 & 0 & 15 & 35 \\
\hline 4 & 6 & 0 & 12 & 14 & 0 & 32 \\
\hline 5 & 1 & 0 & 12 & $X$ & $X$ & 13 \\
\hline 6 & 7 & 0 & 13 & 15 & 0 & 35 \\
\hline 7 & 0 & 7 & 10 & 0 & 10 & 27 \\
\hline 8 & 5 & 0 & 13 & $X$ & $X$ & 18 \\
\hline 9 & 0 & 5 & 11 & 0 & 12 & 28 \\
\hline 10 & 0 & 6 & 11 & 0 & 13 & 30 \\
\hline
\end{tabular}

County:

NPDES\#:

ETT \#:
Aiken

SC

T19344

\begin{tabular}{|c|c|c|c|c|c|c|}
\hline \multicolumn{6}{|c|}{ Reproduction at } \\
\hline Rep. & Day 3/4 & Day 5 & Day 6 & Day 7 & Day 8 & Total \\
\hline $\mathrm{I}$ & 0 & 4 & 7 & 0 & 14 & 25 \\
\hline 2 & 5 & 0 & 10 & 14 & 0 & 29 \\
\hline 3 & $\mathrm{~L}$ & $\mathrm{~L}$ & $\mathrm{~L}$ & $\mathrm{~L}$ & $\mathrm{~L}$ & $\mathrm{~L}$ \\
\hline 4 & $\mathrm{~L}$ & $\mathrm{~L}$ & $\mathrm{~L}$ & $\mathrm{~L}$ & $\mathrm{~L}$ & $\mathrm{~L}$ \\
\hline 5 & 7 & 0 & 13 & $\mathrm{X}$ & $\mathrm{X}$ & 20 \\
\hline 6 & 6 & 0 & 8 & 14 & 0 & 28 \\
\hline 7 & 6 & 0 & 14 & 14 & 0 & 34 \\
\hline 8 & 6 & 1 & 14 & 15 & 0 & 36 \\
\hline 9 & 6 & 0 & 14 & $\mathrm{X}$ & $\mathrm{X}$ & 20 \\
\hline 10 & 0 & 4 & 12 & $\mathrm{X}$ & $\mathrm{X}$ & 16 \\
\hline
\end{tabular}

\begin{tabular}{|c|c|c|c|c|c|c|}
\hline \multicolumn{6}{|c|}{ Reproduction at } \\
\hline Rep. & Day 3/4 & Day 5 & Day 6 & Day 7 & Day 8 & Total \\
\hline 1 & 8 & 0 & 14 & 14 & 0 & 36 \\
\hline 2 & 5 & 0 & 13 & 10 & 0 & 28 \\
\hline 3 & 0 & 5 & 10 & 0 & 7 & 22 \\
\hline 4 & 7 & 0 & 13 & 13 & 0 & 33 \\
\hline 5 & 7 & 0 & 12 & 0 & 10 & 29 \\
\hline 6 & 0 & 3 & 0 & 2 & 10 & 15 \\
\hline 7 & 6 & 0 & 12 & 13 & 0 & 31 \\
\hline 8 & 7 & 0 & 13 & 14 & 0 & 34 \\
\hline 9 & 7 & 0 & 13 & 14 & 0 & 34 \\
\hline 10 & 0 & 6 & 11 & 0 & 12 & 29 \\
\hline
\end{tabular}

\begin{tabular}{|c|c|c|c|c|c|c|}
\hline \multicolumn{7}{|c|}{ Reproduction at } \\
\hline Rep. & Day 3/4 & Day 5 & Day 6 & Day 7 & Day 8 & Toxal \\
\hline I & 6 & 0 & 14 & $\mathrm{X}$ & $\mathrm{X}$ & 20 \\
\hline 2 & 0 & 6 & 13 & 0 & 12 & 31 \\
\hline 3 & 0 & 6 & 13 & 0 & 10 & 29 \\
\hline 4 & $\mathrm{~L}$ & $\mathrm{~L}$ & $\mathrm{~L}$ & $\mathrm{~L}$ & $\mathrm{~L}$ & 0 \\
\hline 5 & 6 & 0 & 14 & 0 & 12 & 32 \\
\hline 6 & 5 & 0 & 15 & 0 & 12 & 32 \\
\hline 7 & 6 & 0 & 14 & 14 & 0 & 34 \\
\hline 8 & 4 & 0 & 12 & 10 & 0 & 26 \\
\hline 9 & 5 & 0 & 13 & $\mathrm{X}$ & $\mathrm{X}$ & 18 \\
\hline 10 & 0 & 4 & 10 & 0 & 13 & 27 \\
\hline
\end{tabular}




\section{CHRONIC DEFINITIVE SURVIVAL AND REPRODUCTION TEST \\ Statistical Analyses}

Client:

Sample Identification:

Test Date:
WSRC

A-11

February 26, 2002

\begin{tabular}{|c|c|c|}
\hline \multicolumn{3}{|l|}{ Test for Normality } \\
\hline Test Usod: Shapiro-Wilks & $\begin{array}{l}W= \\
\text { critical }\end{array}$ & $\begin{array}{l}\text { NA } \\
\text { NA }\end{array}$ \\
\hline $\mathbf{N A}$ & & \\
\hline
\end{tabular}

Test for Homogeneity of Variance

Test Used: Bartlett's Test

$B=N A$

critical NA

\begin{tabular}{|c|c|c|c|c|c|c|}
\hline \multicolumn{7}{|c|}{ Test for Differences in Reproduction } \\
\hline Teat Type Unod & & Non-Linear I & Ereston - I & $\begin{array}{l}\text { istic Mod } \\
\text { JMP Soft } \\
\text { Effibuent }\end{array}$ & & \\
\hline Ented & MHSF & $6.3 \%$ & $125 \%$ & $25.0 \%$ & $50.0 \%$ & $100 \%$ \\
\hline servived & $80 x$ & $100 x$ & $70 \%$ & $100 \%$ & $70 \%$ & $70 \%$ \\
\hline Irocicted & 100x. & $100 \%$ & $100 \%$ & $100 x$ & $100 \%$ & $100 \%$ \\
\hline Reproduction & 26.3 & 26.0 & 26.0 & 29.1 & 26.0 & 27.7 \\
\hline Prodicted & 26.6 & 26.6 & 26.6 & 26.6 & 26.6 & 26.6 \\
\hline Varimose & 20.57 & 50.00 & 125.56 & 40.54 & 57.7 & 30.75 \\
\hline \multirow{2}{*}{\multicolumn{4}{|c|}{ Teat Purnmeters }} & & $\mathbf{M S D}=$ & 7.75 \\
\hline & & & & & & \\
\hline & dpper & bets & samman & \multicolumn{2}{|c|}{$\begin{array}{l}\% \text { Redection } \\
\text { CTO- } \% .1 \%\end{array}$} & Pasaral \\
\hline Sunvint & 3.00 & -200.00 & 0.00 & \multicolumn{2}{|c|}{$0.0 \%$} & Pex \\
\hline Reproduction & 299 & -5.89 & 26.70 & \multicolumn{2}{|c|}{$0.0 \%$} & Pan: \\
\hline & $\mathrm{IC25}=$ & $>100 \%$ & & & & \\
\hline
\end{tabular}

Reproduction vs. Effluent Conc.

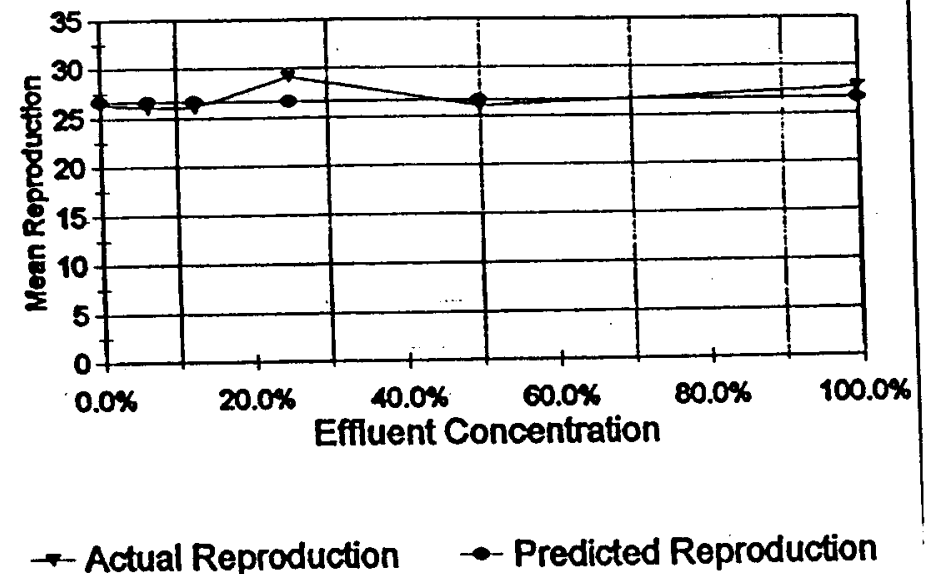

Actual Reproduction $\rightarrow$ Predicted Reproduction

Summary

The reductions in survival and reproduction at the CTC are $<25 \%$ - Pass 


\section{CHRONIC DEFINITIVE SURVIVAL AND REPRODUCTION TEST \\ Water Chemistry}

Client: WSRC

Sample Identification:

A-11

Test Date:

February 26, 2002

pH (After Renewal)

\begin{tabular}{|l|r|r|r|r|r|r|}
\hline & Test Concentration \\
\hline Initial & 7.75 & 7.64 & 7.41 & 8.38 & 7.32 & 7.29 \\
\hline Day 1 & 8.20 & 7.12 & 6.95 & 6.97 & 7.94 & 7.02 \\
\hline Day 2 & 7.11 & 6.70 & 6.63 & 6.65 & 6.72 & 6.73 \\
\hline Day 3 & 7.01 & 7.21 & 7.10 & 7.10 & 7.09 & 7.16 \\
\hline Day 4 & 7.02 & 6.99 & 7.23 & 7.24 & 7.21 & 7.19 \\
\hline Day 5 & 7.06 & 7.01 & 7.07 & 7.22 & 7.28 & 7.33 \\
\hline Day 6 & 7.01 & 7.05 & 7.06 & 6.91 & 6.94 & 7.09 \\
\hline
\end{tabular}

Dissolved Oxygen (After Renewal)

\begin{tabular}{|l|r|r|r|r|r|r|}
\hline & \multicolumn{7}{|c|}{ Test Concentration } \\
\hline Initial & 8.4 & 8.2 & 8.3 & 8.2 & 8.2 & 8.2 \\
\hline Day 1 & 8.4 & 8.3 & 8.3 & 8.3 & 8.4 & 8.4 \\
\hline Day 2 & 8.4 & 8.3 & 8.3 & 8.4 & 8.4 & 8.5 \\
\hline Day 3 & 8.4 & 8.4 & 8.4 & 8.5 & 8.5 & 8.6 \\
\hline Day 4 & 8.4 & 8.4 & 8.4 & 8.4 & 8.4 & 8.4 \\
\hline Day 5 & 8.3 & 8.3 & 8.3 & 8.4 & 8.4 & 8.4 \\
\hline Day 6 & 8.4 & 8.5 & 8.5 & 8.5 & 8.5 & 8.5 \\
\hline
\end{tabular}

pH (Before Renewal)

\begin{tabular}{|l|r|r|r|r|r|r|}
\hline & control & $6.25 \%$ & $12.5 \%$ & $25 \%$ & $50 \%$ & $100 \%$ \\
\hline Day 1 & 8.25 & 7.61 & 7.54 & 7.55 & 7.44 & 7.45 \\
\hline Day 2 & 7.36 & 7.30 & 7.12 & 7.11 & 7.14 & 7.20 \\
\hline Day 3 & 7.34 & 7.43 & 7.37 & 7.33 & 7.30 & 7.31 \\
\hline Day 4 & 7.11 & 7.34 & 7.31 & 7.12 & 7.11 & 7.02 \\
\hline Day 5 & 7.09 & 7.11 & 7.29 & 7.34 & 7.25 & 7.41 \\
\hline Day 6 & 6.95 & 6.81 & 6.84 & 8.86 & 8.90 & 7.01 \\
\hline Final & 7.63 & 7.49 & 7.06 & 7.24 & 7.18 & 7.17 \\
\hline
\end{tabular}

Dissolved Oxygen (Before Renewal)

\begin{tabular}{|l|r|r|r|r|r|r|}
\hline \multicolumn{7}{|c|}{ Test Concentration } \\
\hline & control & $6.25 \%$ & $12.5 \%$ & $25 \%$ & $50 \%$ & $100 \%$ \\
\hline Day 1 & 8.4 & 8.3 & 8.3 & 8.1 & 8.4 & 8.4 \\
\hline Day 2 & 8.5 & 8.4 & 8.4 & 8.4 & 8.5 & 8.4 \\
\hline Day 3 & 8.5 & 8.3 & 8.4 & 8.4 & 8.5 & 8.5 \\
\hline Day 4 & 8.5 & 8.4 & 8.4 & 8.4 & 8.5 & 8.5 \\
\hline Day 5 & 8.4 & 8.4 & 8.4 & 8.4 & 8.4 & 8.3 \\
\hline Day 6 & 8.3 & 8.5 & 8.5 & 8.6 & 8.6 & 8.6 \\
\hline Final & 8.4 & 8.5 & 8.6 & 8.6 & 8.5 & 8.5 \\
\hline
\end{tabular}

Test Results Reviewed and Approved By:

Temperature
\begin{tabular}{|l|r|}
\hline \multicolumn{1}{|c|}{ Incubator ${ }^{\circ} \mathrm{C}$} \\
\hline Initial & 25.5 \\
\hline Day 1 & 25.2 \\
\hline Day 2 & 25.2 \\
\hline Day 3 & 25.4 \\
\hline Day 4 & 25.4 \\
\hline Day 5 & 25.6 \\
\hline Day 6 & 25.4 \\
\hline Final & 25.0 \\
\hline
\end{tabular}




\section{Day Chronic Definitive Survival and Reproduction Bioassay} Method: EPA/600/4-91/002

Test Organism:

Daphnia ambigua

Facility: WSRC

Sample ID: G-10

Aiken County

ETT\#: T19345

Sample ID: February 26, 2002 


\section{CHRONIC DEFINITIVE SURVIVAL AND REPRODUCTION TEST}

\section{Survival and Reproduction Results}

Client:

Sample Identification:

Test Date:

WSRC

G-10

February 26, 2002
County:

NPDES\#:

ETT \#:
Aiken

SC

T19345

\begin{tabular}{|c|c|c|c|c|c|c|}
\hline \multicolumn{7}{|c|}{ Reproduction at } \\
\hline Rep. & Day 3/4 & Day 5 & Day 6 & Day 7 & Day 8 & Total \\
\hline 1 & 0 & 5 & 14 & $X$ & $X$ & 19 \\
\hline 2 & 7 & 0 & 10 & 11 & 0 & 28 \\
\hline 3 & 4 & 0 & 9 & 13 & 0 & 26 \\
\hline 4 & 0 & 5 & 10 & 0 & 14 & 29 \\
\hline 5 & 5 & 0 & 12 & $X$ & $X$ & 17 \\
\hline 6 & 5 & 0 & 14 & 15 & 0 & 34 \\
\hline 7 & 4 & 0 & 8 & 11 & 0 & 23 \\
\hline 8 & 6 & 0 & 13 & 12 & 0 & 31 \\
\hline 9 & 5 & 0 & 14 & 12 & 0 & 31 \\
\hline 10 & 4 & 0 & 10 & 11 & 0 & 25 \\
\hline
\end{tabular}

\begin{tabular}{|c|c|c|c|c|c|c|}
\hline \multicolumn{2}{|c|}{ Reproduction at } & \multicolumn{2}{c|}{$6.25 \%$} & \multicolumn{2}{c|}{ Concentration } \\
\hline Rep. & Day 3/4 & Day 5 & Day 6 & Day 7 & Day 8 & Total \\
\hline 1 & 0 & 0 & 12 & 14 & 0 & 26 \\
\hline 2 & 4 & 0 & 13 & 15 & 0 & 32 \\
\hline 3 & 3 & 0 & 12 & 13 & 0 & 28 \\
\hline 4 & 5 & 0 & 12 & 14 & 0 & 31 \\
\hline 5 & 5 & 0 & $X$ & $X$ & $X$ & 5 \\
\hline 6 & 6 & 0 & 14 & 13 & 0 & 33 \\
\hline 7 & 0 & 3 & 12 & 0 & 14 & 29 \\
\hline 8 & 0 & 5 & 12 & 0 & 13 & 30 \\
\hline 9 & 5 & 0 & 8 & 12 & 0 & 25 \\
\hline 10 & 0 & 5 & 15 & 0 & $X$ & 20 \\
\hline
\end{tabular}

\begin{tabular}{|c|c|c|c|c|c|c|}
\hline \multicolumn{2}{|c|}{ Reproduction at } & \multicolumn{4}{c|}{$12.5 \%$} \\
\hline Rep. & Day 3/4 & Day 5 & Day 6 & Day 7 & Day 8 & Total \\
\hline 1 & 4 & 0 & 9 & 10 & 0 & 23 \\
\hline 2 & 0 & 4 & 9 & 0 & 12 & 25 \\
\hline 3 & 0 & 6 & 10 & $\mathrm{X}$ & $\mathrm{X}$ & 16 \\
\hline 4 & $\mathrm{X}$ & $\mathrm{X}$ & $\mathrm{X}$ & $\mathrm{X}$ & $\mathrm{X}$ & 0 \\
\hline 5 & 3 & 0 & 10 & 10 & 0 & 23 \\
\hline 6 & 5 & 0 & 14 & 16 & 0 & 35 \\
\hline 7 & 0 & 5 & $\mathrm{X}$ & $\mathrm{X}$ & $\mathrm{X}$ & 5 \\
\hline 8 & 0 & 7 & 15 & 0 & 13 & 35 \\
\hline 9 & floater & $\mathrm{L}$ & $\mathrm{L}$ & $\mathrm{L}$ & $\mathrm{L}$ & $\mathrm{L}$ \\
\hline 10 & 0 & 7 & 0 & 11 & 11 & 29 \\
\hline
\end{tabular}

\begin{tabular}{|c|c|c|c|c|c|c|}
\hline \multicolumn{3}{|c|}{ Reproduction at } & \multicolumn{3}{c|}{$25 \%$} & \multicolumn{2}{c|}{ Concentration } \\
\hline Rep. & Day 3/4 & Day 5 & Day 6 & Day 7 & Day 8 & Total \\
\hline 1 & 4 & 7 & 0 & 10 & 0 & 21 \\
\hline 2 & 6 & 0 & 14 & 14 & 0 & 34 \\
\hline 3 & floater & $\mathrm{L}$ & $\mathrm{L}$ & $\mathrm{L}$ & $\mathrm{L}$ & $\mathrm{L}$ \\
\hline 4 & floater & $\mathrm{L}$ & $\mathrm{L}$ & $\mathrm{L}$ & $\mathrm{L}$ & $\mathrm{L}$ \\
\hline 5 & 5 & 0 & 13 & 16 & 0 & 34 \\
\hline 6 & 0 & 4 & $\mathrm{X}$ & $\mathrm{X}$ & $\mathrm{X}$ & 4 \\
\hline 7 & 4 & 0 & $\mathrm{X}$ & $\mathrm{X}$ & $\mathrm{X}$ & 4 \\
\hline 8 & 0 & 6 & 13 & 0 & 13 & 32 \\
\hline 9 & floater & $\mathrm{L}$ & $\mathrm{L}$ & $\mathrm{L}$ & $\mathrm{L}$ & $\mathrm{L}$ \\
\hline 10 & 0 & 7 & 13 & 0 & 14 & 34 \\
\hline
\end{tabular}

\begin{tabular}{|c|c|c|c|c|c|c|}
\hline \multicolumn{2}{|c|}{ Reproduction at } & \multicolumn{3}{c|}{$50 \%$} & \multicolumn{3}{c|}{ Concentration } \\
\hline Rep. & Day 3/4 & Day 5 & Day 6 & Day 7 & Day 8 & Tocal \\
\hline 1 & 0 & 7 & $X$ & $X$ & $X$ & 7 \\
\hline 2 & 6 & 0 & 15 & 16 & 0 & 37 \\
\hline 3 & lost & $\mathrm{L}$ & $\mathrm{L}$ & $\mathrm{L}$ & $\mathrm{L}$ & $\mathrm{L}$ \\
\hline 4 & 7 & 0 & 14 & 13 & 0 & 34 \\
\hline 5 & 5 & 0 & 10 & 13 & 0 & 28 \\
\hline 6 & 5 & 0 & 12 & 12 & 0 & 29 \\
\hline 7 & 0 & 6 & 12 & 0 & 15 & 33 \\
\hline 8 & 0 & 4 & 15 & 0 & 13 & 32 \\
\hline 9 & 3 & 0 & 14 & 15 & 0 & 32 \\
\hline 10 & 0 & 4 & 10 & 0 & 10 & 24 \\
\hline
\end{tabular}

\begin{tabular}{|c|c|c|c|c|c|c}
\hline \multicolumn{2}{|c|}{ Reproduction at } & \multicolumn{2}{c|}{$100 \%$} & \multicolumn{2}{c}{ Concentration } \\
\hline Rep. & Day 3/4 & Day 5 & Day6 & Day 7 & Day 8 & Total \\
\hline 1 & 3 & 0 & 11 & 13 & 0 & 27 \\
\hline 2 & 5 & 0 & 12 & 14 & 0 & 31 \\
\hline 3 & 0 & 0 & 13 & 10 & 0 & 23 \\
\hline 4 & 4 & 0 & 10 & 15 & 0 & 29 \\
\hline 5 & 1 & 2 & 12 & 13 & 0 & 28 \\
\hline 6 & 1 & 0 & 13 & 13 & 0 & 27 \\
\hline 7 & 0 & 4 & 11 & 0 & 14 & 29 \\
\hline 8 & 4 & 3 & 13 & 7 & 0 & 27 \\
\hline 9 & 6 & 0 & 14 & 15 & 0 & 35 \\
\hline 10 & 0 & 4 & 12 & 0 & 12 & 28 \\
\hline
\end{tabular}




\section{CHRONIC DEFINITIVE SURVIVAL AND REPRODUCTION TEST \\ Statistical Analyses}

Client: $\quad$ WSRC

Sample Identification: $\quad$ G-10

Test Date:

February 26, 2002

\section{Test for Normality}

Test Used: Shapiro-Wilks

$\begin{array}{ll}W= & N A \\ \text { critical } & N A\end{array}$

NA
Test for Homogeneity of Variance

Test Used: Bartlett's Test

$B=N A$

critical NA
Test for Differences in Reproduction

\begin{tabular}{|c|c|c|c|c|c|c|}
\hline \multicolumn{2}{|c|}{ Tes Type Used: } & \multicolumn{5}{|c|}{ Non-Linear Regression - Logistic Model } \\
\hline & & \multicolumn{5}{|c|}{ Calculaled Using JMP Software } \\
\hline & & \multicolumn{5}{|c|}{$\%$ Emluent } \\
\hline Enect & MHSF & $6.3 \%$ & $12.5 \%$ & $25,0 \%$ & $50.0 \%$ & $100 \%$ \\
\hline Survival & $80 \%$ & $100 \%$ & $67 \%$ & $71 \%$ & $89 \%$ & $100 \%$ \\
\hline Iredicied & $100 \%$ & $100 \%$ & $100 \%$ & $100 \%$ & $100 \%$ & $100 \%$ \\
\hline Reproduction & 26.3 & 25.9 & 21.2 & 23.3 & 28.4] & 28.4 \\
\hline Fredicted & 25.7 & 25.7 & 25.7 & 25.7 & 25.7 & 25.7 \\
\hline Veriance & 29.57 & 68.54 & 150.19 & 194.90 & 78.78 & 9.60 \\
\hline \multirow{2}{*}{\multicolumn{4}{|c|}{ Test Parameter: }} & & MSD= & 9.29 \\
\hline & & & & & & \\
\hline & alpha & beta & gamma & \multicolumn{2}{|c|}{$\begin{array}{c}\text { x Reduction } \\
\text { aCTC }\end{array}$} & Passifail \\
\hline Survivel & 3.00 & -200.00 & 0.00 & \multicolumn{2}{|c|}{$0.0 \%$} & Pess \\
\hline Reproduction & 4.78 & -235.00 & 25.70 & \multicolumn{2}{|c|}{$0.0 \%$} & Pass \\
\hline
\end{tabular}

\section{Reproduction vs. Effluent Conc.}

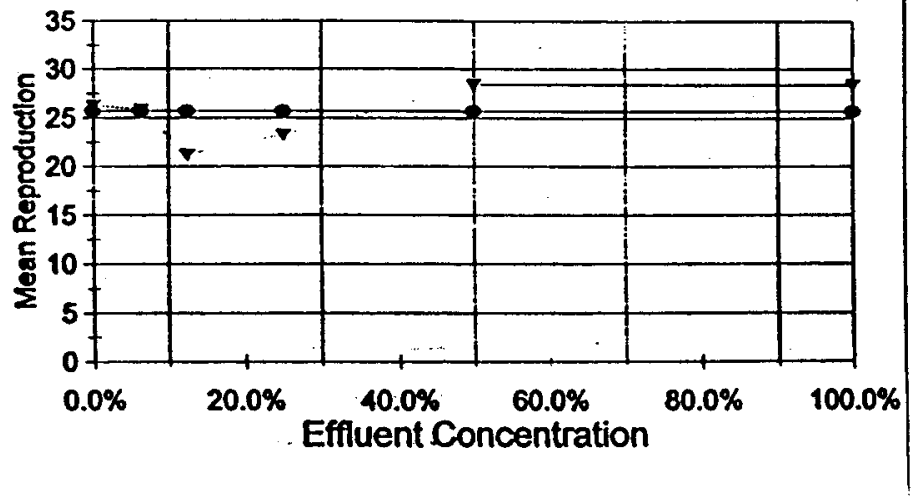

$\rightarrow$ Actual Reproduction $\rightarrow$ Predicted Reproduction

Summary

The reductions in survival and reproduction at the $\mathrm{CTC}$ are $<25 \%-\mathrm{Pass}$ 


\section{CHRONIC DEFINITIVE SURVIVAL AND REPRODUCTION TEST \\ Water Chemistry}

Client: $\quad$ WSRC

Sample Identification:

G-10

Test Date:

February 26, 2002

pH (After Renewal)

\begin{tabular}{|l|r|r|r|r|r|r|}
\hline \multicolumn{7}{|c|}{ Test Concentration } \\
\hline & control & $6.25 \%$ & $12.5 \%$ & $25 \%$ & $50 \%$ & $100 \%$ \\
\hline Initial & 6.92 & 7.17 & 7.36 & 7.67 & 7.72 & 8.02 \\
\hline Day 1 & 7.79 & 7.50 & 7.56 & 7.57 & 7.72 & 8.20 \\
\hline Day 2 & 7.10 & 7.14 & 7.34 & 7.59 & 7.85 & 8.07 \\
\hline Day 3 & 7.63 & 7.53 & 7.38 & 7.64 & 7.72 & 7.89 \\
\hline Day 4 & & 7.73 & 7.89 & 7.88 & 7.92 & 8.02 \\
\hline Day 5 & 7.17 & 7.33 & 7.56 & 7.78 & 7.99 & 8.01 \\
\hline Day 6 & 7.03 & 7.10 & 7.19 & 7.26 & 7.66 & 7.78 \\
\hline
\end{tabular}

Dissolved Oxygen (After Renewal)

\begin{tabular}{|l|r|r|r|r|r|r|}
\hline & control & $6.25 \%$ & $12.5 \%$ & $25 \%$ & $50 \%$ & $100 \%$ \\
\hline Initial & 8.2 & 8.2 & 8.3 & 8.2 & 8.2 & 8.3 \\
\hline Day 1 & 8.3 & 8.2 & 8.2 & 8.2 & 8.2 & 8.2 \\
\hline Day 2 & 8.4 & 8.4 & 8.4 & 8.5 & 8.5 & 8.5 \\
\hline Day 3 & 8.4 & 8.3 & 8.3 & 8.4 & 8.4 & 8.5 \\
\hline Day 4 & & 8.4 & 8.4 & 8.4 & 8.4 & 8.4 \\
\hline Day 5 & 8.4 & 8.3 & 8.3 & 8.4 & 8.4 & 8.4 \\
\hline Day 6 & 8.4 & 8.4 & 8.4 & 8.4 & 8.4 & 8.4 \\
\hline
\end{tabular}

pH (Before Renewal)

\begin{tabular}{|l|r|r|r|r|r|r|}
\hline & control & $6.25 \%$ & $12.5 \%$ & $25 \%$ & $50 \%$ & $100 \%$ \\
\hline Day 1 & 8.34 & 7.78 & 7.66 & 7.73 & 7.81 & 8.22 \\
\hline Day 2 & 7.47 & 7.61 & 7.65 & 7.79 & 8.08 & 8.38 \\
\hline Day 3 & 7.40 & 7.81 & 7.89 & 7.93 & 7.99 & 8.05 \\
\hline Day 4 & 7.53 & 7.72 & 7.74 & 7.92 & 8.09 & 8.11 \\
\hline Day 5 & 7.31 & 7.54 & 7.66 & 7.89 & 7.99 & 8.10 \\
\hline Day 6 & 6.87 & 6.98 & 7.15 & 7.21 & 7.54 & 7.66 \\
\hline Final & 7.25 & 7.29 & 7.34 & 7.44 & 7.52 & 7.72 \\
\hline
\end{tabular}

Dissolved Oxygen (Before Renewal)

\begin{tabular}{|l|r|r|r|r|r|r}
\hline & control & $6.25 \%$ & $12.5 \%$ & $25 \%$ & $50 \%$ & $100 \%$ \\
\hline Day 1 & 8.3 & 8.4 & 8.4 & 8.4 & 8.4 & 8.5 \\
\hline Day 2 & 8.5 & 8.4 & 8.4 & 8.5 & 8.5 & 8.6 \\
\hline Day 3 & 8.5 & 8.4 & 8.4 & 8.4 & 8.4 & 8.6 \\
\hline Day 4 & & 8.6 & 8.3 & 8.5 & 8.6 & 8.6 \\
\hline Day 5 & 8.4 & 8.4 & 8.4 & 8.4 & 8.4 & 8.4 \\
\hline Day 6 & 8.3 & 8.4 & 8.4 & 8.4 & 8.5 & 8.5 \\
\hline Final & 8.4 & 8.4 & 8.4 & 8.4 & 8.5 & 8.6 \\
\hline
\end{tabular}

Temperature
\begin{tabular}{|l|r|}
\hline \multicolumn{1}{|c|}{ Incubator ${ }^{\circ} \mathrm{C}$} \\
\hline Initial & 25.5 \\
\hline Day 1 & 25.2 \\
\hline Day 2 & 25.2 \\
\hline Day 3 & 25.4 \\
\hline Day 4 & 25.4 \\
\hline Day 5 & 25.6 \\
\hline Day 6 & 25.4 \\
\hline Final & 25.0 \\
\hline
\end{tabular}




\section{Day Chronic Definitive Survival and Reproduction Bioassay \\ Method: EPA/600/4-91/002}

Test Organism:

Daphnia ambigua

Facility: WSRC

Sample ID: A-01

Aiken County

ETT\#: 19621

Sample ID: April 9, 2002 


\section{CHRONIC DEFINITIVE SURVIVAL AND REPRODUCTION TEST}

Survival and Reproduction Results

\section{Client: $\quad$ WSRC}

Sample Identification:

Test Date:

April 9, 2002

$\begin{array}{ll}\text { County: } & \text { Aiken } \\ \text { NPDES\#: } & \text { SC } \\ \text { ETT \#: } & 19621\end{array}$

\begin{tabular}{|c|c|c|c|c|c|c|}
\hline \multicolumn{2}{|c|}{ Reproduction at } & \multicolumn{6}{c|}{$6.2 \%$} \\
\hline Rep. & Day 3/4 & Day 5 & Day 6 & Day 7 & Day 8 & Total \\
\hline 1 & 4 & 0 & 8 & 0 & 12 & 24 \\
\hline 2 & 6 & 0 & 10 & 0 & 11 & 27 \\
\hline 3 & 5 & 7 & 1 & 0 & 16 & 29 \\
\hline 4 & 4 & 0 & 10 & $X$ & $X$ & 14 \\
\hline 5 & 5 & 0 & 12 & 0 & 15 & 32 \\
\hline 6 & 5 & 0 & 13 & 0 & 15 & 33 \\
\hline 7 & 4 & $X$ & $X$ & $X$ & $X$ & 4 \\
\hline 8 & 0 & 3 & 10 & 0 & 15 & 28 \\
\hline 9 & 0 & 5 & 11 & 0 & 12 & 28 \\
\hline 10 & 0 & 7 & 13 & 0 & 12 & 32 \\
\hline
\end{tabular}

\begin{tabular}{|c|c|c|c|c|c|c}
\hline \multicolumn{3}{|c|}{ Reproduction at } & \multicolumn{2}{c}{$25 \%$} \\
\hline Rep. & Day 3/4 & Day 5 & Day 6 & Day 7 & Day 8 & Toall \\
\hline 1 & 4 & 0 & 10 & 0 & 14 & 28 \\
\hline 2 & 5 & 0 & 12 & 0 & 16 & 33 \\
\hline 3 & 6 & 0 & 10 & 0 & 15 & 31 \\
\hline 4 & 4 & 0 & 11 & 0 & 17 & 32 \\
\hline 5 & 7 & 0 & 11 & 0 & 16 & 34 \\
\hline 6 & 5 & 0 & 11 & 0 & 14 & 30 \\
\hline 7 & 3 & 0 & 10 & 0 & 13 & 26 \\
\hline 8 & 0 & $X$ & $X$ & $X$ & $X$ & 0 \\
\hline 9 & 0 & 3 & 10 & 0 & 11 & 24 \\
\hline 10 & 0 & 5 & 7 & 0 & 14 & 26 \\
\hline
\end{tabular}

\begin{tabular}{|c|c|c|c|c|c|c}
\hline Reproduction at & \multicolumn{4}{c}{$100 \%$} & \multicolumn{2}{c}{ Concentration } \\
\hline Rep. & Day 3/4 & Day 5 & Day 6 & Day 7 & Day & Tolal \\
\hline 1 & 4 & 0 & 4 & 0 & 12 & 20 \\
\hline 2 & 3 & 0 & 8 & 0 & 11 & 22 \\
\hline 3 & 5 & 0 & 3 & 0 & 6 & 14 \\
\hline 4 & 6 & 0 & 7 & 0 & 18 & 31 \\
\hline 5 & 4 & 0 & 10 & 0 & 16 & 30 \\
\hline 6 & 4 & 0 & 12 & 0 & 17 & 33 \\
\hline 7 & 0 & 7 & 10 & 0 & 15 & 32 \\
\hline 8 & 0 & 3 & 9 & 0 & 11 & 23 \\
\hline 9 & 0 & 6 & 12 & 0 & 17 & 35 \\
\hline 10 & 4 & 0 & 12 & 0 & 15 & 31 \\
\hline
\end{tabular}

\begin{tabular}{|c|c|c|c|c|c|c|}
\hline \multicolumn{2}{|c|}{ Reproduction at } & \multicolumn{4}{c|}{$12.5 \%$} \\
\hline Rep. & Day 3/4 & Day 5 & Day 6 & Day 7 & Day 8 & Total \\
\hline 1 & 5 & 7 & 0 & 16 & 0 & 28 \\
\hline 2 & 6 & 0 & 10 & 0 & 12 & 28 \\
\hline 3 & 4 & 0 & 8 & 0 & 16 & 28 \\
\hline 4 & 4 & 0 & 11 & 0 & 13 & 28 \\
\hline 5 & 5 & 0 & 9 & 0 & 14 & 28 \\
\hline 6 & 3 & 0 & 10 & $X$ & $X$ & 13 \\
\hline 7 & 0 & 7 & 8 & 0 & 11 & 26 \\
\hline 8 & 0 & 5 & 10 & 0 & 0 & 15 \\
\hline 9 & 0 & 5 & 8 & 0 & 11 & 24 \\
\hline 10 & 0 & 5 & 9 & 0 & 15 & 29 \\
\hline
\end{tabular}

\begin{tabular}{|c|c|c|c|c|c|c|}
\hline \multicolumn{2}{|c|}{ Reproduction at } & \multicolumn{3}{c|}{$50 \%$} & \multicolumn{3}{c|}{ Concentration } \\
\hline Rep. & Day 3/4 & Day 5 & Day 6 & Day 7 & Day 8 & Tolal \\
\hline 1 & 4 & 0 & 10 & 0 & 14 & 28 \\
\hline 2 & 5 & 0 & 8 & 0 & 16 & 29 \\
\hline 3 & 5 & 0 & 0 & 0 & 16 & 21 \\
\hline 4 & 6 & 0 & 10 & 0 & 15 & 31 \\
\hline 5 & 5 & 0 & 11 & 0 & 16 & 32 \\
\hline 6 & 6 & 0 & 8 & 0 & 12 & 26 \\
\hline 7 & 0 & 5 & 10 & 0 & 16 & 31 \\
\hline 8 & 0 & 6 & 9 & 0 & 10 & 25 \\
\hline 9 & 0 & 3 & 11 & 0 & 11 & 25 \\
\hline 10 & 5 & 0 & 9 & 0 & 13 & 27 \\
\hline
\end{tabular}

\begin{tabular}{|c|c|c|c|c|c|c|}
\hline \multicolumn{7}{|c|}{ Meproduction at } \\
\hline Rep. & Day 3/4 & Day 5 & Day 6 & Day 7 & Day 8 & Total \\
\hline 1 & 6 & 0 & 4 & 0 & 12 & 22 \\
\hline 2 & 5 & 0 & 3 & 7 & 0 & 15 \\
\hline 3 & 6 & 0 & 6 & 14 & 0 & 26 \\
\hline 4 & 4 & 0 & 9 & 14 & 0 & 27 \\
\hline 5 & 7 & 0 & 10 & 13 & 0 & 30 \\
\hline 6 & 6 & 0 & 11 & 15 & 0 & 32 \\
\hline 7 & 0 & 6 & 10 & 0 & 0 & 16 \\
\hline 8 & 0 & 4 & 12 & 0 & 11 & 27 \\
\hline 9 & 0 & 8 & 12 & 0 & 12 & 32 \\
\hline 10 & 0 & 5 & 8 & 0 & 13 & 26 \\
\hline
\end{tabular}




\section{Day Chronic Definitive Survival and Reproduction Bioassay \\ Method: EPA/600/4-91/002}

Test Organism:

Daphnia ambigua

Facility: WSRC

Sample ID: A-11

$\cdots$

Aiken County

ETT\#: 19622

Sample ID: April 9, 2002 


\section{CHRONIC DEFINITIVE SURVIVAL AND REPRODUCTION TEST Statistical Analyses}

Client:

Sample Identification:

Test Date:
WSRC

$$
\text { A-01 }
$$

April 9,2002

\section{Test for Normality}

Test Used: Kolmogorov D

NA

$\begin{array}{ll}D= & \text { NA } \\ \text { critical } & \text { NA }\end{array}$

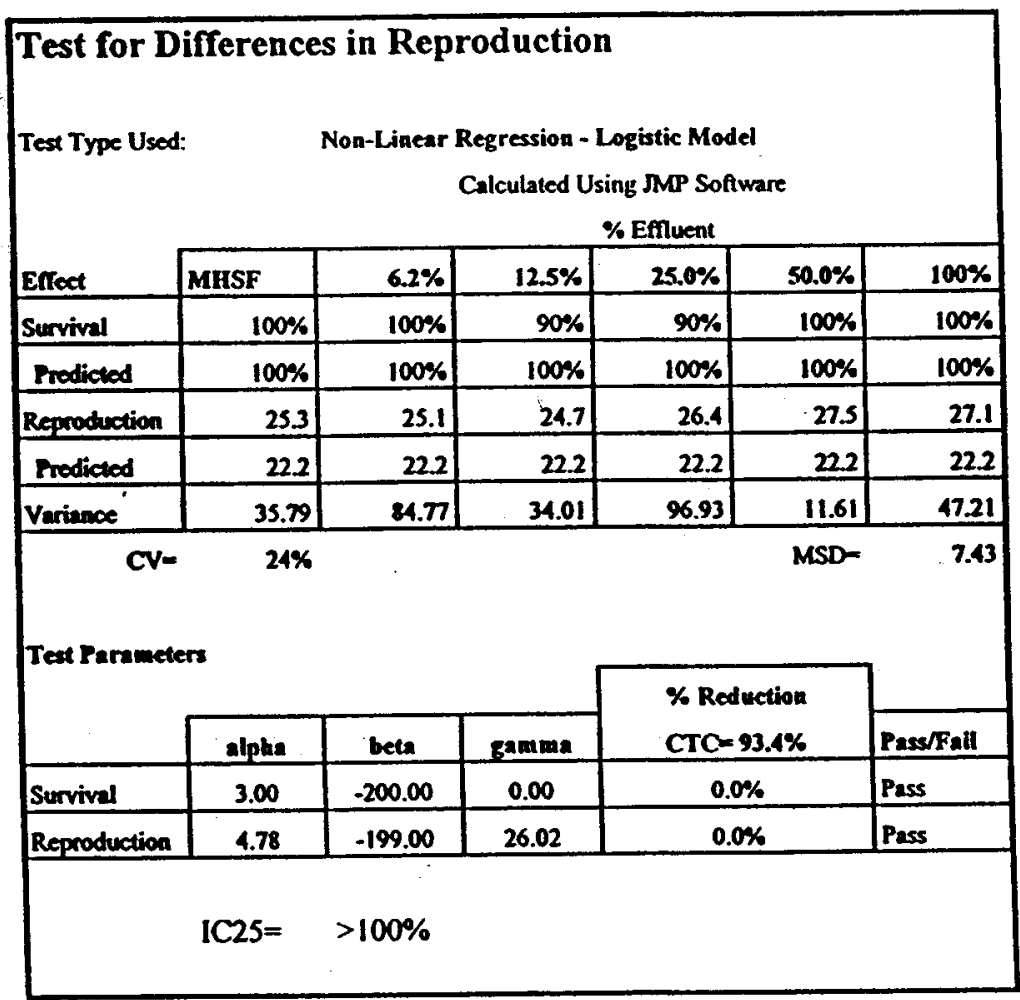

Test for Homogeneity of Variance

Test Used: Bartlett's Test

$B=N A$

critical NA

NA

\section{Reproduction vs. Effluent Conc.}

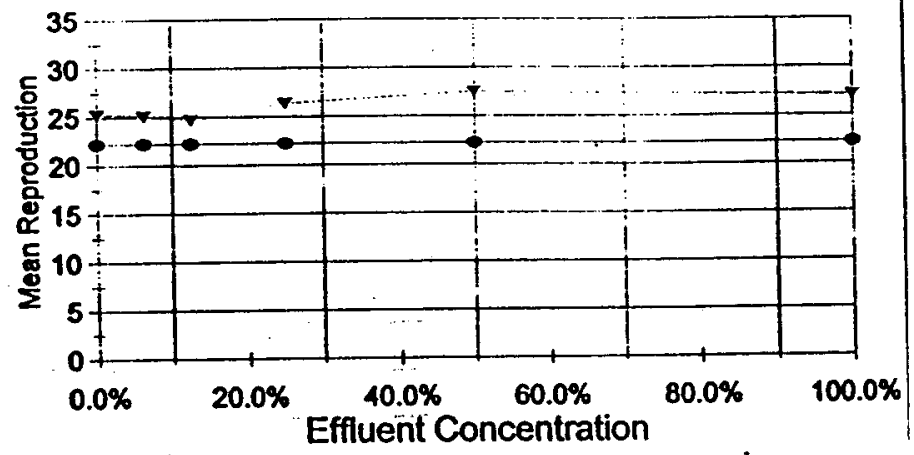

Actual Reproduction

\section{Summary}

The reductions in survival and reproduction at the CTC are $<25 \%$ - Pass 


\section{CHRONIC DEFINITIVE SURVIVAL AND REPRODUCTION TEST}

Survival and Reproduction Results

Client: WSRC

Sample Identification:

Test Date:

April 9, 2002

$\begin{array}{ll}\text { County: } & \text { Aiken } \\ \text { NPDESH: } & \text { SC } \\ \text { ETT \#: } & 19622\end{array}$

\begin{tabular}{|c|c|c|c|c|c|c|}
\hline \multicolumn{2}{|c|}{ Reproduction at } & \multicolumn{6}{|c|}{$6.2 \%$} \\
\hline Rep. & Day 3/4 & Day 5 & Day 6 & Day 7 & Day 8 & Total \\
\hline 1 & 6 & 7 & 0 & 14 & 0 & 27 \\
\hline 2 & 4 & 0 & 10 & 13 & 0 & 27 \\
\hline 3 & 4 & 0 & 12 & 0 & 16 & 32 \\
\hline 4 & 4 & 0 & 11 & 0 & 12 & 27 \\
\hline 5 & 4 & 0 & 5 & 0 & 6 & 15 \\
\hline 6 & 2 & 0 & 9 & 0 & 15 & 26 \\
\hline 7 & 0 & 5 & 9 & 0 & 15 & 29 \\
\hline 8 & 5 & 0 & 6 & 13 & 0 & 24 \\
\hline 9 & 0 & 5 & 8 & 0 & 16 & 29 \\
\hline 10 & 3 & 0 & 10 & 0 & 12 & 25 \\
\hline
\end{tabular}

\begin{tabular}{|c|c|c|c|c|c|c|}
\hline \multicolumn{2}{|c|}{ Reproduction at } & \multicolumn{2}{|c|}{$25 \%$} & \multicolumn{3}{c|}{ Concentration } \\
\hline Rep. & Day 3/4 & Day 5 & Day 6 & Day 7 & Day 8 & Total \\
\hline 1 & 4 & 0 & 12 & 0 & 14 & 30 \\
\hline 2 & 4 & 0 & 10 & 0 & 7 & 21 \\
\hline 3 & 0 & 5 & 8 & 0 & 13 & 26 \\
\hline 4 & 4 & 0 & 12 & 0 & 15 & 31 \\
\hline 5 & 3 & 0 & 10 & 0 & 12 & 25 \\
\hline 6 & 3 & 0 & 12 & 0 & 15 & 30 \\
\hline 7 & 3 & 0 & 13 & 0 & 12 & 28 \\
\hline 8 & 5 & 0 & 8 & 0 & 14 & 27 \\
\hline 9 & 0 & 5 & 11 & 0 & 15 & 31 \\
\hline 10 & 5 & 6 & 9 & 0 & 12 & 32 \\
\hline
\end{tabular}

\begin{tabular}{|c|c|c|c|c|c|c}
\hline \multicolumn{2}{|c|}{ Reproduction at } & \multicolumn{2}{c}{$100 \%$} & \multicolumn{2}{c}{ Concentration } \\
\hline Rep. & Day 3/4 & Day 5 & Diy 6 & Day 7 & Day 8 & Totel \\
\hline 1 & 7 & 0 & 0 & 0 & 12 & 19 \\
\hline 2 & 0 & 5 & 12 & 0 & 20 & 37 \\
\hline 3 & 0 & 3 & 9 & 0 & 12 & 24 \\
\hline 4 & 0 & 4 & 11 & 0 & 14 & 29 \\
\hline 5 & 4 & 0 & 2 & $\times$ & $X$ & 6 \\
\hline 6 & 3 & 0 & 10 & 0 & 10 & 23 \\
\hline 7 & 4 & 0 & 12 & 0 & 15 & 31 \\
\hline 8 & 5 & 0 & 11 & 0 & 17 & 33 \\
\hline 9 & 0 & 4 & 5 & 0 & 9 & 18 \\
\hline 10 & 0 & 5 & 8 & 0 & 15 & 28 \\
\hline
\end{tabular}

\begin{tabular}{|c|c|c|c|c|c|c|}
\hline \multicolumn{2}{|c|}{ Reproduction at } & \multicolumn{3}{c|}{$12.5 \%$} & \multicolumn{2}{c|}{ Concentration } \\
\hline Rep. & Day 3/4 & Day 5 & Day 6 & Day 7 & Day 8 & Total \\
\hline 1 & 3 & 9 & 0 & 16 & 0 & 28 \\
\hline 2 & 4 & 0 & 8 & 0 & 10 & 22 \\
\hline 3 & 0 & 5 & 9 & 0 & 12 & 26 \\
\hline 4 & 0 & 0 & 10 & 0 & 12 & 22 \\
\hline 5 & 4 & 5 & 10 & 0 & 16 & 35 \\
\hline 6 & 4 & 0 & 10 & 0 & 13 & 27 \\
\hline 7 & 5 & 0 & 12 & 0 & 16 & 33 \\
\hline 8 & 5 & 0 & 12 & 0 & 11 & 28 \\
\hline 9 & 0 & 1 & 0 & 0 & 10 & 20 \\
\hline 10 & 2 & 0 & 12 & 0 & 12 & 26 \\
\hline
\end{tabular}

\begin{tabular}{|c|c|c|c|c|c|c|}
\hline \multicolumn{2}{|c|}{ Reproduction at } & \multicolumn{9}{c|}{$50 \%$} \\
\hline Rep. & Day 3/4 & Day 5 & Day 6 & Day 7 & Day 8 & Total \\
\hline 1 & 5 & 9 & 0 & 0 & 14 & 28 \\
\hline 2 & 5 & 0 & 10 & 0 & 12 & 27 \\
\hline 3 & 0 & 5 & 12 & 0 & 13 & 30 \\
\hline 4 & 7 & 1 & 13 & 0 & 14 & 35 \\
\hline 5 & 2 & 0 & 8 & 0 & 8 & 18 \\
\hline 6 & 4 & 0 & 12 & 0 & 18 & 34 \\
\hline 7 & 4 & 0 & 14 & 0 & 14 & 32 \\
\hline 8 & 8 & 0 & 12 & 0 & 15 & 35 \\
\hline 9 & 0 & 3 & 4 & 0 & 5 & 12 \\
\hline 10 & 3 & 0 & 8 & 0 & 16 & 27 \\
\hline
\end{tabular}

\begin{tabular}{|c|c|c|c|c|c|c|}
\hline \multicolumn{2}{|c|}{ Reproduction at } & \multicolumn{7}{c|}{ MHSF } & Control \\
\hline Rep. & Day 3/4 & Day 5 & Day 6 & Day 7 & Day 8 & Total \\
\hline 1 & 6 & 0 & 4 & 0 & 12 & 22 \\
\hline 2 & 5 & 0 & 3 & 7 & 0 & 15 \\
\hline 3 & 6 & 0 & 6 & 14 & 0 & 26 \\
\hline 4 & 4 & 0 & 9 & 14 & 0 & 27 \\
\hline 5 & 7 & 0 & 10 & 13 & 0 & 30 \\
\hline 6 & 6 & 0 & 11 & 15 & 0 & 32 \\
\hline 7 & 0 & 6 & 10 & 0 & 0 & 16 \\
\hline 8 & 0 & 4 & 12 & 0 & 11 & 27 \\
\hline 9 & 0 & 8 & 12 & 0 & 12 & 32 \\
\hline 10 & 0 & 5 & 8 & 0 & 13 & 26 \\
\hline
\end{tabular}




\section{CHRONIC DEFINITIVE SURVIVAL AND REPRODUCTION TEST \\ Statistical Analyses}

Client:

WSRC

Sample Identification:

Test Date:

A-11

April 9, 2002

\section{Test for Normality}

Test Used: Kolmogorov D

$\begin{array}{ll}D= & N A \\ \text { critical } & \text { NA }\end{array}$

NA

\section{Test for Homogeneity of Variance}

Test Used: Bartlett's Test

$B=N A$

critical NA

\begin{tabular}{|c|c|c|c|c|c|c|}
\hline \multicolumn{7}{|c|}{ Test for Differences in Reproduction } \\
\hline \multicolumn{2}{|c|}{ Test Type Used: } & \multicolumn{5}{|c|}{$\begin{array}{r}\text { Non-Linear Regression - Logistic Model } \\
\text { Calculated Using JMP Software } \\
\% \text { Effluent }\end{array}$} \\
\hline Enect & MHSF & $6.2 \%$ & $12.5 \%$ & $25.0 \%$ & $50.0 \%$ & $100 \%$ \\
\hline Survival & $100 \%$ & $100 \%$ & $100 \%$ & $100 \%$ & $100 \%$ & $90 \%$ \\
\hline Prodicted & $100 \%$ & $100 \%$ & $100 \%$ & $100 \%$ & $100 \%$ & $100 \%$ \\
\hline Reproduction & 25.3 & 26.1 & 26.7 & 28.1 & 27.8 & 24.8 \\
\hline Prodictod & 26.5 & 26.5 & 26.5 & 26.5 & 26.5 & 26.5 \\
\hline Variance & 35.79 & 20.32 & 22.46 & 11.66 & 56.44 & 79.96 \\
\hline \multicolumn{4}{|c|}{ Text Parameters } & & MSD- & 6.35 \\
\hline & alpha & beta & Eamana & \multicolumn{2}{|c|}{$\begin{array}{l}\text { \% Reduction } \\
\text { CTC }=98.1 \%\end{array}$} & Pasa/Fail \\
\hline Survival & 3.00 & -200.00 & 0.00 & \multicolumn{2}{|c|}{$0.0 \%$} & Pass \\
\hline Reproduction & 4.78 & -200.00 & 26.47 & \multicolumn{2}{|c|}{$0.0 \%$} & Pass \\
\hline
\end{tabular}

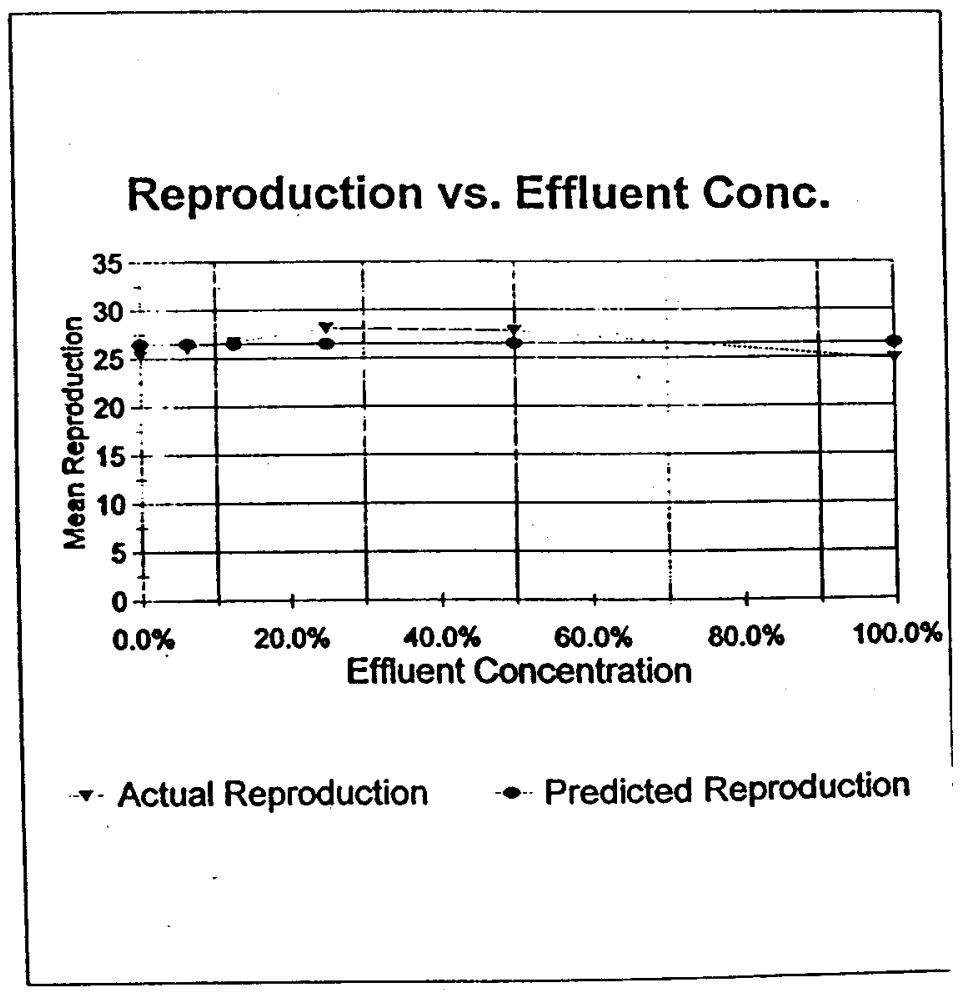

Summary

The reductions in survival and reproduction at the CTC are $<25 \%$ - Pass 


\title{
7 Day Chronic Definitive Survival and Reproduction Bioassay \\ Method: EPA/600/4-91/002
}

Test Organism:

Daphnia ambigua

Facility: WSRC

Sample ID: G-10

\author{
Aiken County \\ ETT\#: 19674
}

Sample ID: April 16, 2002 


\section{CHRONIC DEFINITIVE SURVIVAL AND REPRODUCTION TEST}

Survival and Reproduction Results

Client:

Sample Identification:

Test Date:

WSRC

G-10

April 16, 2002
County:

NPDESH:

ETT \#:
Aiken .

SC

19674

\begin{tabular}{|c|c|c|c|c|c|c|}
\hline Reproduction at & \multicolumn{5}{c|}{ MHSF } & Control \\
\hline Rep. & Day 3/4 & Day 5 & Day 6 & Day 7 & Day 8 & Total \\
\hline 1 & 5 & $X$ & $X$ & $X$ & $X$ & 5 \\
\hline 2 & 5 & 0 & 12 & 13 & 0 & 30 \\
\hline 3 & 5 & 0 & 10 & 14 & 0 & 29 \\
\hline 4 & 5 & 0 & 13 & 13 & 0 & 31 \\
\hline 5 & 5 & 10 & 0 & 15 & 0 & 30 \\
\hline 6 & 4 & 0 & 8 & 8 & 0 & 20 \\
\hline 7 & 5 & 0 & 11 & 13 & 0 & 29 \\
\hline 8 & 5 & 0 & 11 & 12 & 0 & 28 \\
\hline 9 & 5 & 7 & 0 & 11 & 0 & 23 \\
\hline 10 & 6 & 0 & 13 & $X$ & $X$ & 19 \\
\hline
\end{tabular}

\begin{tabular}{|c|c|c|c|c|c|c|}
\hline \multicolumn{2}{|c|}{ Reproduction at } & \multicolumn{6}{|c|}{$6.25 \%$} & \multicolumn{3}{c|}{ Concentration } \\
\hline Rep. & Day 3/4 & Day 5 & Day 6 & Day 7 & Day 8 & Total \\
\hline 1 & 4 & 0 & 11 & 13 & 0 & 28 \\
\hline 2 & 4 & 0 & 12 & 15 & 0 & 31 \\
\hline 3 & 5 & 0 & 10 & 10 & 0 & 25 \\
\hline 4 & 4 & 0 & 9 & $X$ & $X$ & 13 \\
\hline 5 & 4 & 0 & 12 & 14 & 0 & 30 \\
\hline 6 & 6 & 0 & 10 & 11 & 0 & 27 \\
\hline 7 & 4 & 0 & 13 & 12 & 0 & 29 \\
\hline 8 & 3 & 2 & 11 & $X$ & $X$ & 16 \\
\hline 9 & 5 & 0 & 8 & 12 & 0 & 25 \\
\hline 10 & 4 & 0 & 8 & 12 & 0 & 24 \\
\hline
\end{tabular}

\begin{tabular}{|c|c|c|c|c|c|c|}
\hline \multicolumn{2}{|c|}{ Reproduction at } & \multicolumn{3}{|c|}{$12.5 \%$} \\
\hline Rep. & Day 3/4 & Day 5 & Day 6 & Day 7 & Day 8 & Toal \\
\hline 1 & 5 & 0 & 11 & 15 & 0 & 31 \\
\hline 2 & 5 & 0 & 13 & 9 & 0 & 27 \\
\hline 3 & 5 & 0 & 12 & 10 & 0 & 27 \\
\hline 4 & 4 & 0 & 13 & 15 & 0 & 32 \\
\hline 5 & 5 & 0 & 12 & 11 & 0 & 28 \\
\hline 6 & 4 & 0 & 12 & 12 & 0 & 28 \\
\hline 7 & 4 & 0 & 11 & 8 & 0 & 23 \\
\hline 8 & 4 & 0 & 10 & 11 & 0 & 25 \\
\hline 9 & 5 & 8 & 0 & 14 & 0 & 27 \\
\hline 10 & 4 & 0 & 8 & 12 & 0 & 24 \\
\hline
\end{tabular}

\begin{tabular}{|c|c|c|c|c|c|c|}
\hline \multicolumn{3}{|c|}{ Reproduction at } & \multicolumn{3}{c|}{$25 \%$} & \multicolumn{2}{c|}{ Concentration } \\
\hline Rep. & Day 3/4 & Day 5 & Day 6 & Day 7 & Day 8 & Total \\
\hline 1 & 4 & 0 & 13 & $X$ & $X$ & 17 \\
\hline 2 & 4 & 0 & 8 & 7 & 0 & 19 \\
\hline 3 & 3 & 2 & 7 & 4 & 0 & 16 \\
\hline 4 & 5 & 0 & 12 & 14 & 0 & 31 \\
\hline 5 & 3 & 0 & 13 & 12 & 0 & 28 \\
\hline 6 & 5 & 0 & 12 & 14 & 0 & 31 \\
\hline 7 & 5 & 0 & 12 & $X$ & $X$ & 17 \\
\hline 8 & 5 & 0 & 13 & 13 & 0 & 31 \\
\hline 9 & 4 & $X$ & $X$ & $X$ & $X$ & 4 \\
\hline 10 & 5 & 0 & 13 & 11 & 0 & 29 \\
\hline
\end{tabular}

\begin{tabular}{|c|c|c|c|c|c|c|}
\hline Reproduction at & \multicolumn{6}{c|}{$50 \%$} \\
\hline Rep. & Day 3/4 & Day 5 & Day 6 & Day 7 & Day 8 & Toual \\
\hline 1 & 5 & 0 & 13 & 10 & 0 & 28 \\
\hline 2 & 6 & 0 & 10 & 12 & 0 & 28 \\
\hline 3 & 5 & 0 & 13 & $X$ & $X$ & 18 \\
\hline 4 & 4 & 0 & 12 & 15 & 0 & 31 \\
\hline 5 & 3 & 0 & 13 & 5 & 0 & 21 \\
\hline 6 & 3 & 0 & 10 & $X$ & $X$ & 13 \\
\hline 7 & 4 & $X$ & $X$ & $X$ & $X$ & 4 \\
\hline 8 & 4 & 0 & 12 & 11 & 0 & 27 \\
\hline 9 & 4 & 10 & 0 & 8 & 0 & 22 \\
\hline 10 & 6 & 0 & 11 & 10 & 0 & 27 \\
\hline
\end{tabular}

\begin{tabular}{|c|c|c|c|c|c|c}
\hline \multicolumn{2}{|c|}{ Reproduction at } & \multicolumn{4}{c}{$100 \%$} & \multicolumn{2}{c}{ Concentration } \\
\hline Rep. & Day 3/4 & Day 5 & Day6 & Day 7 & Day 8 & Total \\
\hline 1 & 5 & 0 & 14 & $X$ & $X$ & 19 \\
\hline 2 & 6 & 0 & 13 & $X$ & $X$ & 19 \\
\hline 3 & 0 & 0 & 11 & 11 & 0 & 22 \\
\hline 4 & 5 & 0 & 12 & 10 & 0 & 27 \\
\hline 5 & 4 & 7 & 0 & 14 & 0 & 25 \\
\hline 6 & 5 & 0 & 4 & 12 & 0 & 21 \\
\hline 7 & 4 & 0 & $X$ & $X$ & $X$ & 4 \\
\hline 8 & 5 & 0 & 13 & 11 & 0 & 29 \\
\hline 9 & 4 & 9 & 3 & $X$ & $X$ & 16 \\
\hline 10 & 4 & 8 & 0 & 11 & 0 & 23 \\
\hline
\end{tabular}




\section{CHRONIC DEFINITIVE SURVIVAL AND REPRODUCTION TEST \\ Statistical Analyses}

Client:

Sample Identification:

Test Date:
WSRC

G-10

April 16, 2002

\section{Test for Normality}

Test Used: Kolmogorov D

$\begin{array}{ll}D= & \text { NA } \\ \text { critical } & \text { NA }\end{array}$

NA
Test for Homogeneity of Variance

Test Used: Bartlett's Test

$B=N A$

critical NA

\begin{tabular}{|c|c|c|c|c|c|c|}
\hline \multicolumn{7}{|c|}{ Test for Differences in Reproduction } \\
\hline \multicolumn{2}{|l|}{ Test Type Usod: } & \multicolumn{5}{|c|}{$\begin{array}{r}\text { Non-Linear Regression - Logistic Model } \\
\text { Calculated Using MMP Sotiware } \\
\text { \% Eflluent }\end{array}$} \\
\hline Ereet & MHSF & $6.3 \%$ & $12.5 \%$ & $25.0 \%$ & $50.0 \%$ & $100 \%$ \\
\hline Servival & $90 \%$ & $100 \%$ & $100 \%$ & $90 \%$ & $80 \%$ & $20 \%$ \\
\hline Predictad & $100 \%$ & $100 \%$ & $100 \%$ & $100 \%$ & $100 \%$ & $100 \%$ \\
\hline Reproduction & 24.4 & 24.8 & 27.2 & 22.3 & 21.9 & 20.5 \\
\hline Prodicted & 25.3 & 24.9 & 24.5 & 23.8 & 22.5 & 20.1 \\
\hline Verience & 65.38 & 35.07 & 7.96 & 82.90 & 69.43 & 48.94 \\
\hline
\end{tabular}

\begin{tabular}{|c|c|c|c|c|c|c|}
\hline \multicolumn{4}{|c|}{ Text Parameters } & \multirow{2}{*}{\multicolumn{2}{|c|}{ \% Reduction C CTC }} & \multirow[b]{3}{*}{ Pass/Fail } \\
\hline & & & & & & \\
\hline & alpha & beta & Examma & $19.0 \%$ & $100.0 \%$ & \\
\hline Survival & 3.00 & -200.00 & 0.00 & $0.0 \%$ & $0.0 \%$ & Pass \\
\hline Reproduction & 2.57 & -2.38 & 25.27 & $4.4 \%$ & $20.4 \%$ & Pass \\
\hline
\end{tabular}

\section{Reproduction vs. Effluent Conc.}

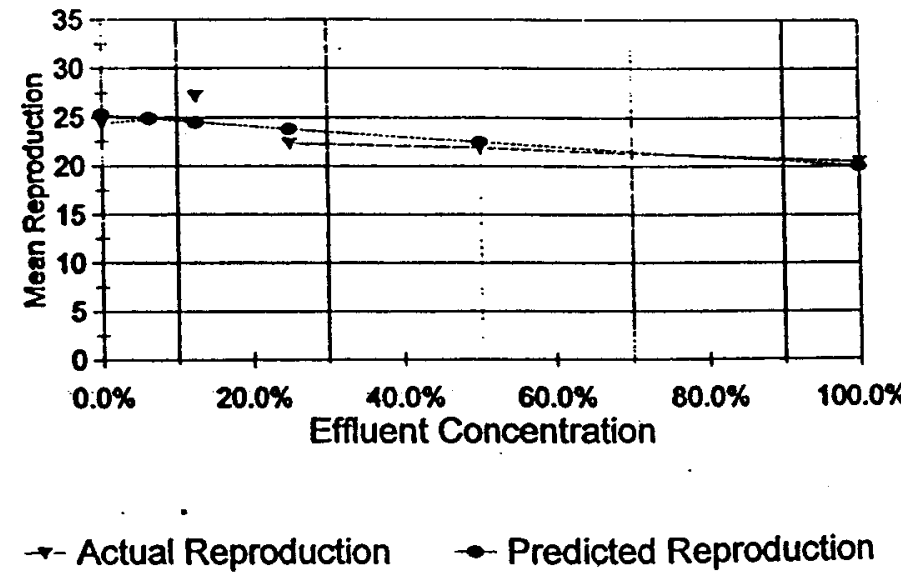

IC25 $=\quad>100 \%$

Summary

The reductions in survival and reproduction at the CTC are $<25 \%$ - Pass 


\section{CHRONIC DEFINITIVE SURVIVAL AND REPRODUCTION TEST \\ Water Chemistry}

Client: $\quad$ WSRC

Sample Identification: $\quad$ G-10

Test Date: April 16, 2002

pH (After Renewal)

\begin{tabular}{|l|r|r|r|r|r|r|}
\hline \multicolumn{7}{|c|}{ Test Concentration } \\
\hline & Control & $6.25 \%$ & $12.5 \%$ & $25.0 \%$ & $50.0 \%$ & $100 \%$ \\
\hline Initial & & 7.65 & 7.47 & 7.43 & 7.58 & 7.70 \\
\hline Day 1 & 7.19 & & 7.17 & 7.34 & 7.40 & 7.58 \\
\hline Day 2 & 7.31 & 7.23 & 7.24 & 7.43 & 7.61 & 7.79 \\
\hline Day 3 & 7.60 & 7.36 & 7.29 & 7.33 & 7.50 & 7.66 \\
\hline Day 4 & 7.42 & 7.25 & 7.31 & 7.33 & 7.59 & 7.75 \\
\hline Day 5 & 8.11 & 8.04 & 7.81 & 7.66 & 7.65 & 7.74 \\
\hline Day 6 & 7.72 & 7.50 & 7.37 & 7.36 & 7.50 & 7.76 \\
\hline
\end{tabular}

Dissolved Oxygen (After Renewal)

\begin{tabular}{|l|r|r|r|r|r|r|}
\hline & Control & $6.25 \%$ & $12.5 \%$ & $25.0 \%$ & $50.0 \%$ & $100 \%$ \\
\hline Lnitial & 8.1 & 8.1 & 8.2 & 8.2 & 8.2 & 8.2 \\
\hline Day 1 & 8.7 & 8.7 & 8.2 & 8.2 & 8.2 & 8.3 \\
\hline Day 2 & 8.4 & 8.4 & 8.3 & 8.3 & 8.2 & 8.2 \\
\hline Day 3 & 8.2 & 8.2 & 8.2 & 8.2 & 8.3 & 8.3 \\
\hline Day 4 & 8.2 & 8.2 & 8.2 & 8.3 & 8.3 & 8.3 \\
\hline Day 5 & 8.2 & 8.2 & 8.2 & 8.2 & 8.3 & 8.3 \\
\hline Day 6 & 8.1 & 8.1 & 8.1 & 8.1 & 8.2 & 8.3 \\
\hline
\end{tabular}

Test Results Reviewed and Approved By:
$\mathrm{pH}$ (Before Renewal)

\begin{tabular}{|l|r|r|r|r|r|r|}
\hline \multicolumn{7}{|c|}{ Test Concentration } \\
\hline & Control & $6.25 \%$ & $12.5 \%$ & $25.0 \%$ & $50.0 \%$ & $100 \%$ \\
\hline Day 1 & 7.37 & & 7.27 & 7.33 & 7.38 & 7.61 \\
\hline Day 2 & 7.25 & 7.32 & 7.46 & 7.72 & & \\
\hline Day 3 & 7.89 & 7.62 & 7.51 & 7.54 & 7.64 & 7.92 \\
\hline Day 4 & 7.36 & 7.43 & 7.51 & 7.61 & 7.65 & 7.79 \\
\hline Day 5 & 8.10 & 8.03 & 7.90 & 7.29 & 7.72 & 7.78 \\
\hline Day 6 & 7.95 & 7.77 & 7.66 & 7.52 & 7.66 & 7.87 \\
\hline Final & 6.99 & 7.13 & 7.21 & 7.51 & 7.65 & 8.07 \\
\hline
\end{tabular}

Dissolved Oxygen (Before Renewal)

\begin{tabular}{|l|r|r|r|r|r|r|}
\hline & Control & $6.25 \%$ & $12.5 \%$ & $25.0 \%$ & $50.0 \%$ & $100 \%$ \\
\hline Day 1 & 8.2 & 8.2 & 8.3 & 8.3 & 8.3 & 8.3 \\
\hline Day 2 & 8.1 & 8.0 & 8.0 & 8.0 & & \\
\hline Day 3 & 8.3 & 8.3 & 8.3 & 8.3 & 8.3 & 8.3 \\
\hline Day 4 & 8.2 & 8.2 & 8.2 & 8.3 & 8.3 & 8.3 \\
\hline Day 5 & 8.2 & 8.3 & 8.3 & 8.3 & 8.3 & 8.6 \\
\hline Day 6 & 8.3 & 8.4 & 8.4 & 8.6 & 8.6 & 8.7 \\
\hline Final & 8.3 & 8.2 & 8.2 & 8.2 & 8.2 & 8.2 \\
\hline
\end{tabular}

Temperature

\begin{tabular}{|l|r|}
\hline \multicolumn{2}{|c|}{ Incubator ${ }^{\circ} \mathrm{C}$} \\
\hline Initial & 24.4 \\
\hline Day 1 & 24.7 \\
\hline Day 2 & 24.8 \\
\hline Day 3 & 24.5 \\
\hline Day 4 & 24.7 \\
\hline Day 5 & 24.7 \\
\hline Day 6 & 24.7 \\
\hline Final & 24.7 \\
\hline
\end{tabular}




\section{Day Chronic Definitive Survival and Reproduction Bioassay Method: EPA/600/4-91/002}

Test Organism:

Daphnia ambigua

Facility: WSRC

Sample ID: A-01

Aiken County

ETT\#: 19800

Sample ID: May 7, 2002 


\section{CHRONIC DEFINITIVE SURVIVAL AND REPRODUCTION TEST}

Survival and Reproduction Results

Client: WSRC

Sample Identification: A-01

Test Date: May 7, 2002

\begin{tabular}{|c|c|c|c|c|c|c|}
\hline \multicolumn{2}{|c|}{ Reproduction at } & MHSF Control \\
\hline Rep. & Day 3/4 & Day 5 & Day 6 & Day 7 & Day 8 & Toual \\
\hline 1 & 4 & 0 & 8 & 13 & 0 & 25 \\
\hline 2 & 8 & 0 & 10 & 11 & 0 & 29 \\
\hline 3 & 5 & $X$ & $X$ & $X$ & $X$ & 5 \\
\hline 4 & 4 & 11 & 11 & 0 & 0 & 26 \\
\hline 5 & 4 & 0 & 10 & 12 & 0 & 26 \\
\hline 6 & 3 & 10 & 11 & 0 & 0 & 24 \\
\hline 7 & 5 & 0 & 12 & 16 & 0 & 33 \\
\hline 8 & 4 & 9 & 10 & 0 & 0 & 23 \\
\hline 9 & 5 & 0 & 11 & 16 & 0 & 32 \\
\hline 10 & 5 & 0 & 10 & 17 & 0 & 32 \\
\hline
\end{tabular}

\begin{tabular}{|c|c|c|c|c|c|c|}
\hline \multicolumn{2}{|c|}{ Reproduction at } & \multicolumn{3}{c|}{$12.5 \%$} & \multicolumn{3}{c|}{ Concentration } \\
\hline Rep. & Day 3/4 & Day 5 & Day 6 & Day 7 & Day 8 & Total \\
\hline 1 & 5 & 0 & 11 & 15 & 0 & 31 \\
\hline 2 & 4 & 11 & 14 & 0 & 0 & 29 \\
\hline 3 & 4 & 0 & 12 & 15 & 0 & 31 \\
\hline 1 & 3 & 9 & 15 & 0 & 0 & 27 \\
\hline 5 & 4 & 0 & 11 & 15 & 0 & 30 \\
\hline 6 & 4 & 10 & 11 & 0 & 0 & 25 \\
\hline 7 & 6 & 0 & 11 & 15 & 0 & 32 \\
\hline 8 & 6 & 0 & 12 & 14 & 0 & 32 \\
\hline 9 & 5 & 0 & 10 & 14 & 0 & 29 \\
\hline 10 & 5 & 0 & 11 & 17 & 0 & 33 \\
\hline
\end{tabular}

\begin{tabular}{|c|c|c|c|c|c|c|}
\hline Reproduction at & \multicolumn{6}{|c|}{$50 \%$} \\
\hline Rep. & Day 3/4 & Day 5 & Day 6 & Day 7 & Day 8 & Toal \\
\hline 1 & 4 & 0 & 7 & 18 & 0 & 29 \\
\hline 2 & 3 & 0 & 0 & 2 & 0 & 5 \\
\hline 3 & 3 & 0 & 8 & 14 & 0 & 25 \\
\hline 4 & 4 & 9 & 12 & 0 & 0 & 25 \\
\hline 5 & 6 & 0 & 13 & 18 & 0 & 37 \\
\hline 6 & 4 & 11 & 13 & 0 & 0 & 28 \\
\hline 7 & 5 & 0 & 2 & 16 & 0 & 23 \\
\hline 8 & 5 & 0 & 10 & 17 & 0 & 32 \\
\hline 9 & 6 & 0 & 15 & 17 & 0 & 38 \\
\hline 10 & 5 & 0 & 8 & 17 & 0 & 30 \\
\hline
\end{tabular}

County:

NPDESH: $\quad$ SC

ETT \#:

\begin{tabular}{|c|c|c|c|c|c|c|}
\hline \multicolumn{2}{|c|}{ Reproduction at } & \multicolumn{6}{c|}{$6.25 \%$} \\
\hline Rep. & Day 3/4 & Day 5 & Day 6 & Day 7 & Day 8 & Total \\
\hline 1 & 6 & 0 & 12 & 16 & 0 & 34 \\
\hline 2 & 9 & 11 & 14 & 0 & 0 & 34 \\
\hline 3 & 6 & 10 & 0 & 17 & 0 & 33 \\
\hline 4 & 4 & 6 & 13 & 0 & 0 & 23 \\
\hline 5 & 5 & 0 & 11 & 16 & 0 & 32 \\
\hline 6 & 9 & 0 & 4 & 9 & 0 & 22 \\
\hline 7 & 4 & 0 & 10 & 16 & 0 & 30 \\
\hline 8 & 0 & 0 & 5 & 10 & 0 & 15 \\
\hline 9 & 6 & 0 & 9 & 13 & 0 & 28 \\
\hline 10 & 6 & 0 & 11 & 14 & 0 & 31 \\
\hline
\end{tabular}

\begin{tabular}{|c|c|c|c|c|c|c|}
\hline \multicolumn{3}{|c|}{ Reproduction at } & \multicolumn{4}{c|}{$25 \%$} \\
\hline Rep. & Day 3/4 & Day 5 & Day 6 & Day 7 & Day 8 & Toala \\
\hline 1 & 5 & 0 & 9 & 12 & 0 & 26 \\
\hline 2 & 4 & 8 & 7 & 0 & 0 & 19 \\
\hline 3 & 6 & 0 & 12 & 16 & 0 & 34 \\
\hline 4 & 4 & 12 & 14 & 0 & 0 & 30 \\
\hline 5 & 5 & 0 & 14 & 17 & 0 & 36 \\
\hline 6 & 5 & 9 & 13 & 0 & 0 & 27 \\
\hline 7 & 4 & 0 & 14 & 7 & 0 & 25 \\
\hline 8 & 4 & 0 & 3 & 17 & 0 & 24 \\
\hline 9 & 4 & 0 & 14 & 19 & 0 & 37 \\
\hline 10 & 5 & 0 & 10 & 19 & 0 & 34 \\
\hline
\end{tabular}

\begin{tabular}{|c|c|c|c|c|c|c|}
\hline \multicolumn{2}{|c|}{ Reproduction at } & \multicolumn{2}{c|}{$100 \%$} & \multicolumn{2}{c|}{ Concentration } \\
\hline Rep. & Dey 3/4 & Day 5 & Day 6 & Day 7 & Day 8 & Tocal \\
\hline 1 & 5 & 0 & 10 & 15 & 0 & 30 \\
\hline 2 & 3 & $X$ & $X$ & $X$ & $X$ & 3 \\
\hline 3 & 5 & 0 & 13 & 16 & 0 & 34 \\
\hline 4 & 4 & 12 & 15 & 0 & 0 & 31 \\
\hline 5 & 4 & 9 & 0 & 16 & 0 & 29 \\
\hline 6 & 4 & 8 & 14 & 0 & 0 & 26 \\
\hline 7 & 5 & 0 & 9 & 12 & 0 & 26 \\
\hline 8 & 5 & 12 & 0 & 18 & 0 & 35 \\
\hline 9 & 5 & 10 & 0 & 17 & 0 & 32 \\
\hline 10 & 5 & 0 & 10 & 18 & 0 & 33 \\
\hline
\end{tabular}




\section{CHRONIC DEFINITIVE SURVIVAL AND REPRODUCTION TEST \\ Statistical Analyses}

Client:

Sample Identification:

Test Date:
WSRC

A-01

May 7, 2002

\section{Test for Normality}

Test Used: Kolmogorov D

$$
D=\quad N A
$$$$
\text { critical NA }
$$

NA

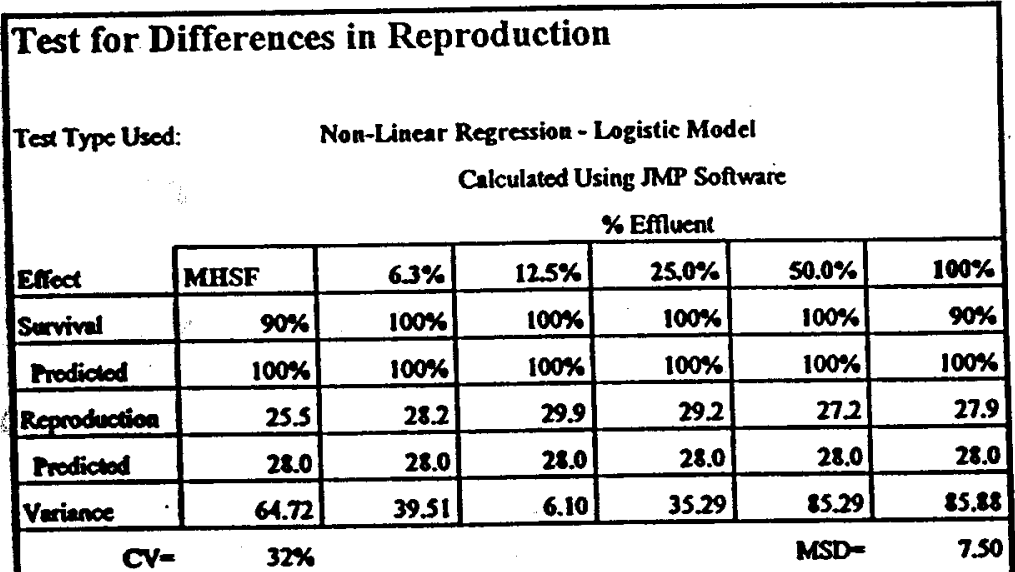

Tex Parameders

\begin{tabular}{|c|c|c|c|c|c|}
\hline \multicolumn{4}{|c|}{ Iex raluber } & \multirow{2}{*}{$\begin{array}{l}\text { \% Reduction } \\
\text { CTC }=100 \%\end{array}$} & \multirow[b]{2}{*}{ Peserfall } \\
\hline & alpha & beta & ganama & & \\
\hline Survival & 3.00 & -200.00 & 0.00 & $0.0 \%$ & Pass \\
\hline Reproduction & 4.78 & -200.00 & 28.00 & $0.0 \%$ & Pass \\
\hline & $1 C 25=$ & $>100 \%$ & & & \\
\hline
\end{tabular}

Test for Homogeneity of Variance

Test Used: Bartlett's Test

$B=N A$

critical NA

NA

\section{Reproduction vs. Effluent Conc.}

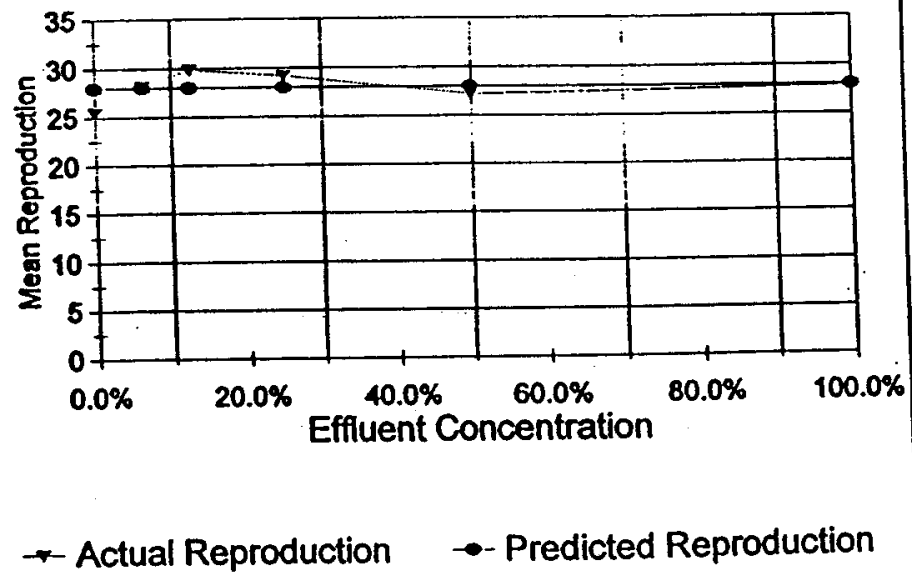

\section{Summary}

The reductions in survival and reproduction at the CTC are $<25 \%$ - Pass 


\section{CHRONIC DEFINITIVE SURVIVAL AND REPRODUCTION TEST \\ Water Chemistry}

Client:

Sample Identification:

WSRC

Test Date:

May 7, 2002

pH (After Renewal)

\begin{tabular}{|l|r|r|r|r|r|r|}
\hline \multicolumn{7}{|c|}{ Test Concentration } \\
\hline & Control & $6.25 \%$ & $12.5 \%$ & $25.0 \%$ & $50.0 \%$ & $100 \%$ \\
\hline Initial & 7.22 & 7.25 & 7.21 & 7.13 & 7.09 & 7.09 \\
\hline Day 1 & 7.24 & 7.27 & 7.10 & 7.98 & 7.01 & 6.98 \\
\hline Day 2 & 7.96 & 7.17 & 7.12 & 7.10 & 7.06 & 7.12 \\
\hline Day 3 & 7.48 & 7.96 & 6.86 & 6.86 & 6.76 & 6.69 \\
\hline Day 4 & 7.99 & 7.70 & 7.45 & 7.37 & 7.37 & 7.38 \\
\hline Day 5 & 7.43 & 7.40 & 7.22 & 7.20 & 7.25 & \\
\hline Day 6 & 6.92 & 6.90 & 6.84 & 6.92 & 6.92 & 7.16 \\
\hline
\end{tabular}

Dissolved Oxygen (After Renewal)

\begin{tabular}{|l|r|r|r|r|r|r|}
\hline & Control & $6.25 \%$ & $12.5 \%$ & $25.0 \%$ & $50.0 \%$ & $100 \%$ \\
\hline Initial & 8.2 & 8.1 & 8.1 & 8.1 & 8.2 & 8.1 \\
\hline Day 1 & 8.2 & 8.4 & 8.6 & 8.6 & 8.7 & 8.7 \\
\hline Day 2 & 8.3 & 8.2 & 8.3 & 8.3 & 8.4 & 8.4 \\
\hline Day 3 & 8.2 & 8.2 & 8.3 & 8.3 & 8.3 & 8.3 \\
\hline Day 4 & 8.3 & 8.3 & 8.4 & 8.4 & 8.4 & 8.4 \\
\hline Day 5 & 8.4 & 8.4 & 8.4 & 8.2 & 8.3 & 8.4 \\
\hline Day 6 & 8.5 & 8.8 & 8.9 & 8.9 & 8.9 & 8.9 \\
\hline
\end{tabular}

$\mathrm{pH}$ (Before Renewal)

\begin{tabular}{|l|r|r|r|r|r|r|}
\hline & Control & $6.25 \%$ & $12.5 \%$ & $25.0 \%$ & $50.0 \%$ & $100 \%$ \\
\hline Day 1 & 7.29 & 7.32 & & 6.96 & 7.03 & 7.16 \\
\hline Day 2 & 7.85 & 7.19 & 7.14 & 7.12 & 7.12 & 7.24 \\
\hline Day 3 & 7.72 & 8.21 & 7.17 & 7.16 & 7.19 & 7.23 \\
\hline Day 4 & 8.10 & 7.92 & 7.60 & 7.58 & 7.52 & 7.51 \\
\hline Day 5 & 7.74 & 7.65 & 7.63 & 7.47 & 7.48 & \\
\hline Day 6 & 7.11 & 7.38 & 7.40 & 7.21 & 7.16 & 7.15 \\
\hline Final & 7.61 & 7.85 & 7.22 & 7.21 & & \\
\hline
\end{tabular}

Dissolved Oxygen (Before Renewal)

\begin{tabular}{|l|r|r|r|r|r|r|}
\hline & Control & $6.25 \%$ & $12.5 \%$ & $25.0 \%$ & $50.0 \%$ & $100 \%$ \\
\hline Day 1 & 7.5 & 7.0 & & 7.7 & 7.6 & 7.8 \\
\hline Day 2 & 8.1 & 8.2 & 8.2 & 8.2 & 8.2 & 8.7 \\
\hline Day 3 & 8.2 & 8.2 & 8.2 & 8.2 & 8.3 & 8.5 \\
\hline Day 4 & 8.3 & 8.4 & 8.4 & 8.4 & 8.5 & \\
\hline Day 5 & 8.2 & 8.2 & 8.3 & 8.3 & 8.3 & \\
\hline Day 6 & 8.1 & 8.2 & 8.2 & 8.2 & 8.2 & 8.0 \\
\hline Final & 8.2 & 8.2 & 8.1 & 8.2 & & \\
\hline
\end{tabular}

Test Results Reviewed and Approved By:

Temperature
\begin{tabular}{|l|r|}
\hline \multicolumn{1}{|c|}{ Incubator ${ }^{\circ} \mathrm{C}$} \\
\hline Initial & 24.8 \\
\hline Day 1 & 24.7 \\
\hline Day 2 & 24.8 \\
\hline Day 3 & 25.0 \\
\hline Day 4 & 24.9 \\
\hline Day 5 & 24.8 \\
\hline Day 6 & 24.8 \\
\hline Final & 24.8 \\
\hline
\end{tabular}




\section{Day Chronic Definitive Survival and Reproduction Bioassay \\ Method: EPA/600/4-91/002}

Test Organism:

Daphnia ambigua

Facility: WSRC

Sample ID: A-11

\section{Aiken County}

ETT\#: 19801

Sample ID: May 7, 2002 


\section{CHRONIC DEFINITIVE SURVIVAL AND REPRODUCTION TEST Survival and Reproduction Results}

Client:
Sample Identification:
Test Date:
\begin{tabular}{|c|c|c|c|c|c|c|}
\hline Reproduction at \\
\hline Rep. & Day 3/4 & Day 5 & Day 6 & Day 7 & Day 8 & Total \\
\hline 1 & 4 & 0 & 8 & 13 & 0 & 25 \\
\hline 2 & 8 & 0 & 10 & 11 & 0 & 29 \\
\hline 3 & 5 & $X$ & $X$ & $X$ & $X$ & 5 \\
\hline 4 & 4 & 11 & 11 & 0 & 0 & 26 \\
\hline 5 & 4 & 0 & 10 & 12 & 0 & 26 \\
\hline 6 & 3 & 10 & 11 & 0 & 0 & 24 \\
\hline 7 & 5 & 0 & 12 & 16 & 0 & 33 \\
\hline 8 & 4 & 9 & 10 & 0 & 0 & 23 \\
\hline 9 & 5 & 0 & 11 & 16 & 0 & 32 \\
\hline 10 & 5 & 0 & 10 & 17 & 0 & 32 \\
\hline
\end{tabular}

$\begin{array}{ll}\text { County: } & \text { Aiken } \\ \text { NPDESH: } & \text { SC }\end{array}$

ETT \#: $\quad 19801$

\begin{tabular}{|c|c|c|c|c|c|c|}
\hline Reproduction at & \multicolumn{4}{c|}{$12.5 \%$} & \multicolumn{3}{c|}{ Concentration } \\
\hline Rep. & Day 3/4 & Day 5 & Day 6 & Day 7 & Day 8 & Total \\
\hline 1 & 4 & 0 & 12 & 11 & 0 & 27 \\
\hline 2 & 4 & 10 & 0 & 5 & 0 & 19 \\
\hline 3 & 5 & 0 & 12 & 15 & 0 & 32 \\
\hline 4 & 0 & 8 & 0 & 10 & 0 & 18 \\
\hline 5 & 9 & 0 & 12 & 11 & 0 & 32 \\
\hline 6 & 4 & 10 & 15 & 0 & 0 & 29 \\
\hline 7 & 3 & $X$ & $X$ & $X$ & $X$ & 3 \\
\hline 8 & 1 & 0 & 7 & 12 & 0 & 20 \\
\hline 9 & 5 & 0 & 13 & 16 & 0 & 34 \\
\hline 10 & 4 & 0 & 6 & 14 & 0 & 24 \\
\hline
\end{tabular}

\begin{tabular}{|c|c|c|c|c|c|c|}
\hline \multicolumn{2}{|c|}{ Reproduction at } & \multicolumn{2}{c|}{$6.25 \%$} & \multicolumn{3}{c|}{ Concentration } \\
\hline Rep. & Day 3/4 & Day 5 & Day 6 & Day 7 & Day 8 & Toual \\
\hline 1 & 4 & 0 & 2 & $X$ & $X$ & 6 \\
\hline 2 & 5 & 0 & 13 & 16 & 0 & 34 \\
\hline 3 & 6 & 9 & 10 & 0 & 0 & 25 \\
\hline 4 & 4 & 8 & 10 & 0 & 0 & 22 \\
\hline 5 & 5 & 0 & 11 & 14 & 0 & 30 \\
\hline 6 & 3 & 10 & 14 & 0 & 0 & 27 \\
\hline 7 & 5 & 7 & 15 & 0 & 0 & 27 \\
\hline 8 & 4 & 11 & 0 & 13 & 0 & 28 \\
\hline 9 & 5 & 0 & 13 & 13 & 0 & 31 \\
\hline 10 & 4 & 0 & 8 & 10 & 0 & 22 \\
\hline
\end{tabular}

\begin{tabular}{|c|c|c|c|c|c|c|}
\hline \multicolumn{2}{|c|}{ Reproduction at } & \multicolumn{3}{c|}{ Concentration } \\
\hline Rep. & Day 3/4 & Day S & Day 6 & Day 7 & Day & Total \\
\hline 1 & 4 & 0 & 13 & 12 & 0 & 29 \\
\hline 2 & 2 & 9 & 15 & 0 & 0 & 26 \\
\hline 3 & 5 & 0 & 2 & 17 & 0 & 24 \\
\hline 4 & 3 & 11 & 12 & 0 & 0 & 26 \\
\hline 5 & 5 & 0 & 13 & 16 & 0 & 34 \\
\hline 6 & 4 & 12 & 14 & 0 & 0 & 30 \\
\hline 7 & 3 & 11 & 12 & 0 & 0 & 26 \\
\hline 8 & 4 & 0 & 13 & 15 & 0 & 32 \\
\hline 9 & male & $\mathrm{L}$ & $\mathrm{L}$ & $\mathrm{L}$ & $\mathrm{L}$ & $\mathrm{L}$ \\
\hline 10 & 5 & 0 & 12 & 12 & 0 & 29 \\
\hline
\end{tabular}

\begin{tabular}{|c|c|c|c|c|c|c|}
\hline \multicolumn{2}{|c|}{ Reproduction at } & \multicolumn{2}{c|}{$25 \%$} & \multicolumn{2}{c|}{ Concentration } \\
\hline Rep. & Day 3/4 & Day 5 & Day 6 & Day 7 & Day 8 & Total \\
\hline 1 & 5 & 0 & 14 & 13 & 0 & 32 \\
\hline 2 & 3 & 9 & 12 & 0 & 0 & 24 \\
\hline 3 & 5 & 0 & 12 & 12 & 0 & 29 \\
\hline 4 & 4 & 8 & 13 & 0 & 0 & 25 \\
\hline 5 & 5 & 0 & 10 & 10 & 0 & 25 \\
\hline 6 & 3 & 7 & 13 & 0 & 0 & 23 \\
\hline 7 & 3 & 10 & 13 & 0 & 0 & 26 \\
\hline 8 & 4 & 0 & 0 & 14 & 0 & 18 \\
\hline 9 & 5 & 0 & 11 & 16 & 0 & 32 \\
\hline 10 & 4 & 0 & 10 & 13 & 0 & 27 \\
\hline
\end{tabular}

\begin{tabular}{|c|c|c|c|c|c|c}
\hline \multicolumn{2}{|c|}{ Reproduction at } & \multicolumn{2}{c}{$100 \%$} & \multicolumn{2}{c}{ Concentration } \\
\hline Rep. & Day 3/4 & Day 5 & Day 6 & Day 7 & Day 8 & Total \\
\hline 1 & 5 & 0 & 2 & 0 & 0 & 7 \\
\hline 2 & 3 & 10 & 14 & 0 & 0 & 27 \\
\hline 3 & 2 & 0 & 6 & 13 & 0 & 21 \\
\hline 4 & 4 & 7 & 7 & 0 & 0 & 18 \\
\hline 5 & 4 & 0 & 5 & 17 & 0 & 26 \\
\hline 6 & 4 & 3 & 0 & 7 & 0 & 14 \\
\hline 7 & 4 & 5 & 7 & 0 & 0 & 16 \\
\hline 8 & 2 & 0 & 7 & 8 & 0 & 17 \\
\hline 9 & 5 & 0 & 13 & 14 & 0 & 32 \\
\hline 10 & 4 & 0 & 9 & 16 & 0 & 29 \\
\hline
\end{tabular}




\section{CHRONIC DEFINITIVE SURVIVAL AND REPRODUCTION TEST \\ Statistical Analyses}

Client:

Sample Identification:

WSRC

Test Date:

A-11

May 7, 2002

\begin{tabular}{|lll}
\hline Test for Normality & & \\
& & \\
Test Used: Kolmogorov D & D & NA \\
& critical & NA
\end{tabular}

NA

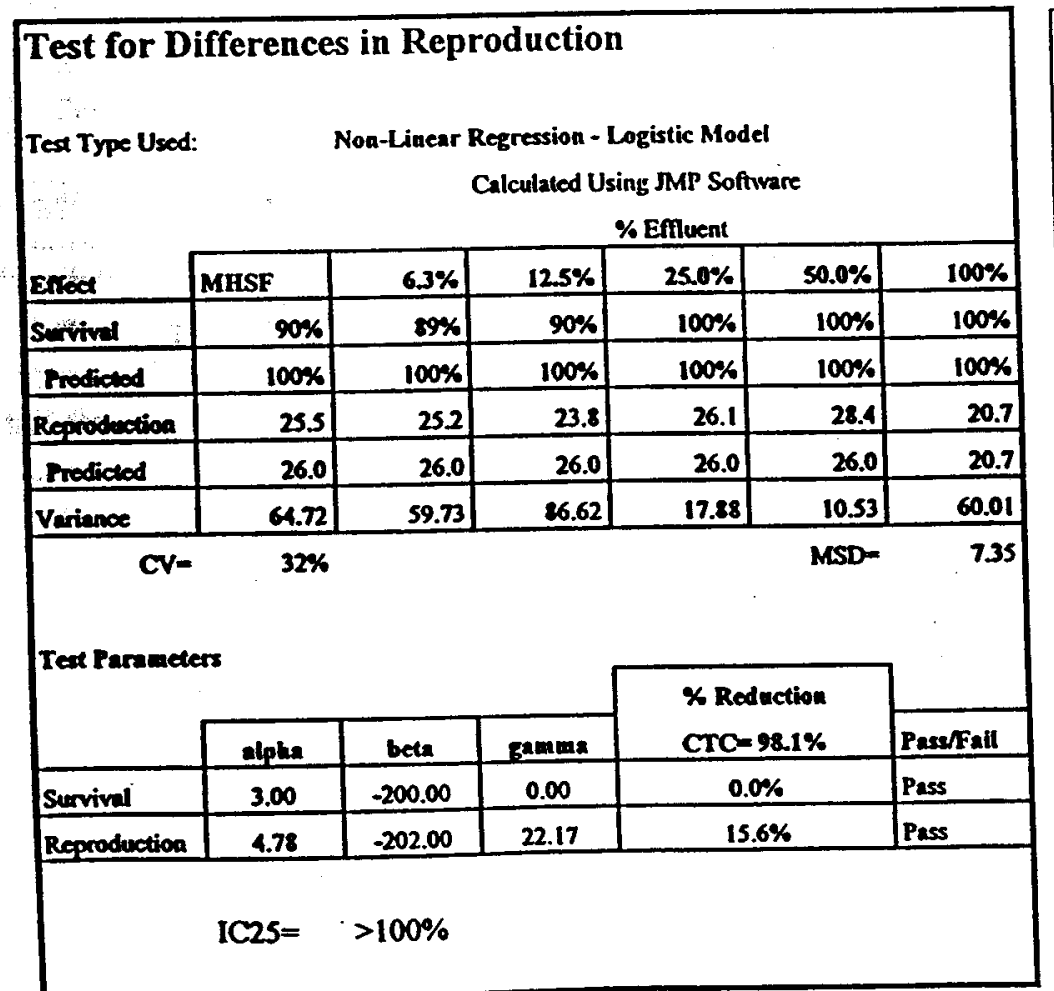

NA

Test for Homogeneity of Variance

Test Used: Bartlett's Test

$B=N A$

critical NA

Reproduction vs. Effluent Conc.

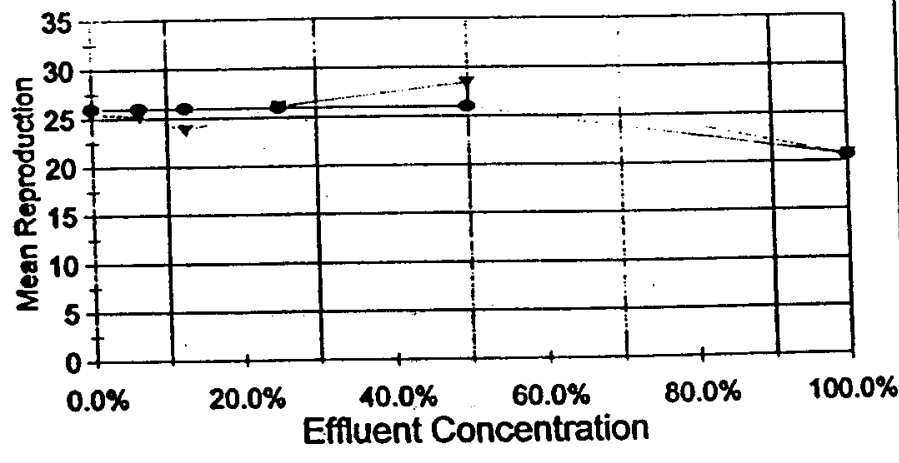

Actual Reproduction $\rightarrow$ Predicted Reproduction

Summary

The reductions in survival and reproduction at the CTC are $<25 \%$ - Pass 


\section{CHRONIC DEFINITIVE SURVIVAL AND REPRODUCTION TEST Water Chemistry}

Client:

Sample Identification:

Test Date:
WSRC

A-11

May 7, 2002
pH (After Renewal)

\begin{tabular}{|l|r|r|r|r|r|r|}
\hline \multicolumn{7}{|c|}{ Test Concentration } \\
\hline & Control & $6.25 \%$ & $12.5 \%$ & $25.0 \%$ & $50.0 \%$ & $100 \%$ \\
\hline Initial & 7.22 & 7.84 & 7.64 & 7.53 & 7.49 & 7.38 \\
\hline Day 1 & 7.24 & 6.85 & 6.81 & 6.78 & 6.80 & 6.83 \\
\hline Day 2 & 7.96 & 6.52 & 6.86 & 6.80 & 6.81 & 6.86 \\
\hline Day 3 & 7.48 & 6.86 & 6.83 & 6.79 & 6.79 & 6.81 \\
\hline Day 4 & 7.99 & 6.71 & 6.75 & 6.76 & 6.74 & 6.79 \\
\hline Day 5 & 7.43 & 7.12 & 7.05 & 7.00 & 7.00 & 7.02 \\
\hline Day 6 & 6.92 & 7.14 & 7.02 & 6.99 & 6.98 & 7.00 \\
\hline
\end{tabular}

\section{Dissolved Oxygen (After Renewal)}

\begin{tabular}{|l|r|r|r|r|r|r|}
\hline & \multicolumn{7}{|c|}{ Test Concentration } \\
\hline Initial & 8.2 & 8.1 & 8.2 & 8.2 & 8.2 & 8.2 \\
\hline Day 1 & 8.2 & 8.2 & 8.7 & 8.7 & 8.7 & 8.9 \\
\hline Day 2 & 8.3 & 8.2 & 8.2 & 8.3 & 8.4 & 8.4 \\
\hline Day 3 & 8.2 & 8.3 & 8.3 & 8.3 & 8.3 & 8.5 \\
\hline Day 4 & 8.3 & 8.3 & 8.3 & 8.3 & 8.4 & 8.4 \\
\hline Day 5 & 8.4 & 8.4 & 8.4 & 8.5 & 8.5 & 8.4 \\
\hline Day 6 & 8.5 & 9.0 & 9.0 & 9.0 & 9.0 & 9.0 \\
\hline
\end{tabular}

$\mathrm{pH}$ (Before Renewal)

\begin{tabular}{|l|r|r|r|r|r|r|}
\hline \multicolumn{7}{|c|}{ Test Concentration } \\
\hline & Control & $6.25 \%$ & $12.5 \%$ & $25.0 \%$ & $50.0 \%$ & $100 \%$ \\
\hline Day 1 & 7.29 & 6.81 & 6.79 & 6.70 & 6.76 & 6.74 \\
\hline Day 2 & 7.85 & 6.58 & 6.68 & 6.73 & 6.71 & 6.76 \\
\hline Day 3 & 7.72 & 6.78 & 6.73 & 6.72 & 6.73 & 6.75 \\
\hline Day 4 & 8.10 & 6.63 & 6.71 & 6.74 & 6.72 & 6.73 \\
\hline Day 5 & 7.74 & 7.27 & 7.25 & 7.21 & 7.19 & 7.19 \\
\hline Day 6 & 7.11 & 7.05 & 7.02 & 7.00 & 6.95 & 6.99 \\
\hline Final & 7.61 & 8.03 & 7.28 & 6.99 & 6.98 & 7.07 \\
\hline
\end{tabular}

Dissolved Oxygen (Before Renewal)

\begin{tabular}{|l|r|r|r|r|r|r|}
\hline & Control & $6.25 \%$ & $12.5 \%$ & $25.0 \%$ & $50.0 \%$ & $100 \%$ \\
\hline Day 1 & 7.5 & 7.6 & 7.4 & 7.7 & 7.4 & 7.4 \\
\hline Day 2 & 8.1 & 8.4 & 8.4 & 8.3 & 8.3 & 8.3 \\
\hline Day 3 & 8.2 & 8.2 & 8.3 & 8.3 & 8.3 & 8.4 \\
\hline Day 4 & 8.3 & 8.3 & 8.3 & 8.4 & 8.4 & 8.5 \\
\hline Day 5 & 8.2 & 8.3 & 8.4 & 8.3 & 8.3 & 8.4 \\
\hline Day 6 & 8.1 & 8.1 & 8.1 & 8.1 & 8.1 & 8.1 \\
\hline Final & 8.2 & 8.2 & 8.1 & 8.1 & 8.2 & 8.2 \\
\hline
\end{tabular}

Test Results Reviewed and Approved By:

Temperature
\begin{tabular}{|l|r|}
\hline \multicolumn{1}{|c|}{ Incubator ${ }^{\circ} \mathrm{C}$} \\
\hline Initia & 24.8 \\
\hline Day 1 & 24.7 \\
\hline Day 2 & 24.8 \\
\hline Day 3 & 25.0 \\
\hline Day 4 & 24.9 \\
\hline Day 5 & 24.8 \\
\hline Day 6 & 24.8 \\
\hline Final & 24.8 \\
\hline
\end{tabular}




\section{Day Chronic Definitive Survival and Reproduction Bioassay \\ Method: EPA/600/4-91/002}

Test Organism: Daphnia ambigua

Facility: WSRC

Sample ID: G-10

Aiken County

ETT\#: 19850

Sample ID: May 14, 2002 


\section{CHRONIC DEFINITIVE SURVIVAL AND REPRODUCTION TEST \\ Survival and Reproduction Results}

Client: WSRC/G-10

Sample Identification:

Test Date:

May 14,2002

\begin{tabular}{|c|c|c|c|c|c|c|}
\hline Reproduction at & \multicolumn{7}{c|}{ MHSF } & Control \\
\hline Rep. & Day 3/4 & Day 5 & Day 6 & Day 7 & Day 8 & Total \\
\hline 1 & 3 & 6 & 0 & 11 & 0 & 20 \\
\hline 2 & 3 & 7 & 0 & 10 & 0 & 20 \\
\hline 3 & 3 & 8 & 0 & 15 & 0 & 26 \\
\hline 4 & 4 & 9 & 14 & 0 & 0 & 27 \\
\hline 5 & 3 & 9 & 0 & 13 & 0 & 25 \\
\hline 6 & 4 & 7 & 0 & 12 & 0 & 23 \\
\hline 7 & 4 & 11 & 0 & 14 & 0 & 29 \\
\hline 8 & 0 & 5 & 12 & 8 & 0 & 25 \\
\hline 9 & 4 & 8 & 0 & 14 & 0 & 26 \\
\hline 10 & 5 & 10 & 0 & 14 & 0 & 29 \\
\hline
\end{tabular}

\begin{tabular}{|c|c|c|c|c|c|c|}
\hline Reproduction at & \multicolumn{1}{|c|}{$12.5 \%$} & \multicolumn{1}{c|}{ Concentration } \\
\hline Rep. & Day 3/4 & Day 5 & Day 6 & Day 7 & Day 8 & Total \\
\hline 1 & 4 & 8 & 0 & 12 & 0 & 24 \\
\hline 2 & 0 & 10 & 10 & 1 & 0 & 21 \\
\hline 3 & 4 & 8 & 0 & 13 & 0 & 25 \\
\hline 4 & 0 & 7 & 12 & 0 & 0 & 19 \\
\hline 5 & 4 & 10 & 0 & 12 & 0 & 26 \\
\hline 6 & 5 & 12 & 0 & 11 & 0 & 28 \\
\hline 7 & 5 & 10 & 0 & 13 & 0 & 28 \\
\hline 8 & 0 & 6 & 12 & 0 & 0 & 18 \\
\hline 9 & 3 & 7 & 0 & 12 & 0 & 22 \\
\hline 10 & 5 & 9 & 0 & 15 & 0 & 29 \\
\hline
\end{tabular}

\begin{tabular}{|c|c|c|c|c|c|c|}
\hline Reproduction at & \multicolumn{4}{c|}{ Concentration } \\
\hline Rep. & Day 3/4 & Day 5 & Day 6 & Day 7 & Day 3 & Total \\
\hline 1 & 0 & 1 & 3 & 0 & 0 & 4 \\
\hline 2 & 5 & 6 & 15 & 0 & 0 & 26 \\
\hline 3 & $X$ & $X$ & $X$ & $X$ & $X$ & 0 \\
\hline 4 & 4 & 8 & 14 & 0 & 0 & 26 \\
\hline 5 & 5 & 13 & 0 & 14 & 0 & 32 \\
\hline 6 & 4 & 10 & 0 & 13 & 0 & 27 \\
\hline 7 & 4 & 8 & 0 & 16 & 0 & 28 \\
\hline 8 & 0 & 4 & 11 & 0 & 0 & 15 \\
\hline 9 & 2 & 0 & 8 & 10 & 0 & 20 \\
\hline 10 & 4 & 0 & 9 & 12 & 0 & 25 \\
\hline
\end{tabular}

County:

NPDES\#:

ETT \#:
Aiken

SC

19850

\begin{tabular}{|c|c|c|c|c|c|c|}
\hline \multicolumn{2}{|c|}{ Reproduction at } & \multicolumn{2}{c|}{$6.25 \%$} & \multicolumn{2}{c|}{ Concentration } \\
\hline Rep. & Day 3/4 & Day 5 & Day 6 & Day 7 & Day 8 & Total \\
\hline 1 & 3 & 0 & 9 & 13 & 0 & 25 \\
\hline 2 & 5 & 10 & 15 & 0 & 0 & 30 \\
\hline 3 & 5 & 8 & 0 & 15 & 0 & 28 \\
\hline 4 & 5 & 8 & 0 & 13 & 0 & 26 \\
\hline 5 & 4 & 5 & 0 & 11 & 0 & 20 \\
\hline 6 & 3 & 6 & 0 & 14 & 0 & 23 \\
\hline 7 & 3 & 10 & 0 & 14 & 0 & 27 \\
\hline 8 & 0 & 6 & 11 & 0 & 0 & 17 \\
\hline 9 & 4 & 0 & 9 & 13 & 0 & 26 \\
\hline 10 & 5 & 8 & 0 & 13 & 0 & 26 \\
\hline
\end{tabular}

\begin{tabular}{|c|c|c|c|c|c|c|}
\hline \multicolumn{2}{|c|}{ Reproduction at } & \multicolumn{3}{c|}{$25 \%$} & \multicolumn{2}{c|}{ Concentration } \\
\hline Rep. & Day 3/4 & Day 5 & Day 6 & Day 7 & Day 8 & Toual \\
\hline 1 & 0 & 8 & 0 & 12 & 0 & 20 \\
\hline 2 & 0 & 8 & 14 & 0 & 0 & 22 \\
\hline 3 & 3 & 7 & 0 & 13 & 0 & 23 \\
\hline 4 & 2 & $X$ & $X$ & $X$ & $X$ & 2 \\
\hline 5 & 4 & 9 & 0 & 15 & 0 & 28 \\
\hline 6 & 5 & 10 & 0 & 15 & 0 & 30 \\
\hline 7 & 4 & 9 & 0 & 14 & 0 & 27 \\
\hline 8 & 0 & 6 & 10 & 9 & 0 & 25 \\
\hline 9 & 0 & 7 & 0 & 10 & 0 & 17 \\
\hline 10 & 5 & 0 & 9 & 13 & 0 & 27 \\
\hline
\end{tabular}

\begin{tabular}{|c|c|c|c|c|c|c}
\hline \multicolumn{2}{|c|}{ Reproduction at } & \multicolumn{2}{c}{ 100\% } & \multicolumn{3}{c}{ Concentration } \\
\hline Rep. & Day 3/4 & Day 5 & Day 6 & Day 7 & Day 8 & Total \\
\hline 1 & 2 & 9 & 0 & 12 & 0 & 23 \\
\hline 2 & 0 & 6 & 17 & 0 & 0 & 23 \\
\hline 3 & 4 & 11 & 2 & 11 & 0 & 28 \\
\hline 4 & 4 & 6 & 0 & 12 & 0 & 22 \\
\hline 5 & 4 & 10 & 0 & 11 & 0 & 25 \\
\hline 6 & 5 & 10 & 0 & 14 & 0 & 29 \\
\hline 7 & 2 & 0 & 9 & 12 & 0 & 23 \\
\hline 8 & 0 & 4 & 7 & 0 & 0 & 11 \\
\hline 9 & 4 & 0 & 8 & 0 & 0 & 12 \\
\hline 10 & 5 & 7 & 0 & 14 & 0 & 26 \\
\hline
\end{tabular}




\section{CHRONIC DEFINITIVE SURVIVAL AND REPRODUCTION TEST \\ Statistical Analyses}
Client:
WSRC/ G-10
Sample Identification:
G-10
Test Date:
May 14, 2002

\begin{tabular}{|lll|}
\hline Test for Normality & & \\
Test Used: Kolmogorov D & $\begin{array}{lll} \\
\end{array}$ & NA \\
& critical & NA
\end{tabular}

\begin{tabular}{|lr|}
\hline Test for Homogeneity of Variance \\
Test Used: Bartlett's Test & B \\
NA NA & \\
\end{tabular}

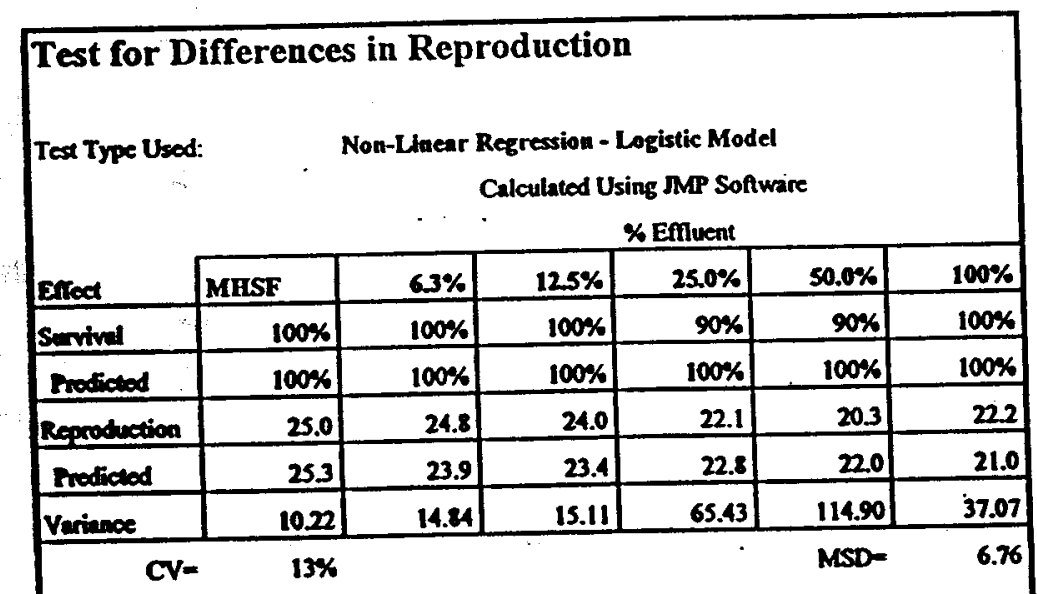

\begin{tabular}{|c|c|c|c|c|c|c|}
\hline \multicolumn{4}{|c|}{ Tex Parnmeters } & \multicolumn{2}{|c|}{ \% Reduction @ CTC } & \multirow[b]{2}{*}{ Paserfall } \\
\hline & alpha & beta & gamma & $19 \%$ & $100 \%$ & \\
\hline Survivel & 3.00 & -200.00 & 0.00 & $0.0 \%$ & $0.0 \%$ & Pass \\
\hline \multirow[t]{2}{*}{ Reproduction } & 26.00 & -196.00 & 24.30 & $8.8 \%$ & $16.9 \%$ & Pass \\
\hline & $\begin{array}{l}1 C 25= \\
\text { NOEC= }\end{array}$ & $\begin{array}{l}>100 \% \\
>100 \%\end{array}$ & & & & \\
\hline
\end{tabular}

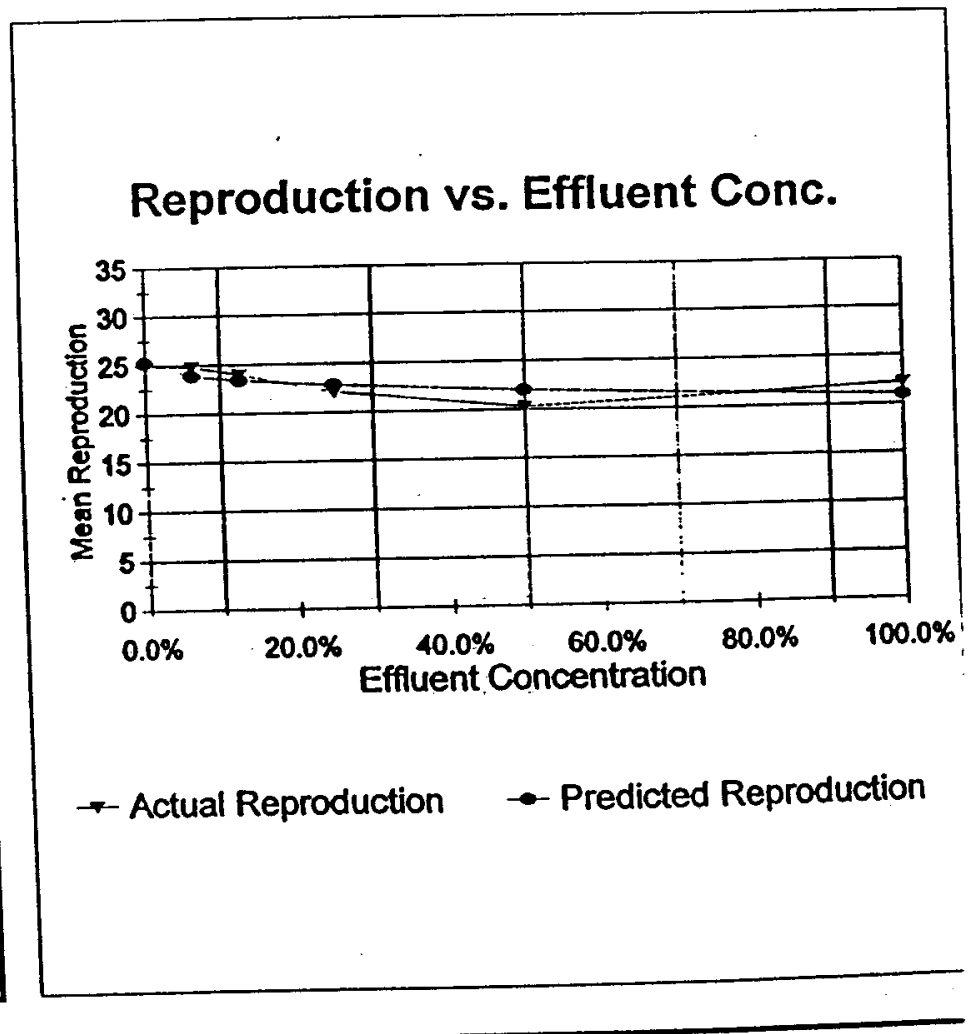

Summary

The reductions in survival and reproduction at the CTC are $<25 \%$ - Pass 


\section{CHRONIC DEFINITIVE SURVIVAL AND REPRODUCTION TEST \\ Water Chemistry}

Client: $\quad$ WSRC/ G-10

Sample Identification: G-10

Test Date: May 14,2002

\section{pH (After Renewal)}

\begin{tabular}{|l|r|r|r|r|r|r|}
\hline \multicolumn{7}{|c|}{ Test Concentration } \\
\hline & Control & $6.25 \%$ & $12.5 \%$ & $25.0 \%$ & $50.0 \%$ & $100 \%$ \\
\hline Initial & 7.02 & 7.00 & 7.06 & 7.15 & 7.31 & 7.48 \\
\hline Day 1 & 6.79 & 6.84 & 6.94 & 7.11 & 7.38 & 7.59 \\
\hline Day 2 & 7.20 & 7.08 & 7.07 & 7.18 & 7.40 & 7.58 \\
\hline Day 3 & 7.37 & 7.17 & 7.14 & 7.22 & 7.36 & 7.58 \\
\hline Day 4 & 7.39 & 7.03 & 7.11 & 7.28 & 7.39 & 7.39 \\
\hline Day 5 & 7.45 & 7.09 & 7.15 & 7.31 & 7.42 & 7.41 \\
\hline Day 6 & 7.91 & 7.24 & 7.13 & 7.20 & 7.33 & 7.44 \\
\hline
\end{tabular}

\section{Dissolved Oxygen (After Renewal)}

\begin{tabular}{|l|r|r|r|r|r|r|}
\hline & \multicolumn{7}{|c|}{ Test Concentration } \\
\hline Initial & 8.1 & 8.1 & 8.1 & 8.2 & 8.2 & 8.2 \\
\hline Day 1 & 8.1 & 8.1 & 8.2 & 8.3 & 8.3 & 8.2 \\
\hline Day 2 & 8.0 & 8.3 & 8.4 & 8.6 & 8.6 & 8.6 \\
\hline Day 3 & 8.2 & 8.2 & 8.2 & 8.3 & 8.3 & 8.4 \\
\hline Day 4 & 8.1 & 8.1 & 8.1 & 8.3 & 8.2 & 8.3 \\
\hline Day 5 & 8.1 & 8.1 & 8.2 & 8.2 & 8.3 & 8.4 \\
\hline Day 6 & 8.1 & 8.2 & 8.2 & 8.3 & 8.3 & 8.4 \\
\hline
\end{tabular}

Test Results Reviewed and Approved By:
$\mathrm{pH}$ (Before Renewal)

\begin{tabular}{|l|r|r|r|r|r|r|}
\hline & Control & $6.25 \%$ & $12.5 \%$ & $25.0 \%$ & $50.0 \%$ & $100 \%$ \\
\hline Day 1 & 6.80 & 6.81 & 7.10 & 7.16 & 7.41 & 7.62 \\
\hline Day 2 & 7.30 & 7.22 & 7.08 & 7.25 & 7.38 & 7.76 \\
\hline Day 3 & 7.54 & 7.42 & 7.34 & 7.38 & 7.58 & \\
\hline Day 4 & 7.45 & 7.36 & 7.27 & 4.14 & 7.23 & 7.31 \\
\hline Day 5 & 7.53 & 7.43 & 7.31 & 7.20 & 7.25 & 7.32 \\
\hline Day 6 & 7.56 & 7.54 & 7.45 & 7.40 & 7.51 & 7.63 \\
\hline Final & 7.14 & 7.31 & 7.10 & 7.08 & 7.42 & 7.58 \\
\hline
\end{tabular}

Dissolved Oxygen (Before Renewal)

\begin{tabular}{|l|r|r|r|r|r|r}
\hline & \multicolumn{7}{|c|}{ Test Concentration } \\
\hline Day 1 & 8.2 & 8.2 & 5.3 & 8.3 & 8.3 & 8.3 \\
\hline Day 2 & 8.1 & 8.1 & 8.2 & 8.3 & 8.4 & 8.4 \\
\hline Day 3 & 8.1 & 8.0 & 8.0 & 8.0 & 8.0 & \\
\hline Day 4 & 8.0 & 8.1 & 8.1 & 8.2 & 8.2 & 8.3 \\
\hline Day 5 & 8.1 & 8.2 & 8.3 & 8.4 & 8.4 & 8.6 \\
\hline Day 6 & 8.2 & 8.3 & 8.4 & 8.4 & 8.5 & 8.5 \\
\hline Final & 8.4 & 8.4 & 8.4 & 8.4 & 8.3 & 8.2 \\
\hline
\end{tabular}

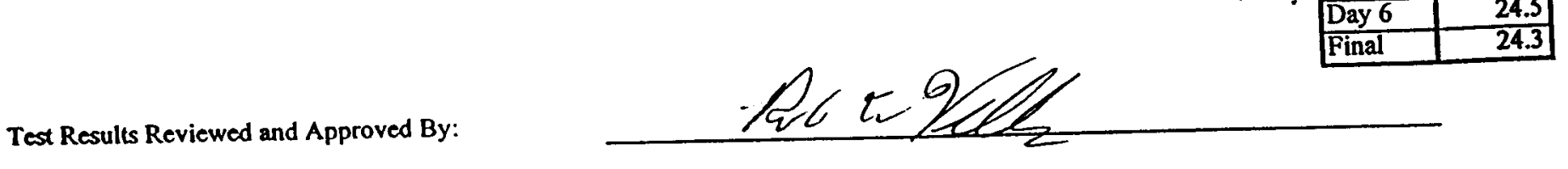




\section{Day Chronic Definitive Survival and Reproduction Bioassay \\ Method: EPA/600/4-91/002}

Test Organism:

Daphnia ambigua

Facility: WSRC

Sample ID: A-01

Aiken County

ETT\#: 19985

Sample ID: June 4, 2002 


\section{CHRONIC DEFINITIVE SURVIVAL AND REPRODUCTION TEST}

\section{Survival and Reproduction Results}

Client: WSRC

Sample Identification: A-01

Test Date:

June 4, 2002

$\begin{array}{ll}\text { County: } & \text { Aiken } \\ \text { NPDESH: } & \text { SC }\end{array}$

ETT \#:
19985

\begin{tabular}{|c|c|c|c|c|c|c|}
\hline Reproduction at & \multicolumn{7}{|c|}{ Control } \\
\hline Rep. & Day 3/4 & Day 5 & Day 6 & Day 7 & Day 8 & Total \\
\hline 1 & 0 & 6 & 8 & 0 & 12 & 26 \\
\hline 2 & 0 & 6 & 10 & 0 & 8 & 24 \\
\hline 3 & 0 & 4 & 10 & 0 & 14 & 28 \\
\hline 4 & 0 & 5 & 12 & 0 & 6 & 23 \\
\hline 5 & 0 & 4 & 8 & $X$ & $X$ & 12 \\
\hline 6 & 0 & 5 & 13 & 0 & 8 & 26 \\
\hline 7 & 0 & 3 & 9 & 0 & 10 & 22 \\
\hline 8 & 0 & 3 & 11 & 0 & 6 & 20 \\
\hline 9 & 0 & 5 & 0 & 11 & 2 & 18 \\
\hline 10 & 0 & 6 & $X$ & $X$ & $X$ & 6 \\
\hline
\end{tabular}

\begin{tabular}{|c|c|c|c|c|c|c|}
\hline \multicolumn{2}{|c|}{ Reproduction at } & \multicolumn{2}{c|}{$6.25 \%$} & \multicolumn{2}{c|}{ Concentration } \\
\hline Rep. & Day 3/4 & Day 5 & Day 6 & Day 7 & Day 8 & Total \\
\hline 1 & 0 & 4 & $\mathrm{X}$ & $\mathrm{X}$ & $\mathrm{X}$ & 4 \\
\hline 2 & 0 & 6 & 10 & 0 & 12 & 28 \\
\hline 3 & 0 & 3 & 9 & 0 & 9 & 21 \\
\hline 4 & $\mathrm{~L}$ & $\mathrm{~L}$ & $\mathrm{~L}$ & $\mathrm{~L}$ & $\mathrm{~L}$ & $\mathrm{~L}$ \\
\hline 5 & 3 & 0 & 6 & 12 & 0 & 21 \\
\hline 6 & 0 & 5 & 10 & 0 & 14 & 29 \\
\hline 7 & 0 & 5 & 11 & 0 & 0 & 16 \\
\hline 8 & 0 & 4 & 10 & 0 & 8 & 22 \\
\hline 9 & 5 & 0 & 8 & 8 & 0 & 21 \\
\hline 10 & 0 & 5 & 7 & 0 & 14 & 26 \\
\hline
\end{tabular}

\begin{tabular}{|c|c|c|c|c|c|c|}
\hline \multicolumn{2}{|c|}{ Reproduction at } & \multicolumn{3}{c|}{$12.5 \%$} & \multicolumn{3}{c|}{ Concentration } \\
\hline Rep. & Day 3/4 & Day 5 & Day 6 & Day 7 & Day 8 & Total \\
\hline I & male & $L$ & $L$ & $L$ & $L$ & $L$ \\
\hline 2 & 0 & 7 & 12 & 2 & $X$ & 21 \\
\hline 3 & male & $L$ & $L$ & $L$ & $L$ & $L$ \\
\hline 4 & 0 & 7 & $X$ & $X$ & $X$ & 7 \\
\hline 5 & 0 & 4 & 9 & 0 & 5 & 18 \\
\hline 6 & 0 & 5 & 12 & 0 & 14 & 31 \\
\hline 7 & 0 & 5 & 0 & 14 & 10 & 29 \\
\hline 8 & 0 & 4 & $X$ & $X$ & $X$ & 4 \\
\hline 9 & 3 & 0 & 8 & 0 & 10 & 21 \\
\hline 10 & 0 & .6 & 11 & 0 & 11 & 28 \\
\hline
\end{tabular}

\begin{tabular}{|c|c|c|c|c|c|c|}
\hline \multicolumn{2}{|c|}{ Reproduction at } & \multicolumn{3}{c|}{$25 \%$} & \multicolumn{2}{c|}{ Concentration } \\
\hline Rep. & Day 3/4 & Day 5 & Day 6 & Day 7 & Day 8 & Total \\
\hline 1 & 0 & 4 & 10 & 0 & 11 & 25 \\
\hline 2 & 0 & 7 & 12 & 0 & 11 & 30 \\
\hline 3 & 3 & 0 & 10 & 12 & 0 & 25 \\
\hline 4 & 0 & 5 & 7 & 0 & 10 & 22 \\
\hline 5 & male & $L$ & $\mathrm{~L}$ & $\mathrm{~L}$ & $\mathrm{~L}$ & $\mathrm{~L}$ \\
\hline 6 & 0 & 5 & 9 & 0 & 15 & 29 \\
\hline 7 & 0 & 4 & 0 & 9 & 13 & 26 \\
\hline 8 & 0 & 5 & 11 & 0 & 12 & 28 \\
\hline 9 & 0 & 4 & 11 & 0 & 14 & 29 \\
\hline 10 & 0 & 6 & 12 & 0 & 11 & 29 \\
\hline
\end{tabular}

\begin{tabular}{|c|c|c|c|c|c|c|}
\hline Reproduction at & \multicolumn{4}{|c|}{$50 \%$} & \multicolumn{3}{c|}{ Concentration } \\
\hline Rep. & Day 3/4 & Day 5 & Day 6 & Day 7 & Day & Total \\
\hline 1 & 0 & X & X & X & X & 0 \\
\hline 2 & 0 & 6 & 14 & 10 & 0 & 30 \\
\hline 3 & male & L & L & L & L & L \\
\hline 4 & 0 & 5 & 8 & 0 & 16 & 29 \\
\hline 5 & male & L & L & L & L & L \\
\hline 6 & 0 & 4 & 11 & 0 & 14 & 29 \\
\hline 7 & 0 & 5 & 11 & 0 & 14 & 30 \\
\hline 8 & 0 & 5 & 9 & 0 & 5 & 19 \\
\hline 9 & 4 & 0 & 0 & 7 & 0 & 20 \\
\hline 10 & 0 & 4 & 12 & 0 & 13 & 29 \\
\hline
\end{tabular}

\begin{tabular}{|c|c|c|c|c|c|c}
\hline \multicolumn{2}{|c}{ Reproduction at } & \multicolumn{2}{c}{$100 \%$} & \multicolumn{2}{c}{ Concentration } \\
\hline Rep. & Day 3/4 & Day 5 & Day 6 & Day 7 & Day 8 & Total \\
\hline 1 & 0 & 4 & 8 & 0 & 15 & 27 \\
\hline 2 & 0 & 5 & 8 & 11 & 0 & 24 \\
\hline 3 & male & $\mathrm{L}$ & $\mathrm{L}$ & $\mathrm{L}$ & $\mathrm{L}$ & $\mathrm{L}$ \\
\hline 4 & 0 & 4 & 8 & 0 & 15 & 27 \\
\hline 5 & male & $\mathrm{L}$ & $\mathrm{L}$ & $\mathrm{L}$ & $\mathrm{L}$ & $\mathrm{L}$ \\
\hline 6 & 0 & 5 & 12 & 0 & 6 & 23 \\
\hline 7 & 0 & 4 & 13 & 0 & 16 & 33 \\
\hline 8 & 0 & 4 & 12 & 0 & 15 & 31 \\
\hline 9 & 0 & $\mathrm{X}$ & $\mathrm{X}$ & $\mathrm{X}$ & $\mathrm{X}$ & 0 \\
\hline 10 & 0 & 3 & 14 & 0 & 16 & 33 \\
\hline
\end{tabular}




\section{CHRONIC DEFINITIVE SURVIVAL AND REPRODUCTION TEST \\ Statistical Analyses}

Client:

WSRC

Sample Identification:

Test Date:

A-01

June 4, 2002

\section{Test for Normality}

Test Used: Shapiro-Wilks

$\begin{array}{ll}W= & N A \\ \text { critical } & \text { NA }\end{array}$

NA

\section{Test for Differences in Reproduction}

\begin{tabular}{|c|c|c|c|c|c|c|}
\hline \multicolumn{2}{|c|}{ Teat Type Used: } & \multicolumn{5}{|c|}{$\begin{array}{l}\text { Non-Linear Regression - Logistic Model } \\
\text { Calculated Using MMP Software }\end{array}$} \\
\hline Entect & MHSF & $6.3 \%$ & $12.5 \%$ & $25.0 \%$ & $50.0 \%$ & $100 \%$ \\
\hline Survival & $80 \%$ & $88 \%$ & $63 \%$ & $100 \%$ & 88\% & $88 \%$ \\
\hline Prodictod & $100 \%$ & $100 \%$ & $100 \%$ & $100 \%$ & $100 \%$ & $100 \%$ \\
\hline Repeoduction & 20.5 & 20.9 & 19.9 & 27.0 & 23.3 & 24.8 \\
\hline Inedictod & 22.7 & 22.7 & 22.7 & 22.7 & 22.7 & 227 \\
\hline Variance & 47,39 & 56.61 & 99.55 & 2.00 & 108.50 & 114.50 \\
\hline $\mathrm{CV}=$ & $34 \%$ & & & & $M S D=$ & 8.59 \\
\hline
\end{tabular}

Text Paramelers

\begin{tabular}{|c|c|c|c|c|c|}
\hline \multicolumn{6}{|c|}{ 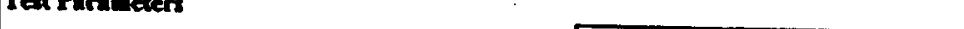 } \\
\hline & alpha & beta & Eamma & $\begin{array}{l}\text { \% Reduction } \\
\text { CTC }=93.4 \%\end{array}$ & Paser/Fal \\
\hline Survival & 3.00 & -200.00 & 0.00 & $0.0 \%$ & Pass \\
\hline Reproduction & 4.78 & -20.00 & 22.67 & $0.0 \%$ & Pess \\
\hline & IC25 $=$ & $>100 \%$ & & & \\
\hline
\end{tabular}

Summary

The reductions in survival and reproduction at the CTC are $<25 \%$ - Pass

\section{Test for Homogeneity of Variance}

Test Used: Bartlett's Test

$B=N A$

critical NA

\section{NA}

Reproduction vs. Effluent Conc.

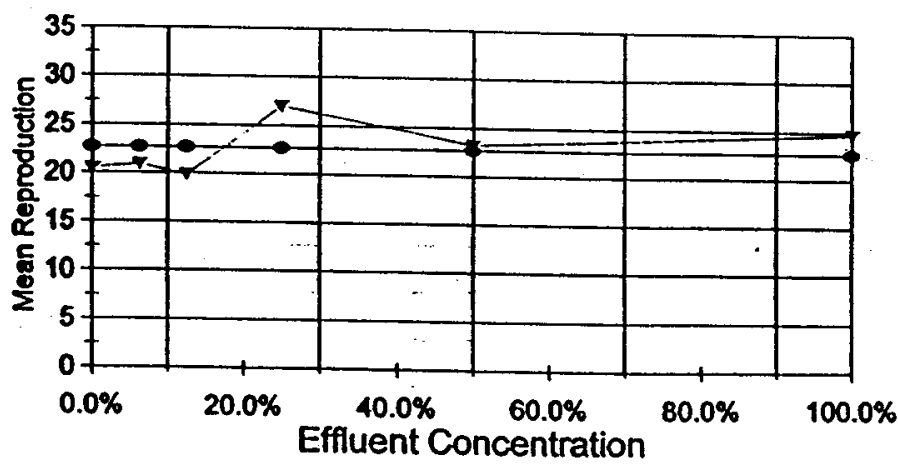

Actual Reproduction 


\section{CHRONIC DEFINITIVE SURVIVAL AND REPRODUCTION TEST \\ Water Chemistry}

Client: WSRC

Sample Identification: $\quad$ A-01

Test Date: June 4, 2002

pH (After Renewal)

\begin{tabular}{|l|r|r|r|r|r|r|}
\hline \multicolumn{7}{|c|}{ Test Concentration } \\
\hline & control & $6.25 \%$ & $12.5 \%$ & $25 \%$ & $50 \%$ & $100 \%$ \\
\hline Initial & 7.21 & 7.03 & 6.95 & 6.90 & 6.88 & 6.79 \\
\hline Day 1 & 6.82 & 6.81 & 6.80 & 6.82 & 6.83 & 6.91 \\
\hline Day 2 & 6.92 & 6.90 & 6.84 & 6.90 & 7.00 & 7.07 \\
\hline Day 3 & 7.40 & 7.27 & 7.24 & 7.15 & 7.11 & 7.05 \\
\hline Day 4 & 7.00 & 6.95 & 6.93 & 6.90 & 7.01 & 7.03 \\
\hline Day 5 & 7.33 & 7.09 & 7.00 & 6.94 & 6.90 & 6.83 \\
\hline Day 6 & 6.96 & 7.25 & 6.97 & 6.97 & 6.82 & 6.77 \\
\hline
\end{tabular}

Dissolved Oxygen (After Renewal)

\begin{tabular}{|l|r|r|r|r|r|r|}
\hline & \multicolumn{7}{|c|}{ Test Concentration } \\
\hline Lnitial & 8.1 & 8.0 & 8.0 & 8.0 & 8.0 & 7.9 \\
\hline Day 1 & 8.0 & 8.1 & 8.2 & 8.2 & 8.2 & 8.3 \\
\hline Day 2 & 8.1 & 8.0 & 8.0 & 8.0 & 8.1 & 8.1 \\
\hline Day 3 & 8.2 & 8.1 & 8.1 & 8.1 & 8.1 & 8.2 \\
\hline Day 4 & 8.3 & 8.1 & 8.1 & 8.1 & 8.1 & 8.1 \\
\hline Day 5 & 8.4 & 8.4 & 8.3 & 8.3 & 8.3 & 8.1 \\
\hline Day 6 & 8.4 & 8.4 & 8.5 & 8.5 & 8.5 & 8.5 \\
\hline
\end{tabular}

Test Results Reviewed and Approved By:
pH (Before Renewal)

\begin{tabular}{|l|r|r|r|r|r|r|}
\hline \multicolumn{7}{|c|}{ Test Concentration } \\
\hline & control & $6.25 \%$ & $12.5 \%$ & $25 \%$ & $50 \%$ & $100 \%$ \\
\hline Day 1 & 6.82 & 6.84 & 6.83 & 6.85 & 6.90 & 7.04 \\
\hline Day 2 & 7.15 & 7.11 & 7.11 & 7.11 & 7.13 & 7.20 \\
\hline Day 3 & 7.45 & 7.49 & 7.27 & 7.22 & 7.20 & 7.23 \\
\hline Day 4 & 7.20 & 7.15 & 7.13 & 7.13 & 7.23 & 7.25 \\
\hline Day 5 & 7.38 & 7.56 & 7.38 & 7.09 & 7.00 & 6.99 \\
\hline Day 6 & 6.78 & 6.90 & 6.88 & 6.94 & 7.03 & 7.00 \\
\hline Final & 7.31 & 7.63 & 7.60 & 7.50 & 7.41 & 7.42 \\
\hline
\end{tabular}

Dissolved Oxygen (Before Renewal)

\begin{tabular}{|l|r|r|r|r|r|r|}
\hline \multicolumn{7}{|c|}{ Test Concentration } \\
\hline & control & $6.25 \%$ & $12.5 \%$ & $25 \%$ & $50 \%$ & $100 \%$ \\
\hline Day 1 & 6.8 & 7.0 & 7.2 & 7.0 & 7.0 & 7.2 \\
\hline Day 2 & 7.9 & 8.0 & 8.0 & 8.2 & 8.3 & 8.3 \\
\hline Day 3 & 8.1 & 8.0 & 8.0 & 8.1 & 8.1 & 8.2 \\
\hline Day 4 & 8.1 & 8.0 & 8.1 & 8.1 & 8.1 & 8.2 \\
\hline Day 5 & 8.2 & 8.0 & 8.0 & 8.0 & 8.0 & 8.0 \\
\hline Day 6 & 8.4 & 8.1 & 8.5 & 8.5 & 8.4 & 8.4 \\
\hline Final & 8.1 & 8.0 & 7.9 & 7.8 & 7.8 & 7.6 \\
\hline
\end{tabular}

Temperature

\begin{tabular}{|l|r|}
\hline \multicolumn{2}{|c|}{ Incubator ${ }^{\circ} \mathrm{C}$} \\
\hline Initial & 25.1 \\
\hline Day 1 & 24.3 \\
\hline Day 2 & 24.5 \\
\hline Day 3 & 24.4 \\
\hline Day 4 & 24.5 \\
\hline Day 5 & 24.2 \\
\hline Day 6 & 24.4 \\
\hline Final & 24.5 \\
\hline
\end{tabular}




\section{Day Chronic Definitive Survival and Reproduction Bioassay \\ Method: EPA/600/4-91/002}

Test Organism:

Daphnia ambigua

Facility: WSRC

Sample ID: A-11

Aiken County

ETT\#: 19986

Sample ID: June 4, 2002 


\section{CHRONIC DEFINITIVE SURVIVAL AND REPRODUCTION TEST \\ Survival and Reproduction Results}

Client:

Sample Identification:

Test Date:

WSRC

A-11

June 4, 2002
County:

NPDES\#:

ETT \#:
Aiken

$\mathrm{SC}$

19986

\begin{tabular}{|c|c|c|c|c|c|c|}
\hline Reproduction at & \multicolumn{7}{c|}{ Control } \\
\hline Rep. & Day 3/4 & Day 5 & Day 6 & Day 7 & Day 8 & Total \\
\hline 1 & 0 & 6 & 8 & 0 & 12 & 26 \\
\hline 2 & 0 & 6 & 10 & 0 & 8 & 24 \\
\hline 3 & 0 & 4 & 10 & 0 & 14 & 28 \\
\hline 4 & 0 & 5 & 12 & 0 & 6 & 23 \\
\hline 5 & 0 & 4 & 8 & $X$ & $X$ & 12 \\
\hline 6 & 0 & 5 & 13 & 0 & 8 & 26 \\
\hline 7 & 0 & 3 & 9 & 0 & 10 & 22 \\
\hline 8 & 0 & 3 & 11 & 0 & 6 & 20 \\
\hline 9 & 0 & 5 & 0 & 11 & 2 & 18 \\
\hline 10 & 0 & 6 & $X$ & $X$ & $X$ & 6 \\
\hline
\end{tabular}

\begin{tabular}{|c|c|c|c|c|c|c|}
\hline \multicolumn{2}{|c|}{ Reproduction at } & \multicolumn{2}{c|}{$6.25 \%$} & \multicolumn{2}{c|}{ Concentration } \\
\hline Rep. & Day 3/4 & Day 5 & Day 6 & Day 7 & Day 8 & Total \\
\hline 1 & 0 & 5 & 8 & 0 & 9 & 22 \\
\hline 2 & 0 & 0 & 0 & 3 & 4 & 7 \\
\hline 3 & male & $\mathrm{L}$ & $\mathrm{L}$ & $\mathrm{L}$ & $\mathrm{L}$ & $\mathrm{L}$ \\
\hline 4 & 0 & 4 & 12 & $\mathrm{X}$ & $\mathrm{X}$ & 16 \\
\hline 5 & male & $\mathrm{L}$ & $\mathrm{L}$ & $\mathrm{L}$ & $\mathrm{L}$ & $\mathrm{L}$ \\
\hline 6 & 0 & 5 & 8 & 0 & 15 & 28 \\
\hline 7 & 0 & 5 & 11 & 0 & 15 & 31 \\
\hline 8 & 0 & 4 & 9 & 0 & 11 & 24 \\
\hline 9 & 4 & 0 & 9 & 12 & 0 & 25 \\
\hline 10 & 0 & 5 & 9 & 0 & 12 & 26 \\
\hline
\end{tabular}

\begin{tabular}{|c|c|c|c|c|c|c|}
\hline \multicolumn{1}{|c|}{ Reproduction at } & \multicolumn{4}{c|}{$12.5 \%$} & \multicolumn{3}{c|}{ Concentration } \\
\hline Rep. & Day 3/4 & Day 5 & Day 6 & Day 7 & Day 8 & Total \\
\hline 1 & 0 & 5 & 10 & 11 & 0 & 26 \\
\hline 2 & 0 & 7 & 13 & X & X & 20 \\
\hline 3 & male & $L$ & $\mathrm{~L}$ & $\mathrm{~L}$ & $\mathrm{~L}$ & $\mathrm{~L}$ \\
\hline 4 & 0 & 4 & 7 & 0 & 5 & 16 \\
\hline 5 & 0 & 6 & 10 & 10 & 0 & 26 \\
\hline 6 & 0 & 4 & 10 & 0 & 13 & 27 \\
\hline 7 & 0 & 6 & 14 & 0 & 13 & 33 \\
\hline 8 & 0 & 5 & 11 & $X$ & $X$ & 16 \\
\hline 9 & 4 & 0 & 9 & 12 & 0 & 25 \\
\hline 10 & 0 & 5 & 10 & 0 & 10 & 25 \\
\hline
\end{tabular}

\begin{tabular}{|c|c|c|c|c|c|c|}
\hline \multicolumn{2}{|c|}{ Reproduction at } & \multicolumn{2}{c|}{$25 \%$} & \multicolumn{2}{c|}{ Concentration } \\
\hline Rep. & Day 3/4 & Day 5 & Day 6 & Day 7 & Day 8 & Toul \\
\hline 1 & 0 & 5 & 13 & 0 & 12 & 30 \\
\hline 2 & 0 & 4 & 8 & 0 & 15 & 27 \\
\hline 3 & male & $\mathrm{L}$ & $\mathrm{L}$ & $\mathrm{L}$ & $\mathrm{L}$ & $\mathrm{L}$ \\
\hline 4 & 0 & 4 & 10 & 0 & 12 & 26 \\
\hline 5 & 0 & 5 & 12 & 0 & 11 & 28 \\
\hline 6 & 0 & 5 & 10 & 0 & 14 & 29 \\
\hline 7 & 0 & 4 & 8 & 0 & 14 & 26 \\
\hline 8 & 0 & 3 & 13 & 1 & $X$ & 17 \\
\hline 9 & 4 & 0 & 11 & 12 & 0 & 27 \\
\hline 10 & 0 & 4 & 9 & 0 & 9 & 22 \\
\hline
\end{tabular}

\begin{tabular}{|c|c|c|c|c|c|c|}
\hline Reproduction at & \multicolumn{4}{c|}{$50 \%$} & \multicolumn{3}{c|}{ Concentration } \\
\hline Rep. & Day 3/4 & Day 5 & Day 6 & Day 7 & Day 8 & Total \\
\hline 1 & 0 & 4 & 8 & 10 & 0 & 22 \\
\hline 2 & 0 & 7 & 10 & 0 & 9 & 26 \\
\hline 3 & 0 & 5 & 8 & 0 & 4 & 17 \\
\hline 4 & 0 & 6 & 10 & 0 & 16 & 32 \\
\hline 5 & male & $\mathrm{L}$ & $\mathrm{L}$ & $\mathrm{L}$ & $\mathrm{L}$ & $\mathrm{L}$ \\
\hline 6 & 0 & 6 & 11 & 0 & 4 & 21 \\
\hline 7 & 0 & 6 & 12 & 0 & 11 & 29 \\
\hline 8 & 0 & 6 & 11 & 0 & 10 & 27 \\
\hline 9 & 4 & 0 & 10 & 12 & 0 & 26 \\
\hline 10 & 0 & 3 & 11 & 0 & 6 & 20 \\
\hline
\end{tabular}

\begin{tabular}{|c|c|c|c|c|c|c}
\hline \multicolumn{2}{|c|}{ Reproduction at } & \multicolumn{2}{c}{$100 \%$} & \multicolumn{2}{c}{ Concentration } \\
\hline Rep. & Day 3/4 & Day 5 & Day 6 & Day 7 & Day 8 & Total \\
\hline 1 & 0 & 4 & 10 & 0 & 9 & 23 \\
\hline 2 & 0 & 5 & 12 & 0 & 6 & 23 \\
\hline 3 & male & $\mathrm{L}$ & $\mathrm{L}$ & $\mathrm{L}$ & $\mathrm{L}$ & $\mathrm{L}$ \\
\hline 4 & 0 & 7 & 11 & $\mathrm{X}$ & $\mathrm{X}$ & 18 \\
\hline 5 & male & $\mathrm{L}$ & $\mathrm{L}$ & $\mathrm{L}$ & $\mathrm{L}$ & $\mathrm{L}$ \\
\hline 6 & 0 & 4 & 8 & 0 & 11 & 23 \\
\hline 7 & 0 & 4 & 13 & 0 & 5 & 22 \\
\hline 8 & 0 & 5 & 10 & 0 & 13 & 28 \\
\hline 9 & 4 & 0 & 10 & 4 & 0 & 18 \\
\hline 10 & 0 & 3 & 10 & 0 & 12 & 25 \\
\hline
\end{tabular}




\section{CHRONIC DEFINITIVE SURVIVAL AND REPRODUCTION TEST \\ Statistical Analyses}

Client:

WSRC

Sample Identification:

Test Date:

June 4, 2002

\begin{tabular}{|lll|}
\hline Test for Normality & & \\
& & \\
Test Used: Shapiro-Wilks & W= & NA \\
& critical & NA
\end{tabular}

Test for Homogeneity of Variance

Test Used: Bartlett's Test

$B=N A$

critical NA

NA

NA

Reproduction vs. Effluent Conc.

\begin{tabular}{|c|c|c|c|c|c|c|}
\hline \multirow[b]{2}{*}{ Enfect } & \multicolumn{6}{|c|}{$\%$ Effluent } \\
\hline & MHSF & $6.3 \%$ & $125 \%$ & $25.0 \%$ & $50.0 \%$ & $100 \%$ \\
\hline Survival & $20 x$ & $100 \%$ & $78 \%$ & $89 \%$ & $100 \%$ & $88 \%$ \\
\hline Prodicted & $100 \%$ & $100 \%$ & $100 \%$ & $100 \%$ & $100 \%$ & $100 \%$ \\
\hline Reproduction & 20.5 & 22.4 & 23.8 & 25.8 & 24.4 & 22.5 \\
\hline Predicted & 23.2 & 23.2 & 23.2 & 23.2 & 23.2 & 23.2 \\
\hline Variance & 47.39 & 57.98 & 30.44 & 15.94 & 22.78 & 11.14 \\
\hline$y=$ & $34 \%$ & & & & MSD & 5.75 \\
\hline
\end{tabular}

Text Parameters

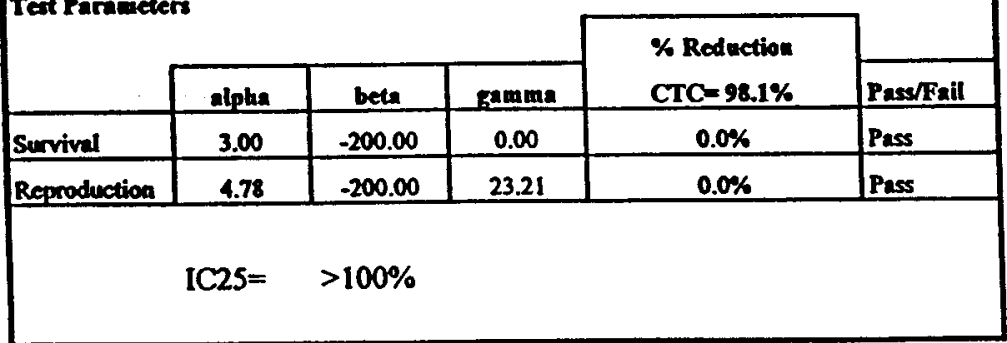

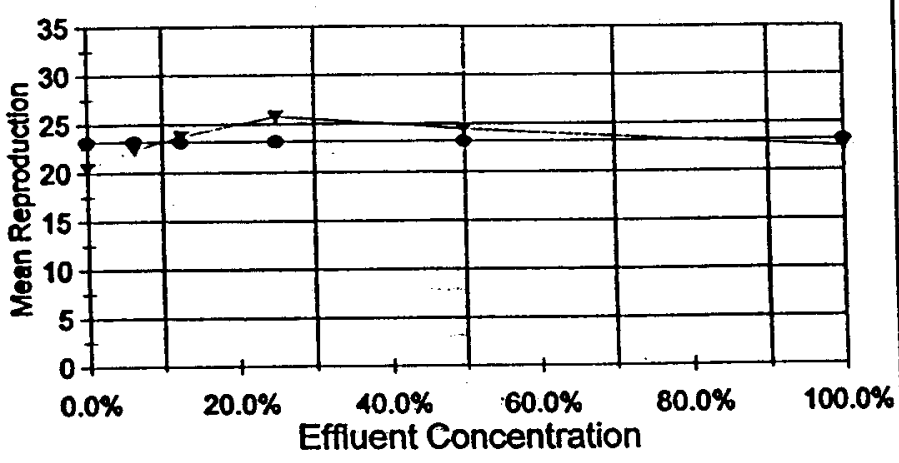

$\rightarrow$ Actual Reproduction $\quad \bullet$ Predicted Reproduction

Summary

The reductions in survival and reproduction at the CTC are $<25 \%$ - Pass 


\section{CHRONIC DEFINITIVE SURVIVAL AND REPRODUCTION TEST \\ Water Chemistry}

$\begin{array}{ll}\text { Client: } & \text { WSRC } \\ \text { Sample Identification: } & \text { A-11 } \\ \text { Test Date: } & \text { June 4, } 2002\end{array}$

pH (After Renewal)

\begin{tabular}{|l|r|r|r|r|r|r|}
\hline \multicolumn{7}{|c|}{ Test Concentration } \\
\hline & control & $6.25 \%$ & $12.5 \%$ & $25 \%$ & $50 \%$ & $100 \%$ \\
\hline Initial & 7.21 & 7.28 & 7.15 & 7.11 & 7.09 & 7.13 \\
\hline Day 1 & 6.82 & 6.87 & 6.81 & 6.80 & 6.81 & 6.87 \\
\hline Day 2 & 6.92 & 7.04 & 7.00 & 6.99 & 6.93 & 6.96 \\
\hline Day 3 & 7.40 & 7.00 & 7.00 & 6.94 & 6.91 & 6.83 \\
\hline Day 4 & 7.00 & 7.05 & 7.03 & 7.00 & 6.95 & 6.93 \\
\hline Day 5 & 7.33 & 6.99 & 6.94 & 6.83 & 6.81 & 6.80 \\
\hline Day 6 & 6.96 & 8.02 & 8.42 & 8.33 & 8.29 & 8.07 \\
\hline
\end{tabular}

Dissolved Oxygen (After Renewal)

\begin{tabular}{|l|r|r|r|r|r|r|}
\hline & \multicolumn{7}{|c|}{ Test Concentration } \\
\hline Initial & 8.1 & 8.5 & 8.3 & 8.2 & 8.1 & 8.0 \\
\hline Day 1 & 8.0 & 8.6 & 8.6 & 8.6 & 8.6 & 8.6 \\
\hline Day 2 & 8.1 & 8.6 & 8.6 & 8.7 & 8.6 & 8.6 \\
\hline Day 3 & 8.2 & 8.5 & 8.5 & 8.4 & 8.4 & 8.4 \\
\hline Day 4 & 8.3 & 8.4 & 8.4 & 8.5 & 8.6 & 8.6 \\
\hline Day 5 & 8.4 & 8.5 & 8.5 & 8.5 & 8.6 & 8.7 \\
\hline Day 6 & 8.4 & 8.5 & 8.5 & 8.5 & 8.5 & 8.5 \\
\hline
\end{tabular}

pH (Before Renewal)
\begin{tabular}{|l|r|r|r|r|r|r|}
\hline & \multicolumn{7}{|c|}{ Test Concentration } \\
\hline & control & $6.25 \%$ & $12.5 \%$ & $25 \%$ & $50 \%$ & $100 \%$ \\
\hline Day 1 & 6.82 & 6.95 & 6.90 & 6.88 & 6.88 & 6.90 \\
\hline Day 2 & 7.15 & 7.26 & 7.23 & 7.23 & 7.22 & 7.24 \\
\hline Day 3 & 7.45 & 7.00 & 6.95 & 6.91 & 6.83 & 6.89 \\
\hline Day 4 & 7.20 & 7.31 & 7.25 & 7.25 & 7.20 & 7.19 \\
\hline Day 5 & 7.38 & 6.95 & 6.93 & 6.89 & 6.84 & 6.71 \\
\hline Day 6 & 6.78 & 7.93 & 8.52 & 8.48 & 8.30 & 8.19 \\
\hline Final & 7.31 & 7.26 & 7.15 & 7.13 & 7.09 & 7.14 \\
\hline
\end{tabular}

Dissolved Oxygen (Before Renewal)

\begin{tabular}{|l|r|r|r|r|r|r|}
\hline & Tentrol & $6.25 \%$ & $12.5 \%$ & $25 \%$ & $50 \%$ & $100 \%$ \\
\hline Day 1 & 6.8 & 7.6 & 7.6 & 7.4 & 7.4 & 7.2 \\
\hline Day 2 & 7.9 & 8.2 & 8.3 & 8.3 & 8.4 & 8.6 \\
\hline Day 3 & 8.1 & 8.3 & 8.3 & 8.3 & 8.3 & 8.3 \\
\hline Day 4 & 8.1 & 8.6 & 8.6 & 8.6 & 8.6 & 8.0 \\
\hline Day 5 & 8.2 & 8.6 & 8.6 & 8.6 & 8.7 & 8.7 \\
\hline Day 6 & 8.4 & 8.5 & 8.5 & 8.5 & 8.4 & 8.4 \\
\hline Final & 8.1 & 8.4 & 8.4 & 8.4 & 8.3 & 8.3 \\
\hline
\end{tabular}

Test Results Reviewed and Approved By:

Temperature
\begin{tabular}{|l|r|}
\hline \multicolumn{1}{|c|}{ Incubator ${ }^{\circ} \mathrm{C}$} \\
\hline Initial & 25.1 \\
\hline Day 1 & 24.3 \\
\hline Day 2 & 24.5 \\
\hline Day 3 & 24.4 \\
\hline Day 4 & 24.5 \\
\hline Day 5 & 24.2 \\
\hline Day 6 & 24.4 \\
\hline Final & 24.5 \\
\hline
\end{tabular}




\title{
7 Day Chronic Definitive Survival and Reproduction Bioassay \\ Method: EPA/600/4-91/002
}

\author{
Test Organism:
}

Daphnia ambigua

Facility: WSRC
Sample ID: G-10

Aiken County

ETTH: 19987

Sample ID: June 4, 2002 


\section{CHRONIC DEFINITIVE SURVIVAL AND REPRODUCTION TEST Survival and Reproduction Results}

Client: WSRC
Sample Identification:
Test Date:
\begin{tabular}{|c|c|c|c|c|c|c|}
\hline Reproduction at \\
\hline Rep. & Day 3/4 & Day 5 & Day 6 & Day 7 & Day 8 & Total \\
\hline 1 & 0 & 6 & 8 & 0 & 12 & 26 \\
\hline 2 & 0 & 6 & 10 & 0 & 8 & 24 \\
\hline 3 & 0 & 4 & 10 & 0 & 14 & 28 \\
\hline 4 & 0 & 5 & 12 & 0 & 6 & 23 \\
\hline 5 & 0 & 4 & 8 & $X$ & $X$ & 12 \\
\hline 6 & 0 & 5 & 13 & 0 & 8 & 26 \\
\hline 7 & 0 & 3 & 9 & 0 & 10 & 22 \\
\hline 8 & 0 & 3 & 11 & 0 & 6 & 20 \\
\hline 9 & 0 & 5 & 0 & 11 & 2 & 18 \\
\hline 10 & 0 & 6 & $X$ & $X$ & $X$ & 6 \\
\hline
\end{tabular}

County:

ETT \#:
Aiken

$\mathrm{SC}$

19987

\begin{tabular}{|c|c|c|c|c|c|c|}
\hline \multicolumn{2}{|c|}{ Reproduction at } & \multicolumn{2}{c|}{$6.25 \%$} & \multicolumn{2}{c|}{ Concentration } \\
\hline Rep. & Day 3/4 & Day 5 & Day 6 & Day 7 & Day 8 & Total \\
\hline 1 & 0 & 5 & 12 & 0 & 13 & 30 \\
\hline 2 & 0 & 4 & 11 & 0 & 6 & 21 \\
\hline 3 & 3 & 0 & 10 & 11 & 0 & 24 \\
\hline 4 & 0 & 6 & 12 & $\times$ & $\times$ & 18 \\
\hline 5 & 0 & 5 & 13 & 0 & 9 & 27 \\
\hline 6 & 0 & 6 & 11 & 0 & 8 & 25 \\
\hline 7 & 0 & 7 & 10 & 0 & 9 & 26 \\
\hline 8 & 0 & 4 & 9 & 0 & 12 & 25 \\
\hline 9 & 0 & 4 & 11 & 0 & 12 & 27 \\
\hline 10 & 5 & 0 & 12 & 12 & 0 & 29 \\
\hline
\end{tabular}

\begin{tabular}{|c|c|c|c|c|c|c|}
\hline \multicolumn{1}{|c|}{ Reproduction at } & \multicolumn{3}{c|}{$12.5 \%$} & \multicolumn{1}{c|}{ Concentration } \\
\hline Rep. & Day 3/4 & Day 5 & Day 6 & Day 7 & Day 8 & Total \\
\hline 1 & 4 & 0 & 12 & 12 & 0 & 28 \\
\hline 2 & 0 & 4 & 15 & 0 & 2 & 21 \\
\hline 3 & 4 & 0 & 14 & 13 & 0 & 31 \\
\hline 4 & 0 & 6 & 13 & 0 & 11 & 30 \\
\hline 5 & 0 & 3 & 12 & 0 & 10 & 25 \\
\hline 6 & 0 & 4 & 13 & 0 & 12 & 29 \\
\hline 7 & 0 & 6 & 12 & 0 & 14 & 32 \\
\hline 8 & 0 & 2 & 10 & 0 & 4 & 16 \\
\hline 9 & 0 & 4 & 9 & 0 & 0 & 13 \\
\hline 10 & 0 & 5 & 11 & 0 & 3 & 19 \\
\hline
\end{tabular}

\begin{tabular}{|c|c|c|c|c|c|c|}
\hline \multicolumn{2}{|c|}{ Reproduction at } & \multicolumn{2}{c|}{$25 \%$} & \multicolumn{3}{c|}{ Concentration } \\
\hline Rep. & Day 3/4 & Day 5 & Day 6 & Day 7 & Day 8 & Total \\
\hline 1 & 0 & 5 & 9 & 12 & 0 & 26 \\
\hline 2 & 0 & 6 & 11 & 0 & 9 & 26 \\
\hline 3 & 5 & 0 & 12 & 10 & 0 & 27 \\
\hline 4 & 0 & 2 & 12 & 0 & 7 & 21 \\
\hline 5 & 0 & 5 & 10 & $X$ & $X$ & 15 \\
\hline 6 & 0 & 4 & 9 & 0 & 8 & 21 \\
\hline 7 & 0 & 3 & 12 & 0 & 11 & 26 \\
\hline 8 & 0 & 6 & 11 & 0 & 14 & 31 \\
\hline 9 & 0 & 4 & 11 & 0 & 11 & 26 \\
\hline 10 & male & $L$ & $L$ & $L$ & $L$ & $L$ \\
\hline
\end{tabular}

\begin{tabular}{|c|c|c|c|c|c|c|}
\hline Reproduction at & \multicolumn{3}{c|}{$50 \%$} & \multicolumn{3}{c|}{ Concentration } \\
\hline Rep. & Day 3/4 & Day 5 & Day 6 & Day 7 & Day & Toul \\
\hline 1 & 0 & 6 & 9 & 0 & 13 & 28 \\
\hline 2 & 4 & 0 & 7 & 13 & 0 & 24 \\
\hline 3 & 3 & 0 & 8 & 12 & 0 & 23 \\
\hline 4 & 0 & 5 & 8 & 0 & 5 & 18 \\
\hline 5 & 0 & 4 & 5 & 0 & 11 & 20 \\
\hline 6 & 0 & 5 & 11 & 0 & 10 & 26 \\
\hline 7 & 0 & 0 & 12 & 0 & 0 & 21 \\
\hline 8 & 0 & 3 & 11 & 0 & 7 & 21 \\
\hline 9 & 0 & 6 & 12 & 0 & 11 & 29 \\
\hline 10 & 4 & 0 & 10 & 10 & 0 & 24 \\
\hline
\end{tabular}

\begin{tabular}{|c|c|c|c|c|c|c}
\hline \multicolumn{1}{|c|}{ Reproduction at } & \multicolumn{2}{c}{$100 \%$} & \multicolumn{1}{c}{ Concentration } \\
\hline Rep. & Day 314 & Day 5 & Day 6 & Day 7 & Day 8 & Total \\
\hline 1 & $X$ & $X$ & $X$ & $X$ & $X$ & 0 \\
\hline 2 & 0 & 4 & 9 & 0 & 10 & 23 \\
\hline 3 & 3 & 0 & 9 & 12 & 0 & 24 \\
\hline 4 & 0 & 3 & 8 & 0 & 10 & 21 \\
\hline 5 & 0 & 2 & 10 & 0 & 12 & 24 \\
\hline 6 & 0 & 6 & 11 & 0 & 10 & 27 \\
\hline 7 & 0 & 5 & 7 & 0 & 13 & 25 \\
\hline 8 & $X$ & $X$ & $X$ & $X$ & $X$ & 0 \\
\hline 9 & 0 & 5 & 8 & 0 & 10 & 23 \\
\hline 10 & male & $L$ & $L$ & $L$ & $L$ & $L$ \\
\hline
\end{tabular}




\section{CHRONIC DEFINITIVE SURVIVAL AND REPRODUCTION TEST \\ Statistical Analyses}

Client: $\quad$ WSRC

Sample Identification: $\quad$ G-10

Test Date:

June 4, 2002

\begin{tabular}{|lll}
\hline Test for Normality & & \\
& & \\
Test Used: Kolmogorov D & critical & NA
\end{tabular}

NA
Test for Homogeneity of Variance

Test Used: Bartlett's Test

$$
B=N A
$$

critical NA
NA

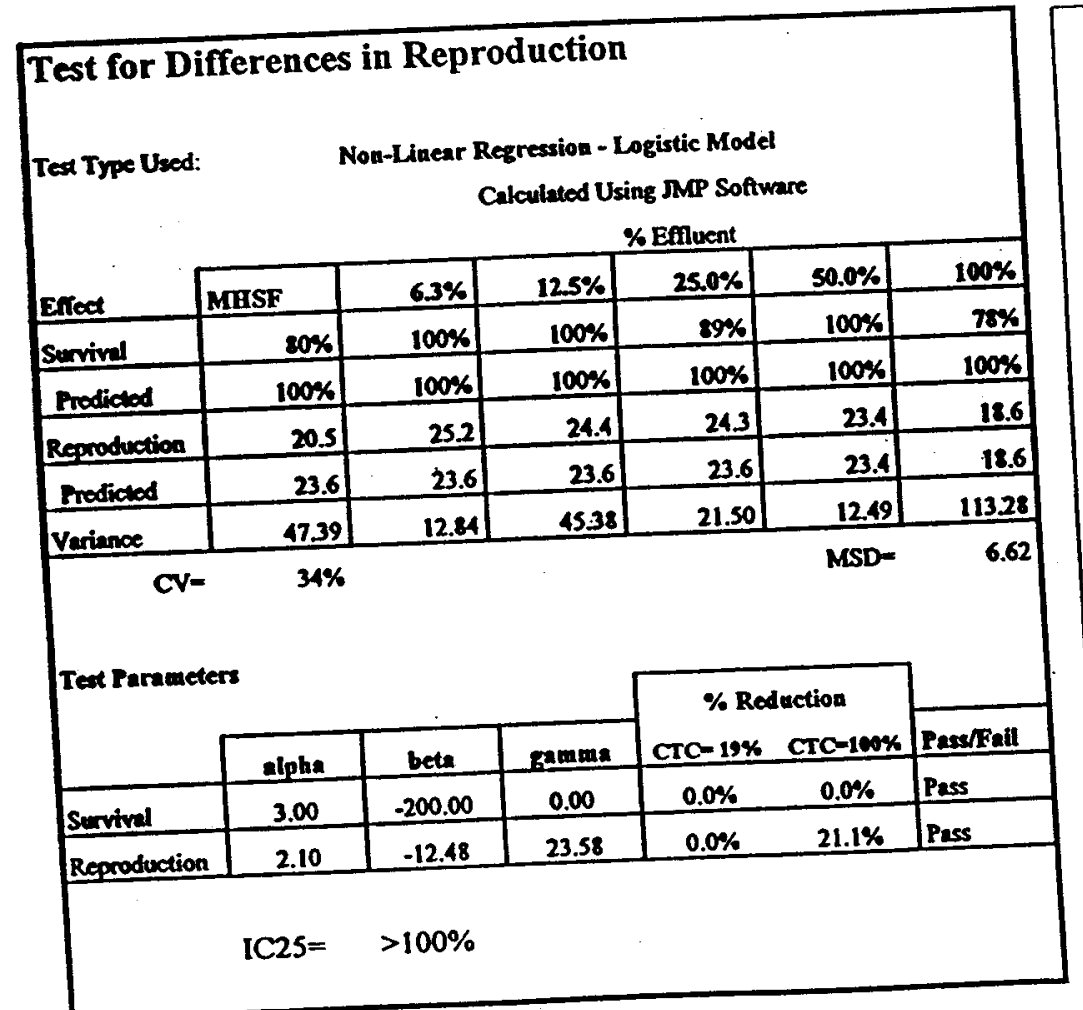

Summary

\section{Reproduction vs. Effluent Conc.}

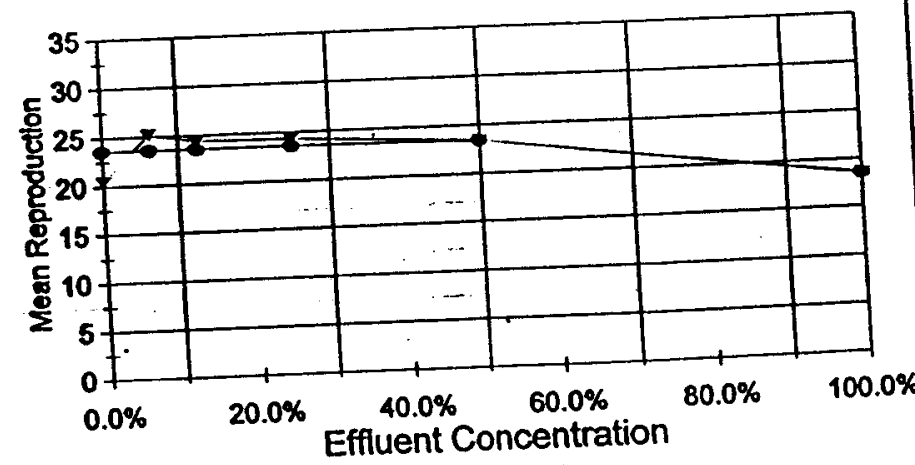

$\rightarrow$ Actual Reproduction $\ldots$ Predicted Reproduction 


\section{CHRONIC DEFINITIVE SURVIVAL AND REPRODUCTION TEST \\ Water Chemistry}

Client: WSRC

Sample Identification:

Test Date:

G-10

June 4, 2002

pH (After Renewal)

\begin{tabular}{|l|r|r|r|r|r|r|}
\hline \multicolumn{7}{|c|}{ Test Concentration } \\
\hline & control & $6.25 \%$ & $12.5 \%$ & $25 \%$ & $50 \%$ & $100 \%$ \\
\hline Initial & 7.21 & 7.05 & 7.06 & 7.01 & 7.06 & 7.06 \\
\hline Day 1 & 6.82 & 6.83 & 6.85 & 6.91 & 7.03 & 7.15 \\
\hline Day 2 & 6.92 & 7.19 & 6.97 & 6.90 & 6.98 & 6.99 \\
\hline Day 3 & 7.40 & 7.15 & 7.16 & 7.04 & 7.04 & 7.15 \\
\hline Day 4 & 7.00 & 7.21 & 7.03 & 7.00 & 6.98 & 7.00 \\
\hline Day 5 & 7.33 & 7.19 & 7.17 & 7.09 & 7.03 & 7.00 \\
\hline Day 6 & 6.96 & 7.40 & 7.36 & 7.31 & 7.26 & 7.22 \\
\hline
\end{tabular}

Dissolved Oxygen (After Renewal)

\begin{tabular}{|c|c|c|c|c|c|c|}
\hline & \multicolumn{6}{|c|}{ Test Concentration } \\
\hline & control & $6.25 \%$ & $12.5 \%$ & $25 \%$ & $50 \%$ & $100 \%$ \\
\hline Initial & 8.1 & 8.1 & 8.1 & 8.1 & 8.1 & 8.7 \\
\hline Day 1 & 8.0 & 8.3 & 8.2 & 8.2 & 8.0 & 7.8 \\
\hline Day 2 & 8.1 & 8.1 & 8.1 & 8.1 & 8.1 & 8.1 \\
\hline Day 3 & 8.2 & 8.3 & 8.3 & 8.2 & 8.2 & 8.2 \\
\hline Day 4 & 8.3 & 8.3 & 8.3 & 8.3 & 8.3 & 8.2 \\
\hline Day 5 & 8.4 & 8.3 & 8.3 & 8.3 & 8.2 & 8.2 \\
\hline Day 6 & 8.4 & 8.3 & 8.3 & 8.2 & 8.2 & 8.1 \\
\hline
\end{tabular}

$\mathrm{pH}$ (Before Renewal)

\begin{tabular}{|l|r|r|r|r|r|r|}
\hline & \multicolumn{7}{|c|}{ Test Concentration } \\
\hline Day 1 & 6.82 & 6.93 & 6.90 & 6.95 & 7.06 & 7.17 \\
\hline Day 2 & 7.15 & 7.37 & 7.21 & 7.13 & 7.13 & 7.19 \\
\hline Day 3 & 7.45 & 7.11 & 7.03 & 7.03 & 6.99 & 7.11 \\
\hline Day 4 & 7.20 & 7.40 & 7.39 & 7.33 & 7.30 & 7.18 \\
\hline Day 5 & 7.38 & 7.20 & 7.19 & 7.15 & 7.06 & 7.04 \\
\hline Day 6 & 6.78 & 7.88 & 7.89 & 7.72 & 7.62 & 7.62 \\
\hline Final & 7.31 & 7.03 & 7.06 & 7.13 & 7.35 & 7.44 \\
\hline
\end{tabular}

Dissolved Oxygen (Before Renewal)

\begin{tabular}{|l|r|r|r|r|r|r|}
\hline & \multicolumn{7}{|c|}{ Test Concentration } \\
\hline & control & $6.25 \%$ & $12.5 \%$ & $25 \%$ & $50 \%$ & $100 \%$ \\
\hline Day 1 & 6.8 & 7.9 & 7.8 & 7.6 & 7.4 & 7.2 \\
\hline Day 2 & 7.9 & 8.0 & 8.0 & 8.0 & 7.9 & 7.5 \\
\hline Day 3 & 8.1 & 8.3 & 8.2 & 8.2 & 8.1 & 8.1 \\
\hline Day 4 & 8.1 & 8.3 & 8.3 & 8.3 & 8.2 & 8.2 \\
\hline Day 5 & 8.2 & 8.3 & 8.3 & 8.3 & 8.2 & 8.1 \\
\hline Day 6 & 8.4 & 8.6 & 8.6 & 8.6 & 8.5 & 8.1 \\
\hline Final & 8.1 & 7.1 & 7.1 & 7.1 & 7.2 & 7. \\
\hline
\end{tabular}

Temperature

Temperature
\begin{tabular}{|l|r|}
\hline Incubator ${ }^{\circ} \mathrm{C}$ \\
\hline Initial & 25.1 \\
\hline Day 1 & 24.3 \\
\hline Day 2 & 24.5 \\
\hline Day 3 & 24.4 \\
\hline Day 4 & 24.5 \\
\hline Day 5 & 24.2 \\
\hline Day 6 & 24.4 \\
\hline Final & 24.5 \\
\hline
\end{tabular}

Test Results Reviewed and Approved By: 


\title{
7 Day Chronic Definitive Survival and Reproduction Bioassay Method: EPA/600/4-91/002
}

Test Organism:

Daphnia ambigua

\author{
Facility: WSRC \\ Sample ID: A-01
}

Aiken County

ETT\#: 20212

Sample ID: July 16, 2002 


\section{CHRONIC DEFINITIVE SURVIVAL AND REPRODUCTION TEST \\ Survival and Reproduction Results}

\begin{tabular}{|c|c|c|c|}
\hline & & County: & Aiken \\
\hline Client: & WSRC & NPDES\#: & $\mathrm{sC}$ \\
\hline Sample Identification: & $\begin{array}{r}A-01 \\
\text { July } 16,2002\end{array}$ & ETT \#: & 20212 \\
\hline
\end{tabular}

\begin{tabular}{|c|c|c|c|c|c|c|}
\hline Reproduction at & \multicolumn{7}{c|}{ MuSF } & Control \\
\hline Rep. & Day $3 / 4$ & Day 5 & Day 6 & Day 7 & Day 8 & Total \\
\hline 1 & 3 & 0 & 9 & 16 & 0 & 28 \\
\hline 2 & 4 & 0 & 13 & 0 & 12 & 29 \\
\hline 3 & 0 & 5 & 10 & 0 & 11 & 26 \\
\hline 4 & 5 & 0 & 9 & 12 & 0 & 26 \\
\hline 5 & 0 & 5 & 9 & 0 & 11 & 25 \\
\hline 6 & 4 & 0 & 8 & 4 & 7 & 23 \\
\hline 7 & 4 & 0 & 8 & 0 & 12 & 24 \\
\hline 8 & 4 & 0 & 4 & 13 & 0 & 21 \\
\hline 9 & 0 & 0 & 9 & 0 & 12 & 21 \\
\hline 10 & 0 & 2 & 8 & 0 & 14 & 24 \\
\hline
\end{tabular}

\begin{tabular}{|c|c|c|c|c|c|c|}
\hline Reproduction at & \multicolumn{9}{c|}{$6.25 \%$} & \multicolumn{2}{c|}{ Concentration } \\
\hline Rep. & Day 3/4 & Day 5 & Day 6 & Day 7 & Day 8 & Total \\
\hline 1 & 4 & 0 & 11 & 14 & 0 & 29 \\
\hline 2 & 3 & 0 & 9 & 15 & 0 & 27 \\
\hline 3 & 0 & 6 & 9 & 0 & 12 & 27 \\
\hline 4 & 2 & 0 & 7 & 13 & 0 & 22 \\
\hline 5 & 0 & 5 & 9 & 0 & 11 & 25 \\
\hline 6 & 4 & 1 & 9 & 14 & 0 & 28 \\
\hline 7 & 4 & 0 & 10 & 9 & 0 & 23 \\
\hline 8 & 5 & 0 & 11 & 14 & 0 & 30 \\
\hline 9 & 0 & 5 & 12 & 0 & 14 & 31 \\
\hline 10 & 0 & 5 & 13 & 0 & 13 & 31 \\
\hline
\end{tabular}

\begin{tabular}{|c|c|c|c|c|c|c|}
\hline Reproduction at & \multicolumn{3}{|c|}{$12.5 \%$} & \multicolumn{3}{c|}{ Concentration } \\
\hline Rep. & Day 3/4 & Day 5 & Day 6 & Day 7 & Day 8 & Total \\
\hline 1 & 4 & 0 & 12 & 13 & 0 & 29 \\
\hline 2 & 5 & 0 & 8 & 15 & 0 & 28 \\
\hline 3 & 0 & 5 & 14 & 0 & 10 & 29 \\
\hline 4 & 5 & 0 & 12 & 10 & 0 & 27 \\
\hline 5 & 3 & 0 & 14 & 14 & 0 & 31 \\
\hline 6 & 4 & 0 & 7 & 14 & 0 & 25 \\
\hline 7 & 3 & 0 & 9 & 15 & 0 & 27 \\
\hline 8 & 4 & 0 & $X$ & $X$ & $X$ & 4 \\
\hline 9 & 0 & 5 & 12 & 0 & 14 & 31 \\
\hline 10 & 0 & 6 & 10 & 12 & 0 & 28 \\
\hline
\end{tabular}

\begin{tabular}{|c|c|c|c|c|c|c|}
\hline \multicolumn{1}{|c|}{ Reproduction at } & \multicolumn{3}{c|}{$25 \%$} & \multicolumn{3}{c|}{ Concentration } \\
\hline Rep. & Day 3/4 & Day 5 & Day 6 & Day 7 & Day 8 & Toall \\
\hline 1 & 4 & 0 & 8 & 16 & 0 & 28 \\
\hline 2 & 5 & 0 & 12 & 16 & 0 & 33 \\
\hline 3 & 0 & 5 & 10 & 0 & 14 & 29 \\
\hline 4 & 7 & 0 & 8 & $X$ & $X$ & 15 \\
\hline 5 & 0 & 3 & 10 & 0 & 13 & 26 \\
\hline 6 & 5 & 0 & 12 & 15 & 0 & 32 \\
\hline 7 & 3 & 0 & 9 & 16 & 0 & 28 \\
\hline 8 & 7 & 0 & 10 & 14 & 0 & 31 \\
\hline 9 & 0 & 5 & 11 & 0 & 11 & 27 \\
\hline 10 & 0 & 4 & 12 & 0 & 13 & 29 \\
\hline
\end{tabular}

\begin{tabular}{|c|c|c|c|c|c|c|}
\hline \multicolumn{1}{|c|}{ Reproduction at } & \multicolumn{3}{|c|}{$50 \%$} & \multicolumn{3}{c|}{ Concentration } \\
\hline Rep. & Day $3 / 4$ & Day 5 & Day 6 & Day 7 & Day 8 & Toal \\
\hline 1 & 4 & 0 & 9 & 15 & 0 & 28 \\
\hline 2 & 4 & 0 & 11 & 17 & 0 & 32 \\
\hline 3 & 0 & 5 & 10 & 11 & 0 & 26 \\
\hline 4 & 3 & 0 & 13 & 14 & 0 & 30 \\
\hline 5 & 0 & 5 & 10 & 0 & 15 & 30 \\
\hline 6 & 4 & 0 & 12 & 14 & 0 & 30 \\
\hline 7 & 4 & 0 & 11 & 16 & 0 & 31 \\
\hline 8 & 4 & 0 & 8 & 14 & 0 & 26 \\
\hline 9 & 0 & 7 & 11 & 0 & 15 & 33 \\
\hline 10 & 0 & 4 & 8 & 0 & 14 & 26 \\
\hline
\end{tabular}

\begin{tabular}{|c|c|c|c|c|c|c|}
\hline \multicolumn{9}{|c|}{ Reproduction at } & \multicolumn{9}{c|}{$100 \%$} & \multicolumn{2}{c|}{ Concentration } \\
\hline Rep. & Day $3 / 4$ & Day 5 & Day 6 & Day 7 & Dey 8 & Total \\
\hline 1 & 4 & 0 & 12 & 16 & 0 & 32 \\
\hline 2 & 4 & 0 & 11 & 13 & 0 & 28 \\
\hline 3 & 0 & 4 & 9 & 14 & 0 & 27 \\
\hline 4 & 0 & 7 & 14 & 15 & 0 & 36 \\
\hline 5 & 4 & 0 & 13 & 15 & 0 & 32 \\
\hline 6 & 4 & 0 & 14 & 16 & 0 & 34 \\
\hline 7 & 0 & 5 & 12 & 0 & 13 & 30 \\
\hline 8 & 0 & 3 & 9 & 14 & 0 & 26 \\
\hline 9 & 0 & 5 & 13 & 0 & 15 & 33 \\
\hline 10 & 0 & 6 & 12 & 0 & 14 & 32 \\
\hline
\end{tabular}




\section{CHRONIC DEFINITIVE SURVIVAL AND REPRODUCTION TEST \\ Statistical Analyses}

Client: $\quad$ WSRC

Sample Identification: A-01

Test Date: July 16, 2002

\begin{tabular}{|llll}
\hline Test for Normality & & \\
& & \\
Test Used: Kolmogorov D & $D=$ & NA \\
& critical & NA
\end{tabular}

Test for Homogeneity of Variance

Test Used: Bartlett's Test $\quad B=N A$

critical NA

NA

NA

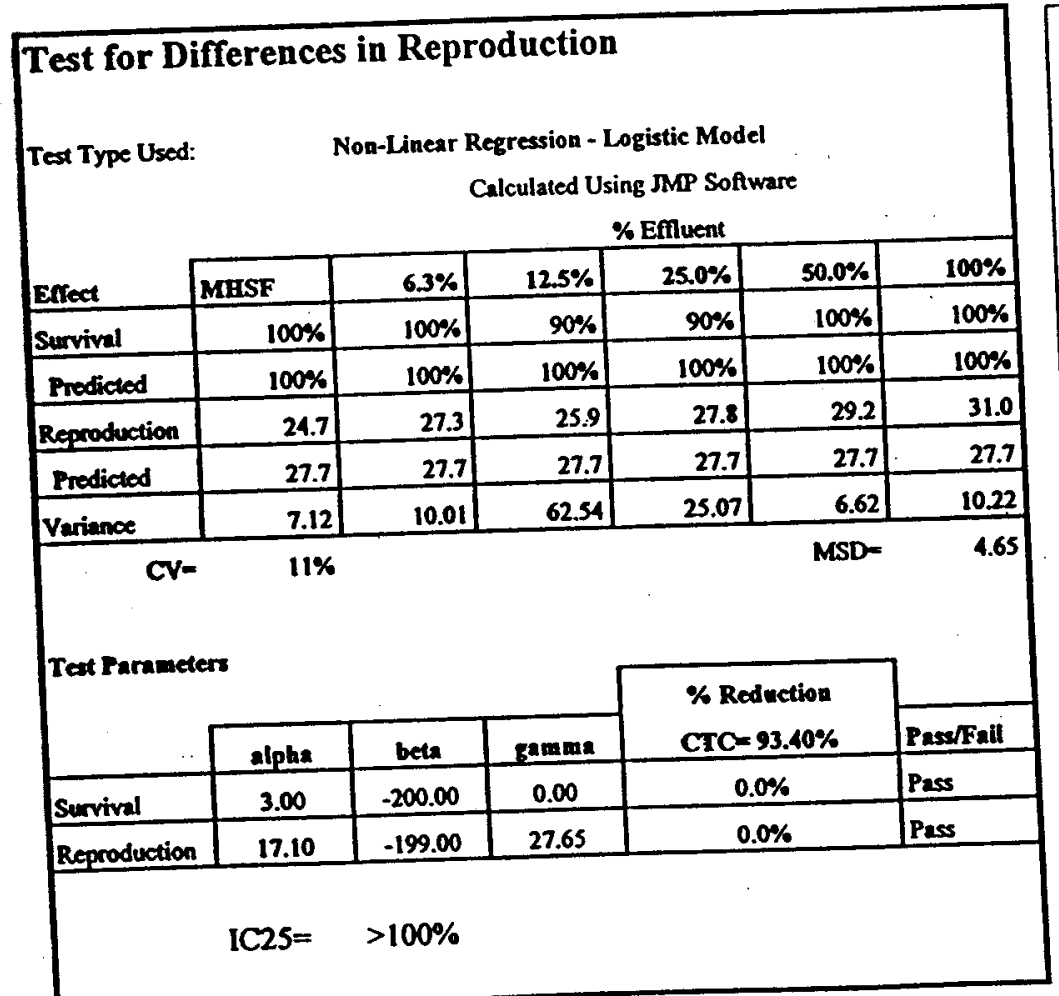

Reproduction vs. Effluent Conc.

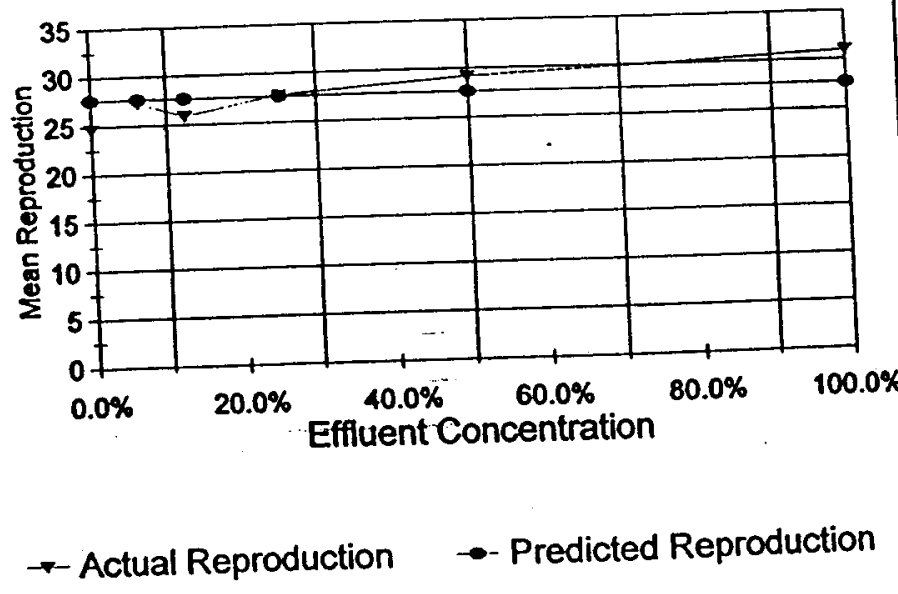

Summary

The reductions in survival and reproduction at the CTC are $<25 \%$ - Pass 


\section{Day Chronic Definitive Survival and Reproduction Bioassay Method: EPA/600/4-91/002}

Test Organism:

Ceriodaphnia dubia

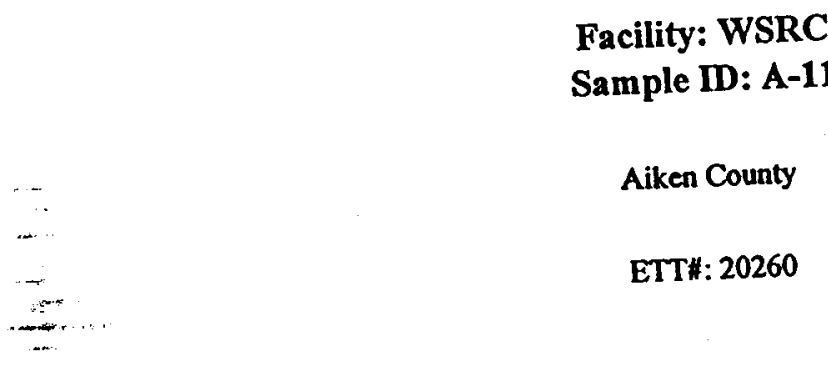

Sample ID: July 23, 2002 


\section{CHRONIC DEFINITIVE SURVIVAL AND REPRODUCTION TEST \\ Survival and Reproduction Results}

Client:
Sample Identification:
Test Date:
\begin{tabular}{|c|c|c|c|c|c|c|}
\hline Reproduction at & A-11 \\
\hline Rep. & Day 3/4 & Day 5 & Day 6 & Day 7 & Day 8 & Total \\
\hline 1 & 0 & 3 & 9 & 0 & 10 & 22 \\
\hline 2 & 0 & 2 & 6 & 0 & 9 & 17 \\
\hline 3 & 0 & 3 & 7 & 0 & 10 & 20 \\
\hline 4 & 0 & 0 & 6 & 10 & 0 & 16 \\
\hline 5 & 0 & 5 & 0 & 7 & 12 & 24 \\
\hline 6 & 0 & 2 & $X$ & $X$ & $X$ & 2 \\
\hline 7 & 0 & 4 & 8 & 9 & 0 & 21 \\
\hline 8 & 4 & 0 & 7 & 9 & 0 & 20 \\
\hline 9 & 5 & 0 & 6 & 10 & 0 & 21 \\
\hline 10 & 0 & 6 & 10 & 1 & 13 & 30 \\
\hline
\end{tabular}

$\begin{array}{ll}\text { County: } & \text { Aiken } \\ \text { NPDES\#: } & \text { SC } \\ \text { ETT \#: } & 20260\end{array}$

\begin{tabular}{|c|c|c|c|c|c|c|}
\hline \multicolumn{2}{|c|}{ Reproduction at } & \multicolumn{2}{c|}{$6.25 \%$} & \multicolumn{3}{c|}{ Concentration } \\
\hline Rep. & Day 3/4 & Day 5 & Day 6 & Day 7 & Day 8 & Total \\
\hline 1 & 0 & 4 & 14 & 0 & 13 & 31 \\
\hline 2 & 0 & 3 & 8 & 0 & 14 & 25 \\
\hline 3 & 0 & 3 & 8 & 11 & 0 & 22 \\
\hline 4 & 3 & 0 & 8 & 13 & 0 & 24 \\
\hline 5 & 0 & 3 & $X$ & $X$ & $X$ & 3 \\
\hline 6 & 0 & 5 & 7 & 0 & 9 & 21 \\
\hline 7 & 4 & 0 & 9 & 9 & 0 & 22 \\
\hline 8 & 0 & 4 & 5 & 0 & 10 & 19 \\
\hline 9 & 0 & 4 & 9 & 0 & 10 & 23 \\
\hline 10 & 0 & 2 & 7 & 0 & 8 & 17 \\
\hline
\end{tabular}

\begin{tabular}{|c|c|c|c|c|c|c|}
\hline Reproduction at & \multicolumn{4}{c|}{$12.5 \%$} & \multicolumn{1}{c|}{ Concentration } \\
\hline Rep. & Day 3/4 & Day 5 & Day 6 & Day 7 & Day & Total \\
\hline 1 & 0 & 5 & 11 & 0 & 13 & 29 \\
\hline 2 & 0 & 3 & 0 & 14 & 0 & 17 \\
\hline 3 & 3 & 0 & 9 & 10 & 0 & 22 \\
\hline 4 & 0 & 5 & 0 & 9 & 10 & 24 \\
\hline 5 & 0 & 3 & 5 & 0 & 9 & 17 \\
\hline 6 & 0 & 4 & $X$ & $X$ & $X$ & 4 \\
\hline 7 & 4 & 0 & 7 & 12 & 0 & 23 \\
\hline 8 & 0 & 6 & 10 & 0 & 15 & 31 \\
\hline 9 & 5 & 0 & 7 & 9 & 0 & 21 \\
\hline 10 & 0 & 5 & 8 & 0 & 12 & 25 \\
\hline
\end{tabular}

\begin{tabular}{|c|c|c|c|c|c|c|}
\hline \multicolumn{2}{|c|}{ Reproduction at } & \multicolumn{2}{c|}{$25 \%$} & \multicolumn{3}{c|}{ Concentration } \\
\hline Rep. & Day 3/4 & Day 5 & Day 6 & Day 7 & Day 8 & Total \\
\hline 1 & 0 & 1 & 7 & 0 & 12 & 20 \\
\hline 2 & 0 & 3 & 2 & 0 & 7 & 12 \\
\hline 3 & 0 & 4 & 7 & 0 & 12 & 23 \\
\hline 4 & 0 & $X$ & $X$ & $X$ & $X$ & 0 \\
\hline 5 & 0 & 2 & 6 & 7 & 0 & 15 \\
\hline 6 & $X$ & $X$ & $X$ & $X$ & $X$ & 0 \\
\hline 7 & 0 & 3 & 8 & 0 & 0 & 11 \\
\hline 8 & 0 & 0 & 4 & 10 & 13 & 27 \\
\hline 9 & 0 & 0 & 4 & $X$ & $X$ & 4 \\
\hline 10 & 0 & 4 & 0 & 12 & 13 & 29 \\
\hline
\end{tabular}

\begin{tabular}{|c|c|c|c|c|c|c|}
\hline Reproduction at & \multicolumn{3}{c|}{$50 \%$} & \multicolumn{3}{c|}{ Concentration } \\
\hline Rep. & Duy 3/4 & Day 5 & Day 6 & Day 7 & Day 8 & Total \\
\hline 1 & 3 & 0 & 7 & 15 & 0 & 25 \\
\hline 2 & 0 & 5 & 8 & 12 & 0 & 25 \\
\hline 3 & 0 & 4 & 7 & 0 & 11 & 22 \\
\hline 4 & 0 & 0 & 4 & 10 & 0 & 14 \\
\hline 5 & 0 & 3 & 5 & $X$ & $\times$ & 8 \\
\hline 6 & 0 & 4 & 0 & 9 & 0 & 13 \\
\hline 7 & 3 & 0 & 8 & 11 & 0 & 22 \\
\hline 8 & 0 & 3 & 5 & 0 & 11 & 19 \\
\hline 9 & 4 & 0 & 8 & 10 & 0 & 22 \\
\hline 10 & 0 & 5 & 0 & 12 & 13 & 30 \\
\hline
\end{tabular}

\begin{tabular}{|c|c|c|c|c|c|c|}
\hline Reproduction at & \multicolumn{4}{c|}{$100 \%$} & \multicolumn{2}{c|}{ Concentration } \\
\hline Rep. & Day 3/4 & Day 5 & Day 6 & Day 7 & Day & Toul \\
\hline 1 & 0 & 4 & 8 & 10 & 0 & 22 \\
\hline 2 & 0 & 2 & 5 & 0 & 10 & 17 \\
\hline 3 & 0 & 4 & 7 & 9 & 0 & 20 \\
\hline 4 & 0 & 0 & 8 & 0 & 14 & 22 \\
\hline 5 & 0 & 0 & 3 & 9 & 0 & 12 \\
\hline 6 & 0 & 3 & 8 & 0 & 10 & 21 \\
\hline 7 & 0 & 0 & 5 & 9 & 0 & 14 \\
\hline 8 & 0 & 3 & 0 & 8 & 0 & 11 \\
\hline 9 & 0 & 5 & 9 & 0 & 12 & 26 \\
\hline 10 & 0 & 4 & 10 & 7 & 0 & 21 \\
\hline
\end{tabular}




\title{
CHRONIC DEFINITIVE SURVIVAL AND REPRODUCTION TEST \\ Statistical Analyses
}

\begin{abstract}
Client: WSRC
Sample Identification: A-11

Test Date: July 23, 2002
\end{abstract}

\begin{tabular}{|lll}
\hline Test for Normality & & \\
Test Used: Kolmogorov.D & $\begin{array}{ll}\mathrm{D}= & \text { NA } \\
\text { critical } & \text { NA }\end{array}$
\end{tabular}

NA
Test for Homogeneity of Variance

Test Used: Bartlett's Test

$B=N A$

critical NA

NA

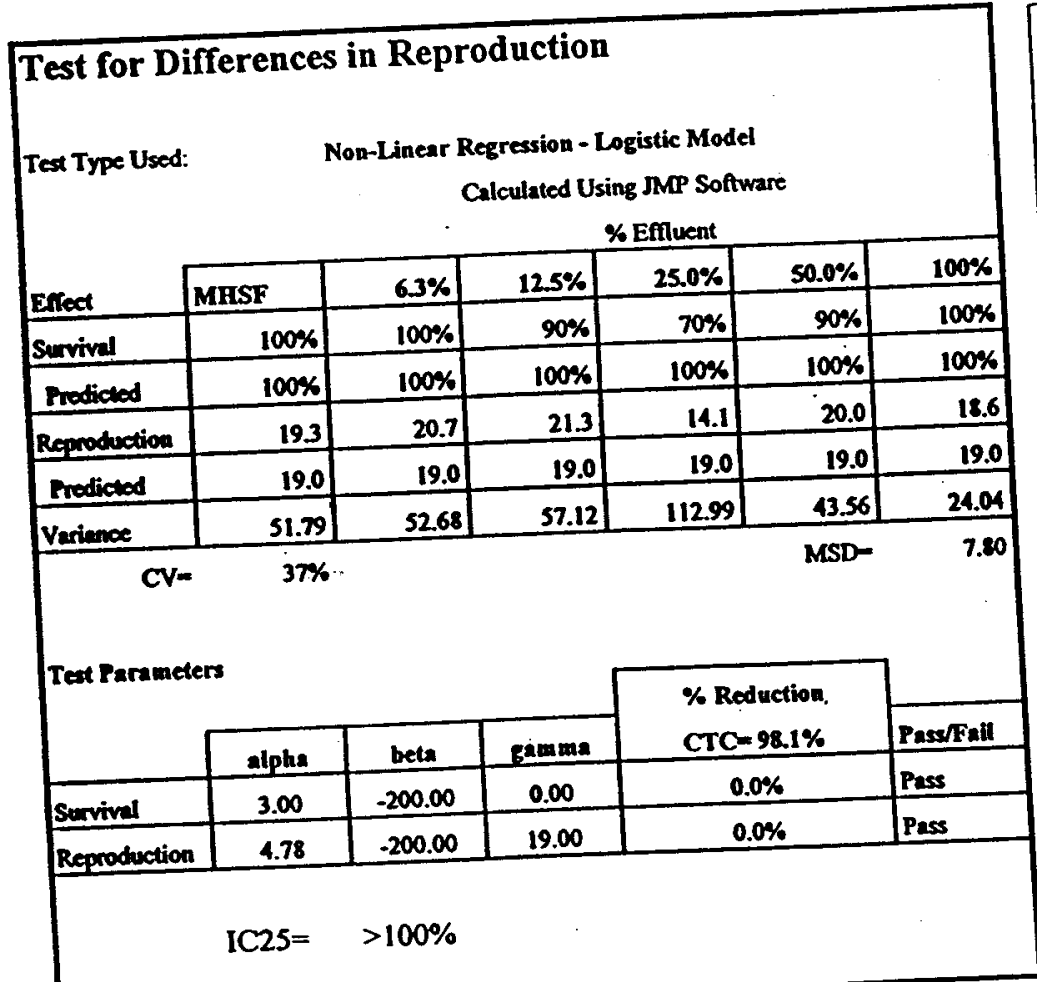

Reproduction vs. Effluent Conc.

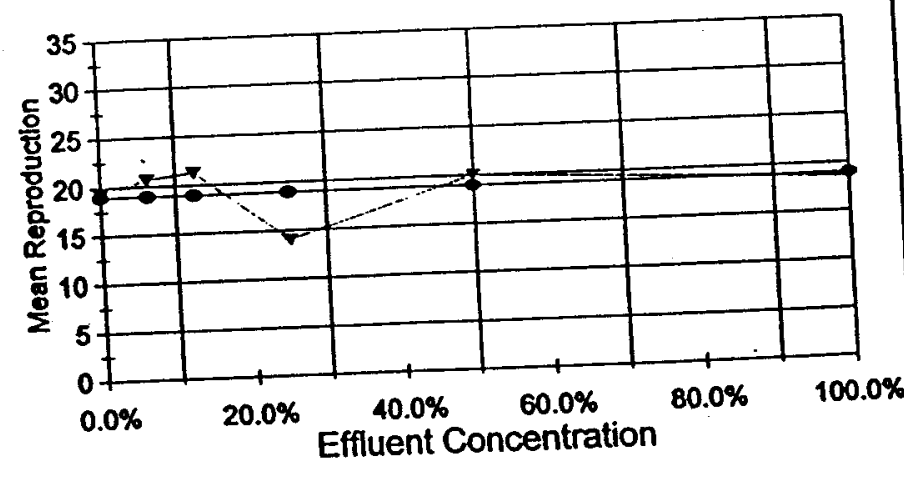

Actual Reproduction
- Predicted Reproduction

Summary
The reductions in survival and reproduction at the CTC are $<25 \%$ - Pass 


\section{Day Chronic Definitive Survival and Reproduction Bioassay \\ Method: EPA/600/4-91/002}

Test Organism:

Ceriodaphnia dubia

Facility: WSRC

Sample ID: G-10

Aiken County

ETT\#: 20242

Sample ID: July 18, 2002 


\section{CHRONIC DEFINITIVE SURVIVAL AND REPRODUCTION TEST Survival and Reproduction Results}

Client: WSRC
Sample Identification:
Test Date:
\begin{tabular}{|c|c|c|c|c|c|c|}
\hline Reproduction at 18,2002 \\
\hline Rep. & Day $3 / 4$ & Day 5 & Day 6 & Day 7 & Day 8 & Total \\
\hline 1 & 0 & 6 & 9 & 0 & 15 & 30 \\
\hline 2 & 0 & 5 & 5 & 0 & 10 & 20 \\
\hline 3 & 4 & 0 & 11 & 12 & 0 & 27 \\
\hline 4 & 5 & 0 & 9 & 12 & 0 & 26 \\
\hline 5 & 4 & 0 & 12 & 14 & 0 & 30 \\
\hline 6 & 0 & 6 & 11 & 0 & 13 & 30 \\
\hline 7 & 4 & 1 & 9 & 13 & 0 & 27 \\
\hline 8 & 0 & 5 & 10 & 0 & 14 & 29 \\
\hline 9 & 5 & 0 & 9 & 15 & 0 & 29 \\
\hline 10 & 0 & 6 & 11 & 0 & 5 & 22 \\
\hline
\end{tabular}

$\begin{array}{ll}\text { County: } & \text { Aiken } \\ \text { NPDESH: } & \text { SC } \\ \text { ETT \#: } & \mathbf{2 0 2 4 2}\end{array}$

\begin{tabular}{|c|c|c|c|c|c|c|}
\hline \multicolumn{2}{|c|}{ Reproduction at } & \multicolumn{2}{c|}{$6.25 \%$} & \multicolumn{2}{c|}{ Concentration } \\
\hline Rep. & Day 3/4 & Day 5 & Day 6 & Day 7 & Day 8 & Total \\
\hline 1 & 0 & 4 & 10 & 0 & 11 & 25 \\
\hline 2 & 0 & 6 & 12 & 0 & 13 & 31 \\
\hline 3 & 0 & 4 & 0 & 14 & 9 & 27 \\
\hline 4 & 4 & 0 & 10 & 10 & 0 & 24 \\
\hline 5 & $X$ & $X$ & $X$ & $X$ & $X$ & 0 \\
\hline 6 & 0 & 6 & 9 & 0 & 13 & 28 \\
\hline 7 & $X$ & $X$ & $X$ & $X$ & $X$ & 0 \\
\hline 8 & 0 & 6 & 11 & 0 & 13 & 30 \\
\hline 9 & 4 & 0 & 8 & 1 & 0 & 13 \\
\hline 10 & 0 & 6 & 10 & 0 & 10 & 26 \\
\hline
\end{tabular}

\begin{tabular}{|c|c|c|c|c|c|c|}
\hline \multicolumn{1}{|c|}{ Reproduction at } & \multicolumn{3}{c|}{$12.5 \%$} & \multicolumn{1}{c|}{ Concentration } \\
\hline Rep. & Day 3/4 & Day 5 & Day 6 & Day 7 & Day 8 & Toual \\
\hline 1 & 0 & 6 & 9 & 0 & 14 & 29 \\
\hline 2 & 0 & 3 & 9 & 0 & 11 & 23 \\
\hline 3 & 0 & 7 & 8 & 0 & 16 & 31 \\
\hline 4 & 3 & 0 & 8 & 14 & 0 & 25 \\
\hline 5 & 6 & 0 & 10 & 0 & 15 & 31 \\
\hline 6 & 0 & 6 & 12 & 0 & 15 & 33 \\
\hline 7 & 4 & 0 & 8 & 12 & 0 & 24 \\
\hline 8 & $X$ & $X$ & $X$ & $X$ & $X$ & 0 \\
\hline 9 & 4 & 0 & 9 & 13 & 0 & 26 \\
\hline 10 & 0 & 4 & 9 & 0 & 12 & 25 \\
\hline
\end{tabular}

\begin{tabular}{|c|c|c|c|c|c|c|}
\hline \multicolumn{1}{|c|}{ Reproduction at } & \multicolumn{2}{|c|}{$25 \%$} & \multicolumn{3}{c|}{ Concentration } \\
\hline Rep. & Day 3/4 & Day 5 & Day 6 & Day 7 & Day 8 & Total \\
\hline 1 & 0 & 0 & 13 & 0 & 13 & 26 \\
\hline 2 & 0 & 5 & 10 & 0 & 13 & 28 \\
\hline 3 & 4 & 0 & 10 & 12 & 0 & 26 \\
\hline 4 & 4 & 0 & 10 & 12 & 0 & 26 \\
\hline 5 & 0 & 6 & 9 & 0 & 14 & 29 \\
\hline 6 & 0 & 6 & 12 & 0 & 12 & 30 \\
\hline 7 & 5 & 1 & 12 & 9 & 0 & 27 \\
\hline 8 & 0 & 6 & 9 & 0 & 13 & 28 \\
\hline 9 & $X$ & $X$ & $X$ & $X$ & $X$ & 0 \\
\hline 10 & 0 & 5 & 10 & 0 & 10 & 25 \\
\hline
\end{tabular}

\begin{tabular}{|c|c|c|c|c|c|c|}
\hline \multicolumn{2}{|c|}{ Reproduction at } & \multicolumn{4}{c|}{$50 \%$} & Concentration \\
\hline Rep. & Day 3/4 & Day 5 & Day 6 & Day 7 & Day & Total \\
\hline 1 & 0 & 0 & 10 & 0 & 12 & 22 \\
\hline 2 & 0 & 5 & 10 & 0 & 14 & 29 \\
\hline 3 & 5 & 1 & 10 & 9 & 0 & 25 \\
\hline 4 & 4 & 1 & 9 & 12 & 0 & 26 \\
\hline 5 & 5 & 0 & 11 & 11 & 0 & 27 \\
\hline 6 & 0 & 5 & 12 & 0 & 14 & 31 \\
\hline 7 & 6 & 0 & 9 & 0 & 16 & 31 \\
\hline 8 & 0 & 6 & 12 & 0 & 5 & 23 \\
\hline 9 & 7 & 0 & 10 & 12 & 0 & 29 \\
\hline 10 & 0 & 0 & 12 & 0 & 14 & 26 \\
\hline
\end{tabular}

\begin{tabular}{|c|c|c|c|c|c|c|}
\hline Reproduction at & \multicolumn{4}{c|}{$100 \%$} & \multicolumn{1}{c|}{ Concentration } \\
\hline Rep. & Day 3/4 & Day 5 & Day 6 & Day 7 & Day 8 & Total \\
\hline 1 & 0 & 4 & 8 & 0 & 11 & 23 \\
\hline 2 & 0 & 5 & 9 & 0 & 10 & 24 \\
\hline 3 & 4 & 0 & 10 & 10 & 0 & 24 \\
\hline 4 & 4 & 0 & 8 & 10 & 0 & 22 \\
\hline 5 & 5 & 0 & 10 & 7 & 0 & 22 \\
\hline 6 & 0 & 4 & 12 & 0 & 13 & 29 \\
\hline 7 & 0 & 0 & 2 & 0 & 3 & 5 \\
\hline 8 & 0 & 6 & 8 & 0 & $\times$ & 14 \\
\hline 9 & 4 & 0 & 7 & 15 & 0 & 26 \\
\hline 10 & 0 & 5 & 9 & 0 & 12 & 26 \\
\hline
\end{tabular}




\section{CHRONIC DEFINITIVE SURVIVAL AND REPRODUCTION TEST \\ Statistical Analyses}

Client: $\quad$ WSRC

Sample Identification:

G-10

Test Date:

July 18, 2002

\begin{tabular}{|c|c|c|}
\hline \multicolumn{3}{|l|}{ Test for Normality } \\
\hline Test Used: Kolmogorov D & $\begin{array}{l}\mathrm{D}= \\
\text { critical }\end{array}$ & $\begin{array}{l}\text { NA } \\
\text { NA }\end{array}$ \\
\hline NA & & \\
\hline
\end{tabular}

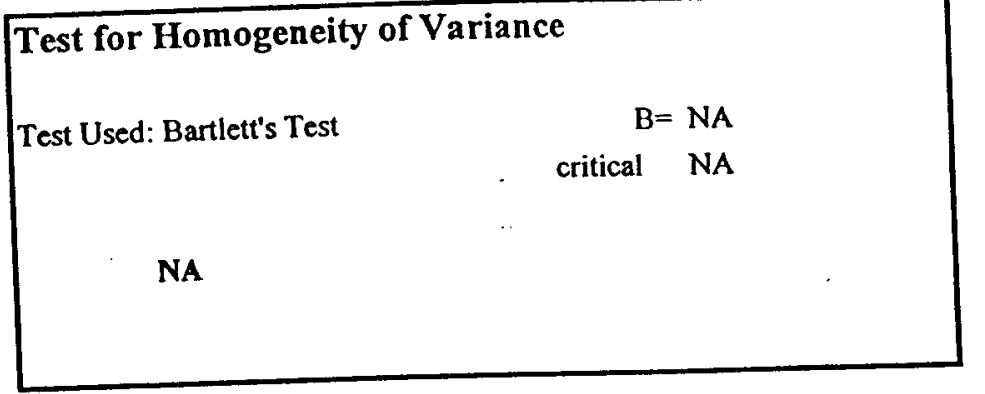

\begin{tabular}{|c|c|c|c|c|c|c|}
\hline \multicolumn{7}{|c|}{ Test for Differences in Reproduction } \\
\hline Test Type Used & \multicolumn{6}{|c|}{$\begin{array}{l}\text { Non-Linear Regression - Logistic Model } \\
\text { Calculated Using JMP Software } \\
\text { \%Effluent }\end{array}$} \\
\hline Errect & MHSF & $63 \%$ & $12.5 \%$ & $25.0 \%$ & $50.0 \%$ & $100 \%$ \\
\hline Servival & $100 \%$ & $100 \%$ & $90 \%$ & $90 \%$ & $100 \%$ & $90 \%$ \\
\hline Predicted & $100 \%$ & $100 \%$ & $100 \%$ & $100 \%$ & $100 \%$ & $100 \%$ \\
\hline Reproduction & 27.0 & 25.5 & 24.7 & 24.5 & 26.9 & 21.5 \\
\hline Prodicted & 24.2 & 24.2 & 24.2 & 24.2 & 24.2 & 24.2 \\
\hline Varience & 12.22 & 31.14 & 86.90 & 76.50 & 9.66 & 48.94 \\
\hline \multirow{2}{*}{\multicolumn{4}{|c|}{ Test Parameters }} & & MSD- & 6.90 \\
\hline & & & & \multirow{2}{*}{\multicolumn{2}{|c|}{$\begin{array}{l}\% \text { Reduction } \\
\text { CTC }=100 \%\end{array}$}} & \\
\hline & slpha & beta & gammar & & & Passuffail \\
\hline Survival & 3.00 & -200.00 & 0.00 & \multicolumn{2}{|c|}{$0.0 \%$} & Pass \\
\hline Reproduction & 17.10 & -199.00 & 24.20 & \multicolumn{2}{|c|}{$0.0 \%$} & Pass \\
\hline & IC25 $=$ & $>100 \%$ & & & & \\
\hline
\end{tabular}

Reproduction vs. Effluent Conc.

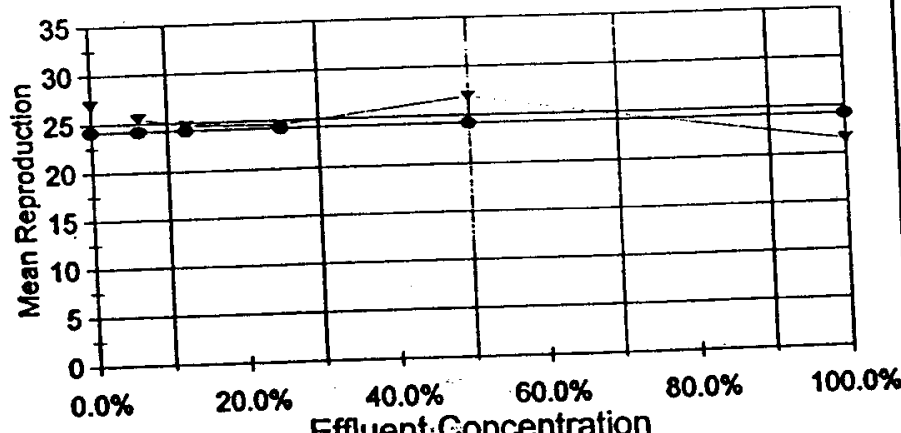

Effluent:Concentration

$\rightarrow$ Actual Reproduction $\quad \rightarrow$ Predicted Reproduction

\section{Summary}

The reductions in survival and reproduction at the CTC are $<25 \%$ - Pass 


\section{Day Chronic Definitive Survival and Reproduction Bioassay Method: EPA/600/4-91/002}

Test Organism: Daphnia ambigua

Facility: WSRC

Sample ID: A-01

Aiken County

ETT\#: 20333

Sample ID: August 6, 2002 


\section{CHRONIC DEFINITIVE SURVIVAL AND REPRODUCTION TEST Survival and Reproduction Results}

Client: $\quad$ WSRC

Sample Identification: A-01

Test Date:

August 6, 2002
County: Aiken

NPDESH: $\quad$ SC

ETT \#: $\quad 20333$

\begin{tabular}{|c|c|c|c|c|c|c|}
\hline \multicolumn{7}{|c|}{ Reproduction at } \\
\hline Rep. & Day 3/4 & Day 5 & Day 6 & Day 7 & Day 8 & Total \\
\hline 1 & 4 & 0 & 9 & 0 & 16 & 29 \\
\hline 2 & 0 & 5 & 11 & 0 & 15 & 31 \\
\hline 3 & 0 & 4 & 8 & 0 & 11 & 23 \\
\hline 4 & 0 & 4 & 9 & 0 & 14 & 27 \\
\hline 5 & 0 & 6 & 9 & 0 & 12 & 27 \\
\hline 6 & 0 & 5 & 10 & 0 & 13 & 28 \\
\hline 7 & 0 & 3 & 8 & 0 & 14 & 25 \\
\hline 8 & 0 & 4 & 10 & 0 & 12 & 26 \\
\hline 9 & 5 & 0 & 10 & $X$ & $X$ & 15 \\
\hline 10 & 2 & 4 & 12 & 0 & 0 & 18 \\
\hline
\end{tabular}

\begin{tabular}{|c|c|c|c|c|c|c|}
\hline \multicolumn{2}{|c|}{ Reproduction at } & \multicolumn{2}{c|}{$6.25 \%$} & \multicolumn{2}{c|}{ Concentration } \\
\hline Rep. & Day 3/4 & Day 5 & Day 6 & Day 7 & Day 8 & Total \\
\hline 1 & 3 & 0 & 8 & 0 & 13 & 24 \\
\hline 2 & 0 & 3 & 10 & 0 & 12 & 25 \\
\hline 3 & 0 & 3 & 7 & 0 & 13 & 23 \\
\hline 4 & 0 & 5 & 11 & 0 & 12 & 28 \\
\hline 5 & 0 & 4 & 12 & 0 & 13 & 29 \\
\hline 6 & 0 & 6 & 10 & 0 & 14 & 30 \\
\hline 7 & 0 & 4 & 9 & 0 & 12 & 25 \\
\hline 8 & 0 & 0 & 7 & 0 & 10 & 17 \\
\hline 9 & 5 & 0 & 13 & 13 & 0 & 31 \\
\hline 10 & 0 & 4 & 10 & 0 & 9 & 23 \\
\hline
\end{tabular}

\section{Reproduction at}

\begin{tabular}{|c|c|c|c|c|c|c|}
\hline Reproduction at & \multicolumn{4}{c|}{$12.5 \%$} & \multicolumn{3}{c|}{ Concentration } \\
\hline Rep. & Day 3/4 & Day 5 & Day 6 & Day 7 & Day 8 & Total \\
\hline 1 & 3 & 1 & 14 & 12 & 0 & 30 \\
\hline 2 & 0 & 4 & 7 & 0 & 10 & 21 \\
\hline 3 & 0 & 0 & 9 & 0 & 11 & 20 \\
\hline 4 & 0 & 6 & 8 & 0 & 13 & 27 \\
\hline 5 & 0 & 4 & 8 & 0 & 14 & 26 \\
\hline 6 & 0 & 5 & 10 & 0 & 14 & 29 \\
\hline 7 & 4 & 0 & 12 & 0 & 13 & 29 \\
\hline 8 & 3 & 0 & 10 & 0 & 12 & 25 \\
\hline 9 & 5 & 0 & 14 & 15 & 0 & 34 \\
\hline 10 & 0 & 6 & 11 & 0 & 14 & 31 \\
\hline
\end{tabular}

\begin{tabular}{|c|c|c|c|c|c|c|}
\hline Reproduction at & \multicolumn{4}{c|}{$50 \%$} & \multicolumn{3}{c|}{ Concentration } \\
\hline Rep. & Day 3/4 & Day 5 & Day 6 & Day 7 & Day 8 & Total \\
\hline 1 & 4 & 0 & 9 & 13 & 0 & 26 \\
\hline 2 & 0 & $X$ & $X$ & $X$ & $X$ & 0 \\
\hline 3 & 0 & 3 & 9 & 0 & 12 & 24 \\
\hline 4 & 0 & 5 & 10 & 0 & 14 & 29 \\
\hline 5 & 0 & 5 & 12 & 0 & 15 & 32 \\
\hline 6 & 0 & 6 & 11 & 0 & 14 & 31 \\
\hline 7 & 3 & 0 & 13 & 0 & 12 & 28 \\
\hline 8 & 0 & 0 & 8 & 0 & 10 & 18 \\
\hline 9 & 5 & 0 & 14 & 12 & 0 & 31 \\
\hline 10 & 0 & 4 & 10 & 0 & 13 & 27 \\
\hline
\end{tabular}

\begin{tabular}{|c|c|c|c|c|c|c|}
\hline Reproduction at & \multicolumn{4}{c|}{$25 \%$} & \multicolumn{1}{c|}{ Concentration } \\
\hline Rep. & Day 3/4 & Day 5 & Day 6 & Day 7 & Day 8 & Total \\
\hline 1 & 4 & 9 & 9 & 13 & 0 & 35 \\
\hline 2 & male & $L$ & $L$ & $L$ & $L$ & $L$ \\
\hline 3 & 0 & 5 & 8 & 0 & 11 & 24 \\
\hline 4 & 0 & 5 & 12 & 0 & 12 & 29 \\
\hline 5 & 0 & 6 & 9 & 0 & 13 & 28 \\
\hline 6 & 0 & 5 & 13 & 0 & 12 & 30 \\
\hline 7 & 0 & 3 & 10 & 0 & 14 & 27 \\
\hline 8 & 4 & 0 & 13 & 0 & 12 & 29 \\
\hline 9 & 6 & 0 & 14 & 13 & 0 & 33 \\
\hline 10 & 4 & 0 & 10 & 0 & 12 & 26 \\
\hline
\end{tabular}

\begin{tabular}{|c|c|c|c|c|c|c}
\hline \multicolumn{2}{|c}{ Reproduction at } & \multicolumn{2}{c}{$100 \%$} & \multicolumn{2}{c}{ Concentration } \\
\hline Rep. & Day 3/4 & Day 5 & Day 6 & Day 7 & Day 8 & Total \\
\hline 1 & 3 & 0 & 10 & 14 & 0 & 27 \\
\hline 2 & 0 & 0 & 8 & 13 & 0 & 21 \\
\hline 3 & 0 & 3 & 2 & 15 & 8 & 28 \\
\hline 4 & 0 & 4 & 14 & 0 & 13 & 31 \\
\hline 5 & 0 & 5 & 9 & 0 & 13 & 27 \\
\hline 6 & 0 & 6 & 12 & $X$ & $X$ & 18 \\
\hline 7 & 0 & 0 & 8 & 0 & 7 & 15 \\
\hline 8 & 4 & 0 & 10 & 12 & 0 & 26 \\
\hline 9 & 5 & 0 & 13 & 14 & 0 & 32 \\
\hline 10 & 3 & 0 & 12 & 0 & 15 & 30 \\
\hline
\end{tabular}




\section{CHRONIC DEFINITIVE SURVIVAL AND REPRODUCTION TEST \\ Statistical Analyses}

Client: WSRC

Sample Identification: A-01

Test Date:

August 6, 2002

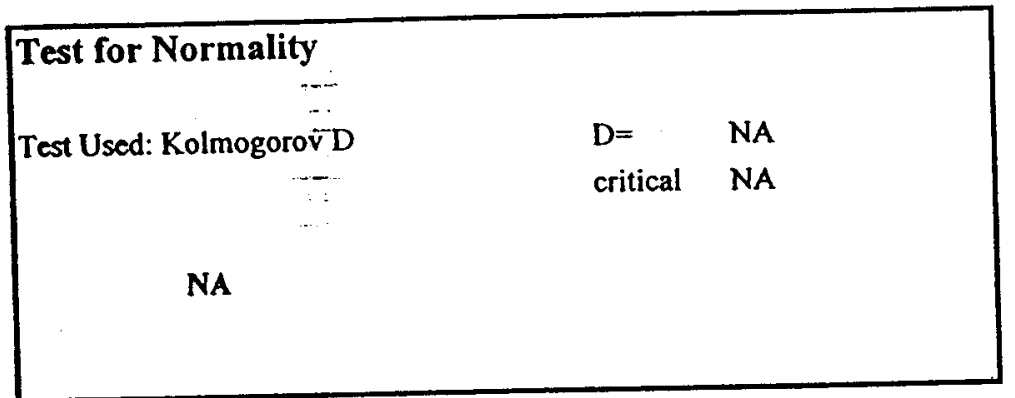

\begin{tabular}{|lr|}
\hline Test for Homogeneity of Variance & \\
Test Used: Bartlett's Test & B $=$ NA \\
NA & \\
\end{tabular}

\begin{tabular}{|c|c|c|c|c|c|c|}
\hline \multicolumn{7}{|c|}{ Test for Differences in Reproduction } \\
\hline Test Type Use & \multicolumn{6}{|c|}{$\begin{array}{l}\text { Non-Linear Regression - Logistic Model } \\
\text { Calculated Using JMP Software } \\
\text { \% Emfluent }\end{array}$} \\
\hline Enfoet & MHSF & $6.3 \%$ & $12.5 \%$ & $25.0 \%$ & $50.0 \%$ & $100 \%$ \\
\hline Survival & $90 \%$ & $100 \%$ & $100 \%$ & $100 \%$ & $90 \%$ & $90 \%$ \\
\hline Prodicled & $100 \%$ & $100 \%$ & $100 \%$ & $100 \%$ & $100 \%$ & $100 \%$ \\
\hline Reproduction & 24.9 & 25.5 & 27.2 & 29.0 & 24.6 & 25.5 \\
\hline Prodicted & 26.2 & 26.2 & 26.2 & 26.2 & 26.2 & 26.2 \\
\hline Verience & 24.7 & 17.39 & 19.07 & 11.50 & 91.60 & 32.28 \\
\hline \multirow{2}{*}{\multicolumn{4}{|c|}{ Teat Parameters }} & & $\begin{array}{l}\text { MSD- } \\
\text { PMSD }=\end{array}$ & $\begin{array}{r}5.95 \\
23.9 \%\end{array}$ \\
\hline & & & & \multirow{2}{*}{\multicolumn{2}{|c|}{$\begin{array}{l}\% \text { Reduction } \\
\text { CTC= } 100 \%\end{array}$}} & \\
\hline & Alpha & beta & gamma & & & Passfalil \\
\hline Survival & 3.00 & -200.00 & 0.00 & \multicolumn{2}{|c|}{$0.0 \%$} & Pass \\
\hline Reproduction & 4.78 & -199.00 & 26.16 & \multicolumn{2}{|c|}{$0.0 \%$} & Pass \\
\hline & IC25 $=$ & $>100 \%$ & & & & \\
\hline
\end{tabular}

Reproduction vs. Effluent Conc.

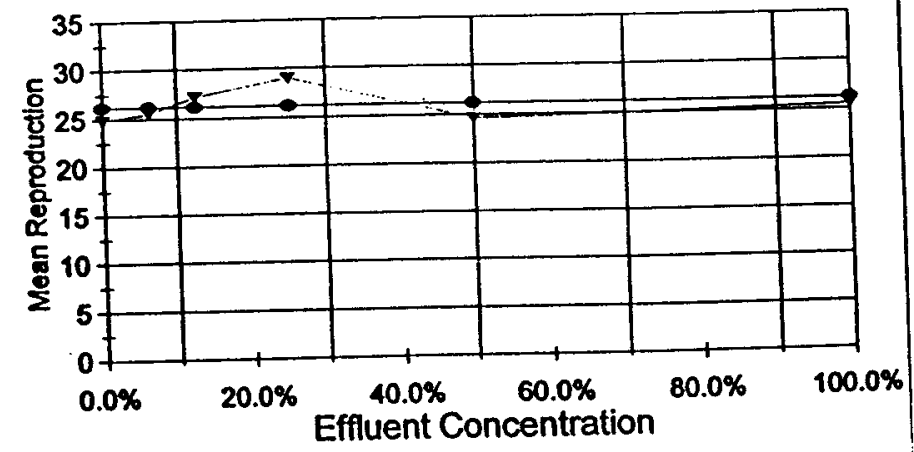

$\rightarrow$ Actual Reproduction

- Predicted Reproduction

\section{Summary}




\section{CHRONIC DEFINITIVE SURVIVAL AND REPRODUCTION TEST \\ Water Chemistry}

Client: WSRC

Sample Identification: A-01

Test Date: $\quad$ August 6, 2002

\section{pH (After Renewal)}

\begin{tabular}{|l|r|r|r|r|r|r|}
\hline \multicolumn{7}{|c|}{ Test Concentration } \\
\hline & Control & $6.25 \%$ & $12.5 \%$ & $25 \%$ & $50 \%$ & $100 \%$ \\
\hline Initial & 7.16 & 7.03 & 6.94 & 6.90 & 6.94 & 6.90 \\
\hline Day 1 & 7.20 & 7.02 & 6.99 & 6.97 & 6.90 & 6.87 \\
\hline Day 2 & 7.19 & 6.99 & 6.93 & 6.91 & 6.83 & 6.79 \\
\hline Day 3 & 7.29 & 7.06 & 6.99 & 6.98 & 6.91 & 6.93 \\
\hline Day 4 & 7.25 & 7.03 & 7.00 & 6.97 & 6.95 & 6.91 \\
\hline Day 5 & 7.17 & 7.05 & 7.03 & 6.95 & 6.91 & 6.83 \\
\hline Day 6 & 8.00 & 7.89 & 7.79 & 7.71 & 7.66 & \\
\hline
\end{tabular}

Dissolved Oxygen (After Renewal)

\begin{tabular}{|l|r|r|r|r|r|r|}
\hline & \multicolumn{7}{|c|}{ Test Concentration } \\
\hline Initial & 8.4 & 8.4 & 8.5 & 8.8 & 8.6 & 8.7 \\
\hline Day 1 & 8.4 & 8.5 & 8.5 & 8.6 & 8.6 & 8.6 \\
\hline Day 2 & 8.3 & 8.2 & 8.2 & 8.3 & 8.4 & 8.5 \\
\hline Day 3 & 8.4 & 8.3 & 8.3 & 8.3 & 8.5 & 8.6 \\
\hline Day 4 & 8.6 & 8.3 & 8.4 & 8.6 & 8.6 & 8.7 \\
\hline Day 5 & 8.4 & 8.3 & 8.4 & 8.5 & 8.5 & 8.6 \\
\hline Day 6 & 8.3 & 8.4 & 8.4 & 8.4 & 8.4 & 8.4 \\
\hline
\end{tabular}

Test Results Reviewed and Approved By:

pH (Before Renewal)
\begin{tabular}{|l|r|r|r|r|r|r|}
\hline \multicolumn{7}{|c|}{ Test Concentration } \\
\hline & Control & $6.25 \%$ & $12.5 \%$ & $25 \%$ & $50 \%$ & $100 \%$ \\
\hline Day 1 & 7.47 & 7.34 & 7.33 & 7.23 & 7.28 & 7.21 \\
\hline Day 2 & 7.53 & 7.02 & 7.00 & 6.99 & 6.97 & 6.93 \\
\hline Day 3 & 7.74 & 7.26 & 7.25 & 7.26 & 7.25 & 7.35 \\
\hline Day 4 & 7.39 & 7.19 & 7.17 & 7.16 & 7.11 & 7.15 \\
\hline Day 5 & 7.23 & 7.18 & 7.03 & 6.95 & 6.91 & 6.87 \\
\hline Day 6 & 8.37 & 8.24 & 8.14 & 8.10 & 8.00 & \\
\hline Final & & 8.20 & 6.91 & 6.96 & 6.96 & 7.26 \\
\hline
\end{tabular}

Dissolved Oxygen (Before Renewal)

\begin{tabular}{|l|r|r|r|r|r|r|}
\hline & Tentrol & $6.25 \%$ & $12.5 \%$ & $25 \%$ & $50 \%$ & $100 \%$ \\
\hline Day 1 & 8.4 & 8.4 & 8.4 & 8.5 & 8.5 & 8.6 \\
\hline Day 2 & 8.5 & 8.5 & 8.5 & 8.6 & 8.6 & 8.7 \\
\hline Day 3 & 8.5 & 8.5 & 8.6 & 8.6 & 8.6 & 8.6 \\
\hline Day 4 & 8.5 & 8.6 & 8.7 & 8.7 & 8.8 & 8.8 \\
\hline Day 5 & 8.6 & 8.7 & 8.7 & 0.7 & 8.8 & 8.8 \\
\hline Day 6 & 8.4 & 8.3 & 8.4 & 8.4 & 8.5 & 8.6 \\
\hline Final & & 8.2 & 8.1 & 7.9 & 7.8 & 7.7 \\
\hline
\end{tabular}




\section{Day Chronic Definitive Survival and Reproduction Bioassay Method: EPA600/4-91/002}

Test Organism:

Daphnia ambigua

Facility: WSRC

Sample ID: A-11

Aiken County

ETT\#: 20334

Sample ID: August 6, 2002 


\section{CHRONIC DEFINITIVE SURVIVAL AND REPRODUCTION TEST Survival and Reproduction Results}

Client: WSRC

Sample Identification: A-11

Test Date:

August 6, 2002

\begin{tabular}{|c|c|c|c|c|c|c|}
\hline \multicolumn{7}{|c|}{ Reproduction at } \\
\hline Rep. & Day 3/4 & Day 5 & Day 6 & Day 7 & Day 8 & Total \\
\hline 1 & 4 & 0 & 9 & 0 & 16 & 29 \\
\hline 2 & 0 & 5 & 11 & 0 & 15 & 31 \\
\hline 3 & 0 & 4 & 8 & 0 & 11 & 23 \\
\hline 4 & 0 & 4 & 9 & 0 & 14 & 27 \\
\hline 5 & 0 & 6 & 9 & 0 & 12 & 27 \\
\hline 6 & 0 & 5 & 10 & 0 & 13 & 28 \\
\hline 7 & 0 & 3 & 8 & 0 & 14 & 25 \\
\hline 8 & 0 & 4 & 10 & 0 & 12 & 26 \\
\hline 9 & 5 & 0 & 10 & $X$ & $X$ & 15 \\
\hline 10 & 2 & 4 & .12 & 0 & 0 & 18 \\
\hline
\end{tabular}

\begin{tabular}{|c|c|c|c|c|c|c|}
\hline \multicolumn{1}{|c}{ Reproduction at } & \multicolumn{3}{c|}{$12.5 \%$} & \multicolumn{1}{c|}{ Concentration } \\
\hline Rep. & Day 3/4 & Day 5 & Day 6 & Day 7 & Day 8 & Total \\
\hline 1 & 4 & 0 & 13 & 0 & 10 & 27 \\
\hline 2 & 0 & 5 & 8 & 0 & 9 & 22 \\
\hline 3 & 0 & 4 & 8 & 0 & 12 & 24 \\
\hline 4 & 0 & 3 & 8 & 0 & 9 & 20 \\
\hline 5 & 0 & 6 & 10 & 0 & 10 & 26 \\
\hline 6 & 0 & 6 & 13 & 0 & 12 & 31 \\
\hline 7 & 0 & 6 & 11 & 0 & 13 & 30 \\
\hline 8 & 0 & 4 & 9 & 0 & 10 & 23 \\
\hline 9 & 2 & 0 & 9 & 0 & 13 & 24 \\
\hline 10 & 0 & 3 & 9 & 0 & 9 & 21 \\
\hline
\end{tabular}

\begin{tabular}{|c|c|c|c|c|c|c|}
\hline \multicolumn{1}{|c|}{ Reproduction at } & \multicolumn{3}{c|}{$50 \%$} & Concentration \\
\hline Rep. & Day 3/4 & Day 5 & Day 6 & Day 7 & Day 8 & Total \\
\hline 1 & 3 & 0 & 9 & 0 & 14 & 26 \\
\hline 2 & 0 & 4 & 15 & 0 & 13 & 32 \\
\hline 3 & 4 & 0 & 9 & 0 & 13 & 26 \\
\hline 4 & 0 & 5 & 10 & 0 & 12 & 27 \\
\hline 5 & 0 & 7 & 12 & 0 & 14 & 33 \\
\hline 6 & 0 & 6 & 13 & 0 & 15 & 34 \\
\hline 7 & 0 & 5 & 8 & 0 & 14 & 27 \\
\hline 8 & 0 & 5 & 14 & 0 & 12 & 31 \\
\hline 9 & 0 & 4 & 13 & 0 & 12 & 29 \\
\hline 10 & 3 & 0 & 13 & 12 & 0 & 28 \\
\hline
\end{tabular}

County:

NPDES\#: $\quad$ SC

ETT \#:

20334

\begin{tabular}{|c|c|c|c|c|c|c|}
\hline \multicolumn{2}{|c|}{ Reproduction at } & \multicolumn{2}{c|}{$6.25 \%$} & \multicolumn{2}{c|}{ Concentration } \\
\hline Rep. & Day 3/4 & Day 5 & Day 6 & Day 7 & Day 8 & Total \\
\hline 1 & 0 & 4 & 11 & 0 & 13 & 28 \\
\hline 2 & 0 & 3 & 10 & 0 & 15 & 28 \\
\hline 3 & 4 & 0 & 9 & 0 & 10 & 23 \\
\hline 4 & 0 & 5 & 13 & 0 & 12 & 30 \\
\hline 5 & 0 & 4 & 12 & 0 & 15 & 31 \\
\hline 6 & 0 & 5 & 11 & 0 & 14 & 30 \\
\hline 7 & 0 & 2 & 12 & 0 & 11 & 25 \\
\hline 8 & 0 & 5 & 10 & 0 & 12 & 27 \\
\hline 9 & 0 & 4 & 9 & 0 & 12 & 25 \\
\hline 10 & 0 & 5 & 8 & 0 & 11 & 24 \\
\hline
\end{tabular}

\begin{tabular}{|c|c|c|c|c|c|c|}
\hline \multicolumn{2}{|c|}{ Reproduction at } & \multicolumn{2}{c|}{$25 \%$} & \multicolumn{2}{c|}{ Concentration } \\
\hline Rep. & Day 3/4 & Day 5 & Day 6 & Day 7 & Day 8 & Total \\
\hline 1 & 2 & 0 & 9 & 0 & 11 & 22 \\
\hline 2 & 0 & 5 & 11 & 0 & 11 & 27 \\
\hline 3 & 4 & 0 & 12 & 0 & 13 & 29 \\
\hline 4 & 0 & 4 & 9 & 0 & 12 & 25 \\
\hline 5 & 0 & 4 & 13 & 0 & 14 & 31 \\
\hline 6 & 0 & 6 & 13 & 0 & 14 & 33 \\
\hline 7 & 0 & 4 & 8 & 0 & 11 & 23 \\
\hline 8 & 0 & 4 & 10 & 0 & 13 & 27 \\
\hline 9 & 0 & 5 & 10 & 0 & 16 & 31 \\
\hline 10 & 4 & 0 & 11 & 0 & 12 & 27 \\
\hline
\end{tabular}

\begin{tabular}{|c|c|c|c|c|c|c}
\hline Reproduction at & \multicolumn{4}{c}{$100 \%$} & \multicolumn{2}{c}{ Concentration } \\
\hline Rep. & Day 3/4 & Day 5 & Day 6 & Day 7 & Day 8 & Tocal \\
\hline 1 & 0 & 4 & 13 & 0 & 10 & 27 \\
\hline 2 & 0 & 5 & 14 & 0 & 14 & 33 \\
\hline 3 & 3 & 0 & 11 & 0 & 13 & 27 \\
\hline 4 & 2 & 0 & 13 & 0 & 12 & 27 \\
\hline 5 & 0 & 3 & 8 & 0 & 14 & 25 \\
\hline 6 & 0 & 5 & 10 & 0 & 9 & 24 \\
\hline 7 & 0 & 4 & 13 & 0 & 11 & 28 \\
\hline 8 & 0 & 6 & 10 & 0 & 9 & 25 \\
\hline 9 & 0 & 5 & 10 & 0 & 14 & 29 \\
\hline 10 & 0 & 5 & 9 & 0 & 0 & 14 \\
\hline
\end{tabular}




\section{CHRONIC DEFINITIVE SURVIVAL AND REPRODUCTION TEST \\ Statistical Analyses}

Client:

Sample Identification:

Test Date:
WSRC

A-11

August 6, 2002

\section{Test for Normality}

Test Used: Kolmogoröv D

$\begin{array}{ll}D= & N A \\ \text { critical } & N A\end{array}$

NA
Test for Homogeneity of Variance

Test Used: Bartlett's Test

$B=N A$

critical NA

NA

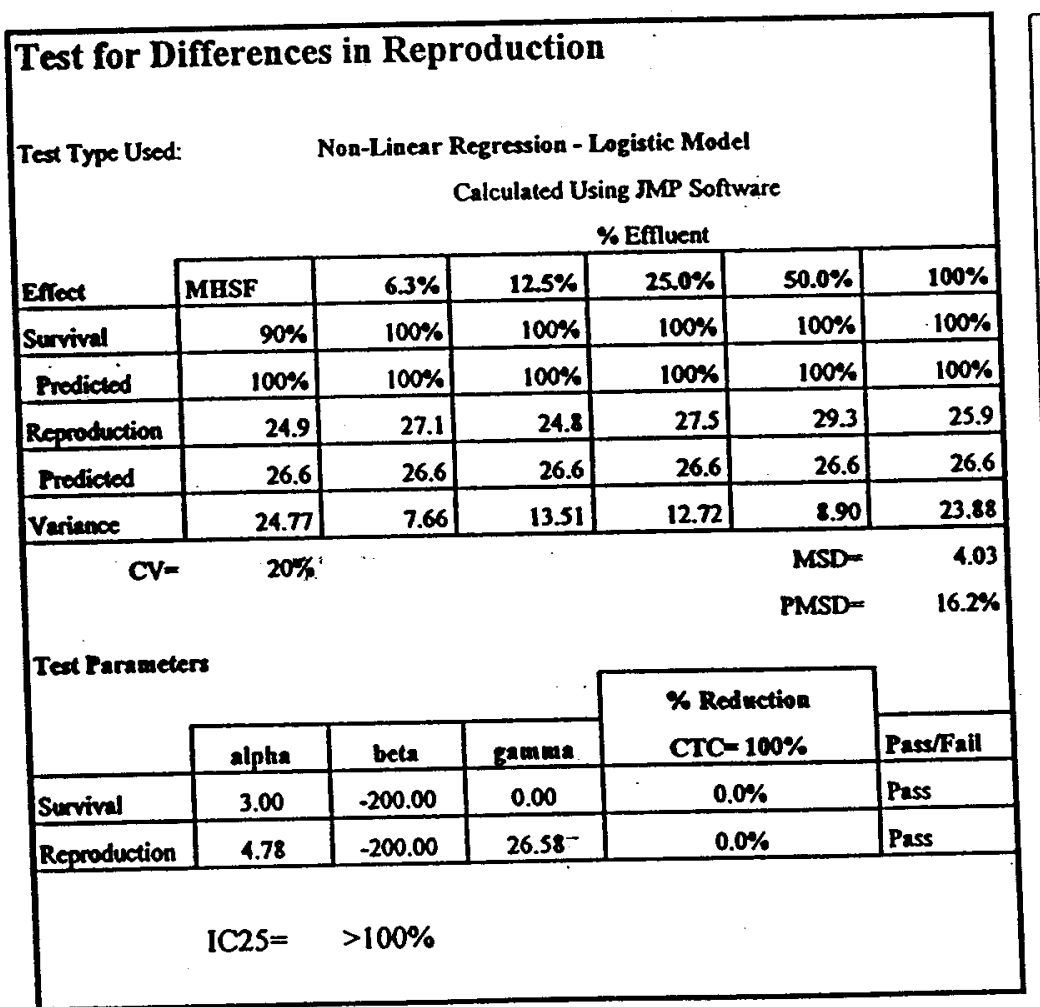

Reproduction vs. Effluent Conc.

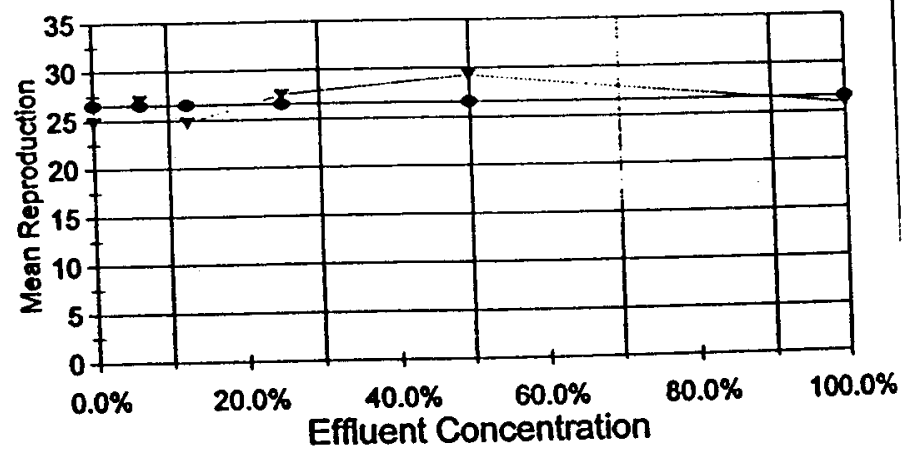

$\rightarrow$ - Actual Reproduction $\rightarrow$ Predicted Reproduction

Summary

The reductions in survival and reproduction at the CTC are $<25 \%$ - Pass 


\section{CHRONIC DEEFINITIVE SURVIVAL AND REPRODUCTION TEST \\ Water Chemistry}

Client: WSRC

Sample Identification: A-11

Test Date: $\quad$ August 6, 2002

pH (After Renewal)

\begin{tabular}{|l|r|r|r|r|r|r|}
\hline \multicolumn{7}{|c|}{ Test Concentration } \\
\hline & Control & $6.25 \%$ & $12.5 \%$ & $25 \%$ & $50 \%$ & $100 \%$ \\
\hline Initial & 7.16 & 7.10 & 7.02 & 6.94 & 6.99 & 7.00 \\
\hline Day 1 & 7.20 & 7.39 & 7.38 & 7.28 & 7.22 & 7.22 \\
\hline Day 2 & 7.19 & 7.42 & 7.40 & 7.39 & 7.25 & 7.21 \\
\hline Day 3 & 7.29 & 7.44 & 7.35 & 7.22 & 7.18 & 7.70 \\
\hline Day 4 & 7.25 & 7.30 & 7.53 & 7.51 & 7.49 & 7.30 \\
\hline Day 5 & 7.17 & 7.21 & 7.13 & 7.09 & 6.95 & 7.01 \\
\hline Day 6 & 8.00 & 7.66 & 7.57 & 7.44 & 7.41 & 7.41 \\
\hline
\end{tabular}

Dissolved Oxygen (After Renewal)

\begin{tabular}{|l|r|r|r|r|r|r|}
\hline & \multicolumn{7}{|c|}{ Test Concentration } \\
\hline Initial & 8.4 & 8.2 & 8.2 & 8.3 & 8.4 & 8.4 \\
\hline Day 1 & 8.4 & 8.1 & 8.2 & 8.2 & 8.3 & 8.4 \\
\hline Day 2 & 8.3 & 8.0 & 8.3 & 8.3 & 8.4 & 8.4 \\
\hline Day 3 & 8.4 & 8.3 & 8.4 & 8.3 & 8.6 & 8.6 \\
\hline Day 4 & 8.6 & 8.2 & 8.3 & 8.3 & 8.4 & 8.4 \\
\hline Day 5 & 8.4 & 8.1 & 8.2 & 8.3 & 8.3 & 8.4 \\
\hline Day 6 & 8.3 & 8.2 & 8.2 & 8.3 & 8.3 & 8.3 \\
\hline
\end{tabular}

Test Results Reviewed and Approved By:
pH (Before Renewal)

\begin{tabular}{|l|r|r|r|r|r|r|}
\hline \multicolumn{7}{|c|}{ Test Concentration } \\
\hline & Control & $6.25 \%$ & $12.5 \%$ & $25 \%$ & $50 \%$ & $100 \%$ \\
\hline Day 1 & 7.47 & 7.59 & 7.61 & 7.55 & 7.52 & 7.51 \\
\hline Day 2 & 7.53 & 7.63 & 7.60 & 7.49 & 7.41 & 7.40 \\
\hline Day 3 & 7.74 & 7.40 & 7.63 & 7.58 & 7.55 & 7.51 \\
\hline Day 4 & 7.39 & 7.59 & 7.51 & 7.33 & 7.49 & 7.33 \\
\hline Day 5 & 7.23 & 7.69 & 7.63 & 7.59 & 7.60 & 7.61 \\
\hline Day 6 & 8.37 & 7.65 & 7.81 & 7.77 & 7.70 & 7.77 \\
\hline Final & & 7.65 & 7.54 & 7.43 & 7.45 & 7.43 \\
\hline
\end{tabular}

Dissolved Oxygen (Before Renewal)

\begin{tabular}{|l|r|r|r|r|r|r|}
\hline \multicolumn{7}{|c|}{ Test Concentration } \\
\hline & Control & $6.25 \%$ & $12.5 \%$ & $25 \%$ & $50 \%$ & $100 \%$ \\
\hline Day 1 & 8.4 & 8.2 & 8.2 & 8.4 & 8.4 & 8.4 \\
\hline Day 2 & 8.5 & 8.3 & 8.3 & 8.3 & 8.4 & 8.4 \\
\hline Day 3 & 8.5 & 8.3 & 8.4 & 8.5 & 8.7 & 8.7 \\
\hline Day 4 & 8.5 & 8.4 & 8.4 & 8.5 & 8.6 & 8.7 \\
\hline Day 5 & 8.6 & 8.3 & 8.4 & 8.4 & 8.6 & 8.7 \\
\hline Day 6 & 8.4 & 8.2 & 8.2 & 8.3 & 8.4 & 8.5 \\
\hline Final & & & & & & \\
\hline
\end{tabular}

Temperature

\begin{tabular}{|l|r|}
\hline \multicolumn{2}{|c|}{ Incubator ${ }^{\circ} \mathrm{C}$} \\
\hline Initial & 24.4 \\
\hline Day 1 & 24.3 \\
\hline Day 2 & 24.1 \\
\hline Day 3 & 24.3 \\
\hline Day 4 & 24.3 \\
\hline Day 5 & 24.4 \\
\hline Day 6 & 24.5 \\
\hline Final & 25.8 \\
\hline
\end{tabular}


7 Day Chronic Definitive Survival and Reproduction Bioassay

Method: EPA/600/4-91/002

Test Organism:

Daphnia ambigua

Facility: WSRC

Sample ID: G-10

Aiken County

ETT\#: 20335

Sample ID: August 6, 2002 


\begin{tabular}{|c|c|c|c|c|c|c|}
\hline 6 & 0 & 6 & 10 & 0 & 13 & 29 \\
\hline 7 & 0 & 5 & 8 & 0 & 12 & 25 \\
\hline 8 & 0 & 4 & 10 & 0 & 12 & 26 \\
\hline 9 & 0 & 5 & 11 & 0 & 13 & 29 \\
\hline 10 & 4 & 0 & 6 & 14 & 0 & 24 \\
\hline
\end{tabular}

\begin{tabular}{|c|c|c|c|c|c|c|}
\hline 6 & 0 & 6 & 10 & 0 & 9 & 25 \\
\hline 7 & 5 & 2 & 11 & 0 & 14 & 32 \\
\hline 8 & 0 & 5 & 6 & 11 & 0 & 22 \\
\hline 9 & 0 & 6 & 9 & 0 & 12 & 27 \\
\hline 10 & 4 & 0 & 8 & 14 & 0 & 26 \\
\hline
\end{tabular}

\begin{tabular}{|c|c|c|c|c|c|c|}
\hline \multicolumn{2}{|c|}{ Reproduction at } & \multicolumn{3}{c|}{$12.5 \%$} & \multicolumn{3}{c|}{ Concentration } \\
\hline Rep. & Day 344 & Day 5 & Day 6 & Day 7 & Day 8 & Total \\
\hline 1 & 5 & 0 & 7 & 14 & 0 & 26 \\
\hline 2 & 0 & 4 & 10 & 0 & 14 & 28 \\
\hline 3 & 5 & 0 & 11 & 13 & 0 & 29 \\
\hline 4 & 4 & 0 & 8 & 0 & 10 & 22 \\
\hline 5 & 0 & 5 & 10 & 0 & 14 & 29 \\
\hline 6 & 0 & 6 & 7 & 0 & 13 & 26 \\
\hline 7 & 0 & 4 & 7 & 0 & 11 & 22 \\
\hline 8 & 0 & 4 & 10 & 0 & 14 & 28 \\
\hline 9 & 0 & 5 & 7 & 0 & 11 & 23 \\
\hline 10 & 4 & 0 & 10 & 14 & 0 & 28 \\
\hline
\end{tabular}

\begin{tabular}{|c|c|c|c|c|c|c|}
\hline \multicolumn{2}{|c|}{ Reproduction at } & \multicolumn{2}{c|}{$25 \%$} & \multicolumn{2}{c|}{ Concentration } \\
\hline Rep. & Day 3/4 & Day 5 & Day 6 & Day 7 & Day 8 & Total \\
\hline 1 & 4 & 0 & 8 & 0 & 10 & 22 \\
\hline 2 & 0 & 4 & 12 & 0 & 13 & 29 \\
\hline 3 & 6 & 0 & 11 & 12 & 0 & 29 \\
\hline 4 & 0 & 3 & 7 & 0 & 14 & 24 \\
\hline 5 & 0 & 5 & 9 & 0 & 16 & 30 \\
\hline 6 & 0 & 5 & 9 & 0 & 14 & 28 \\
\hline 7 & 0 & 4 & 7 & 0 & 12 & 23 \\
\hline 8 & 0 & 3 & 10 & 0 & 13 & 26 \\
\hline 9 & 0 & 4 & 5 & 0 & 11 & 20 \\
\hline 10 & 0 & 5 & 0 & 0 & 9 & 23 \\
\hline
\end{tabular}

\begin{tabular}{|c|c|c|c|c|c|c|}
\hline \multicolumn{2}{|c|}{ Reproduction at } & \multicolumn{4}{c|}{$50 \%$} & Concentration \\
\hline Rep. & Day 3/4 & Day 5 & Day 6 & Day 7 & Day 8 & Total \\
\hline 1 & 0 & 4 & 11 & 0 & 11 & 26 \\
\hline 2 & 0 & 5 & 9 & 0 & 13 & 27 \\
\hline 3 & 5 & 0 & 10 & 15 & 0 & 30 \\
\hline 4 & 4 & 0 & 11 & 13 & 0 & 28 \\
\hline 5 & 0 & 4 & 11 & 0 & 13 & 28 \\
\hline 6 & 0 & 4 & 11 & 0 & 13 & 28 \\
\hline 7 & 0 & 5 & 11 & 0 & 9 & 25 \\
\hline 8 & 0 & 3 & $X$ & $X$ & $X$ & 3 \\
\hline 9 & 0 & 4 & 1 & 9 & 9 & 23 \\
\hline 10 & 4 & $X$ & $X$ & $X$ & $X$ & 4 \\
\hline
\end{tabular}

\begin{tabular}{|c|c|c|c|c|c|c}
\hline Reproduction at & \multicolumn{2}{c}{$100 \%$} & \multicolumn{2}{c}{ Concentration } \\
\hline Rep. & Day 3/4 & Day 5 & Day 6 & Day 7 & Day 8 & Total \\
\hline 1 & 4 & 0 & 8 & 0 & 12 & 24 \\
\hline 2 & 0 & 3 & $X$ & $X$ & $X$ & 3 \\
\hline 3 & 5 & 0 & 7 & 0 & 9 & 21 \\
\hline 4 & 4 & 0 & 12 & 0 & 10 & 26 \\
\hline 5 & 0 & 4 & 10 & 0 & 13 & 27 \\
\hline 6 & 0 & 3 & 9 & 0 & 8 & 20 \\
\hline 7 & 0 & 4 & 9 & 0 & 9 & 22 \\
\hline 8 & 0 & 4 & 10 & 0 & 11 & 25 \\
\hline 9 & 0 & 4 & $X$ & $X$ & $X$ & 4 \\
\hline 10 & 5 & 0 & 9 & 13 & 0 & 27 \\
\hline
\end{tabular}




\section{CHRONIC DEFINITIVE SURVIVAL AND REPRODUCTION TEST \\ Statistical Analyses}

Client:

WSRC

Sample Identification:

G-10

Test Date:

August 6, 2002

\begin{tabular}{|lll}
\hline Test for Normality & & \\
Test Used: Kolmogorov D & $D=$ & NA \\
& critical & NA
\end{tabular}

NA

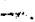

Test for Differences in Reproduction

Test Type Used: $\quad$ Non-Liacar Regression - Lozintic Modd

Calculared Using JMP Soltware

\begin{tabular}{|c|c|c|c|c|c|c|}
\hline \multirow[b]{2}{*}{ Erfect } & \multicolumn{6}{|c|}{$*$ Eflicen } \\
\hline & MHSF & $6.3 \%$ & $12.5 \%$ & $25.0 \%$ & $\sec x$ & $100 \%$ \\
\hline Sunival & $90 \%$ & $100 \%$ & $100 \%$ & $100 \%$ & 8006 & $80 \%$ \\
\hline Inodicaed & $100 \%$ & $100 \%$ & $100 \%$ & $100 \%$ & $100 x$ & $100 \%$ \\
\hline Reproduction & 24.9 & 273 & 26.1 & 25.4 & 22.2 & 19.9 \\
\hline Prodicted & 262 & 26.0 & 25.7 & 24.9 & 232 & 19.6 \\
\hline Verience & 57.88 & . 7.79 & 7.28 & 12.04 & 100.84 & e.s. \\
\hline C & $31 \%$ & & & & $\begin{array}{l}\text { MSD }= \\
\text { MMLD }=\end{array}$ & $27.7 \%$ \\
\hline
\end{tabular}

Tet Parameters

\begin{tabular}{|c|c|c|c|c|c|c|}
\hline \multicolumn{4}{|c|}{ 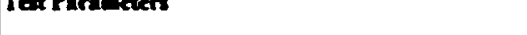 } & \multirow{2}{*}{\multicolumn{2}{|c|}{$\begin{array}{c}\text { K Redection } \\
\text { CTC-19K = 1COK }\end{array}$}} & \multirow{3}{*}{ Panerfall } \\
\hline & slphe & beta & semana & & & \\
\hline Sonvival & -3.00 & .200 .00 & 0.00 & $0.0 \%$ & $0.0 x$ & \\
\hline Reproduction & 4.78 & -20200 & 22.17 & $3.4 x$ & $25.2 \%$ & Pass \\
\hline
\end{tabular}

$1025=$

$98.8 \%$
Test for Homogeneity of Variance

Test Used: Bartlett's Test

$B=N A$

critical NA

NA

\section{Reproduction vs. Effluent Conc.}

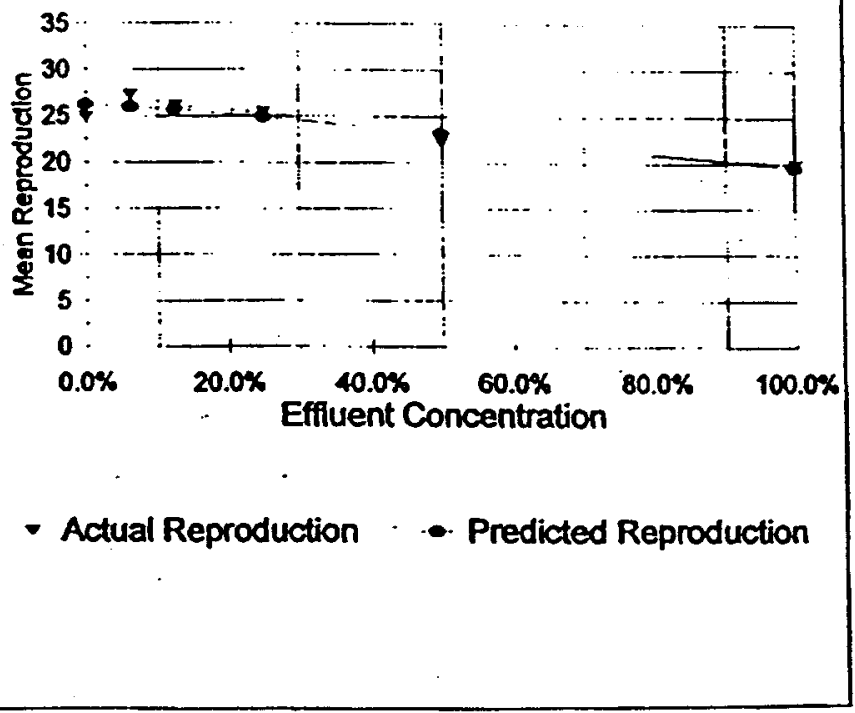

Summary

The reductions in survival and reproduction at the CTC are $<5 \%$ - Pass 


\section{CHRONIC DEFINITIVE SURVIVAL AND REPRODUCTION TEST \\ Water Chemistry}

Client:

Sample Identification:

Test Date:

WSRC

August 6, 2002
pH (After Renewal)

\begin{tabular}{|l|r|r|r|r|r|r|}
\hline & \multicolumn{7}{|c|}{ Test Concentration } \\
\hline & Control & $6.25 \%$ & $12.5 \%$ & $25 \%$ & $50 \%$ & $100 \%$ \\
\hline Initial & 6.91 & 7.12 & 7.27 & 7.47 & 7.65 & 8.03 \\
\hline Day 1 & 7.57 & 7.35 & 7.35 & 7.91 & 7.75 & 8.04 \\
\hline Day 2 & 7.49 & 7.29 & 7.31 & 7.42 & 7.45 & 7.53 \\
\hline Day 3 & 7.69 & 7.21 & 7.15 & 7.26 & 7.27 & 7.39 \\
\hline Day 4 & 7.51 & 7.36 & 7.33 & 7.31 & 7.29 & 7.19 \\
\hline Day 5 & 7.23 & 7.35 & 7.36 & 7.39 & 7.41 & 7.55 \\
\hline Day 6 & 9.23 & 8.13 & 7.90 & 7.78 & 7.76 & 7.88 \\
\hline
\end{tabular}

Dissolved Oxygen (After Renewal)

\begin{tabular}{|l|r|r|r|r|r|r|}
\hline & Control & $6.25 \%$ & $12.5 \%$ & $25 \%$ & $50 \%$ & $100 \%$ \\
\hline Initial & 8.0 & 8.2 & 8.2 & 8.3 & 8.3 & 8.4 \\
\hline Day 1 & 8.3 & 8.2 & 8.2 & 8.0 & 8.3 & 8.5 \\
\hline Day 2 & 8.2 & 8.6 & 8.3 & 8.4 & 8.5 & 8.6 \\
\hline Day 3 & 8.3 & 8.2 & 8.2 & 8.3 & 8.3 & 8.4 \\
\hline Day 4 & 8.2 & 8.3 & 8.4 & 8.5 & 8.5 & 8.5 \\
\hline Day 5 & 8.3 & 8.3 & 8.3 & 8.3 & 8.3 & 8.4 \\
\hline Day 6 & 8.0 & 8.2 & 8.3 & 8.3 & 8.4 & 8.6 \\
\hline
\end{tabular}

Test Results Reviewed and Approved By:

pH (Before Renewal)
\begin{tabular}{|l|r|r|r|r|r|r|}
\hline & Control & $6.25 \%$ & $12.5 \%$ & $25 \%$ & $50 \%$ & $100 \%$ \\
\hline Day 1 & 7.60 & 7.53 & 7.43 & 7.50 & 7.89 & 8.14 \\
\hline Day 2 & 7.59 & 7.47 & 7.54 & 7.69 & 7.77 & 7.81 \\
\hline Day 3 & 7.86 & 7.63 & 7.60 & 7.63 & 7.67 & 7.71 \\
\hline Day 4 & 7.91 & 7.51 & 7.49 & 7.43 & 7.39 & 7.46 \\
\hline Day 5 & 7.51 & 7.45 & 7.83 & 7.89 & 7.89 & 8.09 \\
\hline Day 6 & 7.43 & 7.36 & 7.93 & 7.53 & 7.51 & 7.79 \\
\hline Final & 7.26 & 7.02 & 6.99 & 7.14 & 7.38 & 7.64 \\
\hline
\end{tabular}

Dissolved Oxygen (Before Renewal)

\begin{tabular}{|l|r|r|r|r|r|r|}
\hline & Control & $6.25 \%$ & $12.5 \%$ & $25 \%$ & $50 \%$ & $100 \%$ \\
\hline Day 1 & 8.2 & 8.2 & 8.2 & 8.2 & 8.3 & 8.4 \\
\hline Day 2 & 8.1 & 8.5 & 8.3 & 8.4 & 8.4 & 8.5 \\
\hline Day 3 & 8.1 & 8.2 & 8.2 & 8.3 & 8.4 & 8.5 \\
\hline Day 4 & 8.4 & 8.3 & 8.3 & 8.3 & 8.4 & 8.4 \\
\hline Day 5 & 8.2 & 8.3 & 8.3 & 8.4 & 8.4 & 8.5 \\
\hline Day 6 & 8.2 & 8.1 & 8.2 & 8.2 & 8.3 & 8.3 \\
\hline Final & 7.8 & 7.8 & 7.7 & 7.7 & 7.7 & 7.8 \\
\hline
\end{tabular}

Temperature
\begin{tabular}{|l|r|}
\hline \multicolumn{1}{|c|}{ Incubator ${ }^{\circ} \mathrm{C}$} \\
\hline Initial & 24.4 \\
\hline Day 1 & 24.3 \\
\hline Day 2 & 24.1 \\
\hline Day 3 & 24.3 \\
\hline Day 4 & 24.3 \\
\hline Day 5 & 24.4 \\
\hline Day 6 & 24.5 \\
\hline Final & 25.8 \\
\hline
\end{tabular}




\section{Day Chronic Definitive Survival and Reproduction Bioassay \\ Method: EPA/600/4-91/002}

-

$-$

$-$

Test Organism:

Daphnia ambigua

Facility: WSRC

Sample ID: $\mathrm{A}-01$

County

ETT\#: 20569

Sample ID: September 10, 2002 


\section{CHRONIC DEFINITIVE SURVIVAL AND REPRODUCTION TEST Survival and Reproduction Results}

Client:

Sample Identification:

Test Date:

\begin{tabular}{|c|c|c|c|c|c|c|}
\hline \multicolumn{2}{|c|}{ Reproduction at } & \multicolumn{7}{c|}{ MHSF } & Control \\
\hline Rep. & Day 3/4 & Day 5 & Day 6 & Day 7 & Day 8 & Total \\
\hline 1 & 0 & 5 & 8 & 0 & 10 & 23 \\
\hline 2 & 0 & 3 & 7 & 0 & 12 & 22 \\
\hline 3 & 0 & 5 & 5 & 0 & 11 & 21 \\
\hline 4 & 0 & 4 & 5 & 0 & 13 & 22 \\
\hline 5 & 4 & 0 & 5 & 9 & 0 & 18 \\
\hline 6 & 0 & 5 & 0 & 9 & 13 & 27 \\
\hline 7 & 5 & 0 & 6 & 11 & 0 & 22 \\
\hline 8 & 0 & 4 & 0 & 7 & 10 & 21 \\
\hline 9 & 0 & 4 & 0 & 7 & 11 & 22 \\
\hline 10 & 0 & 4 & 7 & 0 & 13 & 24 \\
\hline
\end{tabular}

\begin{tabular}{|c|c|c|c|c|c|c|}
\hline Reproduction at & \multicolumn{4}{c|}{$12.5 \%$} & \multicolumn{3}{c|}{ Concentration } \\
\hline Rep. & Day 3/4 & Day 5 & Day 6 & Day 7 & Day 8 & Total \\
\hline 1 & 3 & 0 & 7 & 12 & 0 & 22 \\
\hline 2 & 4 & 0 & 8 & 10 & 0 & 22 \\
\hline 3 & 5 & 0 & 7 & 8 & 0 & 20 \\
\hline 4 & 0 & 4 & 5 & 0 & 12 & 21 \\
\hline 5 & 3 & 0 & 6 & 10 & 0 & 19 \\
\hline 6 & 0 & 5 & 6 & 0 & 13 & 24 \\
\hline 7 & 0 & 5 & 1 & 10 & 14 & 30 \\
\hline 8 & 0 & 4 & 8 & 0 & 14 & 26 \\
\hline 9 & 3 & 0 & 8 & 10 & 0 & 21 \\
\hline 10 & 0 & 5 & 9 & 0 & 13 & 27 \\
\hline
\end{tabular}

\begin{tabular}{|c|c|c|c|c|c|c|}
\hline Reproduction at & \multicolumn{3}{c|}{$50 \%$} & Concentration \\
\hline Rep. & Day 3/4 & Day 5 & Day 6 & Day 7 & Day 8 & Total \\
\hline 1 & 3 & 0 & 7 & 11 & 0 & 21 \\
\hline 2 & 4 & 1 & 6 & 12 & 0 & 23 \\
\hline 3 & 1 & 5 & 5 & 0 & 13 & 24 \\
\hline 4 & 0 & 3 & 0 & 0 & 12 & 15 \\
\hline 5 & 4 & 0 & 8 & 12 & 0 & 24 \\
\hline 6 & 3 & 0 & 5 & 13 & 0 & 21 \\
\hline 7 & 3 & 0 & 6 & 14 & 0 & 23 \\
\hline 8 & 0 & 5 & 7 & 0 & 12 & 24 \\
\hline 9 & 0 & 3 & 7 & 0 & 14 & 24 \\
\hline 10 & 0 & 5 & 8 & 0 & 13 & 26 \\
\hline
\end{tabular}

County:

NPDES\#: $\quad$ SC

ETT \#:

20569

\begin{tabular}{|c|c|c|c|c|c|c|}
\hline \multicolumn{2}{|c|}{ Reproduction at } & \multicolumn{2}{c|}{$6.25 \%$} & \multicolumn{2}{c|}{ Concentration } \\
\hline Rep. & Day 3/4 & Day 5 & Day 6 & Day 7 & Day 8 & Total \\
\hline 1 & 3 & 0 & 9 & 12 & 0 & 24 \\
\hline 2 & 0 & 4 & 6 & 0 & 11 & 21 \\
\hline 3 & 4 & 0 & 8 & 14 & 0 & 26 \\
\hline 4 & 0 & 2 & 6 & 0 & 13 & 21 \\
\hline 5 & 2 & 0 & 5 & 8 & 0 & 15 \\
\hline 6 & 0 & 4 & 5 & 0 & 12 & 21 \\
\hline 7 & 3 & 0 & 9 & 15 & 0 & 27 \\
\hline 8 & 0 & 3 & 7 & 0 & 13 & 23 \\
\hline 9 & 0 & 0 & 5 & 10 & 0 & 15 \\
\hline 10 & 0 & 4 & 5 & 0 & 12 & 21 \\
\hline
\end{tabular}

\begin{tabular}{|c|c|c|c|c|c|c}
\hline \multicolumn{2}{|c}{ Reproduction at } & \multicolumn{2}{c}{$25 \%$} & \multicolumn{2}{c}{ Concentration } \\
\hline Rep. & Day 3/4 & Day 5 & Day 6 & Day 7 & Day 8 & Total \\
\hline 1 & 4 & 0 & 7 & 12 & 0 & 23 \\
\hline 2 & 0 & 3 & 7 & 0 & 10 & 20 \\
\hline 3 & 3 & 0 & 7 & 0 & 12 & 22 \\
\hline 4 & 0 & 4 & 8 & $X$ & $X$ & 12 \\
\hline 5 & 4 & 0 & 7 & 11 & 0 & 22 \\
\hline 6 & 3 & 0 & 7 & 11 & 0 & 21 \\
\hline 7 & 4 & 0 & 5 & 13 & 0 & 22 \\
\hline 8 & 0 & 3 & 3 & 0 & 12 & 18 \\
\hline 9 & 0 & 5 & 9 & 0 & 11 & 25 \\
\hline 10 & 0 & 5 & 7 & 0 & 12 & 24 \\
\hline
\end{tabular}

\begin{tabular}{|c|c|c|c|c|c|c}
\hline \multicolumn{2}{|c}{ Reproduction at } & \multicolumn{2}{c}{$100 \%$} & \multicolumn{2}{c}{ Concentration } \\
\hline Rep. & Day 3/4 & Day 5 & Day 6 & Day 7 & Day 8 & Total \\
\hline 1 & 3 & 0 & 9 & 13 & 0 & 25 \\
\hline 2 & 0 & 2 & 7 & 0 & 14 & 23 \\
\hline 3 & 0 & 6 & 7 & 0 & 13 & 26 \\
\hline 4 & 0 & 3 & 8 & 0 & 12 & 23 \\
\hline 5 & 1 & 0 & 5 & 8 & 2 & 16 \\
\hline 6 & 3 & 0 & 7 & 10 & 0 & 20 \\
\hline 7 & 3 & 0 & 8 & 13 & 0 & 24 \\
\hline 8 & 0 & 4 & 5 & 0 & 13 & 22 \\
\hline 9 & 3 & 0 & 7 & 13 & 0 & 23 \\
\hline 10 & 0 & 5 & 8 & 0 & 10 & 23 \\
\hline
\end{tabular}




\section{CHRONIC DEFINITIVE SURVIVAL AND REPRODUCTION TEST \\ Statistical Analyses}

Client:

WSRC

Sample Identification:

Test Date:
A-01

September 10, 2002

\section{Test for Normality}

Test Used: Kolmogorov D

NA

$\begin{array}{ll}D= & N A \\ \text { critical } & N A\end{array}$

Test for Homogeneity of Variance

Teșt Used: Bartlett's Test

$B=N A$

critical NA

NA

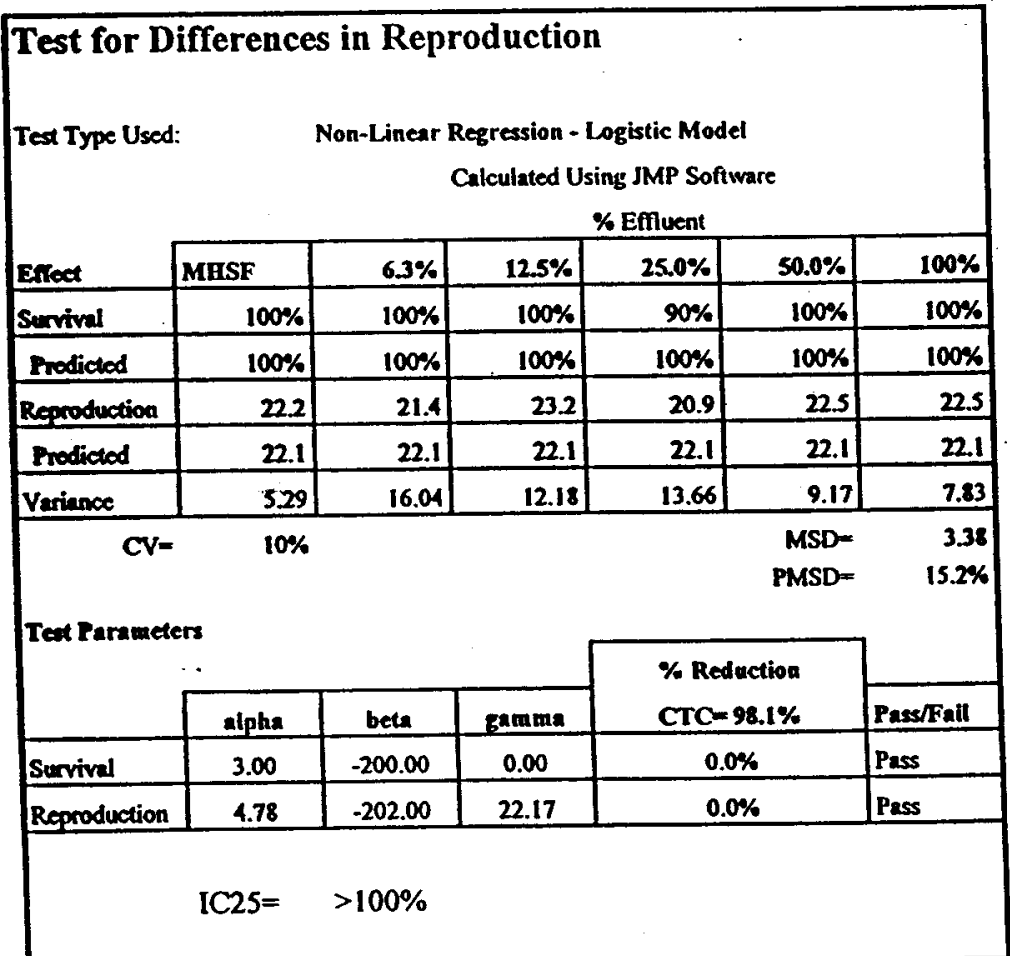

\section{Reproduction vs. Effluent Conc.}

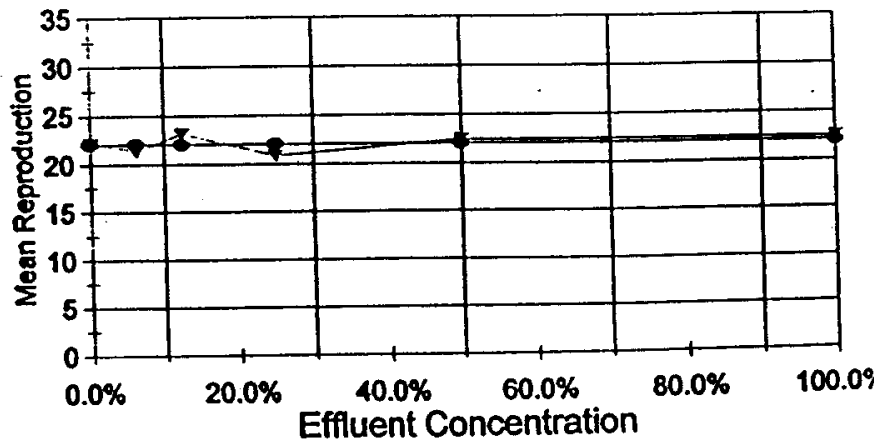

Actual Reproduction

Summary

The reductions in survival and reproduction at the CTC are $<5 \%$ - Pass 


\section{Day Chronic Definitive Survival and Reproduction Bioassay \\ Method: EPA/600/4-91/002}

Test Organism:

Daphnia ambigua

Facility: WSRC

Sample ID: A-11

County

ETT\#: 20570

Sample ID: September 10, 2002 


\section{CHRONIC DEFINITIVE SURVIVAL AND REPRODUCTION TEST \\ Survival and Reproduction Results}

Client:

Sample Identification:

Test Date:

\begin{tabular}{|c|c|c|c|c|c|c|}
\hline \multicolumn{3}{|c|}{ Reproduction at } & \multirow{2}{*}{$\frac{\text { MHSF }}{\text { Day } 6}$} & \multicolumn{3}{|l|}{ Control } \\
\hline Rep. & Day 3/4 & Day 5 & & Day 7 & Day 8 & Total \\
\hline 1 & 0 & 5 & 8 & 0 & 10 & 23 \\
\hline 2 & 0 & 3 & 7 & 0 & 12 & 22 \\
\hline 3 & 0 & 5 & 5 & 0 & 11 & 21 \\
\hline 4 & 0 & 4 & 5 & 0 & 13 & 22 \\
\hline 5 & 4 & 0 & 5 & 9 & 0 & 18 \\
\hline 6 & 0 & 5 & 0 & 9 & 13 & 27 \\
\hline 7 & 5 & 0 & 6 & 11 & 0 & 22 \\
\hline 8 & 0 & 4 & 0 & 7 & 10 & 21 \\
\hline 9 & 0 & 4 & 0 & 7 & 11 & 22 \\
\hline 10 & 0 & 4 & 7 & 0 & 13 & 24 \\
\hline \multicolumn{3}{|c|}{ Reproduction at } & \multicolumn{2}{|c|}{$12.5 \%$} & \multicolumn{2}{|c|}{ Concentration } \\
\hline Rep. & Day 3/4 & Day 5 & Day 6 & Day 7 & Day 8 & Total \\
\hline 1 & 4 & 0 & 8 & 10 & 0 & 22 \\
\hline 2 & 4 & 0 & 7 & 13 & 0 & 24 \\
\hline 3 & 0 & 4 & 5 & 0 & 12 & 21 \\
\hline 4 & 0 & 5 & 5 & 0 & 13 & 23 \\
\hline 5 & 3 & 1 & 4 & 11 & 0 & 19 \\
\hline 6 & 0 & 0 & 4 & 8 & 0 & 12 \\
\hline 7 & 4 & 0 & $7^{-}$ & 12 & 0 & 23 \\
\hline 8 & 0 & 4 & 5 & 0 & 9 & 18 \\
\hline 9 & 0 & 0 & 4 & 8 & 0 & 12 \\
\hline 10 & 0 & 1 & 7 & 0 & 10 & 18 \\
\hline
\end{tabular}

\begin{tabular}{|c|c|c|c|c|c|c|}
\hline Reproduction at & \multicolumn{4}{c|}{$50 \%$} & Concentration \\
\hline Rep. & Day 3/4 & Day 5 & Day 6 & Day 7 & Day & Total \\
\hline 1 & 3 & 0 & 8 & 12 & 0 & 23 \\
\hline 2 & 0 & 0 & 1 & 0 & 0 & 1 \\
\hline 3 & 4 & 0 & 7 & 13 & 0 & 24 \\
\hline 4 & 0 & 0 & 7 & 0 & 10 & 17 \\
\hline 5 & 3 & 0 & 5 & 12 & 0 & 20 \\
\hline 6 & 4 & 0 & 9 & 9 & 0 & 22 \\
\hline 7 & 5 & 0 & 7 & 14 & 0 & 26 \\
\hline 8 & 0 & 4 & 7 & 0 & 13 & 24 \\
\hline 9 & 0 & 6 & 6 & 0 & 10 & 22 \\
\hline 10 & 0 & 4 & 7 & 0 & 10 & 21 \\
\hline
\end{tabular}

County:

NPDES\#: $\quad$ SC

ETT \#:

20570

\begin{tabular}{|c|c|c|c|c|c|c|}
\hline \multicolumn{6}{|c|}{ Reproduction at } & \multicolumn{6}{c|}{$6.25 \%$} & \multicolumn{3}{c|}{ Concentration } \\
\hline Rep. & Day 3/4 & Day 5 & Day 6 & Day 7 & Day 8 & Total \\
\hline 1 & 4 & 0 & 8 & 14 & 0 & 26 \\
\hline 2 & 0 & 4 & 7 & 0 & 9 & 20 \\
\hline 3 & 5 & 0 & 10 & 12 & 0 & 27 \\
\hline 4 & 0 & 4 & 8 & 0 & 11 & 23 \\
\hline 5 & 0 & 1 & 5 & 9 & 0 & 15 \\
\hline 6 & 4 & 0 & 9 & 13 & 0 & 26 \\
\hline 7 & 3 & 0 & 9 & 13 & 0 & 25 \\
\hline 8 & 0 & 3 & 7 & 0 & 9 & 19 \\
\hline 9 & 0 & 4 & 1 & 10 & 12 & 27 \\
\hline 10 & 0 & 4 & 5 & 0 & 10 & 19 \\
\hline
\end{tabular}

\begin{tabular}{|c|c|c|c|c|c|c|}
\hline \multicolumn{2}{|c|}{ Reproduction at } & \multicolumn{2}{c|}{$25 \%$} & \multicolumn{3}{c|}{ Concentration } \\
\hline Rep. & Day 3/4 & Day 5 & Day 6 & Day 7 & Day 8 & Toul \\
\hline 1 & 3 & 0 & 8 & 13 & 0 & 24 \\
\hline 2 & 4 & 0 & 2 & 9 & 1 & 16 \\
\hline 3 & 4 & 0 & 9 & 12 & 0 & 25 \\
\hline 4 & 0 & 5 & 7 & 0 & 12 & 24 \\
\hline 5 & 4 & 0 & 7 & 14 & 0 & 25 \\
\hline 6 & 0 & 6 & 10 & 0 & 13 & 29 \\
\hline 7 & 3 & 0 & 7 & 11 & 0 & 21 \\
\hline 8 & 0 & 3 & 5 & 0 & 8 & 16 \\
\hline 9 & 4 & 0 & 8 & 13 & 0 & 25 \\
\hline 10 & 0 & 5 & 8 & 0 & 10 & 23 \\
\hline
\end{tabular}

\begin{tabular}{|c|c|c|c|c|c|c|}
\hline Reproduction at & \multicolumn{4}{c}{$100 \%$} & \multicolumn{2}{c}{ Concentration } \\
\hline Rep. & Day 3/4 & Day 5 & Day 6 & Day 7 & Day 8 & Total \\
\hline 1 & 3 & 0 & 7 & 13 & 0 & 23 \\
\hline 2 & 1 & $X$ & $X$ & $X$ & $X$ & 1 \\
\hline 3 & 4 & 0 & 8 & 12 & 0 & 24 \\
\hline 4 & 0 & 4 & 7 & 0 & 11 & 22 \\
\hline 5 & 0 & 5 & 8 & 0 & 10 & 23 \\
\hline 6 & 3 & 0 & 8 & 10 & 0 & 21 \\
\hline 7 & 2 & 0 & 7 & 13 & 0 & 22 \\
\hline 8 & 0 & 3 & 5 & 0 & 8 & 16 \\
\hline 9 & 0 & 5 & 8 & 0 & 12 & 25 \\
\hline 10 & 0 & 4 & 7 & 0 & 13 & 24 \\
\hline
\end{tabular}




\section{CHRONIC DEFINITIVE SURVIVAL AND REPRODUCTION TEST \\ Statistical Analyses}

Client:

Sample Identification:

Test Date:
WSRC

A-11

September 10, 2002

\section{Test for Normality}

Test Used: Kolmogorov D

$\begin{array}{ll}\mathrm{D}= & \mathrm{NA} \\ \text { critical } & \mathrm{NA}\end{array}$

NA
Test for Homogeneity of Variance

Test Used: Bartlett's Test

$\mathrm{B}=\mathrm{NA}$

critical NA

\section{NA}

Reproduction vs. Effluent Conc.

Actual Reproduction

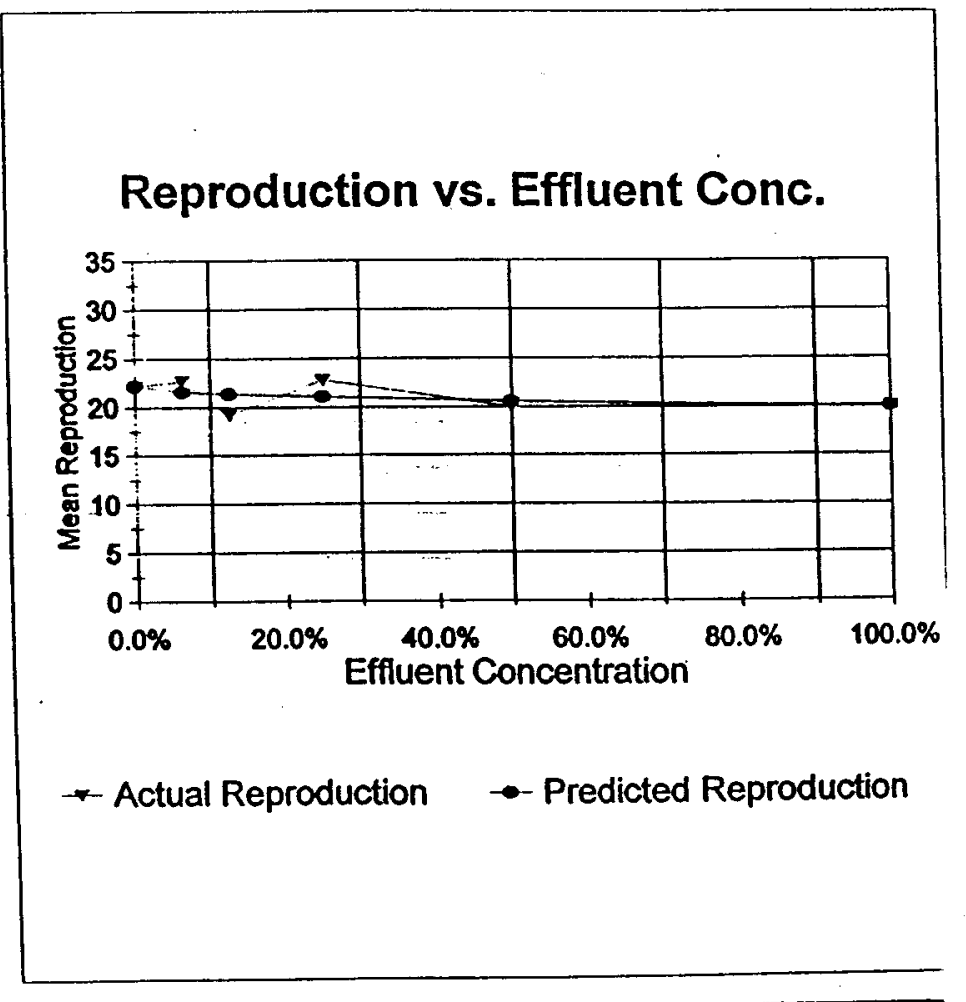

Summary 


\section{Day Chronic Definitive Survival and Reproduction Bioassay Method: EPA/600/4-91/002}

$$
\begin{aligned}
& \therefore \\
& \text {.... } \\
& =
\end{aligned}
$$

Test Organism:

Daphnia ambigua

Facility: WSRC

Sample ID: G-10

$$
\text { County }
$$

ETT\#: 20571

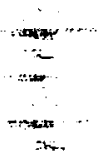

Sample ID: September 10, 2002 


\section{CHRONIC DEFINITIVE SURVIVAL AND REPRODUCTION TEST Survival and Reproduction Results}

Client:

Sample Identification:

Test Date:

WSRC

September 10, 2002
County:

NPDES\#: $\quad$ SC

ETT \#:
20571

\begin{tabular}{|c|c|c|c|c|c|c|}
\hline Reproduction at & \multicolumn{7}{c|}{ MHSF } & Control \\
\hline Rep. & Day 3/4 & Day 5 & Day 6 & Day 7 & Day 8 & Total \\
\hline 1 & 0 & 5 & 9 & 0 & 12 & 26 \\
\hline 2 & 0 & 4 & 9 & 0 & 12 & 25 \\
\hline 3 & 3 & 0 & 5 & 9 & 0 & 17 \\
\hline 4 & 3 & 0 & 6 & 0 & 11 & 20 \\
\hline 5 & 0 & 0 & 7 & 0 & 12 & 19 \\
\hline 6 & 0 & 0 & 6 & 0 & 13 & 19 \\
\hline 7 & 0 & 5 & 8 & 0 & 12 & 25 \\
\hline 8 & 0 & 6 & 6 & 0 & 13 & 25 \\
\hline 9 & 5 & 0 & 6 & 9 & 0 & 20 \\
\hline 10 & 4 & 0 & 8 & 12 & 0 & 24 \\
\hline
\end{tabular}

\begin{tabular}{|c|c|c|c|c|c|c|}
\hline \multicolumn{2}{|c|}{ Reproduction at } & \multicolumn{2}{c|}{$6.25 \%$} & \multicolumn{1}{c|}{ Concentration } \\
\hline Rep. & Day 3/4 & Day 5 & Day 6 & Day 7 & Day 8 & Total \\
\hline 1 & 0 & 5 & 6 & 0 & 13 & 24 \\
\hline 2 & 0 & 6 & 7 & 0 & 10 & 23 \\
\hline 3 & 5 & 0 & 9 & 12 & 0 & 26 \\
\hline 4 & 0 & 4 & $X$ & $X$ & $X$ & 4 \\
\hline 5 & 6 & 0 & 8 & 10 & 0 & 24 \\
\hline 6 & 0 & 6 & 8 & 0 & 13 & 27 \\
\hline 7 & 0 & 5 & 7 & 0 & 0 & 12 \\
\hline 8 & 4 & 0 & 8 & 9 & 0 & 21 \\
\hline 9 & 0 & 5 & 6 & 0 & 11 & 22 \\
\hline 10 & 3 & 0 & 9 & 13 & 0 & 25 \\
\hline
\end{tabular}

\begin{tabular}{|c|c|c|c|c|c|c|}
\hline \multicolumn{2}{|c|}{ Reproduction at } & \multicolumn{3}{c|}{$12.5 \%$} & \multicolumn{3}{c|}{ Concentration } \\
\hline Rep. & Day 3/4 & Day 5 & Day 6 & Day 7 & Day 8 & Total \\
\hline 1 & 0 & 5 & 11 & 0 & 13 & 29 \\
\hline 2 & 0 & 5 & 7 & 0 & 13 & 25 \\
\hline 3 & 4 & 0 & 8 & 12 & 0 & 24 \\
\hline 4 & 0 & 6 & 6 & 12 & 0 & 24 \\
\hline 5 & 4 & 0 & 9 & 12 & 0 & 25 \\
\hline 6 & 0 & 5 & 10 & 0 & 12 & 27 \\
\hline 7 & 0 & 5 & 7 & 0 & 10 & 22 \\
\hline 8 & 0 & 4 & 6 & 0 & 13 & 23 \\
\hline 9 & 4 & 0 & 7 & 10 & 0 & 21 \\
\hline 10 & 5 & 0 & 9 & 13 & 0 & 27 \\
\hline
\end{tabular}

\begin{tabular}{|c|c|c|c|c|c|c|}
\hline \multicolumn{2}{|c|}{ Reproduction at } & \multicolumn{2}{c|}{$25 \%$} & \multicolumn{3}{c|}{ Concentration } \\
\hline Rep. & Day 3/4 & Day 5 & Day 6 & Day 7 & Day 8 & Total \\
\hline 1 & 0 & 4 & 7 & 0 & 11 & 22 \\
\hline 2 & 0 & 5 & 8 & 10 & 0 & 23 \\
\hline 3 & 0 & 0 & 7 & 7 & 2 & 16 \\
\hline 4 & 0 & 5 & 7 & 0 & 13 & 25 \\
\hline 5 & 0 & 5 & 6 & 13 & 0 & 24 \\
\hline 6 & 0 & 6 & 8 & 0 & 12 & 26 \\
\hline 7 & 0 & 4 & 8 & 0 & 13 & 25 \\
\hline 8 & 0 & 3 & 7 & 0 & 13 & 23 \\
\hline 9 & 3 & 0 & 6 & 10 & 0 & 19 \\
\hline 10 & 3 & 0 & 7 & 8 & 0 & 18 \\
\hline
\end{tabular}

\begin{tabular}{|c|c|c|c|c|c|c|}
\hline \multicolumn{1}{|c|}{ Reproduction at } & \multicolumn{3}{c|}{$50 \%$} & \multicolumn{3}{c|}{ Concentration } \\
\hline Rep. & Day 3/4 & Day 5 & Day 6 & Day 7 & Day 8 & Todal \\
\hline 1 & 4 & 0 & 7 & 14 & 0 & 25 \\
\hline 2 & 0 & 6 & 8 & 0 & 13 & 27 \\
\hline 3 & 6 & 0 & 4 & 13 & 0 & 23 \\
\hline 4 & 3 & 0 & 6 & 12 & 0 & 21 \\
\hline 5 & 0 & 5 & 7 & 13 & 0 & 25 \\
\hline 6 & 0 & 4 & 9 & 0 & 13 & 26 \\
\hline 7 & 0 & 5 & 8 & 0 & 9 & 22 \\
\hline 8 & 0 & 5 & 7 & 13 & 0 & 25 \\
\hline 9 & 0 & 5 & 5 & 0 & 9 & 19 \\
\hline 10 & 3 & 0 & 7 & 14 & 0 & 24 \\
\hline
\end{tabular}

\begin{tabular}{|c|c|c|c|c|c|c}
\hline Reproduction at & \multicolumn{2}{c}{ 100\% } & \multicolumn{3}{c}{ Concentration } \\
\hline Rep. & Day 3/4 & Day 5 & Day 6 & Day 7 & Day 8 & Total \\
\hline 1 & 0 & 4 & 9 & 0 & 13 & 26 \\
\hline 2 & 0 & 4 & 7 & 0 & 10 & 21 \\
\hline 3 & 4 & 0 & 8 & 12 & 0 & 24 \\
\hline 4 & 3 & 0 & 4 & 14 & 0 & 21 \\
\hline 5 & 0 & 5 & 7 & 0 & 8 & 20 \\
\hline 6 & 0 & 6 & 8 & 0 & 11 & 25 \\
\hline 7 & 0 & 4 & 3 & $X$ & $X$ & 7 \\
\hline 8 & 0 & 3 & 5 & 0 & 11 & 19 \\
\hline 9 & 3 & 0 & 3 & 12 & 0 & 18 \\
\hline 10 & 4 & 0 & 5 & 14 & 0 & 23 \\
\hline
\end{tabular}




\section{CHRONIC DEFINITIVE SURVIVAL AND REPRODUCTION TEST \\ Statistical Analyses}

Client:

Sample Identification:

Test Date:
WSRC

G-10

September 10, 2002
Test Used: Kolmogorov D

$\begin{array}{ll}D= & \text { NA } \\ \text { critical } & \text { NA }\end{array}$

NA

\section{Test for Normality}

\begin{tabular}{|c|} 
Test for Homogeneity of Variance \\
Test Used: Bartlett's Test $\quad \begin{array}{r}\text { B }=\text { NA } \\
\text { critical }\end{array}$ \\
NA
\end{tabular}

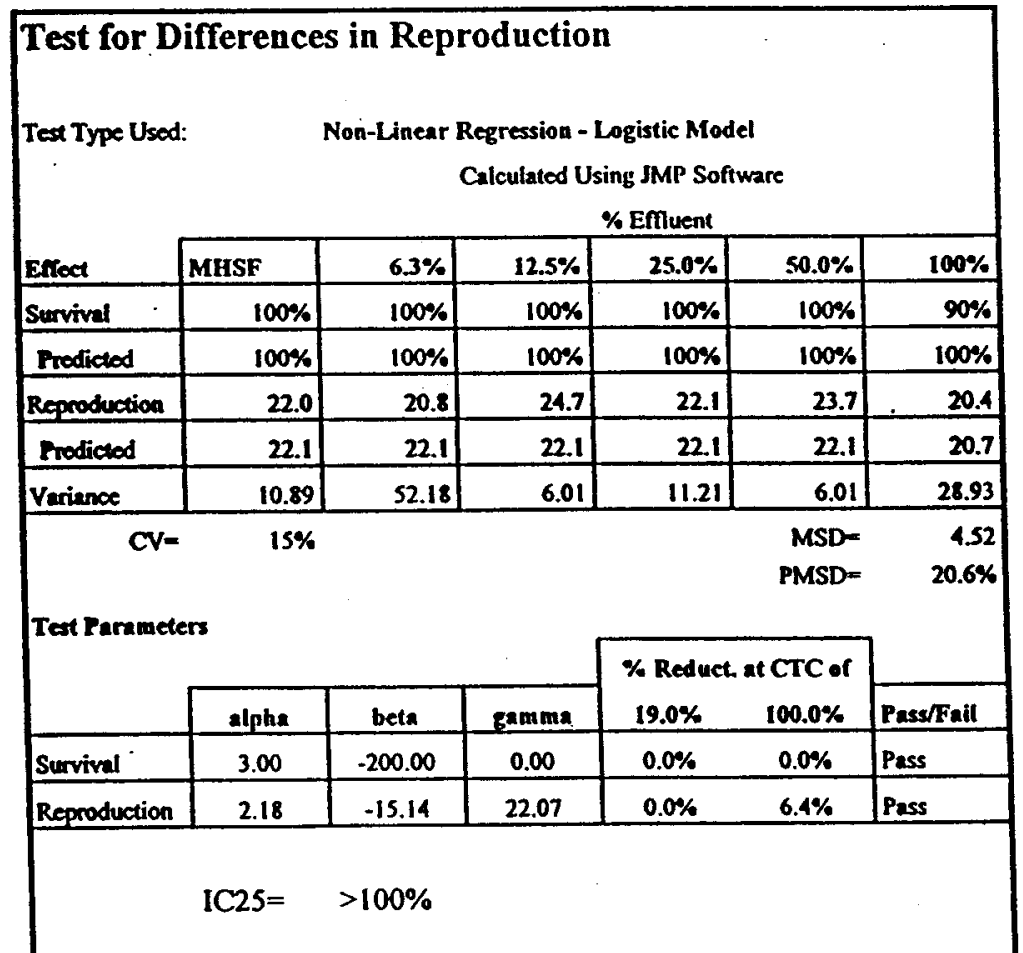

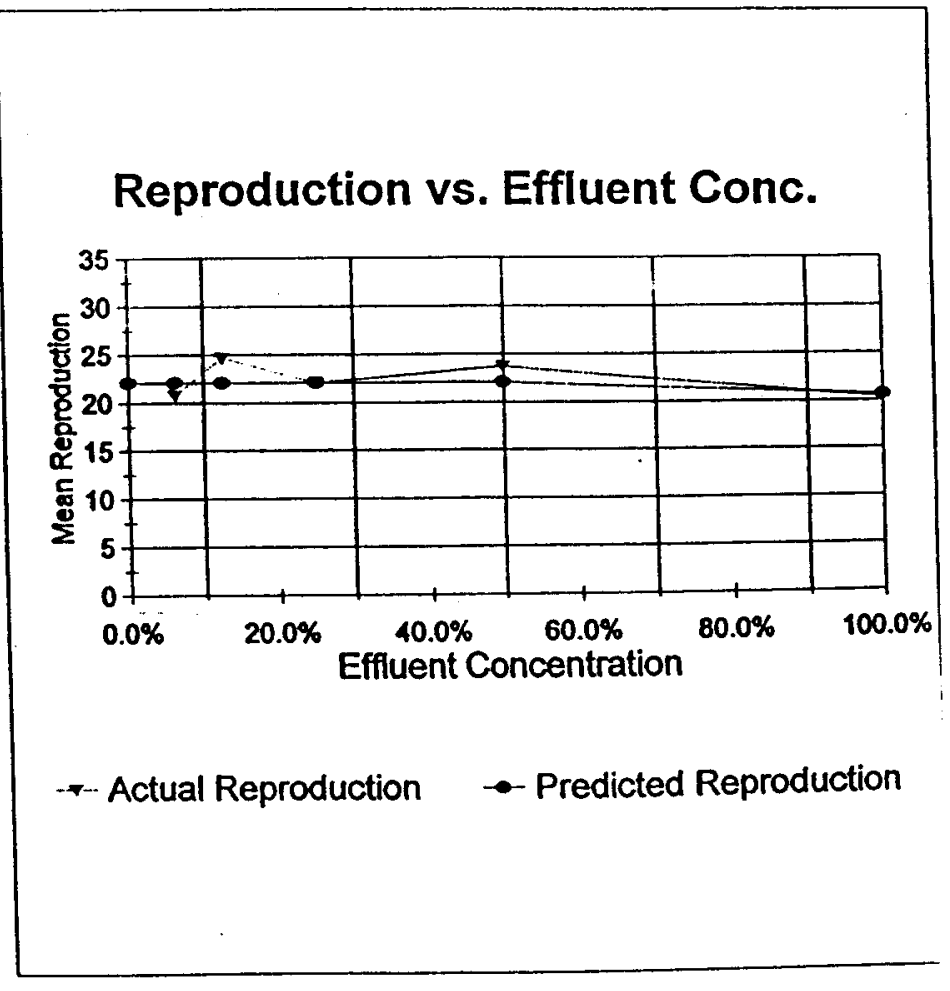

Summary

The reductions in survival and reproduction at the CTC are $<25 \%$ - Pass 


\section{CHRONIC DEFINITIVE SURVIVAL AND REPRODUCTION TEST \\ Survival and Reproduction Results}

Client:

Sample Identification:

Test Date:

WSRC

September 10, 2002

\begin{tabular}{|c|c|c|c|c|c|c|}
\hline \multicolumn{2}{|c|}{ Reproduction at } & \multicolumn{7}{c|}{ MHSF } & Control \\
\hline Rep. & Day 3/4 & Day 5 & Day 6 & Day 7 & Day 8 & Total \\
\hline 1 & 0 & 5 & 9 & 0 & 12 & 26 \\
\hline 2 & 0 & 4 & 9 & 0 & 12 & 25 \\
\hline 3 & 3 & 0 & 5 & 9 & 0 & 17 \\
\hline 4 & 3 & 0 & 6 & 0 & 11 & 20 \\
\hline 5 & 0 & 0 & 7 & 0 & 12 & 19 \\
\hline 6 & 0 & 0 & 6 & 0 & 13 & 19 \\
\hline 7 & 0 & 5 & 8 & 0 & 12 & 25 \\
\hline 8 & 0 & 6 & 6 & 0 & 13 & 25 \\
\hline 9 & 5 & 0 & 6 & 9 & 0 & 20 \\
\hline 10 & 4 & 0 & 8 & 12 & 0 & 24 \\
\hline
\end{tabular}

\begin{tabular}{|c|c|c|c|c|c|c|}
\hline \multicolumn{2}{|c|}{ Reproduction at } & \multicolumn{4}{c|}{$12.5 \%$} & Concentration \\
\hline Rep. & Day 3/4 & Day 5 & Day 6 & Day 7 & Day 8 & Total \\
\hline 1 & 0 & 5 & 11 & 0 & 13 & 29 \\
\hline 2 & 0 & 5 & 7 & 0 & 13 & 25 \\
\hline 3 & 4 & 0 & 8 & 12 & 0 & 24 \\
\hline 4 & 0 & 6 & 6 & 12 & 0 & 24 \\
\hline 5 & 4 & 0 & 9 & 12 & 0 & 25 \\
\hline 6 & 0 & 5 & 10 & 0 & 12 & 27 \\
\hline 7 & 0 & 5 & 7 & 0 & 10 & 22 \\
\hline 8 & 0 & 4 & 6 & 0 & 13 & 23 \\
\hline 9 & 4 & 0 & 7 & 10 & 0 & 21 \\
\hline 10 & 5 & 0 & 9 & 13 & 0 & 27 \\
\hline
\end{tabular}

\begin{tabular}{|c|c|c|c|c|c|c|}
\hline \multicolumn{2}{|c|}{ Reproduction at } & \multicolumn{3}{c|}{$50 \%$} & \multicolumn{3}{c|}{ Concentration } \\
\hline Rep. & Day 3/4 & Day 5 & Day 6 & Day 7 & Day 8 & Total \\
\hline 1 & 4 & 0 & 7 & 14 & 0 & 25 \\
\hline 2 & 0 & 6 & 8 & 0 & 13 & 27 \\
\hline 3 & 6 & 0 & 4 & 13 & 0 & 23 \\
\hline 4 & 3 & 0 & 6 & 12 & 0 & 21 \\
\hline 5 & 0 & 5 & 7 & 13 & 0 & 25 \\
\hline 6 & 0 & 4 & 9 & 0 & 13 & 26 \\
\hline 7 & 0 & 5 & 8 & 0 & 9 & 22 \\
\hline 8 & 0 & 5 & 7 & 13 & 0 & 25 \\
\hline 9 & 0 & 5 & 5 & 0 & 9 & 19 \\
\hline 10 & 3 & 0 & 7 & 14 & 0 & 24 \\
\hline
\end{tabular}

County:

NPDESH:

ETT \#:

$\mathrm{SC}$

20571

\begin{tabular}{|c|c|c|c|c|c|c|}
\hline \multicolumn{2}{|c|}{ Reproduction at } & \multicolumn{2}{c|}{$6.25 \%$} & \multicolumn{2}{c|}{ Concentration } \\
\hline Rep. & Day 3/4 & Day 5 & Day 6 & Day 7 & Day 8 & Total \\
\hline 1 & 0 & 5 & 6 & 0 & 13 & 24 \\
\hline 2 & 0 & 6 & 7 & 0 & 10 & 23 \\
\hline 3 & 5 & 0 & 9 & 12 & 0 & 26 \\
\hline 4 & 0 & 4 & $X$ & $X$ & $X$ & 4 \\
\hline 5 & 6 & 0 & 8 & 10 & 0 & 24 \\
\hline 6 & 0 & 6 & 8 & 0 & 13 & 27 \\
\hline 7 & 0 & 5 & 7 & 0 & 0 & 12 \\
\hline 8 & 4 & 0 & 8 & 9 & 0 & 21 \\
\hline 9 & 0 & 5 & 6 & 0 & 11 & 22 \\
\hline 10 & 3 & 0 & 9 & 13 & 0 & 25 \\
\hline
\end{tabular}

\begin{tabular}{|c|c|c|c|c|c|c|}
\hline \multicolumn{2}{|c|}{ Reproduction at } & \multicolumn{3}{|c|}{$25 \%$} & \multicolumn{2}{c|}{ Concentration } \\
\hline Rep. & Day 3/4 & Day 5 & Day 6 & Day 7 & Day 8 & Total \\
\hline 1 & 0 & 4 & 7 & 0 & 11 & 22 \\
\hline 2 & 0 & 5 & 8 & 10 & 0 & 23 \\
\hline 3 & 0 & 0 & 7 & 7 & 2 & 16 \\
\hline 4 & 0 & 5 & 7 & 0 & 13 & 25 \\
\hline 5 & 0 & 5 & 6 & 13 & 0 & 24 \\
\hline 6 & 0 & 6 & 8 & 0 & 12 & 26 \\
\hline 7 & 0 & 4 & 8 & 0 & 13 & 25 \\
\hline 8 & 0 & 3 & 7 & 0 & 13 & 23 \\
\hline 9 & 3 & 0 & 6 & 10 & 0 & 19 \\
\hline 10 & 3 & 0 & 7 & 8 & 0 & 18 \\
\hline
\end{tabular}

\begin{tabular}{|c|c|c|c|c|c|c}
\hline \multicolumn{2}{|c|}{ Reproduction at } & \multicolumn{3}{c}{$100 \%$} & \multicolumn{3}{c}{ Concentration } \\
\hline Rep. & Day 3/4 & Day 5 & Day 6 & Day 7 & Day 8 & Tokal \\
\hline 1 & 0 & 4 & 9 & 0 & 13 & 26 \\
\hline 2 & 0 & 4 & 7 & 0 & 10 & 21 \\
\hline 3 & 4 & 0 & 8 & 12 & 0 & 24 \\
\hline 4 & 3 & 0 & 4 & 14 & 0 & 21 \\
\hline 5 & 0 & 5 & 7 & 0 & 8 & 20 \\
\hline 6 & 0 & 6 & 8 & 0 & 11 & 25 \\
\hline 7 & 0 & 4 & 3 & $X$ & $X$ & 7 \\
\hline 8 & 0 & 3 & 5 & 0 & 11 & 19 \\
\hline 9 & 3 & 0 & 3 & 12 & 0 & 18 \\
\hline 10 & 4 & 0 & 5 & 14 & 0 & 23 \\
\hline
\end{tabular}




\section{CHRONIC DEFINITIVE SURVIVAL AND REPRODUCTION TEST \\ Water Chemistry}

Client: $\quad$ WSRC

Sample Identification: A-01

Test Date: $\quad$ February 26, 2002

\section{pH (After Renewal)}

\begin{tabular}{|l|r|r|r|r|r|r|}
\hline & \multicolumn{7}{|c|}{ Test Concentration } \\
\hline & control & $6.25 \%$ & $12.5 \%$ & $25 \%$ & $50 \%$ & $100 \%$ \\
\hline Initial & 7.75 & 7.46 & 7.12 & 7.07 & 7.04 & 7.00 \\
\hline Day 1 & 8.20 & 8.06 & 7.80 & 7.59 & 7.43 & 7.09 \\
\hline Day 2 & 7.11 & 6.93 & 6.85 & 7.93 & 7.07 & 7.24 \\
\hline Day 3 & 7.01 & 7.15 & 7.02 & 6.98 & 7.04 & 7.13 \\
\hline Day 4 & 7.02 & 7.23 & 7.19 & 7.11 & 7.05 & 6.98 \\
\hline Day 5 & 7.06 & 7.11 & 7.28 & 7.25 & 7.07 & 7.01 \\
\hline Day 6 & 7.04 & 6.95 & 6.85 & 6.94 & 6.96 & 7.11 \\
\hline
\end{tabular}

Dissolved Oxygen (After Renewal)

\begin{tabular}{|l|r|r|r|r|r|r|}
\hline & \multicolumn{7}{|c|}{ Test Concentration } \\
\hline Initial & 8.4 & 8.2 & 8.2 & 8.5 & 8.3 & 8.4 \\
\hline Day 1 & 8.4 & 8.2 & 8.2 & 8.3 & 8.3 & 8.3 \\
\hline Day 2 & 8.4 & 8.3 & 8.3 & 8.4 & 8.4 & 8.4 \\
\hline Day 3 & 8.4 & 8.2 & 8.2 & 8.3 & 8.4 & 8.4 \\
\hline Day 4 & 8.4 & 8.2 & 8.2 & 8.4 & 8.4 & 8.4 \\
\hline Day 5 & 8.3 & 8.4 & 8.3 & 8.3 & 8.4 & 8.4 \\
\hline Day 6 & 8.4 & 8.3 & 8.3 & 8.3 & 8.4 & 8.4 \\
\hline
\end{tabular}

pH (Before Renewal)

\begin{tabular}{|l|r|r|r|r|r|r|}
\hline & control & $6.25 \%$ & $12.5 \%$ & $25 \%$ & $50 \%$ & $100 \%$ \\
\hline Day 1 & 8.25 & 8.09 & 7.92 & 7.83 & 7.86 & 7.66 \\
\hline Day 2 & 7.36 & 7.34 & 7.22 & 7.18 & 7.31 & 7.64 \\
\hline Day 3 & 7.34 & 7.26 & 7.23 & 7.24 & 7.30 & 7.43 \\
\hline Day 4 & 7.11 & 7.33 & 7.31 & 7.12 & 7.22 & 7.45 \\
\hline Day 5 & 7.09 & 7.18 & 7.26 & 7.24 & 7.36 & 7.38 \\
\hline Day 6 & 6.95 & 6.88 & 6.95 & 6.87 & 6.84 & 7.07 \\
\hline Final & 7.63 & 7.97 & & 7.38 & 7.43 & 7.47 \\
\hline
\end{tabular}

Dissolved Oxygen (Before Renewal)

\begin{tabular}{|l|r|r|r|r|r|r|}
\hline \multicolumn{7}{|c|}{ Test Concentration } \\
\hline & control & $6.25 \%$ & $12.5 \%$ & $25 \%$ & $50 \%$ & $100 \%$ \\
\hline Day 1 & 8.3 & 8.3 & 8.3 & 8.4 & 8.4 & 8.4 \\
\hline Day 2 & 8.5 & 8.4 & 8.4 & 8.3 & 8.4 & 8.4 \\
\hline Day 3 & 8.5 & 8.4 & 8.4 & 8.4 & 8.5 & 8.5 \\
\hline Day 4 & 8.5 & 8.4 & 8.5 & 8.5 & 8.5 & 8.5 \\
\hline Day 5 & 8.4 & 8.4 & 8.4 & 8.3 & 8.3 & 8.3 \\
\hline Day 6 & 8.3 & 8.4 & 8.4 & 8.4 & 8.4 & 8.4 \\
\hline Final & 8.4 & 8.4 & & 8.3 & 8.4 & 8.4 \\
\hline
\end{tabular}

Temperature

Temperature
\begin{tabular}{|l|r|}
\hline \multicolumn{1}{|c|}{ Incubator ${ }^{\circ} \mathrm{C}$} \\
\hline Initial & 25.5 \\
\hline Day 1 & 25.2 \\
\hline Day 2 & 25.2 \\
\hline Day 3 & 25.4 \\
\hline Day 4 & 25.4 \\
\hline Day 5 & 25.6 \\
\hline Day 6 & 25.4 \\
\hline Final & 25.0 \\
\hline
\end{tabular}

